\title{
Candidate Wind Turbine Generator Site Annual Data Summary for January 1979 Through December 1979
}
W. F. Sandusky
D. S. Renné

March 1981

Prepared for the U.S. Department of Energy under Contract DE-AC06-76RLO 1830

Pacific Northwest Laboratory

Operated for the U.S. Department of Energy by Battelle Memorial Institute 


\title{
NOTICE
}

This report was prepared as an account of work sponsored by the United States Government. Neither the United States nor the Department of Energy, nor any of their employees, nor any of their contractors, subcontractors, or their employees. makes any warranty, express or implied, or assumes any legal liability or responsibility for the accuracy, completeness or usefulness of any information, apparatus, product or process disclosed, or represents that its use would not infringe privately owned rights.

The views, opinions and conclusions contained in this report are those of the contractor and do not necessarily represent those of the United States Government or the United States Department of Energy.

\author{
PACIFIC NORTHWEST LABORATORY \\ operated by \\ BATTELLE \\ for the \\ UNITED STATES DEPARTMENT OF ENERGY \\ Under Contract DE-AC06-76RLO 1830
}

\author{
Printed in the United States of America \\ Available from \\ National Technical Information Service \\ United States Department of Commerce \\ 5285 Port Royal Road \\ Springfield, Virginia 22151
}

Price: Printed Copy

$\approx$ Microfiche $\$ 3.00$

NTIS

-Pages Selling Price

001-025 \$4.00

026-050 \$4.50

051-075 \$5.25

076-100 \$6.00

$101-125 \quad \$ 6.50$

126-150 \$7.25

$151-175 \quad \$ 8.00$

$176.200 \quad \$ 9.00$

201-225 \$9.25

226-250 \$9.50

251-275 $\$ 10.75$

$276-300 \quad \$ 11.00$ 


\section{5}

CANDIDATE WIND TURBINE GENERATOR SITE

ANNUAL DATA SUMMARY

FOR

JANUARY 1979 THROUGH DECEMBER 1979

by

W. F. Sandusky

D. S. Renne'

March 1981

Prepared for the

U.S. Department of Energy

Under Contract DE-AC06-76RL0 1830

Pacific Northwest Laboratory

Richland, Vashington 99352 


\section{SUMMARY}

Summarized hourly meteorological data for fifteen candidate and wind turbine - generator sites are presented in this report. These data are collected for the - Department of Energy for the purpose of evaluating the wind energy potential at these sites and are used to assist in selection of potential sites for installa-

- tion and testing of large wind turbines in electric utility systems. For each site, data are given in eight tables and one figure. Use of information from these tables, with information about specific wind turbines, should allow the user to estimate the potential for wind energy production at each site. 
CONTENTS

SUMMARY

INTRODUCTION

SUMMARY TABLES

AMARILLO, TEXAS

BLOCK ISLAND, RHODE ISLAND

BOARDMAN, OREGON

BOONE, NORTH CAROLINA

CLAYTON, NEW MEXICO .

COLD BAY, ALASKA .

CULEBRA, PUERTO RICO

HOLYOKE, MASSACHUSETTS .

HURON, SOUTH DAKOTA .

KINGSLEY DAM, NEBRASKA.

LUDINGTON, MICHIGAN .

MONTAUK POINT, NEW YORK

POINT ARENA, CALIFORNIA

RUSSELL, KANSAS

SAN GORGONIO PASS, CALIFORNIA 


\section{INTRODUCTION}

In the spring of 1976 the Department of Energy (then known as the Energy Research and Development Administration) selected 17 candidate sites, each proposed by interested electric service organizations, to receive meteorological measuring equipment for the purpose of evaluating the wind energy potential at these sites. The Department of Energy's Wind Energy Systems Division uses this meteorological information to assist in selection of potential sites for installation and field testing of large wind turbines in electric utility systems. Meteorological measurements at these sites began in late 1976 and early 1977, and in 1979 data collection programs were operable at 16 sites.

This report presents summarized hourly meteorological data based on 2-min data at 15 of the candidate and wind turbine generator installation sites listed in Table 1. The data cover the period from January, 1979, through December, 1979. The data were collected from sensors at two or three levels on a meteorological tower. Selection of the levels was determined by site characteristics (and in one case, tower height). The data are recorded digitally at the site on a data cassette recording system with an instantaneous sample of data recorded every two minutes.

The two sites for which data are not reported are Kaena Point, Hawaii, and Augspurger Mountain, Washington. The collection program and data analys is for the Kaena Point site was directed by the Department of Meteorology at the University of Hawaii. Data collection continued at the site until November, 1979. Pacific Northwest Laboratory received summarized data in a different format than reported herein and thus the Kaena Point data are not included. The collection program at Augspurger Mountain continued until the tower collapsed in January, 1978.

The heights of the sensors at each site are given in Table 2. In that table and in later summary tables, the upper sensor is noted by the letter "A", the mid-level is noted by the letter "B" and the lower level is noted by the letter "C." Note that only four sites (Block Island, Rhode Island; Boone, North Carolina; Clayton, New Mexico; and Boardman, Oregon) have mid-level sensors. 
TABLE 1. Site Identification and Location

\begin{tabular}{|c|c|c|c|c|c|}
\hline Site & $\begin{array}{c}\text { Identification } \\
\text { Code } \\
\end{array}$ & $\begin{array}{l}\text { Date Collection } \\
\text { Began } \\
\end{array}$ & Latitude & Longi tude & $\begin{array}{l}\text { Elevation } \\
\text { (meters) }\end{array}$ \\
\hline Amarillo, TX & $T X$ & $03 / 01 / 77$ & $35^{\circ} 17^{\prime} \mathrm{N}$ & $101^{\circ} 45^{\prime} \mathrm{W}$ & 1091 \\
\hline Block Island, RI & RI & $12 / 11 / 76$ & $41^{\circ} 10^{\prime} \mathrm{N}$ & $71^{\circ} 34^{\prime} \mathrm{W}$ & 14 \\
\hline Boardman, OR & OR & $01 / 01 / 77$ & $45^{\circ} 41^{\prime} \mathrm{N}$ & $119^{\circ} 50^{\prime} \mathrm{W}$ & 212 \\
\hline Boone, NC & NC & $12 / 14 / 76$ & $36^{\circ} 15^{\prime} \mathrm{N}$ & $81^{\circ} 40^{\prime} \mathrm{W}$ & 1347 \\
\hline Clayton, NM & NM & $05 / 01 / 77$ & $36^{\circ} 26^{\prime} \mathrm{N}$ & $103^{\circ} 12^{\prime} \mathrm{W}$ & 1536 \\
\hline Cold Bay, AK & $A K$ & $08 / 01 / 77$ & $55^{\circ} 12^{\prime} \mathrm{N}$ & $162^{\circ} 43^{\prime} \mathrm{W}$ & 29 \\
\hline Culebra, PR & PR & $03 / 01 / 77$ & $18^{\circ} 20^{\prime} \mathrm{N}$ & $65^{\circ} 18^{\prime} W$ & 80 \\
\hline Holyoke, MA & MA & $12 / 16 / 76$ & $42^{\circ} 15^{\prime} \mathrm{N}$ & $72^{\circ} 38^{\prime} \mathrm{W}$ & 372 \\
\hline Huron, SD & SD & $12 / 10 / 76$ & $44^{\circ} 24^{\prime} \mathrm{N}$ & $98^{\circ} 08^{\prime} W$ & 396 \\
\hline Kingsley, NB & NB & $12 / 05 / 76$ & $41^{\circ} 10^{\prime} \mathrm{N}$ & $101^{\circ} 39^{\prime} \mathrm{W}$ & 1024 \\
\hline Ludington, MI & MI & $04 / 01 / 77$ & $43^{\circ} 53^{\prime} \mathrm{N}$ & $86^{\circ} 26^{\prime} W$ & 213 \\
\hline Montauk, NY & NY & $12 / 30 / 76$ & $41^{\circ} 03^{1} \mathrm{~N}$ & $71^{\circ} 53^{\prime} \mathrm{W}$ & 2 \\
\hline Point Arena, CA & $C A$ & $01 / 07 / 77$ & $38^{\circ} 56^{\prime} \mathrm{N}$ & $123^{\circ} 43^{\prime} \mathrm{W}$ & 21 \\
\hline Russell, KS & KS & $12 / 02 / 76$ & $38^{\circ} 50^{\prime} \mathrm{N}$ & $98^{\circ} 51^{\prime} W$ & 564 \\
\hline San Gorgonio, CA & $S G$ & $12 / 03 / 76$ & $33^{\circ} 56^{\prime} \mathrm{N}$ & $116^{\circ} 34^{\prime} \mathrm{N}$ & 329 \\
\hline
\end{tabular}

TABLE 2. Sensor Heights

\begin{tabular}{|c|c|c|c|}
\hline Site Location & $\begin{array}{l}\text { C-Lower } \\
\text { Level(a) }\end{array}$ & $\begin{array}{l}\text { B-Middle } \\
\text { Level(a) } \\
\end{array}$ & $\begin{array}{l}\text { A-Upper } \\
\text { Level(a) }\end{array}$ \\
\hline Amarillo, TX & 9.1 & (b) & 45.7 \\
\hline Block Island, RI & 9.1 & 30.0 & 45.7 \\
\hline Boardman, OR & 9.1 & 39.6 & 70.1 \\
\hline Boone, NC & 13.2 & 45.7 & 76.2 \\
\hline Clayton, NM & 9.1 & 30.0 & 45.7 \\
\hline Cold Bay, AK & 9.1 & (b) & 21.8 \\
\hline Culebra, PR & 9.1 & (b) & 45.7 \\
\hline Holyoke, MA & 18.2 & (b) & 45.7 \\
\hline Huron, SD & 9.1 & (b) & 45.7 \\
\hline Kingsley Dam, NB & 9.1 & (b) & 45.7 \\
\hline Ludington, MI & 18.2 & (b) & 45.7 \\
\hline Montauk, NY & 18.2 & (b) & 45.7 \\
\hline Point Arena, $C A$ & 9.1 & (b) & 45.7 \\
\hline Russe11, KS & 9.1 & (b) & 45.7 \\
\hline San Gorgonio, CA & 9.1 & (b) & 45.7 \\
\hline
\end{tabular}

(a) Meters above ground level.

(b) No data collected. 
In some cases, circumstances dictated the height of the sensor levels. The height of the sensor levels at Boone was increased to accommodate the MOD-1 turbine installed at the site. This turbine has a larger blade diameter and taller tower than the MOD-OA. At the Boardman site the levels reported are based on an ongoing monitoring program for the construction and operation of a nearby coal plant. The upper-level sensor at the Cold Bay, Alaska, site is at $21.8 \mathrm{~m}$ since an existing FAA beacon tower is used to support the sensors. Four sites have the lower sensors at $18.2 \mathrm{~m}$ above ground level to measure wind data above trees or buildings near the sites.

Table 3 provides an all-site summary of data recovery rates and wind speed characteristics for the calendar year 1979. In that table, data under the column "S.D." represent standard deviation of wind speeds about the mean. Data under the column "MAX" represent maximum hourly average values during the reporting period.

Table 4 presents a summary of the mean annual wind speed obtained from each sensor level for the duration of this program to date. A 3-yr mean is also included.

The data for each site are presented in summary tables; the format of these tables is described in the next section. Information in the summary tables is provided on data recovery rates, maximum values observed, annual mean values, diurnal mean values, frequency distribution of wind speed, wind speed persistence, and power law exponent as a function of wind direction. The information from the summary tables combined with information about specific wind turbines should allow the user to estimate the potential for wind energy production at each site. 
TABLE 3. Summary of Hourly Data for the Period January 1979 Through December 1979

\begin{tabular}{|c|c|c|c|c|c|}
\hline $\begin{array}{l}\text { SITE } \\
\text { CODE }\end{array}$ & $\begin{array}{l}\text { SENSOR } \\
\text { LEVEL (M) }\end{array}$ & $\begin{array}{l}\% \text { SAMPLE } \\
\text { RECOVERY }\end{array}$ & $\begin{array}{c}\text { WI } \\
\text { MEAN }\end{array}$ & $\begin{array}{l}\text { SPEED } \\
\text { S.D. }\end{array}$ & $\begin{array}{l}(M / S) \\
\text { MAX }\end{array}$ \\
\hline 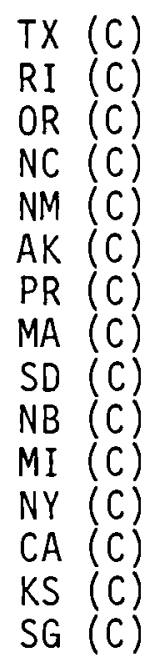 & $\begin{array}{r}9.1 \\
9.1 \\
9.1 \\
18.2 \\
9.1 \\
9.1 \\
9.1 \\
18.2 \\
9.1 \\
9.1 \\
18.2 \\
18.2 \\
9.1 \\
9.1 \\
9.1\end{array}$ & $\begin{array}{l}94.5 \\
83.3 \\
93.8 \\
96.6 \\
79.6 \\
63.4 \\
75.1 \\
93.2 \\
87.2 \\
81.4 \\
96.5 \\
98.2 \\
93.2 \\
84.5 \\
90.4\end{array}$ & $\begin{array}{l}5.8 \\
4.6 \\
3.8 \\
5.5 \\
5.3 \\
6.2 \\
6.5 \\
4.3 \\
4.5 \\
4.8 \\
5.0 \\
5.6 \\
4.7 \\
5.1 \\
5.6\end{array}$ & $\begin{array}{l}2.7 \\
2.2 \\
2.7 \\
3.8 \\
2.9 \\
3.7 \\
2.5 \\
2.3 \\
2.6 \\
2.5 \\
2.9 \\
3.3 \\
2.5 \\
2.6 \\
3.8\end{array}$ & $\begin{array}{l}17.7 \\
14.6 \\
34.9 \\
28.8 \\
21.3 \\
18.8 \\
29.1 \\
15.5 \\
16.2 \\
15.4 \\
19.3 \\
20.1 \\
16.0 \\
16.4 \\
18.7\end{array}$ \\
\hline $\begin{array}{l}\text { RI }(B) \\
\text { OR (B) } \\
N C \text { (B) } \\
N M \quad(B)\end{array}$ & $\begin{array}{l}30.0 \\
39.6 \\
45.7 \\
30.0\end{array}$ & $\begin{array}{l}55.2 \\
94.2 \\
89.1 \\
79.6\end{array}$ & $\begin{array}{l}5.7 \\
4.8 \\
7.0 \\
6.7\end{array}$ & $\begin{array}{l}2.6 \\
3.5 \\
3.9 \\
3.2\end{array}$ & $\begin{array}{l}16.2 \\
39.8 \\
29.9 \\
25.0\end{array}$ \\
\hline $\begin{array}{l}\operatorname{TX}(A) \\
\operatorname{RI}(A) \\
\operatorname{OR}(A) \\
\mathrm{NC}(A) \\
\mathrm{NM}(A) \\
\mathrm{AK}(A) \\
\operatorname{PR}(A) \\
\mathrm{MA}(A) \\
\mathrm{SD}(A) \\
\mathrm{NB}(A) \\
\operatorname{MI}(A) \\
\mathrm{NY}(A) \\
\mathrm{CA}(A) \\
\mathrm{KS}(A) \\
\mathrm{SG}(A)\end{array}$ & $\begin{array}{l}45.7 \\
45.7 \\
70.7 \\
76.2 \\
45.7 \\
21.8 \\
45.7 \\
45.7 \\
45.7 \\
45.7 \\
45.7 \\
45.7 \\
45.7 \\
45.7 \\
45.7\end{array}$ & $\begin{array}{l}94.5 \\
83.1 \\
94.3 \\
93.5 \\
79.6 \\
64.5 \\
74.7 \\
93.5 \\
87.3 \\
81.3 \\
95.7 \\
92.9 \\
86.2 \\
84.8 \\
90.0\end{array}$ & $\begin{array}{l}7.6 \\
7.0 \\
5.4 \\
7.8 \\
7.3 \\
7.1 \\
7.4 \\
6.5 \\
6.5 \\
6.2 \\
7.2 \\
6.7 \\
6.4 \\
7.0 \\
7.2\end{array}$ & $\begin{array}{l}3.3 \\
3.1 \\
3.9 \\
4.5 \\
3.3 \\
4.2 \\
2.6 \\
3.6 \\
3.2 \\
3.1 \\
3.7 \\
3.5 \\
3.4 \\
3.1 \\
5.2\end{array}$ & $\begin{array}{l}22.3 \\
21.5 \\
27.7 \\
34.6 \\
27.3 \\
22.9 \\
31.7 \\
25.5 \\
19.1 \\
18.8 \\
25.6 \\
21.4 \\
20.5 \\
19.1 \\
24.3\end{array}$ \\
\hline
\end{tabular}

Note: S.D. = Standard deviation

$$
\begin{aligned}
\text { MAX } & =\text { Maximum value } \\
A & =\text { Upper level } \\
B & =\text { Mid-l evel } \\
C & =\text { Lower level. }
\end{aligned}
$$


TABLE 4. Mean Annual Wind Speeds

Based on Monthly Means

\begin{tabular}{|c|c|c|c|c|c|}
\hline $\begin{array}{l}\text { SITE } \\
\text { CODE }\end{array}$ & $\begin{array}{l}\text { SENSOR } \\
\text { LEVEL (M) }\end{array}$ & $\begin{array}{c}\bar{V}(M / S) \\
1977\end{array}$ & $\begin{array}{c}\bar{V}(M / S) \\
1978\end{array}$ & $\begin{array}{c}\bar{V}(M / S) \\
1979\end{array}$ & $\begin{array}{c}\bar{V}(M / S) \\
\text { 3-YR } \\
\text { AVERAGE }\end{array}$ \\
\hline $\begin{array}{l}\text { TX (C) } \\
\text { RI (C) } \\
\text { OR (C) } \\
\text { NC (C) } \\
\text { NM (C) } \\
\text { AK (C) } \\
\text { PR (C) } \\
\text { MA (C) } \\
\text { SD (C) } \\
\text { NB (C) } \\
\text { MI (C) (C) } \\
\text { NY (C) } \\
\text { CA (C) } \\
\text { KS (C) } \\
\text { SG (C) }\end{array}$ & $\begin{array}{r}9.1 \\
9.1 \\
9.1 \\
18.2 \\
9.1 \\
9.1 \\
9.1 \\
18.2 \\
9.1 \\
9.1 \\
18.2 \\
18.2 \\
9.1 \\
9.1 \\
9.1\end{array}$ & $\begin{array}{l}6.5 \\
4.8 \\
4.5 \\
5.9 \\
5.4 \\
6.8 \\
6.3 \\
4.9 \\
5.0 \\
5.7 \\
4.4 \\
6.1 \\
5.2 \\
5.6 \\
6.5\end{array}$ & $\begin{array}{l}6.7 \\
5.0 \\
3.9 \\
5.6 \\
5.5 \\
7.5 \\
6.4 \\
4.7 \\
4.8 \\
5.6 \\
4.9 \\
6.1 \\
4.8 \\
5.7 \\
6.7\end{array}$ & $\begin{array}{l}5.8 \\
4.6 \\
3.8 \\
5.5 \\
5.3 \\
6.2 \\
6.5 \\
4.3 \\
4.5 \\
4.8 \\
5.0 \\
5.6 \\
4.7 \\
5.1 \\
5.6\end{array}$ & $\begin{array}{l}6.3 \\
4.8 \\
4.1 \\
5.7 \\
5.4 \\
6.8 \\
6.4 \\
4.6 \\
4.8 \\
5.4 \\
4.8 \\
5.9 \\
4.9 \\
5.5 \\
6.5\end{array}$ \\
\hline $\begin{array}{l}\text { RI (B) } \\
\text { OR (B) } \\
\text { NC (B) } \\
\text { NM (B) }\end{array}$ & $\begin{array}{l}30.0 \\
39.6 \\
45.7 \\
30.0\end{array}$ & $\begin{array}{l}\text { N/A } \\
5.9 \\
8.0 \\
\text { N/A }\end{array}$ & $\begin{array}{l}\text { N/A } \\
4.8 \\
7.7 \\
7.3(\mathrm{~b})\end{array}$ & $\begin{array}{l}5.7 \\
4.8 \\
7.0 \\
6.7\end{array}$ & $\begin{array}{l}5.7 \\
5.2 \\
7.6 \\
7.0\end{array}$ \\
\hline $\begin{array}{l}\text { TX (A) } \\
\text { RI (A) } \\
\text { OR (A) } \\
\text { NC (A) } \\
\text { NM (A) } \\
\text { AK (A) } \\
\text { PR (A) } \\
\text { MA (A) } \\
\text { SD (A) } \\
\text { NB (A) } \\
\text { MI (A) C } \\
\text { NY (A) } \\
\text { CA (A) } \\
\text { KS (A) } \\
\text { SG (A) }\end{array}$ & $\begin{array}{l}45.7 \\
45.7 \\
70.1 \\
76.2 \\
45.7 \\
21.8 \\
45.7 \\
45.7 \\
45.7 \\
45.7 \\
45.7 \\
45.7 \\
45.7 \\
45.7 \\
45.7\end{array}$ & $\begin{array}{l}8.3 \\
7.4 \\
\text { N/A } \\
\text { N/A } \\
7.4 \\
9.6 \\
6.9 \\
7.2 \\
6.7 \\
6.8 \\
7.5 \\
7.2 \\
6.7 \\
7.5 \\
7.9\end{array}$ & $\begin{array}{l}8.0 \\
7.4 \\
\text { N/A } \\
7.0(a) \\
7.4 \\
8.3 \\
7.1 \\
7.1 \\
6.6 \\
7.0 \\
7.8 \\
7.4 \\
6.8 \\
7.5 \\
8.1\end{array}$ & $\begin{array}{l}7.6 \\
7.0 \\
5.4 \\
7.8 \\
7.3 \\
7.1 \\
7.4 \\
6.5 \\
6.5 \\
6.2 \\
7.2 \\
6.7 \\
6.4 \\
7.0 \\
7.2\end{array}$ & $\begin{array}{l}8.0 \\
7.3 \\
5.4 \\
7.4 \\
7.4 \\
8.3 \\
7.1 \\
6.9 \\
6.6 \\
6.7 \\
7.5 \\
7.1 \\
6.6 \\
7.3 \\
7.7\end{array}$ \\
\hline
\end{tabular}

(a) Data reporting began in July of 1978

(b) Data reporting began in March of 1978

(c) Collection program terminated on November 3, 1979 $\mathrm{N} / \mathrm{A}=$ Not available. 


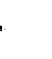

, 


\section{SUMMARY TABLES}

Summarized hourly data for each site are presented in eight tables and one figure. The hourly data are obtained by averaging the 2 -min instantaneous data that are routinely collected at the site. A valid hourly value was calculated from at least 10 valid 2 -min values acquired during the hour.

Discussion of data represented in each table and the method of calculation is given below. The notation "W/S" refers to wind speed, which is reported in meters per second. The notation "W/D" refers to wind direction, which is reported in degrees. Information on site elevation is reported in meters.

A value reported as $\mathbf{- 9 9 9 . 9 9}$ indicates missing data. At several sites no mid-level sensors exist. This condition is noted at the bottom of Table 1.3. The values for the mid-level wind speed in Table 1.3 under the column "Other Levels" are therefore indicated as missing, -999.99. If no mid-level sensors exist, the values reported for Level $B$ in Tables 1.4, 1.5, and 1.6 are zero. Also, no information for Level $B$ is provided in Tables 1.1 and 1.2 .

\section{TITLE AND FUNCTION}

Table 1.1

Sensor Performance. Sensor performance identifies the percentage of time the recording system was operating and the percentage of time acceptable data were acquired by the meteorological sensors. The values given under the label "\% On-Line" are obtained by dividing the total number of data scans collected by the total possible number of data scans. The values given under the label "\% Recovered" are obtained by dividing the total number of acceptable data scans collected by the total possible number of data scans.

Table 1.2

Annual Means and Standard Deviations. This table provides the mean wind speed, wind direction, and standard deviation of wind speed for each available sensor level during the reporting period. The value for wind direction indicates the direction from which the wind blows with $0^{\circ}$ and $360^{\circ}$ being north, $90^{\circ}$ being east, $180^{\circ}$ being south and $270^{\circ}$ being west. Thus, a mean wind 
direction of $220^{\circ}$ indicates winds that blow primarily from the southwest. The value reported for standard deviation of wind speed indicates the range, both positive and negative of mean wind speed values that would include about $68 \%$ of all observed wind speed values for that reporting level.

Table 1.3

Maximum Wind Speed. The highest calculated hourly value for each available sensor level is given along with date and time of occurrence. For this maximum hourly wind speed value, the mean hourly wind direction is given along with the hourly wind speeds that occurred at the other levels.

Table 1.4

Wind Speed and Direction Versus Time-of-Day. Mean hourly wind speed and direction values are reported for each hour of the day for the reporting period. Data reported for each hour represent the average of all hourly values calculated for that hour. Data are provided for each level of instrumentation. If the site has no sensor at the mid-level (B), the values for wind speed and wind direction are reported as 0.0 .

Table 1.5

Frequency Distribution of Wind Speed. For each sensor level, the total number of observations and percentage of occurrences are given for a number of classes of wind speed. The first class, 0.0 to 0.5 , represents winds from 0.0 up to and including $0.5 \mathrm{~m} / \mathrm{s}$. For the rest of the classes, except the last, the number under the "Count" column indicates the number of occurrences of hourly wind speeds greater than the lower limit and less than or equal to the upper limit. Thus, the class 0.5 to 1.0 indicates the condition $0.5<$ wind speed $(\mathrm{m} / \mathrm{s}) \leq 1.0$. The recovery rate, in percent, for each wind speed sensor is given at the bottom of the table. If no mid-level sensor is available, all values under Level $B$ and the recovery rate for Level $B$ will be 0 or 0.0 .

Table 1.6

Cumulative Frequency Distribution of Wind Speed. Both the cumulative number of observations and percentage of cumulative frequency distribution of 
hourly wind speed values are given for various wind speed intervals. The value of 2.0 under the column "wind speed" represents wind speed occurrences up to and including $2.0 \mathrm{~m} / \mathrm{s}$. The corresponding number of cumulative hourly wind speed values is noted under the column "CFD" for each sensor level. If the site has no mid-level sensor, the values for Level B under the columns "CFD(ABS)" and "CFD(\%)" will be 0 and 0.0 , respectively.

Table 1.7

Wind Speed Persistence Frequency. Information presented in this table indicates the length of time the wind speed at the upper sensor level ( $A$ ) occurs within a specified wind speed interval. For example, if in the "Hours" row of 2.0 and under the wind speed column of 4 to $12 \mathrm{~m} / \mathrm{s}$ a value of 3 was reported, this indicates that during the reporting period there were three occurrences of wind speeds between 4 and $12 \mathrm{~m} / \mathrm{s}$ that lasted for two hours.

Table 1.8

Power Law Exponent. A power law exponent, expressed as "ALPHA," between sensor levels is computed as indicated in the notes at the bottom of the table for each hour of data. These values are summed according to the wind direction occurring at the upper sensor level. After all data has have analyzed for the reporting period, mean values of "ALPHA" are computed according to the total number of values of "ALPHA" summed for each directional sector. The purpose of this type of data representation is to investigate how the topographical features surrounding the site may influence general wind flow characteristics.

The value under the column $\operatorname{ALPHA}(A, B, C)$ represents a best fit of the values for $\operatorname{ALPHA}(A, B), \operatorname{ALPHA}(B, C)$, and $\operatorname{ALPHA}(A, C)$. These values are only computed for sites with three levels of sensors. If the site has two levels of instrumentation, i.e., no Level $B$ sensor, no best fit value will be calculated and information on ALPHA will be found under the column "ALPHA $(A, C)$."

The percentage of occurrence of wind direction for each sensor is also given in this table. That information is provided under the columns "\%A," "\%," "\%" for the upper, mid-, and lower sensor levels, respectively. 
AMARILLO, TEXAS 
$\begin{array}{ll}\text { SITE IO: } & \text { IX } \\ \text { SITE LOCATION: AMARILLO, TX. } & \\ \text { DATA : } & \text { JANUAKY }\end{array}$

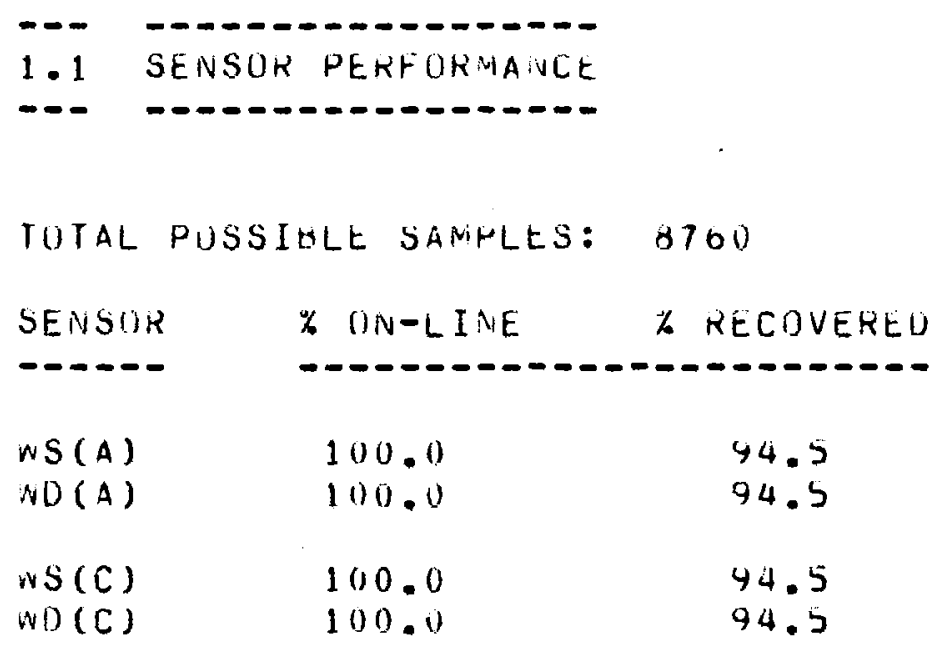

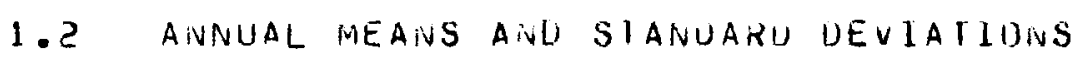

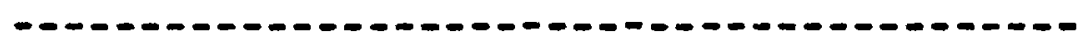

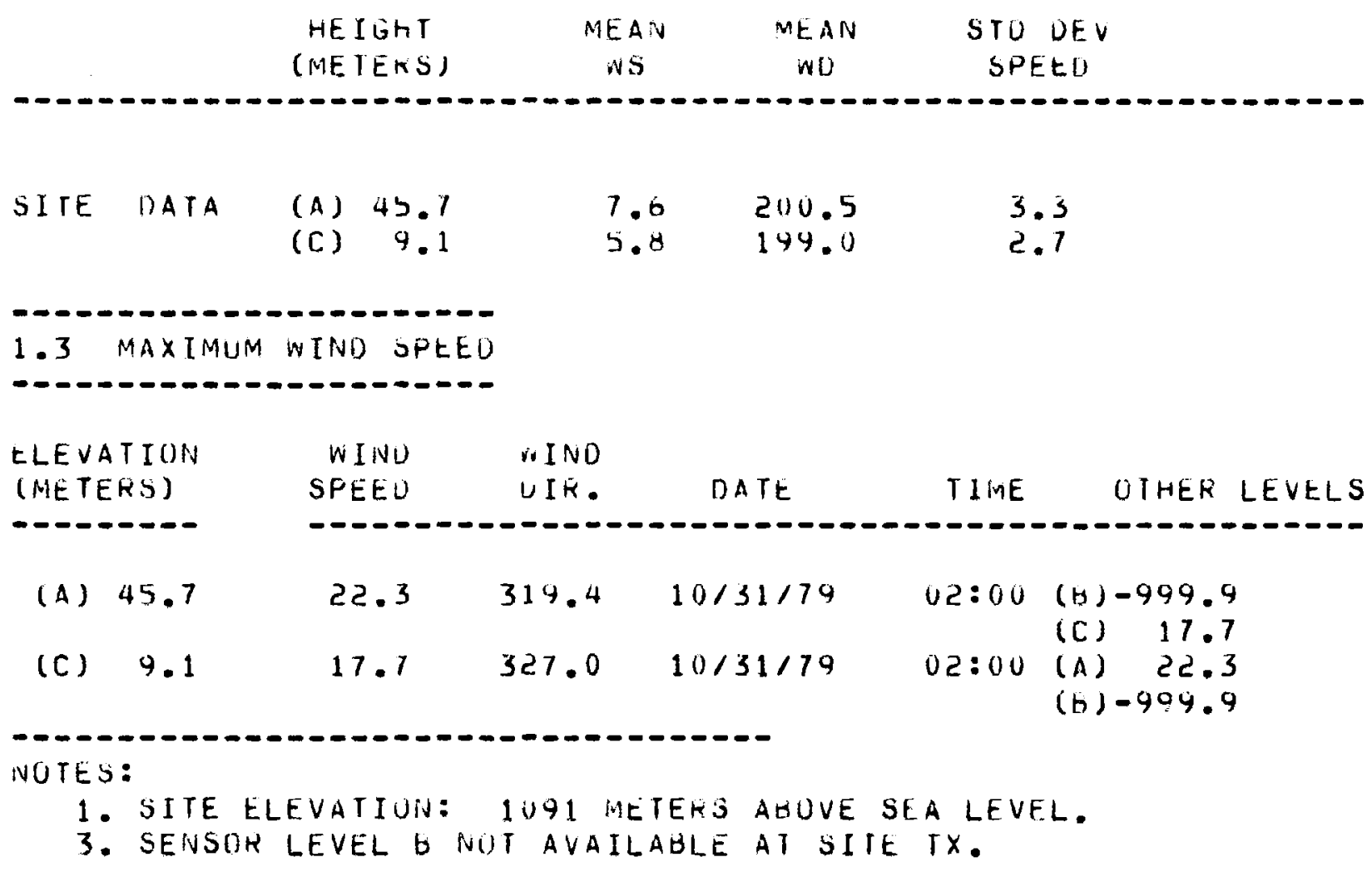


SITE IO: IX

STIE LUCAIIUN: AMARILLO, TX.

UATA : JAMUAKY 1979 THKWUGH LECEMAER 1979

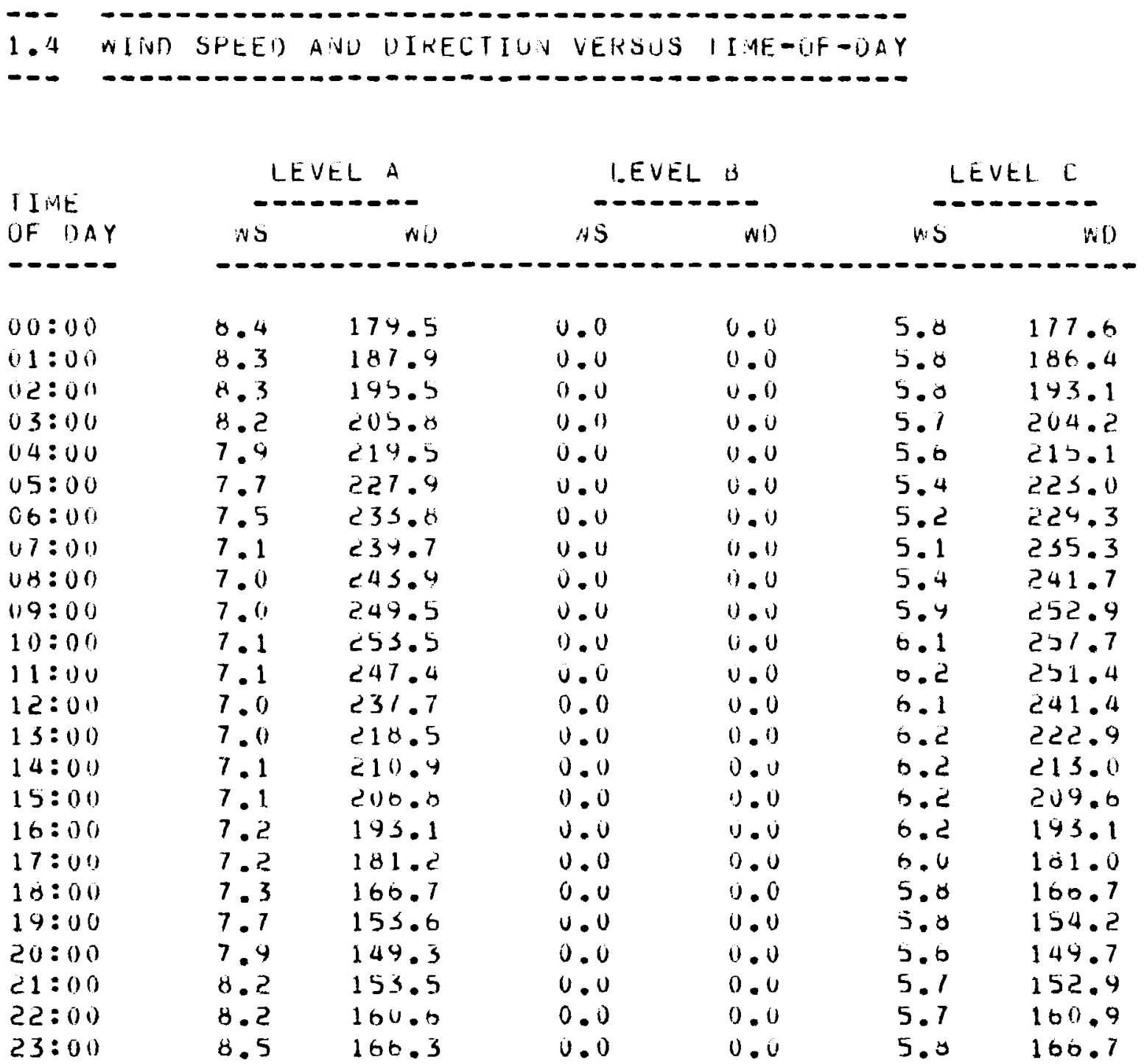


SITE 10: IX

DITE LUCATIUN: AMARILLO, IX.

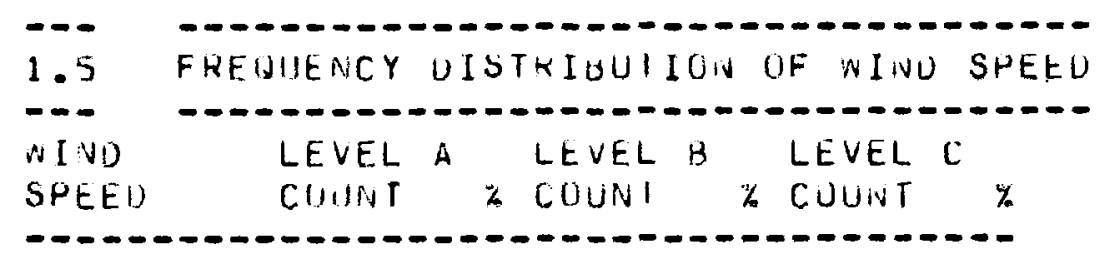

$\begin{array}{rrrrrrr}0.0-0.5 & 6 & 0.1 & 0 & 0.0 & 9 & 0.1 \\ 0.5-1.0 & 30 & 0.4 & 0 & 0.0 & 58 & 0.7 \\ 1.0-1.5 & 71 & 0.9 & v & 0.0 & 155 & 1.9 \\ 1.5-2.0 & 127 & 1.5 & 0 & 0.0 & 275 & 3.3 \\ 2.0-2.5 & 172 & 2.1 & 0 & 0.0 & 420 & 5.1 \\ 2.5-3.0 & 226 & 2.7 & 0 & 0.0 & 450 & 5.2 \\ 3.0-3.5 & 271 & 3.3 & 0 & 0.0 & 504 & 6.1 \\ 3.5-4.0 & 302 & 3.0 & 0 & 0.0 & 540 & 0.5 \\ 4.0-4.5 & 385 & 4.7 & 0 & 0.0 & 533 & 6.4 \\ 4.5-5.0 & 434 & 5.2 & 0 & 0.0 & 627 & 1.6 \\ 5.0-5.5 & 435 & 5.3 & 0 & 0.0 & 538 & 0.5 \\ 5.5-6.0 & 410 & 5.0 & 0 & 0.0 & 540 & 6.6 \\ 6.0-6.5 & 430 & 5.2 & 0 & 0.0 & 561 & 6.9 \\ 6.5-7.0 & 419 & 5.1 & 0 & 0.0 & 507 & 6.1 \\ 7.0-7.5 & 443 & 5.4 & 0 & 0.0 & 477 & 5.8 \\ 7.5-8.0 & 470 & 5.7 & 0 & 0.0 & 413 & 5.0 \\ 8.0-8.5 & 447 & 5.4 & 0 & 0.0 & 347 & 4.2 \\ 8.5-9.0 & 453 & 5.5 & 0 & 0.0 & 206 & 3.5 \\ 9.0-9.5 & 448 & 5.4 & 0 & 0.0 & 227 & 2.7 \\ 9.5-10.0 & 385 & 4.7 & 0 & 0.0 & 203 & 2.5 \\ 10.0-11.0 & 652 & 7.9 & 0 & 0.0 & 264 & 3.2 \\ 11.0-12.0 & 495 & 0.0 & 0 & 0.0 & 157 & 1.9 \\ 12.0-13.0 & 300 & 3.6 & 0 & 0.0 & 88 & 1.1 \\ 13.0-14.0 & 199 & 2.4 & 0 & 0.0 & 48 & 0.6 \\ 14.0-15.0 & 111 & 1.3 & 0 & 0.0 & 28 & 0.3 \\ 15.0-10.0 & 65 & 0.8 & 0 & 0.0 & 21 & 0.3 \\ 16.0-17.0 & 45 & 0.5 & 0 & 0.0 & 5 & 0.1 \\ 17.0-18.0 & 23 & 0.3 & 0 & 0.0 & 3 & 0.0 \\ 18.0-19.0 & 11 & 0.1 & 0 & 0.0 & 0 & 0.0 \\ 19.0-20.0 & 5 & 0.1 & 0 & 0.0 & 0 & 0.0 \\ 20.0-21.0 & 1 & 0.0 & 0 & 0.0 & 0 & 0.0 \\ >21.0 & 3 & 0.0 & 0 & 0.0 & 0 & 0.0\end{array}$

LEVEL A LEVEL 3 LEVEL C

$$
94.5 \quad 0.0 \quad 94.5
$$


SITE II): TX

STTE LOCATIUN: AMAKLLLO, TX.

UATA : JANUAKY 1479 THRULGH UECENGER 1979

1.6 CIMIILATIVE FREUUENCY UISTRIBUTIUN UF WINO SFEEO

-- - - - - - - - - - - - - - - - - - - - -

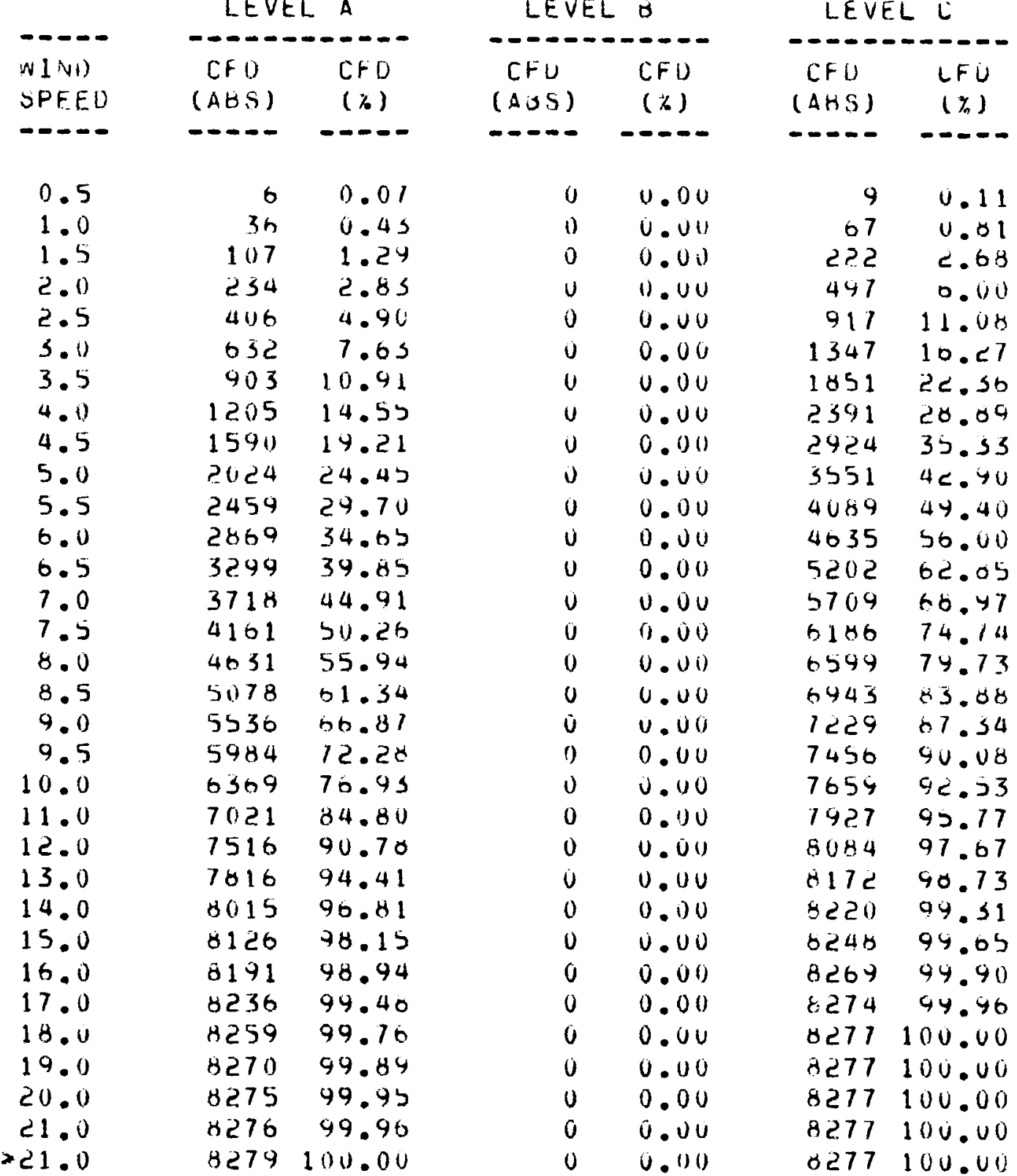




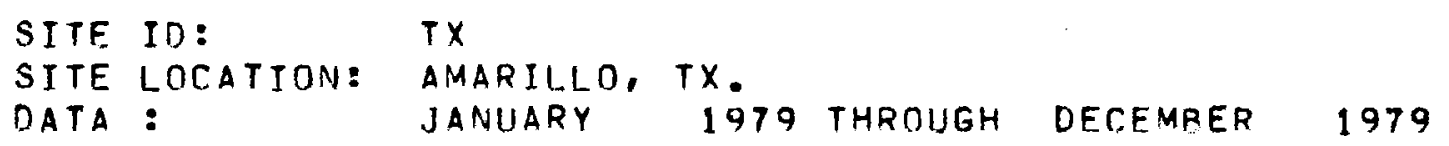

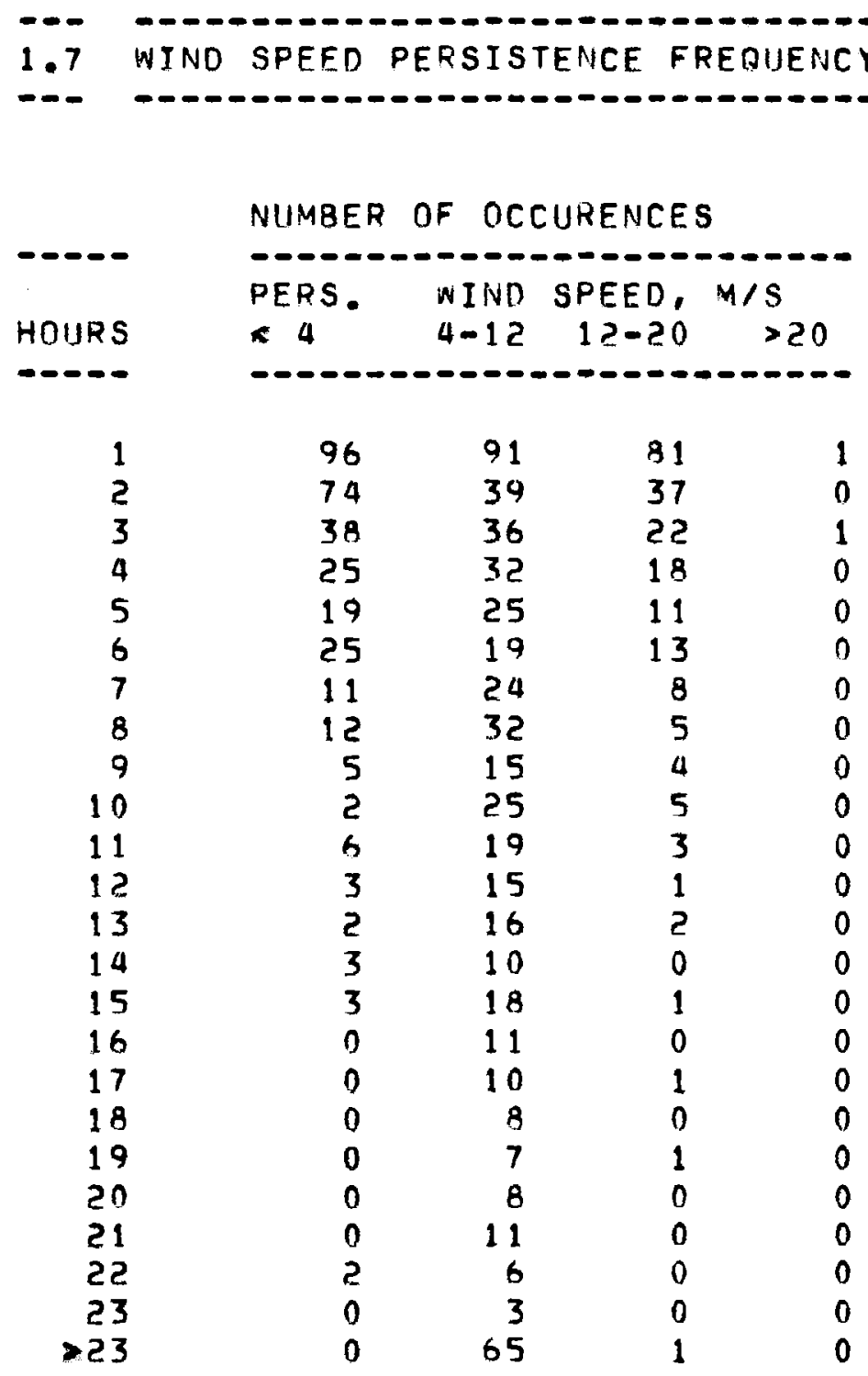

(LEVEL A) 


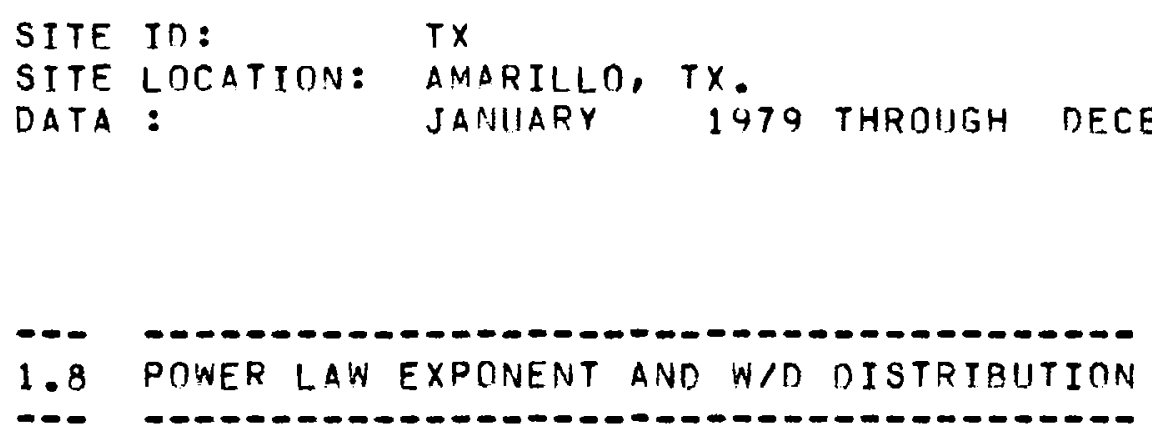

\begin{tabular}{|c|c|c|c|c|c|c|c|}
\hline$(E L E V A)$ & $\begin{array}{l}A L P H A \\
(A, B)\end{array}$ & $\begin{array}{l}A L P H A \\
(B, C)\end{array}$ & $\begin{array}{l}A L P H A \\
(A, C)\end{array}$ & $\begin{array}{l}A L P H A \\
(A, B, C)\end{array}$ & KA & $\% 8$ & $\pi \mathrm{C}$ \\
\hline$N$ & 0.00 & 0.00 & 0.12 & 0.00 & 5.23 & 0.00 & 4.52 \\
\hline NNE & 0.00 & 0.00 & 0.11 & 0.00 & 7.11 & 0.00 & 7.27 \\
\hline NE & 0.00 & 0.00 & 0.19 & 0.00 & 3.48 & 0.00 & 3.81 \\
\hline ENE & 0.00 & 0.00 & 0.16 & 0.00 & 2.74 & 0.00 & 2.68 \\
\hline E & 0.00 & 0.00 & 0.19 & 0.00 & 2.50 & 0.00 & 2.42 \\
\hline ESE & 0.00 & 0.00 & 0.17 & 0.00 & 3.09 & 0.00 & 3.02 \\
\hline SE & 0.00 & 0.00 & 0.19 & 0.00 & 6.33 & 0.00 & 6.39 \\
\hline SSE & 0.00 & 0.00 & 0.23 & 0.00 & 11.14 & 0.00 & 11.66 \\
\hline $\mathrm{s}$ & 0.00 & 0.00 & 0.15 & 0.00 & 11.47 & 0.00 & 11.74 \\
\hline SSIN & 0.00 & 0.00 & 0.13 & 0.00 & 13.25 & 0.00 & 12.43 \\
\hline$S W$ & 0.00 & 0.00 & 0.18 & 0.00 & 9.75 & 0.00 & 10.17 \\
\hline WSW & 0.00 & 0.00 & 0.18 & 0.00 & 6.29 & 0.00 & 6.83 \\
\hline$w$ & $0.0 n$ & 0.00 & 0.19 & 0.00 & 4.44 & 0.00 & 4.51 \\
\hline WNW & 0.00 & 0.00 & $n .24$ & 0.00 & 4.52 & 0.00 & 4.14 \\
\hline NW & 0.0 & 0.00 & 0.20 & 0.00 & 4.05 & 0.00 & 4.14 \\
\hline NNW & 0.00 & 0.00 & 0.12 & 0.00 & 4.59 & 0.00 & 4.26 \\
\hline
\end{tabular}

NOTES:

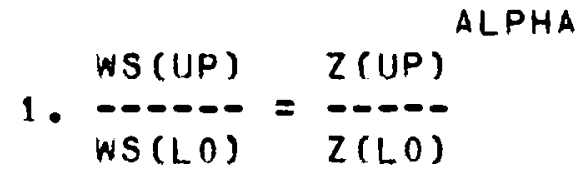

$$
\begin{aligned}
& \begin{array}{l}
\text { 2. ALPHA }=\frac{\text { LOG(WS(UP)/WS(LO)) }}{\text { LOG(Z(UP)/Z(LO)) WHERE; } \quad \text { Z=ELEVATION }} \text { WS=WIND SPEED }
\end{array}
\end{aligned}
$$


COUNT OF WIND SPEED VS. DIRECTION

EACH LINE OF CHAARCTERS REPRESENTS 40 OBSERVATIONS

SPEED INCREASES WITH DISTRNCE FROM CENTER OF CIRCLES

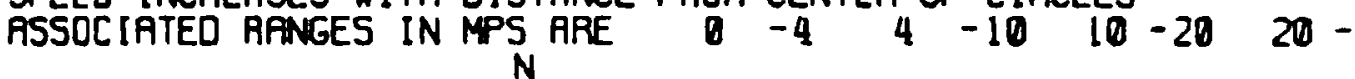

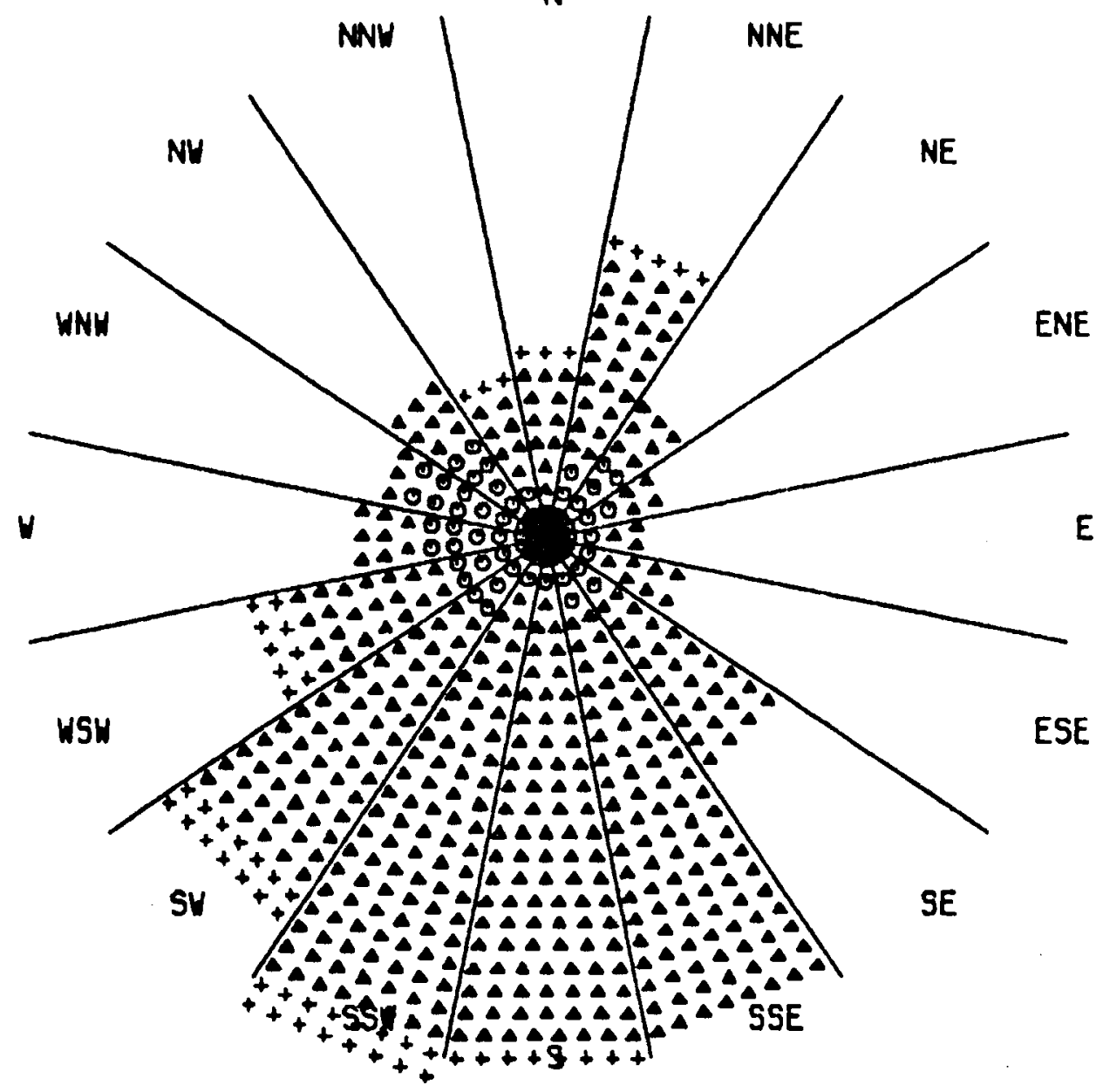

FIGURE 1. (TX) Wind Rose for Lowest Sensor Level 


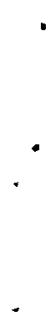


BLOCK ISLAND, RHODE ISLAND 


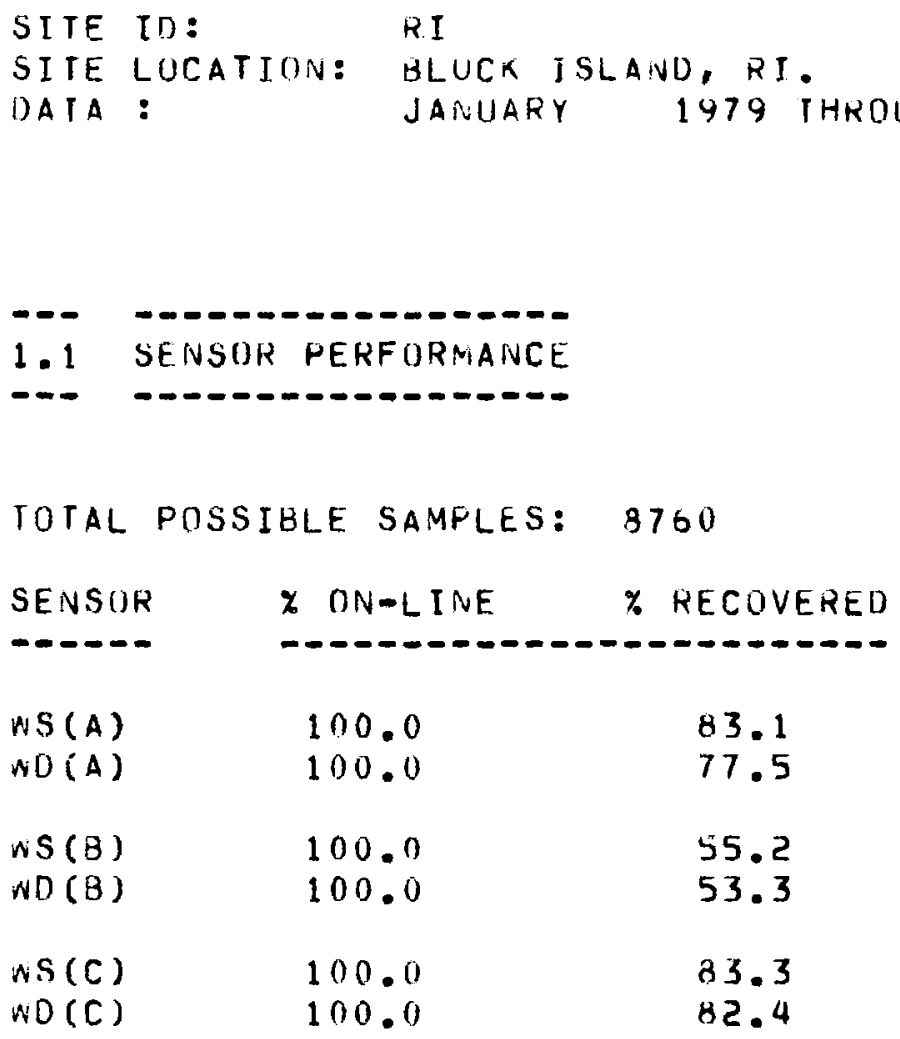

1.2 ANNUAL MEANS AIVD SIANDARU DEVIATIONS

1.

$\begin{array}{ccccc}\text { HEIGHT } & \text { MEAN } & \text { MEAN } & \text { STD UEV } \\ \text { (METEKS) } & \text { WS } & \text { ND } & \text { SPEEU } \\ \text { SITE DATA } & \text { (A) } 45.7 & 7.0 & 253.5 & 3.1 \\ & \text { (B) } 30.0 & 5.7 & 240.3 & 2.6 \\ & \text { (C) } 9.1 & 4.0 & 252.0 & 2.2\end{array}$

1.3 MAXIMUM ININU SPEEU

--- - - - - - - - - - - - - - - - -

\begin{tabular}{|c|c|c|c|c|c|c|}
\hline \multicolumn{2}{|c|}{$\begin{array}{l}\text { ELEVATION } \\
\text { (METERS) }\end{array}$} & $\begin{array}{l}\text { WINU } \\
\text { SPEEU }\end{array}$ & $\begin{array}{l}\text { WIND } \\
\text { UIR. }\end{array}$ & DATE & TIME & OTHEK \\
\hline$---\infty$ & 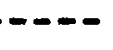 & $-\cdots$ & & & & \\
\hline (A) & 45.7 & 21.5 & 142.1 & $01 / 25 / 79$ & $01: 00$ & $\begin{array}{l}\text { (B) }-999.9 \\
\text { (C) } 14.6\end{array}$ \\
\hline (B) & 30.0 & 16.2 & $3 B .6$ & $08 / 12 / 79$ & $16: 00$ & $\begin{array}{l}\text { (A) } 17.4 \\
\text { (C) } 11.6\end{array}$ \\
\hline (C) & 9.1 & 14.6 & 131.3 & $01 / 25 / 79$ & $01: 00$ & $\begin{array}{l}\text { (A) } 21.5 \\
\text { (B) }-999.9\end{array}$ \\
\hline
\end{tabular}

NOTES:

1. Site elevatiun: 14 METERS above Sea leVel. 
SITE IOU:

SITE LUCATIOIN: BLUCK ISLAND, RI.

CATA:
RI

JAIVUARY

\begin{tabular}{|c|c|c|c|c|c|c|}
\hline $\begin{array}{l}\text { IIME } \\
\text { OF DAY }\end{array}$ & wS & $\begin{array}{c}L A \\
\text { WD }\end{array}$ & is & $\begin{array}{l}E L \\
-N()\end{array}$ & ns & $\frac{L C}{W D}$ \\
\hline------ & & & & & & $=-$ \\
\hline $00: 00$ & 7.2 & 265.7 & 5.7 & 249.3 & 4.4 & 266.4 \\
\hline $01: 00$ & 7.1 & 275.1 & 5.6 & 254.2 & 4.5 & 274.4 \\
\hline $0 ح: 00$ & 6.9 & 280.2 & 5.5 & 257.8 & 4.3 & 273.3 \\
\hline $03: 00$ & 6.8 & 286.2 & 5.4 & 260.2 & 4.2 & 278.1 \\
\hline $04: 00$ & 6.8 & 289.1 & 5.4 & 209.1 & 4.2 & 280.3 \\
\hline $05: 00$ & 6.7 & 294.4 & 5.4 & 271.8 & 4.1 & 277.2 \\
\hline U6:00 & 6.7 & 294.9 & 5.3 & 273.8 & 4.2 & 280.8 \\
\hline $07: 00$ & 6.6 & 296.3 & 5.3 & 270.1 & 4.4 & 294.3 \\
\hline $08: 00$ & 6.6 & 307.4 & 5.5 & 287.0 & 4.6 & 313.0 \\
\hline $09: 00$ & 6.5 & 304.6 & 5.5 & 289.5 & 4.8 & 315.6 \\
\hline $10: 00$ & 6.5 & 279.0 & 5.6 & 275.4 & 4.9 & 295.2 \\
\hline $11: 00$ & 6.6 & 262.0 & 5.7 & 251.9 & 4.9 & 262.8 \\
\hline $12: 00$ & 6.7 & 238.6 & 5.8 & 229.7 & 5.0 & 240.3 \\
\hline $13: 00$ & 6.9 & 230.7 & 5.9 & 219.9 & 5.1 & 232.7 \\
\hline $14: 00$ & 7.1 & 225.6 & 6.1 & 213.8 & 5.2 & 227.0 \\
\hline $15: 00$ & 7.2 & 224.0 & 6.1 & 211.8 & 5.1 & 224.2 \\
\hline $16: 00$ & 7.3 & 222.1 & 6.1 & 211.0 & 5.0 & 222.6 \\
\hline $17: 00$ & 7.4 & 224.4 & 6.1 & 214.4 & 4.9 & 224.1 \\
\hline $18: 00$ & 7.5 & 226.2 & 6.0 & 218.5 & 4.8 & 226.5 \\
\hline $19: 00$ & 7.5 & 229.3 & 6.0 & 221.6 & 4.7 & 250.3 \\
\hline $20: 00$ & 7.5 & 23 . 0 & 5.9 & 227.1 & 4.0 & 234.9 \\
\hline $21: 00$ & 7.5 & 242.4 & 5.9 & 230.9 & 4.5 & 241.2 \\
\hline $22: 00$ & 7.4 & 251.5 & 5.9 & 236.0 & 4.5 & 248.4 \\
\hline $23: 00$ & 7.4 & 259.5 & 5.9 & 241.8 & 4.5 & 256.2 \\
\hline
\end{tabular}


$\begin{array}{llll}\text { SITE ID: } & \text { RI } & & \\ \text { SITE LOCATION: } & \text { GLOCK ISLANU, RI } & \\ \text { DATA : } & \text { JANUARY } 1979 \text { THROUGH } & \text { DECEMHER } & 1979\end{array}$

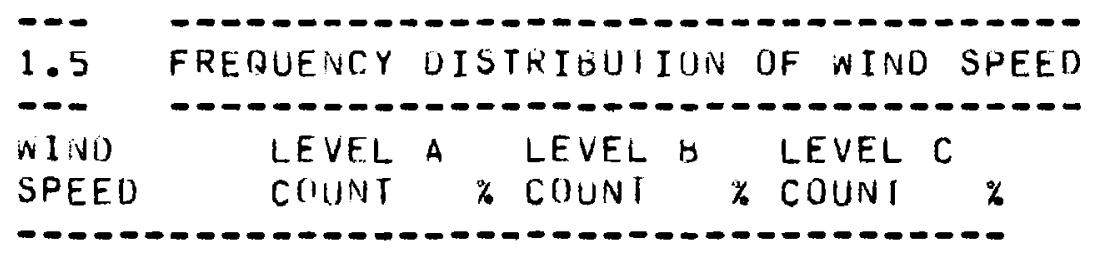

$\begin{array}{rrrrrrr}0.0-0.5 & 7 & 0.1 & 30 & 0.3 & 2 & 0.0 \\ 0.5-1.0 & 33 & 0.5 & 35 & 0.7 & 126 & 1.7 \\ 1.0-1.5 & 78 & 1.1 & 58 & 1.2 & 252 & 3.5 \\ 1.5-2.0 & 123 & 1.7 & 102 & 2.1 & 382 & 5.2 \\ 2.0-2.5 & 188 & 2.6 & 187 & 3.9 & 492 & 6.7 \\ 2.5-3.0 & 247 & 3.4 & 249 & 5.2 & 532 & 7.3 \\ 3.0-3.5 & 281 & 3.9 & 297 & 6.1 & 606 & 8.3 \\ 3.5-4.0 & 321 & 4.4 & 355 & 7.3 & 689 & 9.4 \\ 4.0-4.5 & 349 & 4.8 & 314 & 6.6 & 733 & 10.0 \\ 4.5-5.0 & 417 & 5.7 & 395 & 8.2 & 697 & 9.6 \\ 5.0-5.5 & 426 & 5.9 & 414 & 8.6 & 574 & 7.9 \\ 5.5-6.0 & 437 & 6.0 & 387 & 8.0 & 476 & 6.5 \\ 6.0-6.5 & 419 & 5.8 & 357 & 7.4 & 404 & 5.5 \\ 6.5-7.0 & 480 & 6.6 & 312 & 6.5 & 276 & 3.8 \\ 7.0-7.5 & 450 & 6.2 & 275 & 5.7 & 248 & 3.4 \\ 7.5-8.0 & 433 & 6.0 & 208 & 4.3 & 238 & 3.3 \\ 8.0-8.5 & 410 & 5.6 & 173 & 3.6 & 152 & 2.1 \\ 8.5-9.0 & 377 & 5.2 & 136 & 2.8 & 107 & 1.5 \\ 9.0-9.5 & 294 & 4.0 & 122 & 2.5 & 107 & 1.5 \\ 9.5-10.0 & 278 & 3.8 & 95 & 2.0 & 60 & 0.8 \\ 10.0-11.0 & 454 & 6.2 & 130 & 2.7 & 79 & 1.1 \\ 11.0-12.0 & 331 & 4.5 & 81 & 1.7 & 36 & 0.5 \\ 12.0-13.0 & 168 & 2.3 & 52 & 1.1 & 19 & 0.3 \\ 13.0-14.0 & 115 & 1.6 & 20 & 0.5 & 7 & 0.1 \\ 14.0-15.0 & 69 & 0.9 & 21 & 0.4 & 1 & 0.0 \\ 15.0-16.0 & 44 & 0.6 & 7 & 0.1 & 0 & 0.0 \\ 16.0-17.0 & 28 & 0.4 & 2 & 0.0 & 0 & 0.0 \\ 17.0-18.0 & 12 & 0.2 & 0 & 0.0 & 0 & 0.0 \\ 18.0-19.0 & 6 & 0.1 & 0 & 0.0 & 0 & 0.0 \\ 19.0-20.0 & 00 & 0.0 & 0 & 0.0 & 0 & 0.0 \\ 20.0-21.0 & 0 & 0.0 & 0 & 0.0 & 0 & 0.0 \\ 191.0 & 1 & 0.0 & 0 & 0.0 & 0 & 0.0\end{array}$

RECUVERY RATES

LEVEL A LEVEL $B$ LEVEL C

$83.1 \quad 55.2 \quad 83.3$ 
SITE ID: RI

SITE LUCATION: BLOCK ISLAND, RI.

UATA : JANUARY 1979 THKOUGH DECEMBER 1979

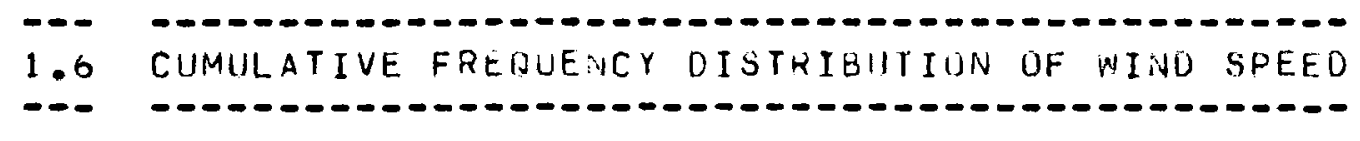

\begin{tabular}{|c|c|c|c|c|c|c|}
\hline & LEVE & $\begin{array}{l}\mathrm{L} \\
\mathrm{-}\end{array}$ & $\begin{array}{r}\text { LEV } \\
----\end{array}$ & $\begin{array}{l}\mathrm{B} \\
------\end{array}$ & LEV & $\begin{array}{l}\mathrm{L} \\
---\infty\end{array}$ \\
\hline $\begin{array}{l}\text { NIND } \\
\text { SPEED }\end{array}$ & $\begin{array}{l}C F D \\
(A B S)\end{array}$ & $\begin{array}{l}\text { CFD } \\
(\%)\end{array}$ & $\begin{array}{l}C F D \\
(A B S)\end{array}$ & $\begin{array}{l}\text { CFU } \\
(\%)\end{array}$ & $\begin{array}{l}(F D) \\
(A B S)\end{array}$ & $\begin{array}{l}C F D \\
(\%)\end{array}$ \\
\hline --- & $---\infty$ & $---\infty$ & $--\infty$ & $----\infty$ & $-\infty-$ & $-\cdot--$ \\
\hline 0.5 & 7 & 0.10 & 38 & 0.79 & 2 & 0.03 \\
\hline 1.0 & 40 & 0.55 & 73 & 1.51 & 128 & 1.75 \\
\hline 1.5 & 118 & 1.62 & 131 & 2.71 & $3 \hat{80}$ & 5.21 \\
\hline 2.0 & 241 & 3.31 & 233 & 4.82 & 762 & 10.45 \\
\hline 2.5 & 429 & 5.90 & 420 & 8.69 & 1254 & 17.19 \\
\hline 3.0 & 676 & 9.29 & 669 & 13.84 & 1786 & 24.48 \\
\hline 3.5 & 957 & 13.15 & 966 & 19.99 & 2392 & 32.79 \\
\hline 4.0 & 1278 & 17.56 & 1321 & 27.33 & 3081 & 42.23 \\
\hline 4.5 & 1627 & 22.36 & 1640 & 33.93 & 3814 & 52.20 \\
\hline 5.0 & 2044 & 28.09 & 2035 & 42.11 & 4511 & 61.84 \\
\hline 5.5 & 2470 & 33.95 & 2449 & 50.67 & 5085 & 69.71 \\
\hline 6.0 & 2907 & 39.95 & 2836 & 58.68 & 5561 & 76.23 \\
\hline 6.5 & 3326 & 45.71 & 3193 & 66.07 & 5965 & 81.77 \\
\hline 7.0 & 3806 & 52.31 & 3505 & 72.52 & 6241 & 85.55 \\
\hline 7.5 & 4256 & 58.49 & 3700 & 78.21 & 1589 & 88.95 \\
\hline 8.0 & 4689 & 64.44 & 3988 & 82.52 & 6727 & 92.21 \\
\hline 0.5 & 5099 & 70.08 & 4161 & 86.10 & 6879 & 94.30 \\
\hline 9.0 & 5476 & 75.26 & 4297 & 88.91 & 6986 & 95.76 \\
\hline 9.5 & 5770 & 79.30 & 4419 & 91.43 & 7093 & 97.23 \\
\hline 10.0 & 6048 & 83.12 & 4514 & 93.40 & 7153 & 98.05 \\
\hline 11.0 & $650 ?$ & 89.36 & 4644 & 96.09 & 7232 & 99.14 \\
\hline 12.0 & 6833 & 93.91 & 4725 & 97.77 & 7268 & 99.03 \\
\hline 13.0 & 7001 & 96.22 & 4777 & 98.84 & 7287 & 99.89 \\
\hline 14.0 & 7116 & 97.80 & 4803 & 99.38 & 7294 & 99.99 \\
\hline 15.0 & 7185 & 98.75 & 4824 & 99.81 & 7295 & 100.00 \\
\hline 16.0 & 7229 & 99.35 & 4851 & 99.96 & 7295 & 100.00 \\
\hline 17.0 & 7257 & 99.74 & 4833 & 100.00 & 7295 & 100.00 \\
\hline 18.0 & 7269 & 99.90 & 4833 & 100.00 & 7295 & 100.00 \\
\hline 19.0 & 7275 & 99.99 & 4833 & 100.00 & 7295 & 100.00 \\
\hline 20.0 & 7275 & 99.99 & 4833 & 100.00 & 7295 & 100.00 \\
\hline 21.0 & 7275 & 99.99 & 4833 & 100.00 & 7295 & 100.00 \\
\hline 21.0 & 7276 & 100.00 & 4833 & 100.00 & 7295 & 100.00 \\
\hline
\end{tabular}


SITE TO:

RI

SITE LOCATION: BLOCK ISLAND, RI.

DATA : JANUARY 1979 THROUGH DECEMBER 1979

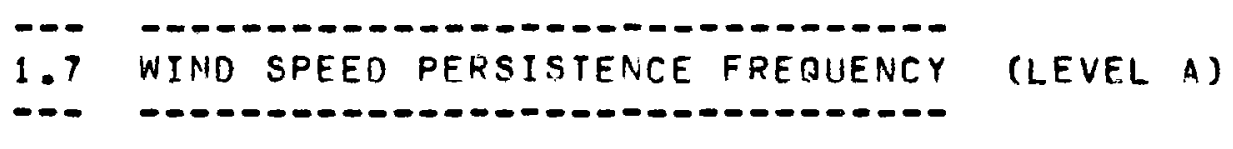

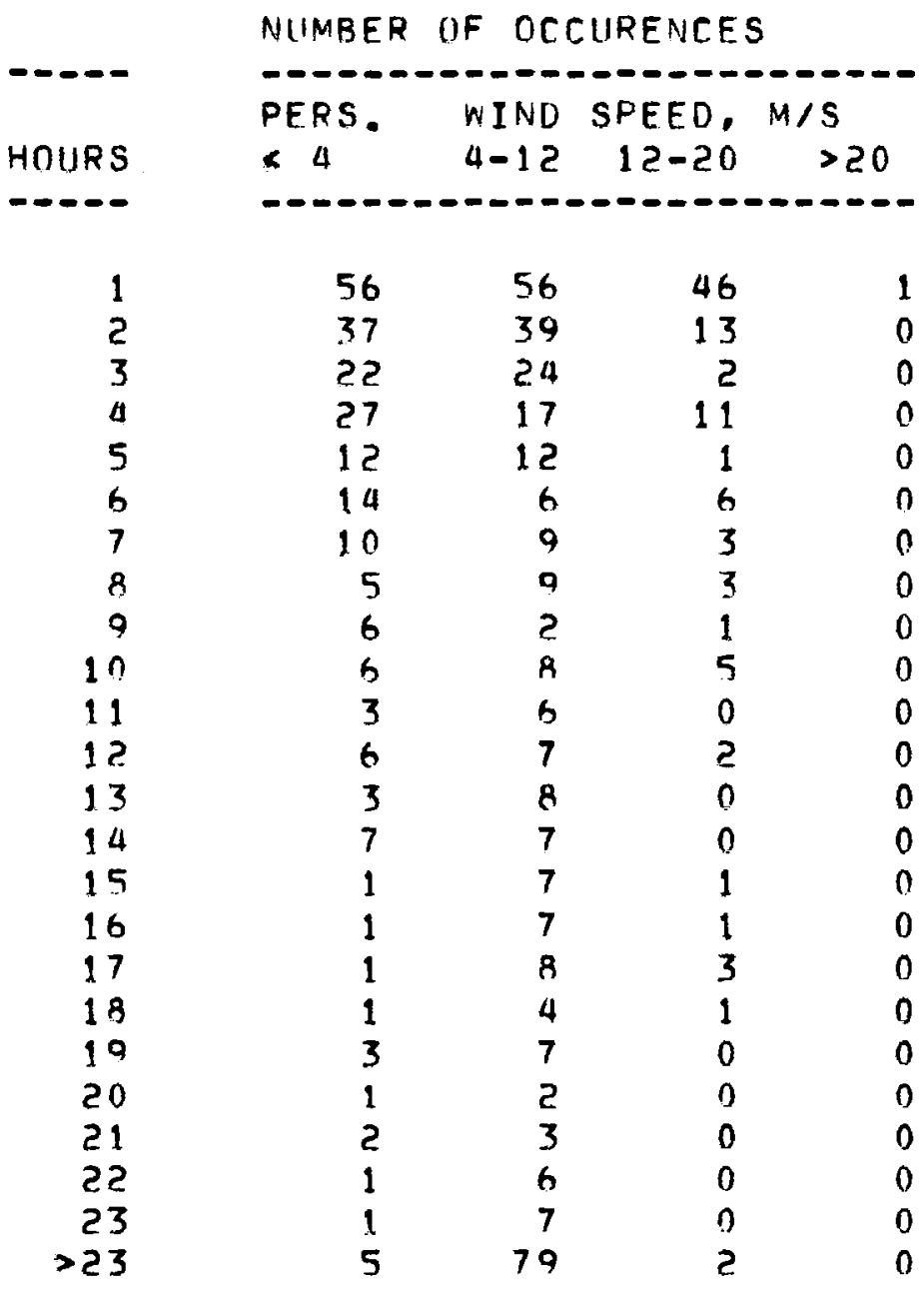




SITE IO:
SITE LOCATTIN: RLOCK ISLAND, RT.
DATA :
JANIIARY 1979 THROUGH DECEM

WIND DIR

(ELEV A)

ALPHA ALPHA ALPHA ALPHA

$(A, B)(B, C)(A, C)(A, P, C) \quad \% A \quad \% B \quad \% C$

$\begin{array}{lrrrrrrr}\text { N } & 1.41 & -0.22 & 0.20 & 0.11 & 4.98 & 3.29 & 4.07 \\ \text { NNE } & 0.16 & 0.27 & 0.24 & 0.24 & 4.95 & 4.86 & 1.10 \\ \text { NE } & 0.17 & 0.31 & 0.27 & 0.28 & 5.00 & 6.10 & 4.98 \\ \text { ENE } & 0.31 & 0.14 & 0.18 & 0.17 & 3.15 & 2.94 & 4.14 \\ \text { E } & 1.87 & -0.21 & 0.33 & 0.21 & 3.70 & 1.90 & 2.92 \\ \text { ESE } & 1.28 & -0.16 & 0.21 & 0.13 & 3.16 & 2.15 & 3.59 \\ \text { SE } & 1.06 & -0.01 & 0.27 & 0.21 & 3.31 & 3.12 & 4.51 \\ \text { SSF } & 0.40 & 0.19 & 0.25 & 0.23 & 4.69 & 5.34 & 4.22 \\ \text { S } & 0.59 & 0.18 & 0.29 & 0.27 & 6.23 & 8.13 & 7.29 \\ \text { SSN } & 0.49 & 0.25 & 0.31 & 0.30 & 5.97 & 11.69 & 9.80 \\ \text { SW } & 0.50 & 0.26 & 0.32 & 0.31 & 11.23 & 13.37 & 10.72 \\ \text { WSW } & 0.59 & 0.24 & 0.33 & 0.31 & 6.86 & 8.65 & 8.57 \\ \text { W } & 0.34 & 0.25 & 0.27 & 0.27 & 5.72 & 5.81 & 7.13 \\ \text { WNW } & 0.35 & 0.25 & 0.27 & 0.27 & 6.34 & 6.48 & 6.91 \\ \text { NW } & 0.27 & 0.15 & 0.19 & 0.18 & 8.36 & 8.38 & 8.57 \\ \text { NN.N } & 0.82 & -0.06 & 0.17 & 0.12 & 5.94 & 4.47 & 7.39\end{array}$

NOTES:

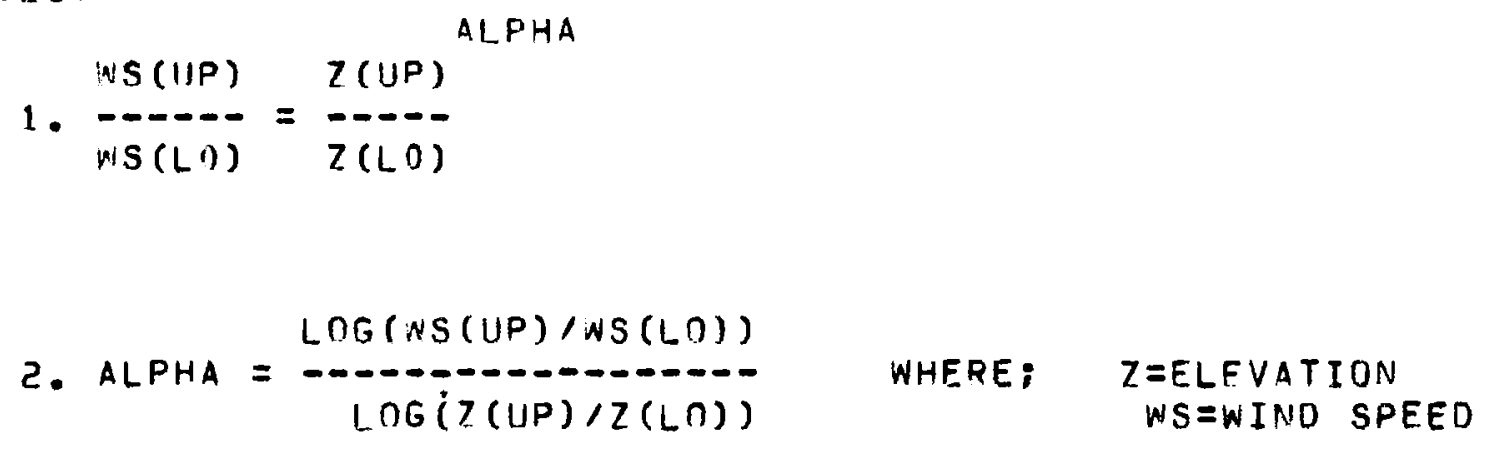




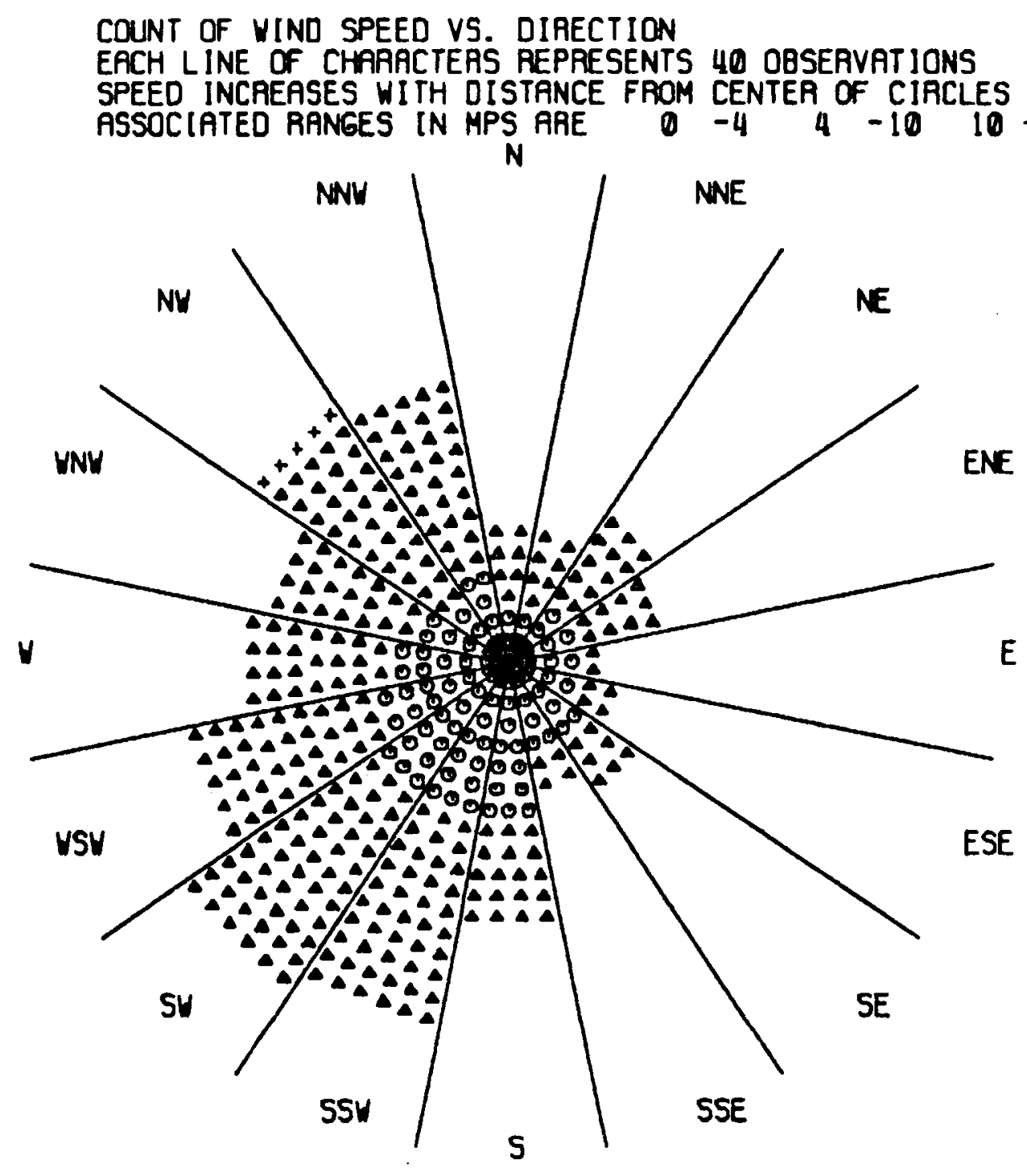

FIGURE 1. (RI) Wind Rose for Lowest Sensor Level 


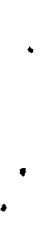

: 
BOARDMAN, OREGON 


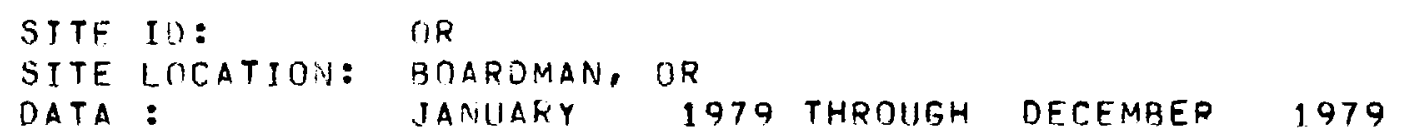

(A) 70.1

(B) 39.6

(C) 9.1

$\begin{array}{ll}5.4 & 250.8 \\ 4.8 & 241.2 \\ 3.8 & 237.7\end{array}$

3.9

3.5

2.7

\begin{tabular}{|c|c|c|c|c|c|c|}
\hline \multirow{2}{*}{\multicolumn{2}{|c|}{$\begin{array}{l}\text { ELEVATIDN } \\
\text { (METERS) }\end{array}$}} & $\begin{array}{r}\text { WIND } \\
\text { SPEED }\end{array}$ & $\begin{array}{l}\text { WIND } \\
\text { DIR. }\end{array}$ & DATE & TIME & \multirow{2}{*}{ OTHER LEVELS } \\
\hline & & ---- & $----\infty$ & $---\infty-\infty-\infty$ & $--\infty-\infty$ & \\
\hline (A) & $7 n .1$ & 27.7 & 249.0 & $07 / 28 / 79$ & $00: 00$ & $\begin{array}{l}\text { (B) }-999.9 \\
(C)-999.9\end{array}$ \\
\hline (B) & 39.6 & 39.8 & 271.0 & $07 / 01 / 79$ & $13: 00$ & $\begin{array}{l}\text { (A) }-999.9 \\
\text { (C) } 34.9\end{array}$ \\
\hline$(C)$ & 9.1 & 34.9 & 275.0 & $07 / 01 / 79$ & $13: 00$ & $\begin{array}{l}\text { (A) }-999.9 \\
\text { (B) } 39.8\end{array}$ \\
\hline
\end{tabular}


SITE IN: $\quad$ NR

STTE LOCATION: GOARDMAN, OR

DATA : JANUARY 1979 THROUJG DECFMEER 1979

1.4 NINI SPEED AND DIRECTION VERSUS TIME-OF-DAY

TIME

OF DAY

LEVEL A

$-0-\infty$

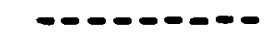

WS

WD

LEVEL B

LEVEL C

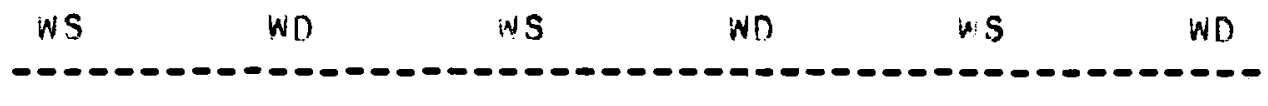

$00: 00$

5.7

231.3

5.0

219.3

3.6

213.3

$5.6 \quad 233.1$

5.0

221.4

3.6

214.0

$02: 00$

5.6

233.0

4.9

219.4

214.5

ก 3:00

5.5

4.8

3.5

214.0

1) $4: 00$

$5.4 \quad 235.5$

$05: 00$

$5.2 \quad 237.2$

$06: 00$

$5.0 \quad 241.8$

4.6

3.4

$3.4 \quad 215.3$

$4.6 \quad 225.11$

$4.5 \quad 235.3$

$3.4 \quad 222.3$

$07: 00$

$4.8 \quad 254.8$

4.7 264.3

4.5

$3.6 \quad 233.4$

$3.7 \quad 248.3$

$08=00$

4.7

$4.4 \quad 259.5$

$3.7 \quad 262.9$

$09: 00$

272.5

$10: 00$

4.9

281.6

4.3

3.7

3.9

274.1

$4.5 \quad 276.3$

282.0

$4.8 \quad 289.6$

$4.4 \quad 288.1$

3.9

290.1

$12: 00$

292.6

$4.9 \quad 291.6$

$4.5 \quad 291.4$

$13: 00$

590.4

4.7

3.9

291.4

$4.7 \quad 299.1$

294.4

5.0

296.2

$15: 00$

5.3

4.8

4.1

293.8

$5.3 \quad 278.8$

$17: 00$

5.6

258.0

$5.9 \quad 241.0$

$18: 00$

$19: 00$

232.7

6.3

234.7

294.

4.0

4.0

295.6

$4.9 \quad 277.9$

$5.0 \quad 252.0$

3.9

278.5

$3.8 \quad 247.0$

$5.2 \quad 234.0$

3.8

228.9

5.4224 .2

3.9

221.9

$5.5 \quad 225.6$

$4.0 \quad 223.0$

$6.3 \quad 235.6$

$5.5 \quad 226.2$

$4.0 \quad 223.8$

$6.0 \quad 237.8$

$5.3 \quad 227.2$

$3.9 \quad 223.7$

$22: 00$
$23: 00$

$5.8 \quad 234.6$

5.1221 .3

$3.7 \quad 216.7$ 
$\begin{array}{ll}\text { SITE ID: } & \text { OR } \\ \text { SITE LOCATION: } & \text { BOARUMAN, OR } \\ \text { OATA : } & \text { JAMUARY }\end{array}$

OATA:

1979 THROUJH DECFMRER

1979

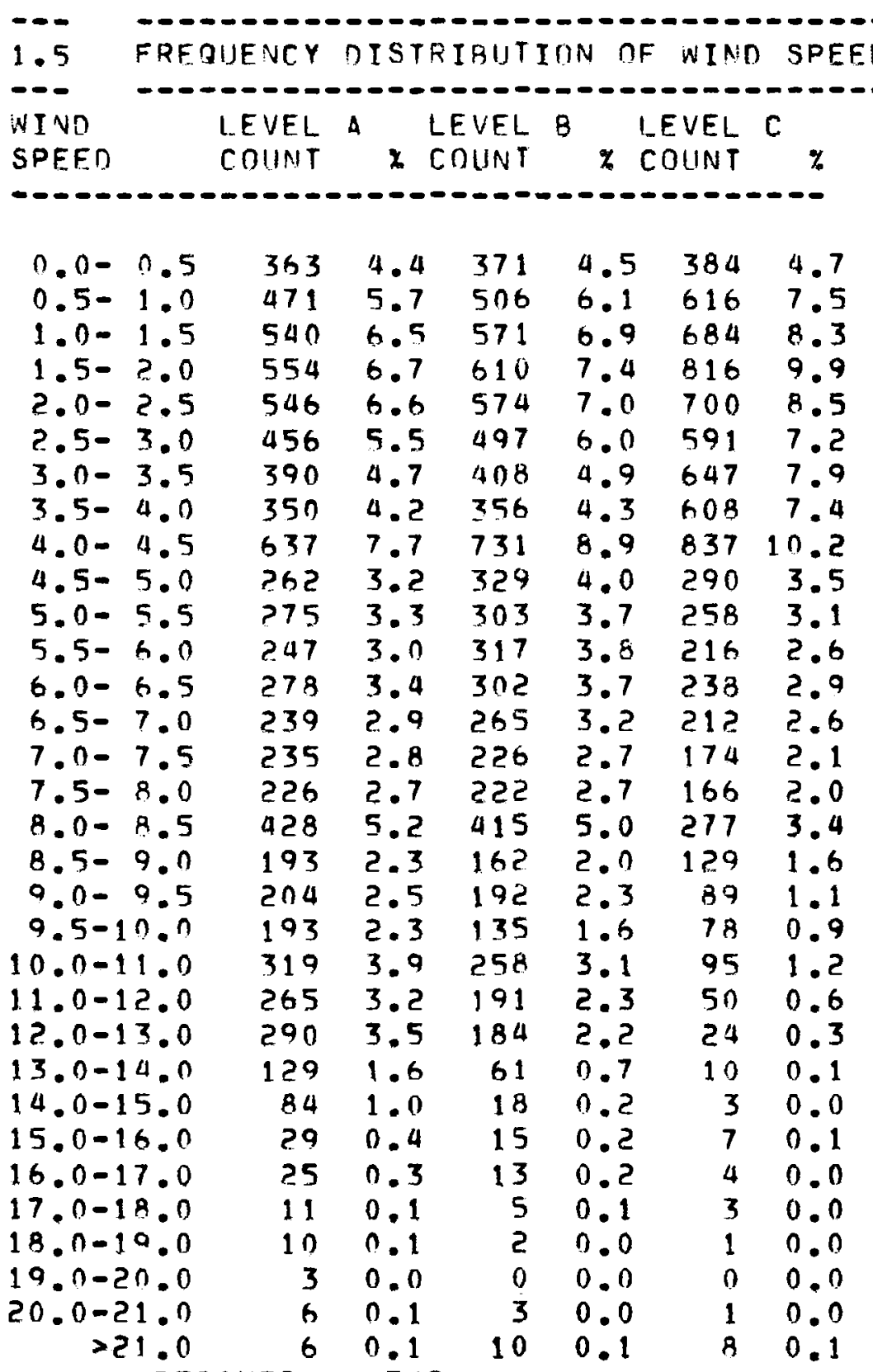

RECNVERY RATES

LEVEL A LEVEL B LEVEL C

$$
94.3 \quad 04 . ? \quad 93.8
$$




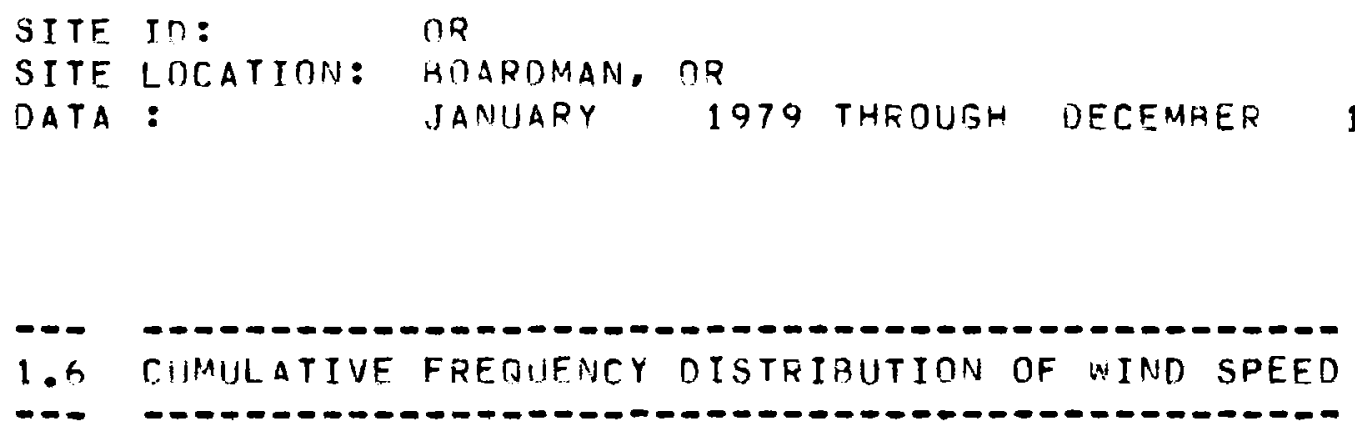

LEVEL A

$---0$

W IND

SPEED

$---$

0.5

1.0

1.5

2. 0

2. 5

3. 0

3.5

4.0

4.5

5.0

5.5

6.0

6.5

7.0

7.5

8.0

8. 5

9.0

9. 5

10.0

11.0

12.0

13.0

14.0

15.0

16.0

17.0

18.0

19.0

20.0

21.0

$>21.0$

363

834

1374

1928

2474

2930

3320

3670

$43 \cap 7$

4569

4844

5091

5369

4608

5843

6069

6497

6690

6894

7087

7406

7671

7961

8090

R174

8203

8ट2 8

8239

8249

หว5?

82 58

8264
LEVEL $B$

$\begin{array}{cc}\text { CFD } & \text { CFD } \\ \text { (ARS) } & (\%) \\ ---\infty & -----\end{array}$

4. 39

10.09

15.63

23.33

29.94

35.45

40.17

44.41

5?. 12

55.29

53.62

61.60

64.97

67.86

70.70

73.44

78.62

80.95

83.42

85.76

89.62

92.82

96.33

97.89

98.91

99.26

99.56

99.70

99.82

99.85

99.93

100.00
$---------D-0$

CFD CFD

(ABS) (\%)

$----0--$

3714.50

$877 \quad 10.63$

$1448 \quad 17.55$

$2058 \quad 24.94$

$2632 \quad 31.90$

$3129 \quad 37.92$

353742.86

$3893 \quad 47.18$

462456.03

495360.02

$5256 \quad 63.69$

$5573 \quad 67.54$

587571.19

$6140 \quad 74.41$

636677.14

$6588 \quad 79.84$

700384.86

$7165 \quad 86.83$

7357

7492

7750

7941

8125

8186

8204

8219

8232

8237

ค2 39

8239

8242

8252
89.15

0.79

93.92

96.23

98.46

$99 . ? 0$

99.42

99.60

99.76

$99.8 ?$

99.84

99.84

99.88

100.00
LEVEL C

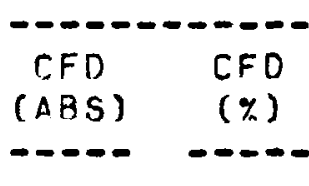

$384 \quad 4.67$

$1000 \quad 12.17$

168420.50

$2500 \quad 30.43$

$3200 \quad 38.95$

379146.14

443854.02

$5046 \quad 61.42$

588371.60

617375.13

$6431 \quad 78.27$

$6647 \quad 9.0 .90$

$688583.8 n$

$7097 \quad 86.38$

$7271 \quad 88.50$

$7437 \quad 90.52$

771493.89

784395.46

$7932 \quad 96.54$

$8010 \quad 07.49$

$8105 \quad 98.65$

815599.26

817999.55

$8189 \quad 99.67$

$8192 \quad 99.71$

819909.79

$82 \cap 399.84$

$9206 \quad 99.88$

$8207 \quad 99.89$

$8207 \quad 99.89$

$8208 \quad 09.90$

9216 100.00 
SITE ID: OR

SITE LOCATIDN: GOARDMAN, OR

DATA JANIIARY 197

1979 THROUGH DECEMBER 1979

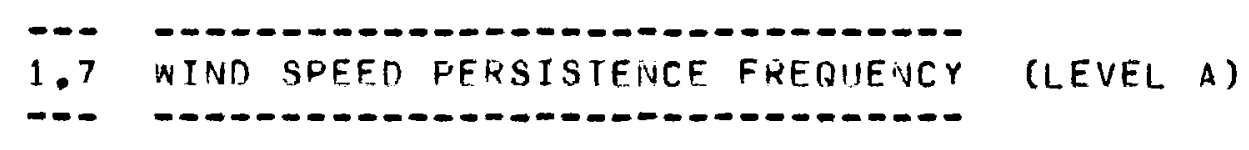

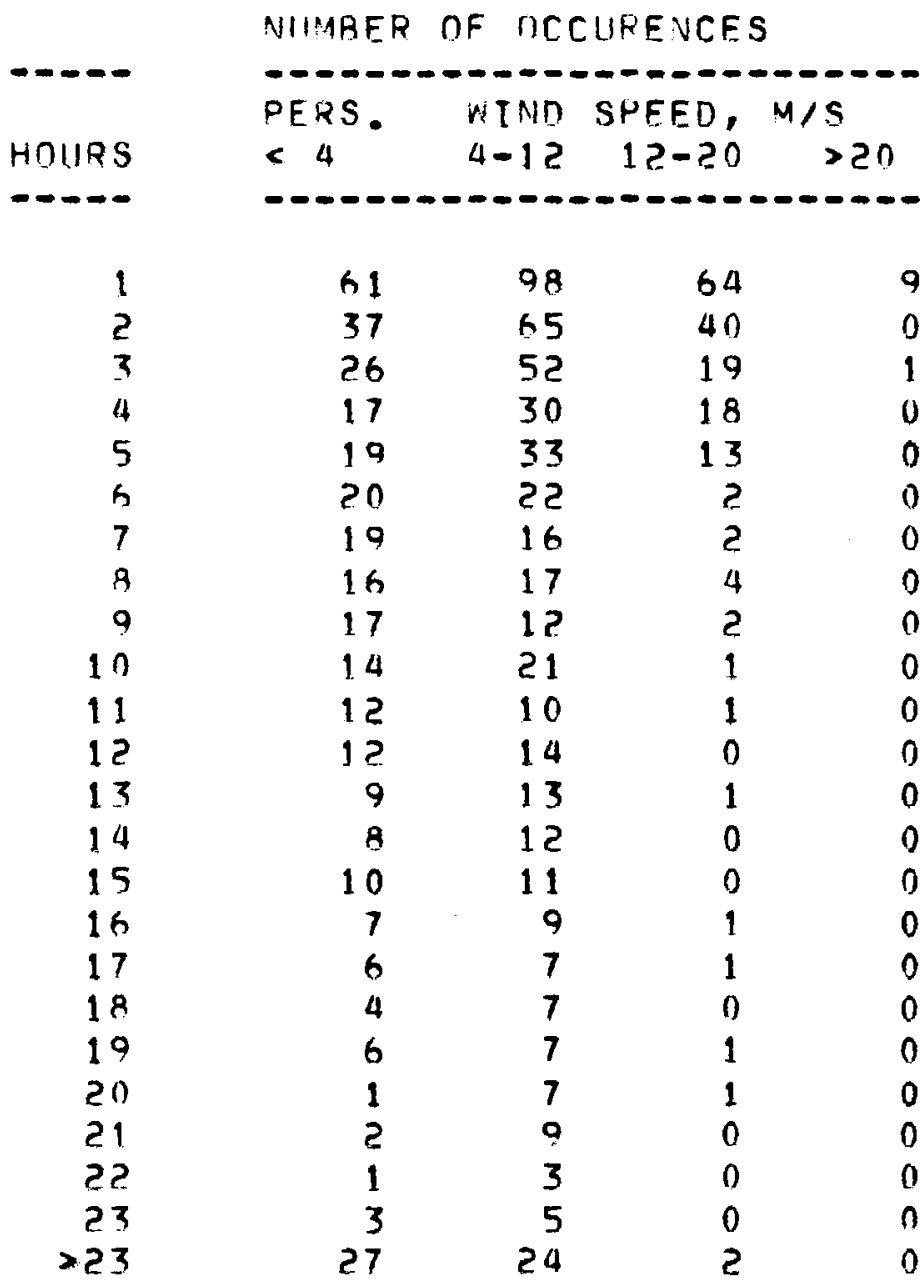




\begin{tabular}{|c|c|c|c|c|c|}
\hline SITE & In: & MR & & & \\
\hline SITE & LOCATTON: & ROARחMAN, & $\cap R$ & & \\
\hline DATA & $:$ & JANUARY & 1979 & THROIIGH & DECE \\
\hline
\end{tabular}

WIND DIR

(FLEVA)

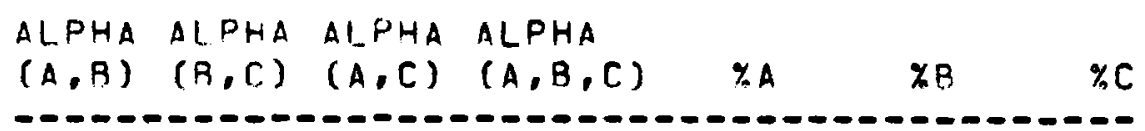

$\begin{array}{lrrrrrrr}\text { N } & 0.31 & 0.08 & 0.15 & 0.14 & 2.86 & 2.48 & 3.14 \\ \text { NNE } & 0.06 & 0.09 & 0.08 & 0.09 & 4.11 & 3.67 & 3.26 \\ \text { NE } & 0.26 & 0.09 & 0.14 & 0.13 & 5.86 & 5.82 & 4.08 \\ \text { ENE } & 0.39 & 0.02 & 0.12 & 0.10 & 5.28 & 5.32 & 3.97 \\ \text { E } & 0.30 & 0.08 & 0.14 & 0.13 & 4.25 & 3.62 & 2.92 \\ \text { ESE } & 0.12 & 0.17 & 0.15 & 0.15 & 2.43 & 3.05 & 1.94 \\ \text { SE } & 0.01 & 0.17 & 0.12 & 0.13 & 1.80 & 2.00 & 1.69 \\ \text { SSE } & -0.01 & 0.15 & 0.10 & 0.11 & 1.16 & 1.37 & 1.63 \\ \text { S } & 0.15 & 0.24 & 0.21 & 0.22 & 2.13 & 3.14 & 5.55 \\ \text { SSW } & -0.04 & 0.23 & 0.15 & 0.17 & 4.84 & 10.14 & 16.47 \\ \text { SW } & 0.19 & 0.20 & 0.20 & 0.20 & 16.23 & 16.15 & 12.43 \\ \text { WSW } & 0.14 & 0.17 & 0.16 & 0.17 & 27.98 & 24.19 & 20.81 \\ \text { N } & 0.06 & 0.07 & 0.06 & 0.06 & 12.16 & 10.77 & 12.57 \\ \text { WNW } & -0.01 & 0.07 & 0.05 & 0.05 & 3.91 & 3.70 & 4.64 \\ \text { NW } & -0.09 & 0.05 & 0.01 & 0.02 & 2.70 & 2.47 & 2.62 \\ \text { NNW } & -0.17 & 0.15 & 0.06 & 0.08 & 2.23 & 1.98 & 2.22\end{array}$

NOTES:

$$
\begin{aligned}
& \text { NS(UP) } \\
& \text { WS(LO) } \\
& \text { WS(UP) }
\end{aligned}
$$

ALPHA

$$
\text { 2. ALPHA }=\frac{\operatorname{LOG}(W S(U P) / N S(L O))}{\operatorname{LOG}(\angle(U P) / Z(L O))} \quad \text { HHERE: } \quad \text { ZEELEVATION }
$$


COUNT OF YINO SPEED VS. DIRECTION

EACH LINE OF CHARACTERS REPRESENTS 40 OBSERVATIONS

SPEED INCRERSES WITH DISTRNCE FROA CENTER OF CIRCLES

RSSOC IATED RANGES IN MPS ARE $0-4 \quad 4 \quad-10 \quad 10-20 \quad 20-$

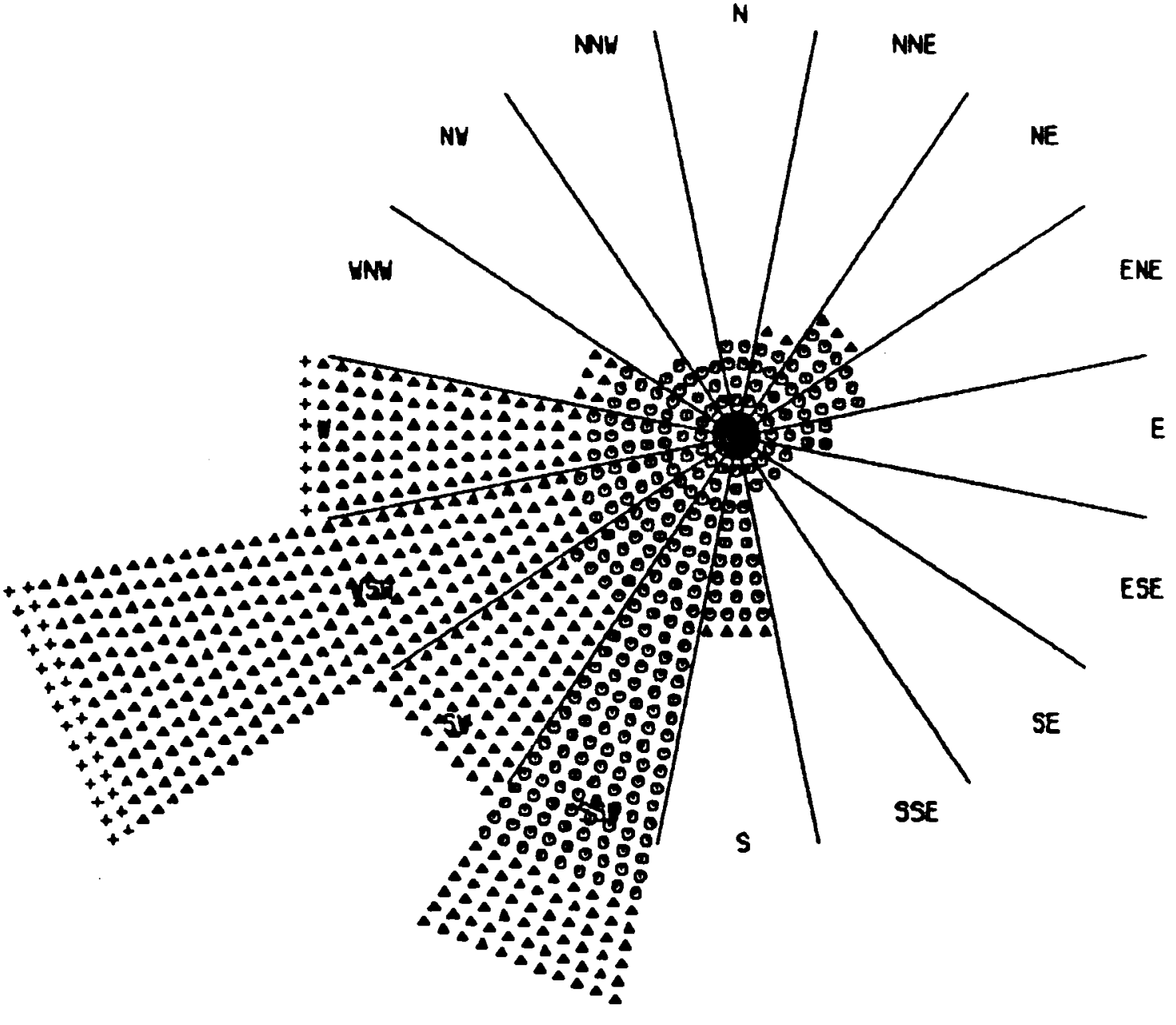

FIGURE 1. (OR) Wind Rose for Lowest Sensor Level 
BOONE, NORTH CAROLINA 


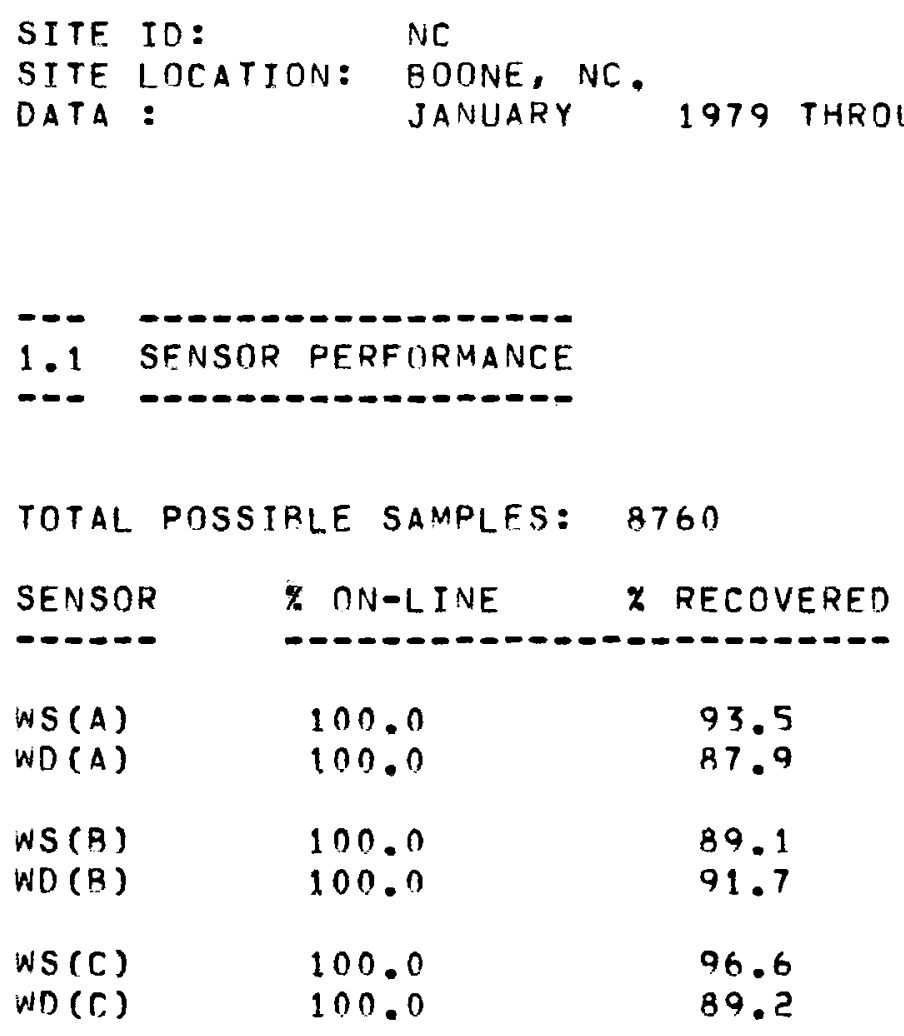

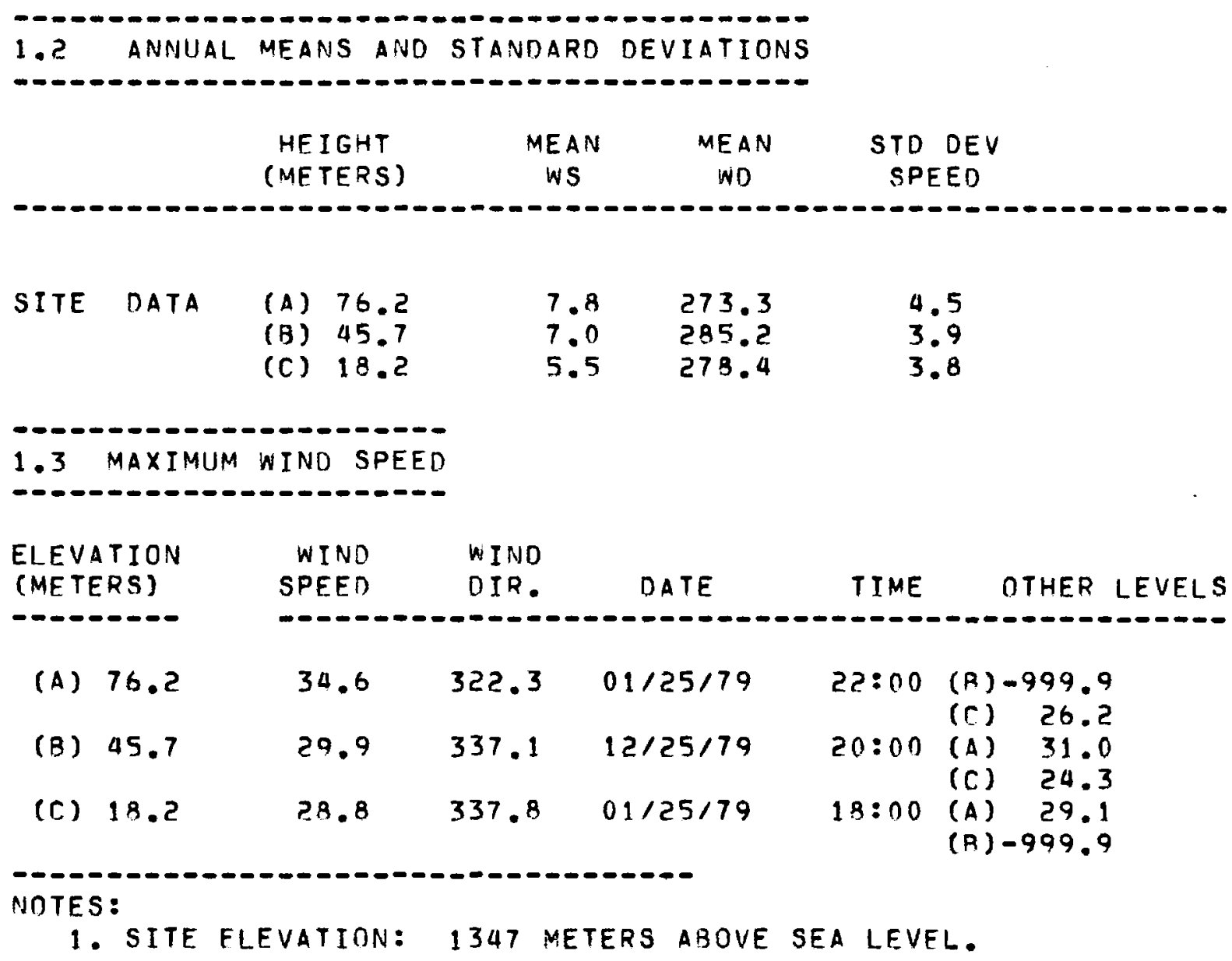


SITE ID: $\quad$ NC

SITE LOCATION: BOONE, NC.

DATA : JANIJARY 1979 THROUGH DECEMPER 1979

1.4 WIND SPEED ANO DIRECTION VERSIIS TIME-OF-DAY

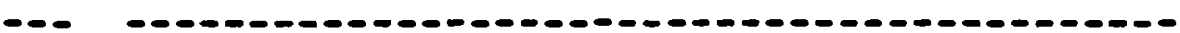

TIME

OF DAY

OF- D.-

$00: 00$

$01: 00$

$0 ?: 00$

$03: 00$

$04: 00$

05:00

$06: 00$

$07: 00$

$08: 00$

0 $9: 00$

$10: 00$

$11: 00$

$12: 00$

$13: 00$

14:00

$15: 00$

16:00

$17=00$

$18: 00$

$19: 00$

20:00

र $1=00$

$22: 00$

$23: 00$
LEVEL A

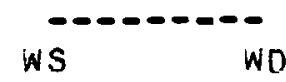

$8.5 \quad 263.7$

$8.5 \quad 266.6$

$8.6 \quad 270.7$

$8.5 \quad 271.4$

$8.6 \quad 274.5$

$8.6 \quad 279.1$

$8.4 \quad 281.3$

$8.2 \quad 283.9$

$7.8 \quad 287.0$

$7.4 \quad 285.5$

$7.2 \quad 279.4$

$7.2 \quad 275.7$

$7.0 \quad 273.2$

$6.9 \quad 269.0$

$6.9 \quad 265.5$

$7.1 \quad 266.1$

$7.0 \quad 269.1$

$7.1 \quad 272.0$

$7.4 \quad 269.4$

$7.6 \quad 269.1$

$8.0 \quad 273.5$

8. 1

8.2

8.3
270.1

260.4

266.3
LEVEL B

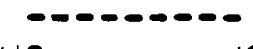

WS

WD

WS

LEVEL C

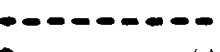

$5.9 \quad 273.9$

$5.9 \quad 275.7$

$6.0 \quad 278.9$

6.0 280.2

$6.1 \quad 283.7$

$6.2 \quad 286.7$

$6.0 \quad 289.0$

$5.7 \quad 292.1$

$5.5 \quad 291.9$

$5.3 \quad 285.1$

$5.1 \quad 278.7$

$5.1 \quad 276.6$

4.9273 .8

$4.8 \quad 270.2$

$4.8 \quad 266.0$

$4.9 \quad 267.3$

$4.9 \quad 270.6$

4.9271 .9

$5.1 \quad 274.2$

$5.3 \quad 279.4$

$5.6 \quad 281.8$

$5.7 \quad 276.8$

$5.7 \quad 278.4$

$5.8 \quad 274.7$ 
SITE ID: NC

SITE LOCATION: BOONE, NC.

JANUARY

1979 THROUGH DECEMBER

1979

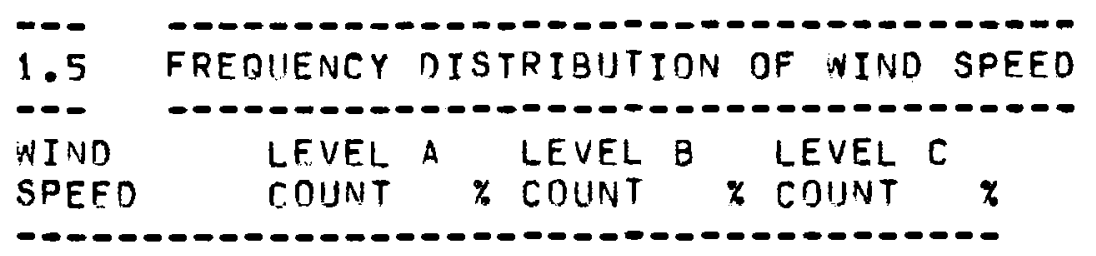

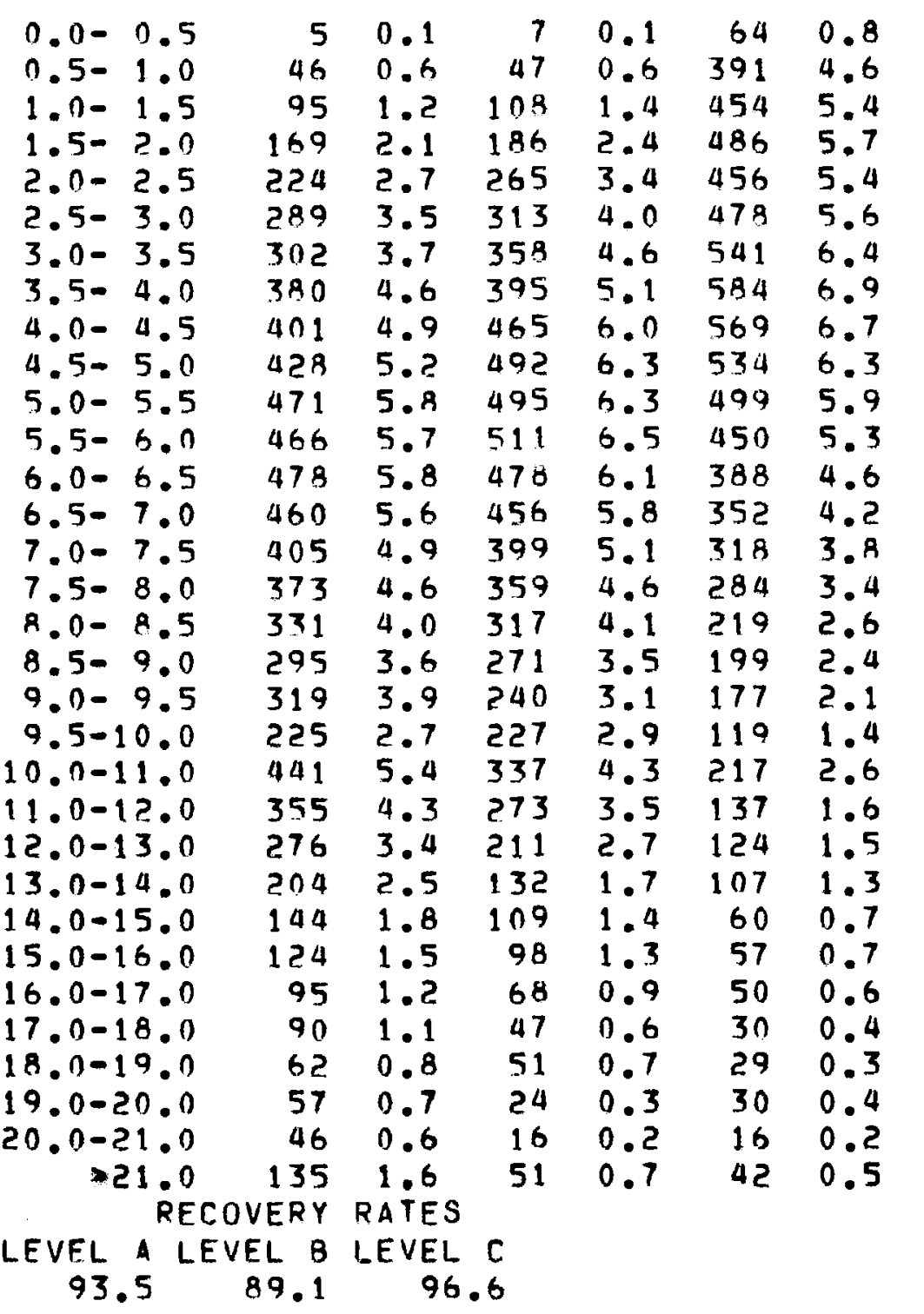


SITE ID: NC

SITE LOCATION: BOONE, NC.

DATA

JANUARY

1979 THROUSH DECEMBER

1979

1.6

CIIMIILATIVE FREDUENCY DISTRIBIITION OF WIND SPEED

$-\infty$

LEVEL A

WIND

SPEED

$---O-$

0.5

1.0

1.5

2.0

2.5

3.0

3.5

4.0

4.5

5.0

5.5

6.0

6.5

7.0

7.5

8.0

8.5

9.0

9.5

10.0

11.0

12.0

13.0

14.0

15.0

16.0

17.0

18.0

19.0

20.0

21.0

$>21.0$

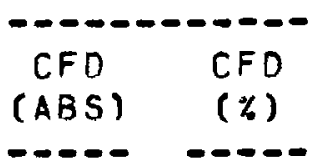

50.06

$51 \quad 0.62$

$146 \quad 1.78$

3153.85

$539 \quad 6.58$

$828 \quad 10.11$

$1130 \quad 13.80$

$1510 \quad 18.43$

$1911 \quad 23.33$

$2339 \quad 28.56$

$2810 \quad 34.31$

$3276 \quad 40.00$

$3754 \quad 45.83$

4?14 51.45

$4619 \quad 56.39$

$4992 \quad 60.94$

$5323 \quad 64.99$

$561 R \quad 68.59$

593772.48

616275.23

660380.61

$6958 \quad 84.95$

$7234 \quad 88.32$

$7438 \quad 90.81$

$7582 \quad 92.57$

$7706 \quad 94.08$

780195.24

7891

7953

96.34

8010

07.09

97.79

8056

98.35

8191100.00
LEVEL B

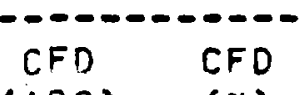

( $\triangle 8 S$ )

---.-

$7 \quad 0.09$

$54 \quad 0.69$

$16 ? 2.03$

$348 \quad 4.46$

6137.85

$926 \quad 11.86$

$1294 \quad 16.45$

167921.51

$2144 \quad 27.47$

$2636 \quad 33.77$

313140.11

$3642 \quad 46.66$

$4120 \quad 52.78$

$4576 \quad 58.62$

$4975 \quad 63.73$

$5334 \quad 68.33$

$\begin{array}{ll}5651 & 72.39\end{array}$

59?? 75.36

616278.94

6389

6726

6999

7210

7342

7451

7549

7617

7664

7715

7730

7755

7806
LEVEL C

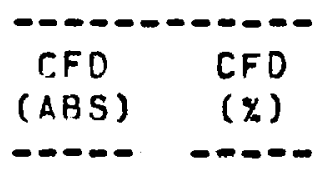

640.76

$455 \quad 5.38$

$909 \quad 10.74$

$1395 \quad 16.49$

185121.88

$2329 \quad 27.53$

$2870 \quad 33.92$

$3454 \quad 40.82$

$4023 \quad 47.55$

$4557 \quad 53.86$

$5056 \quad 59.76$

$5506 \quad 65.08$

$5894 \quad 69.66$

$6246 \quad 73.82$

656477.59

$6848 \quad 80.94$

$\begin{array}{ll}7067 & 83.52\end{array}$

$\begin{array}{ll}7266 & 85.88\end{array}$

$7443 \quad 87.97$

$7562 \quad 89.37$

$7779 \quad 91.94$

$7916 \quad 93.56$

$8040 \quad 05.02$

$8147 \quad 96.29$

820797.00

826497.67

831498.26

834498.62

837398.96

$9403 \quad 99.31$

841999.50

8461100.00 
SITE ID: $\quad$ MC

SITE LOCATION: HOONE, NC.

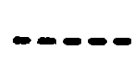

HOURS

$---\infty$

?

3

4

5

6

7

8

9

10

11

12

13

14

15

16

17

18

19

20

21

2?

23

.23
NUMBER OF OCCURENCES
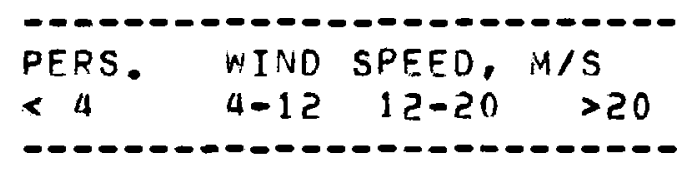

$\begin{array}{rrrr}100 & 121 & 91 & 13 \\ 53 & 71 & 45 & 9 \\ 42 & 41 & 27 & 4 \\ 28 & 22 & 26 & 3 \\ 20 & 30 & 18 & 1 \\ 17 & 19 & 7 & 1 \\ 11 & 21 & 10 & 1 \\ 8 & 19 & 6 & 2 \\ 5 & 26 & 3 & 1 \\ 4 & 19 & 6 & 1 \\ 7 & 16 & 8 & 0 \\ 2 & 11 & 4 & 1 \\ 5 & 7 & 2 & 1 \\ 4 & 11 & 1 & 0 \\ 8 & 14 & 1 & 1 \\ 0 & 8 & 2 & 1 \\ 4 & 8 & 0 & 1 \\ 1 & 7 & 2 & 0 \\ 1 & 6 & 1 & 0 \\ 1 & 6 & 0 & 0 \\ 1 & 6 & 0 & 0 \\ 2 & 5 & 0 & 0 \\ 0 & 2 & 1 & 0 \\ 3 & 62 & 1 & 0\end{array}$




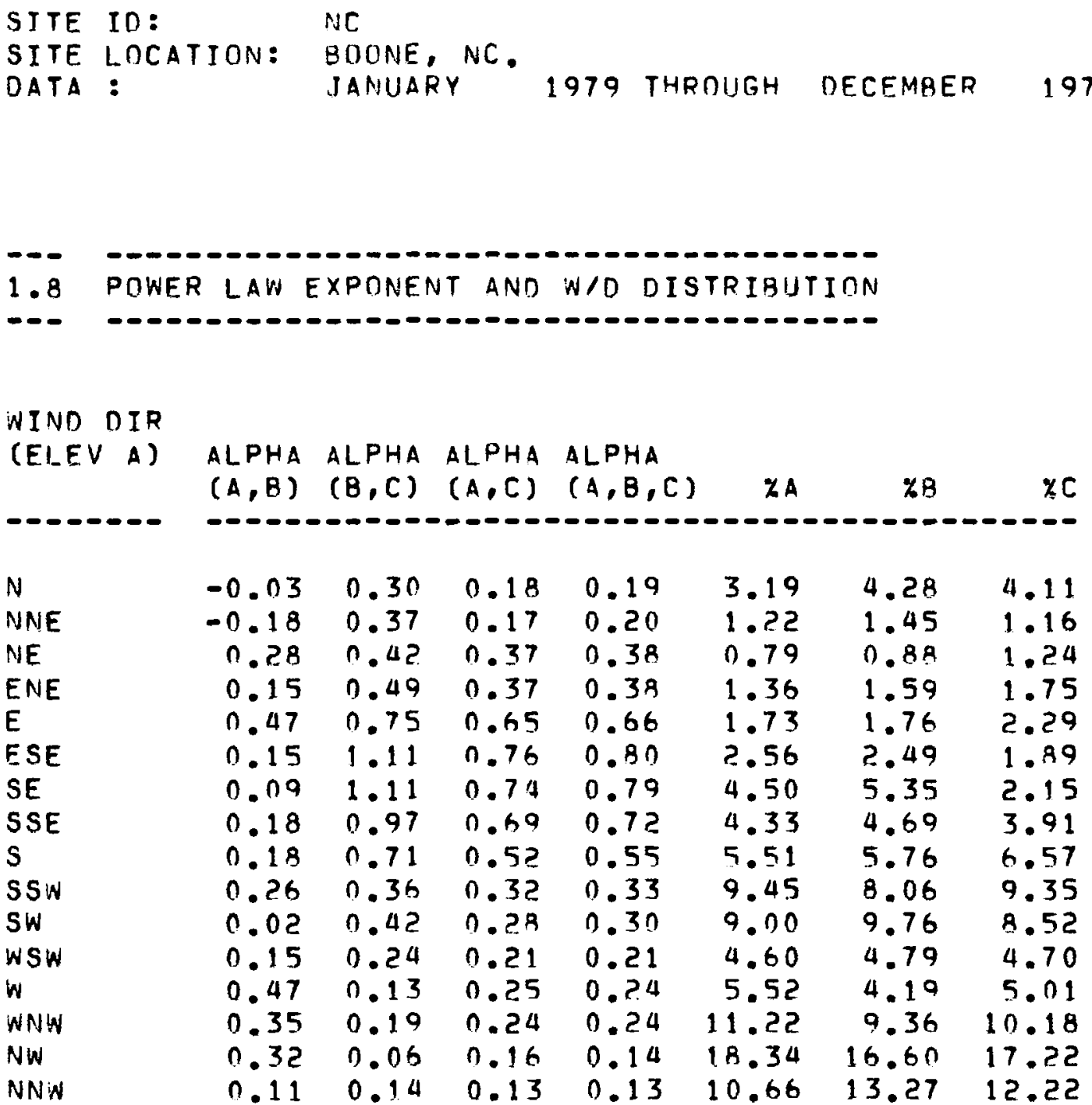

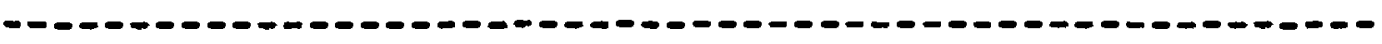
NOTES:

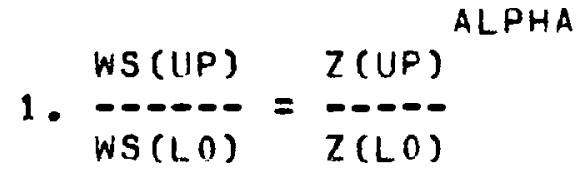

$$
\begin{aligned}
& \begin{array}{l}
\text { 2. ALPHA }=\frac{\operatorname{LOG}(W S(U P) / W S(L O))}{\operatorname{LOG}(Z(U P) / Z(L O))} \quad \text { WHERE; } \quad \text { W=ELEVATION } \\
\text { WS=WIND SPEED }
\end{array}
\end{aligned}
$$


++ COUNT OF WIND SPEED VS. DIRECTION

++++ EACH LINE OF CHARACTERS REPRESENTS 40 OBSERVATIONS

+++++ SPEED INCREASES $V I$ IT DISTRNCE FROM CENTER OF CIRCLES

++++++5 SOCIRTED RPNGES IN MPS RRE $00-4 \quad 4-10 \quad 10-20 \quad 20-$

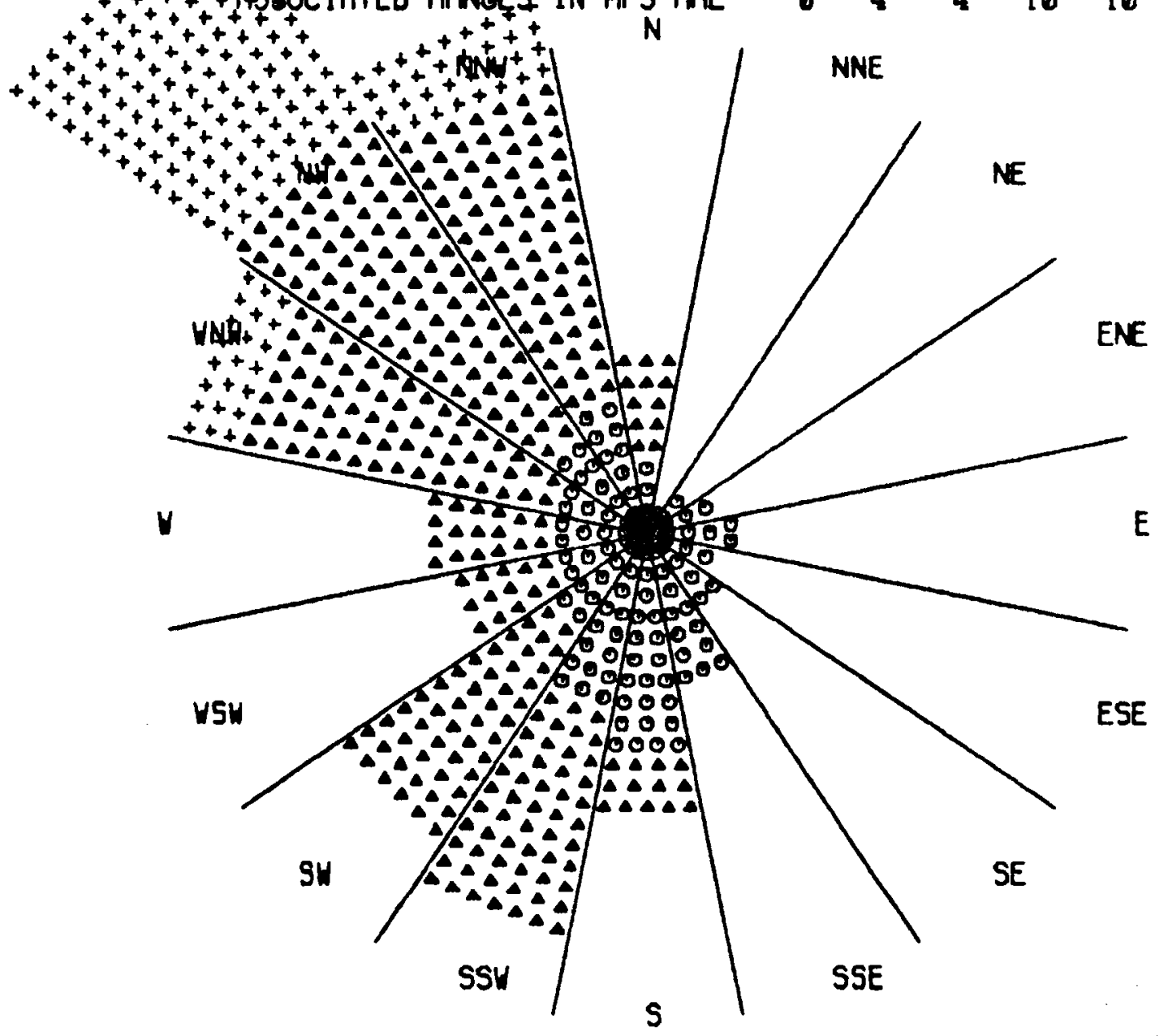

FIGURE 1. (NC) Wind Rose for Lowest Sensor Level 
.

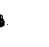

- 
CLAYTON, NEW MEXICO 


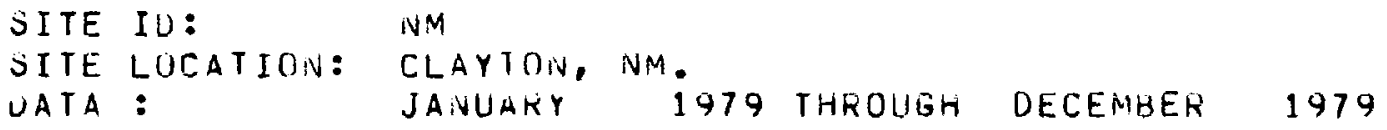
(A) 45.7
27
$-999$.
$10 / 30 / 79$
$18: 00$
(B)
25.0
(B) 30.0
25.0
317.1
$10 / 30 / 79$
$18: 00$
(A)
21. 1
(C) 9.1
$21 \cdot 3$
298.6
$03 / 22 / 79$
$11: 00$
(C) 21.1
(A) 25.2
(B) 24.4

NOTES:

1. Site elevation: 1536 METERS abOVE SEA LEVEL. 
SITE IO:

SITE LOCATION: CLAYTUN, NM.

UATA : JANUARY 1979 THROUGH DECENBEF 1979

1.4 WIND SPEED AND UIRECTION VERSUS IIME-OF-UAY

\begin{tabular}{|c|c|c|c|c|c|c|}
\hline TIME & & $\begin{array}{ll}E & A \\
--\infty & --\end{array}$ & & $\begin{array}{l}1 \\
B \\
---\end{array}$ & & $\begin{array}{l}L \\
L\end{array}$ \\
\hline UF DAY & wS & WD & WS & Wo & WS & wO \\
\hline------ & - & -0 & & & & $\ldots$ \\
\hline $00: 00$ & 6.9 & 230.0 & 6.0 & 239.7 & 4.2 & 240.7 \\
\hline $01: 00$ & 6.9 & 238.1 & 6.0 & 247.3 & 4.2 & 247.3 \\
\hline $02: 00$ & 7.1 & 245.1 & 6.1 & 252.7 & 4.5 & 251.0 \\
\hline $03: 00$ & 7.1 & 249.2 & 6.2 & 255.9 & 4.3 & 251.7 \\
\hline $04: 00$ & 7.1 & 253.4 & 6.2 & 259.0 & 4.3 & 253.9 \\
\hline $05: 00$ & 7.0 & 259.5 & 6.1 & 263.4 & 4.2 & 257.0 \\
\hline $06: 00$ & 6.9 & 261.0 & 6.0 & 266.2 & 4.3 & 259.2 \\
\hline $07: 00$ & 6.8 & 261.4 & 6.1 & 264.3 & 4.9 & 255.4 \\
\hline $08: 00$ & 7.1 & 256.8 & 6.7 & 259.9 & 5.8 & 252.2 \\
\hline $09: 00$ & 7.4 & 239.8 & 7.2 & 243.3 & 6.4 & 239.1 \\
\hline $10: 00$ & 7.5 & 219.1 & 7.3 & 224.7 & 6.5 & 223.1 \\
\hline $11: 00$ & 7.6 & 192.7 & 7.4 & 199.6 & 6.0 & 198.1 \\
\hline $12: 00$ & 7.6 & 177.6 & 7.4 & 184.2 & 6.6 & 182.4 \\
\hline $13: 00$ & 7.8 & 174.5 & 7.6 & 181.9 & 6.8 & 178.9 \\
\hline $14: 00$ & 7.9 & 170.9 & 7.7 & 176.7 & 6.8 & 173.4 \\
\hline $15: 00$ & 7.8 & 165.6 & 7.6 & 171.9 & 6.6 & 167.6 \\
\hline $16: 00$ & 7.8 & 161.6 & 7.4 & 167.3 & 6.3 & 163.0 \\
\hline $17: 00$ & 7.4 & 153.5 & 7.0 & 158.6 & 5.7 & 153.5 \\
\hline $18: 00$ & 7.3 & 151.1 & 6.6 & 157.1 & 5.2 & 154.2 \\
\hline $19: 00$ & 7.3 & 157.1 & 6.5 & 150.1 & 4.8 & 166.0 \\
\hline $20: 00$ & 7.3 & 174.8 & 6.4 & 186.0 & 4.5 & 185.4 \\
\hline $21: 00$ & 7.2 & 185.2 & 6.3 & 197.7 & 4.5 & 203.8 \\
\hline $22: 00$ & 7.1 & 204.3 & 6.2 & 218.6 & 4.3 & 224.4 \\
\hline $23: 00$ & 7.1 & 218.9 & 6.1 & 229.0 & 4.3 & 232.7 \\
\hline
\end{tabular}


$\begin{array}{ll}\text { SITE IO: } & \text { WM } \\ \text { SITE LUCATION: } & \text { CLAYIUN, NM. } \\ \text { UATA : } & \text { JANUARY }\end{array}$

1979 THROUGH DECEMEER

1979

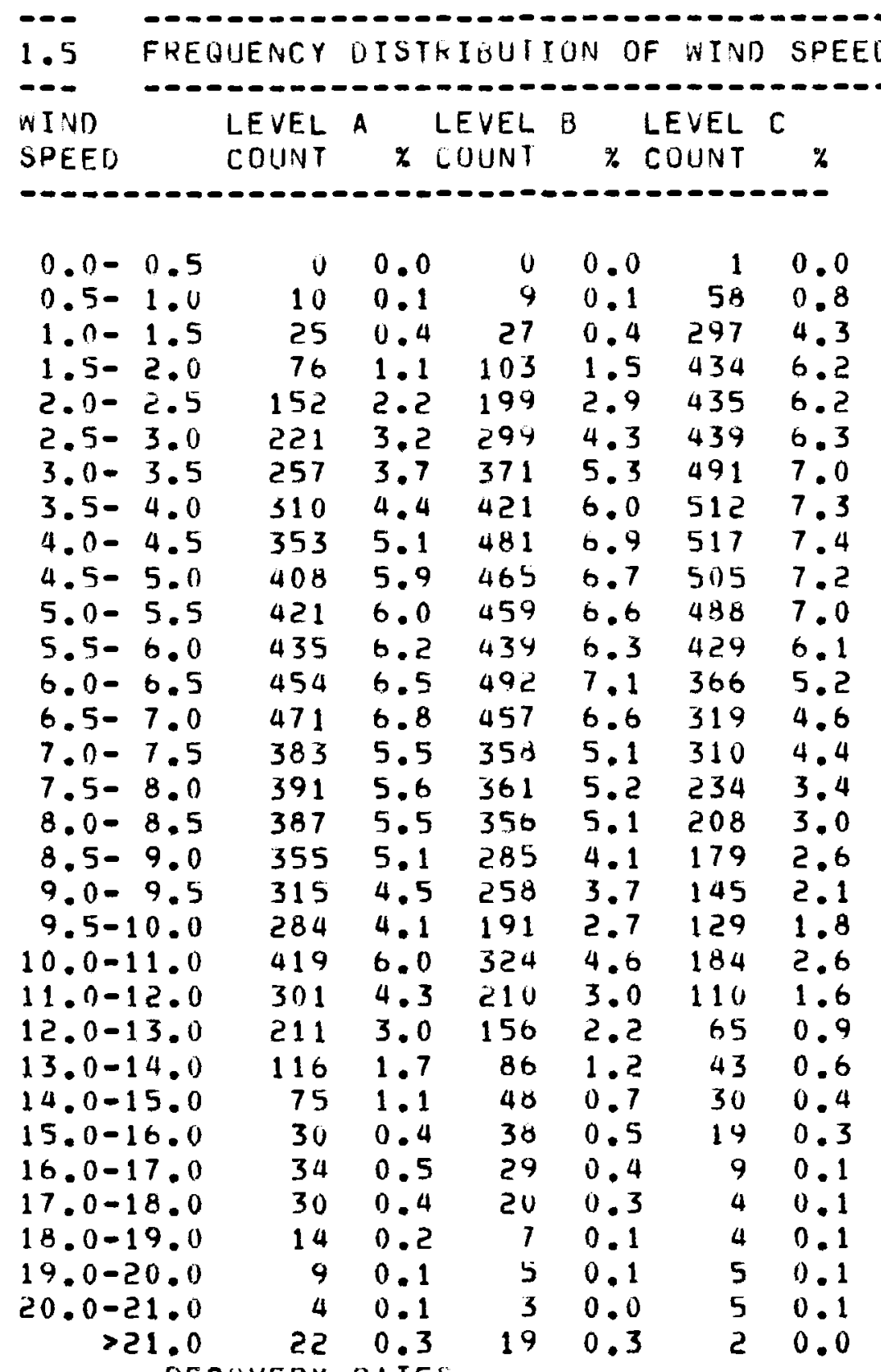

LEVEL A LEVEL B LEVEL C

$\begin{array}{lll}79.6 & 79.6 & 79.6\end{array}$ 
SITE ID:

INM

SITE LOCATION: CLAYTON, NM.

UATA:

JANUARY

1979 THROUGH DECEMBEF

1979

LEVEL A

\section{WIND}

SPEFD

-.-

0.5

1.0

1.5

2. 0

2.5

3.0

3.5

4.0

4.5

5.0

5.5

6. 0

6.5

7.0

7.5

8.0

8.5

9.0

9.5

10.0

11.0

12.0

13.0

14.0

15.0

16.0

17.0

18.0

19.0

20.0

21.0

$>21.0$

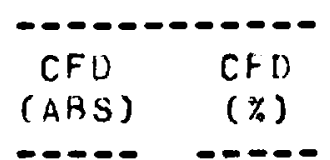

$0 \quad 0.00$

$10 \quad 0.14$

350.50

$111 \quad 1.59$

$263 \quad 3.77$

$484 \quad 6.94$

$741 \quad 10.63$

$1051 \quad 15.07$

140420.15

$1812 \quad 25.99$

$2233 \quad 32.02$

$2668 \quad 38.26$

$3122 \quad 44.71$

$3593 \quad 51.53$

$3976 \quad 57.02$

$4367 \quad 62.63$

$4754 \quad 68.16$

$5109 \quad 73.27$

$5424 \quad 77.79$

$5708 \quad 81.86$

$6127 \quad 87.87$

642892.18

$6639 \quad 95.21$

$6755 \quad 96.87$

$6830 \quad 97.95$

$6860 \quad 98.38$

689498.87

$6924 \quad 99.30$

$6938 \quad 99.50$

$6947 \quad 99.63$

095199.68

6973100.00

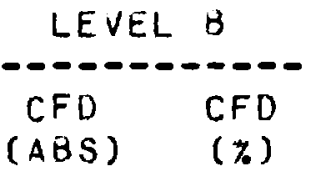

(ABS)
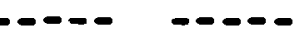

$$
9
$$

90.13

$36 \quad 0.52$

$139 \quad 1.99$

$338 \quad 4.85$

6379.13

$1008 \quad 14.45$

142920.48

$\begin{array}{ll}1910 & 27.38\end{array}$

$2375 \quad 34.05$

2B34

3273

3765

4222

4580

4941

5297

5582

5840

6031

6355

6565

6721

6807

6855

6893

6922

6942

6949

6954

6957

6976
40.62

46.92

53.97

60.52

65.65

70.83

75.93

80.02

83.72

66.45

91.10

94.11

96.34

97.58

98.27

98.81

99.23

99.51

99.61

99.68

99.73

100.00
LEVEL C

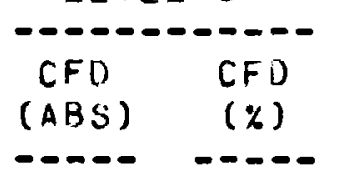

$$
10.01
$$

590.85

$356 \quad 5.10$

$790 \quad 11.32$

$1225 \quad 17.56$

166423.85

$2155 \quad 30.89$

266738.23

$3184 \quad 45.64$

368952.88

$4177 \quad 59.88$

$4606 \quad 66.03$

$4972 \quad 71.27$

529175.85

560180.29

$5835 \quad 83.64$

604386.63

$6222 \quad 89.19$

$\begin{array}{ll}6367 & 91.27\end{array}$

649693.12

$6680 \quad 95.76$

$6790 \quad 97.33$

685598.27

$6898 \quad 98.88$

$6928 \quad 99.31$

694799.58

$6956 \quad 99.71$

$6960 \quad 99.77$

690499.83

$6969 \quad 99.90$

$6974 \quad 99.97$

$6976 \quad 100.00$ 
SITE ID: $\quad$ NM

SITE LOCATION: CLAYTON, NM.

DATA : JANUARY 1979 THROUGH DECEMBER 1979

1.7 WIND SPEED PERSISTENCE FREQUENCY (LEVEL A)

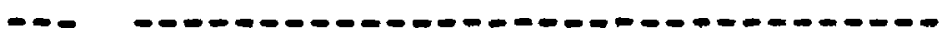

(LEVEL A)

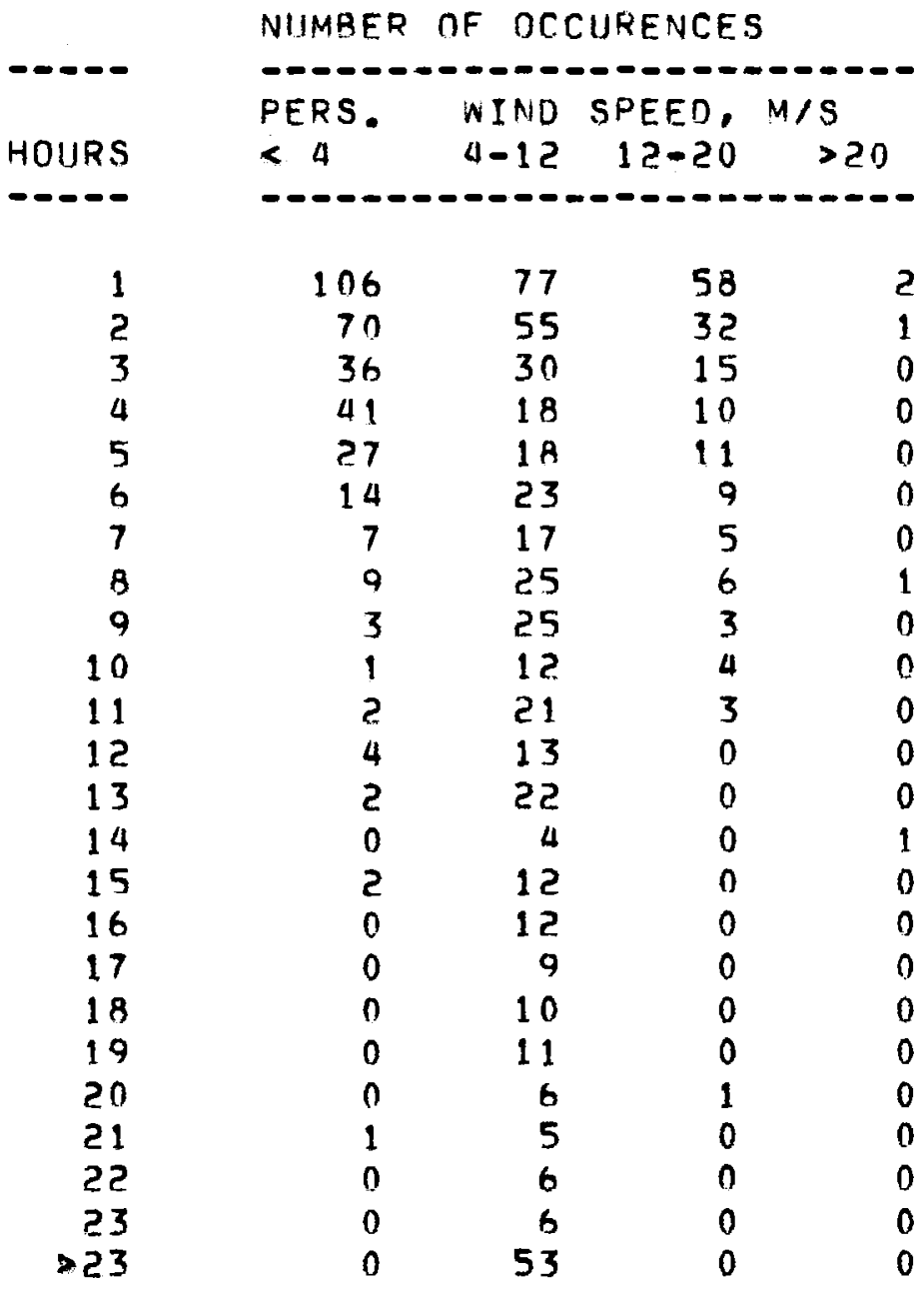




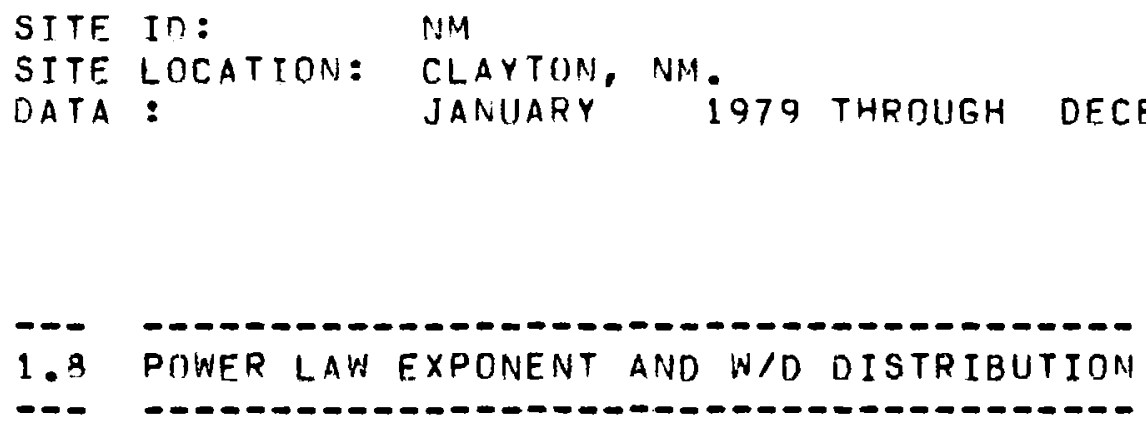

WIND DIR (ELEV A)

ALPHA ALPHA ALPHA ALPHA

$(A, B)(B, C)(A, C)(A, B, C) \quad \% A \quad \% B \quad \% C$

$\begin{array}{lrllllll}\text { N } & -0.16 & 0.17 & 0.17 & 0.17 & 6.71 & 6.59 & 6.36 \\ \text { NNE } & 0.15 & 0.15 & 0.15 & 0.15 & 3.80 & 4.69 & 4.13 \\ \text { NE } & 0.01 & 0.18 & 0.13 & 0.14 & 2.67 & 2.52 & 2.41 \\ \text { ENE } & 0.09 & 0.22 & 0.18 & 0.19 & 2.34 & 2.49 & 2.42 \\ \text { E } & 0.26 & 0.15 & 0.18 & 0.18 & 2.52 & 2.54 & 2.51 \\ \text { ESE } & 0.18 & 0.14 & 0.15 & 0.15 & 3.20 & 2.90 & 3.17 \\ \text { SE } & 0.29 & 0.11 & 0.16 & 0.15 & 4.43 & 3.81 & 4.57 \\ \text { SSE } & 0.12 & 0.17 & 0.16 & 0.16 & 8.05 & 7.60 & 8.11 \\ \text { S } & 0.20 & 0.15 & 0.16 & 0.16 & 14.01 & 13.69 & 13.46 \\ \text { SSW } & 0.05 & 0.20 & 0.16 & 0.17 & 10.63 & 11.22 & 10.44 \\ \text { SW } & 0.26 & 0.26 & 0.26 & 0.26 & 9.85 & 9.83 & 10.02 \\ \text { WSW } & 0.36 & 0.30 & 0.32 & 0.31 & 8.27 & 10.46 & 9.68 \\ \text { W } & 0.41 & 0.39 & 0.39 & 0.39 & 3.57 & 4.77 & 4.66 \\ \text { WNW } & 0.36 & 0.29 & 0.31 & 0.30 & 4.13 & 4.42 & 4.80 \\ \text { NW } & -0.06 & 0.19 & 0.13 & 0.14 & 6.15 & 6.57 & 7.01 \\ \text { NNW } & 0.31 & 0.12 & 0.17 & 0.16 & 6.27 & 5.89 & 6.25\end{array}$

\section{NOTES:}

$$
\text { 1. } \begin{aligned}
& W S(U P) \\
& W S(L O) \\
& W(U P)^{A L P H A}
\end{aligned}
$$$$
\text { 2. } \triangle L P H A=\frac{\operatorname{LOG}(W S(U P) / W S(L O))}{\operatorname{LOG}(Z(U P) / Z(L O))}
$$ 
COUNT OF VIND SPEED VS. DIRECTION

ERCH LINE DF CHARACTERS REPRESENTS 40 OQSERVATIONS

SPEED INCREASES WITH DISTANCE FROM CENTER OF CIRCLES

ASSOCIATED RANGES IN MPS ARE $0-4 \quad 4-10 \quad 10-20 \quad 20-$

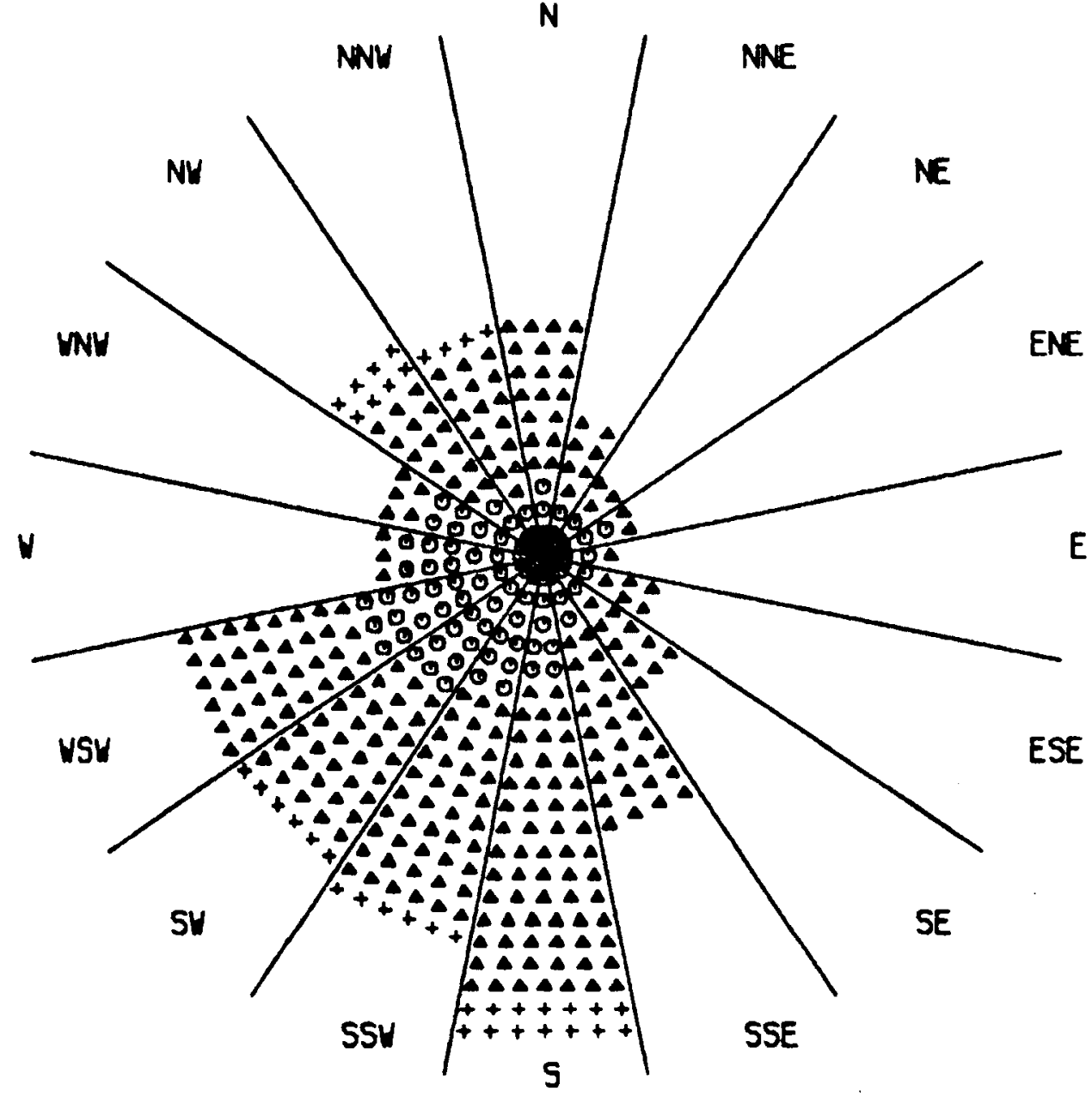

FIGURE 1. (NM) Wind Rose for Lowest Sensor Level 
COLD BAY, ALASKA 


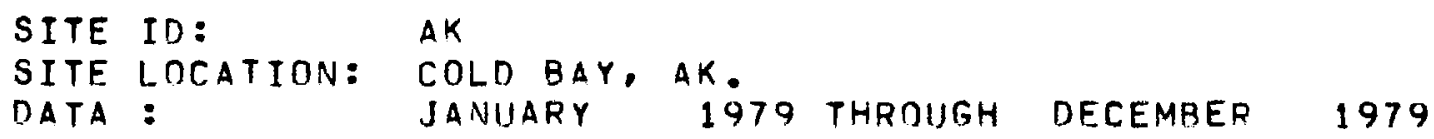

TOTAL POSSIBLE SAMPLES: 8760

$\begin{array}{lcc}\text { SENSOR } & \text { Y ON-LINE } & \text { X RECOVERED } \\ & & \\ \text { WS(A) } & 100.0 & 64.5 \\ \text { WD(A) } & 100.0 & 63.5 \\ \text { WS(C) } & 100.0 & 63.4 \\ \text { WD(C) } & 100.0 & 53.8\end{array}$

\begin{tabular}{|c|c|c|c|c|c|}
\hline (A) 21.8 & 22.9 & 137.4 & $11 / 09 / 79$ & $14: 00$ & (B) \\
\hline 9.1 & 16.8 & 147.3 & $11 / 09 / 79$ & $15: 00$ & $\begin{array}{l}\text { (A) } \\
\text { (B) }\end{array}$ \\
\hline
\end{tabular}

NOTES:

1. SITE ELEVATION: 29 METERS ABOVE SEA LEVEL.

3. SENSOR LEVEL B NOT AVAILABLE AT SITE AK. 
SITE IO: $\quad$ SK

SITE LOCATION: COLD BAY, AK.

JANLIARY

1979 THRDUGH DECEMBER

1979

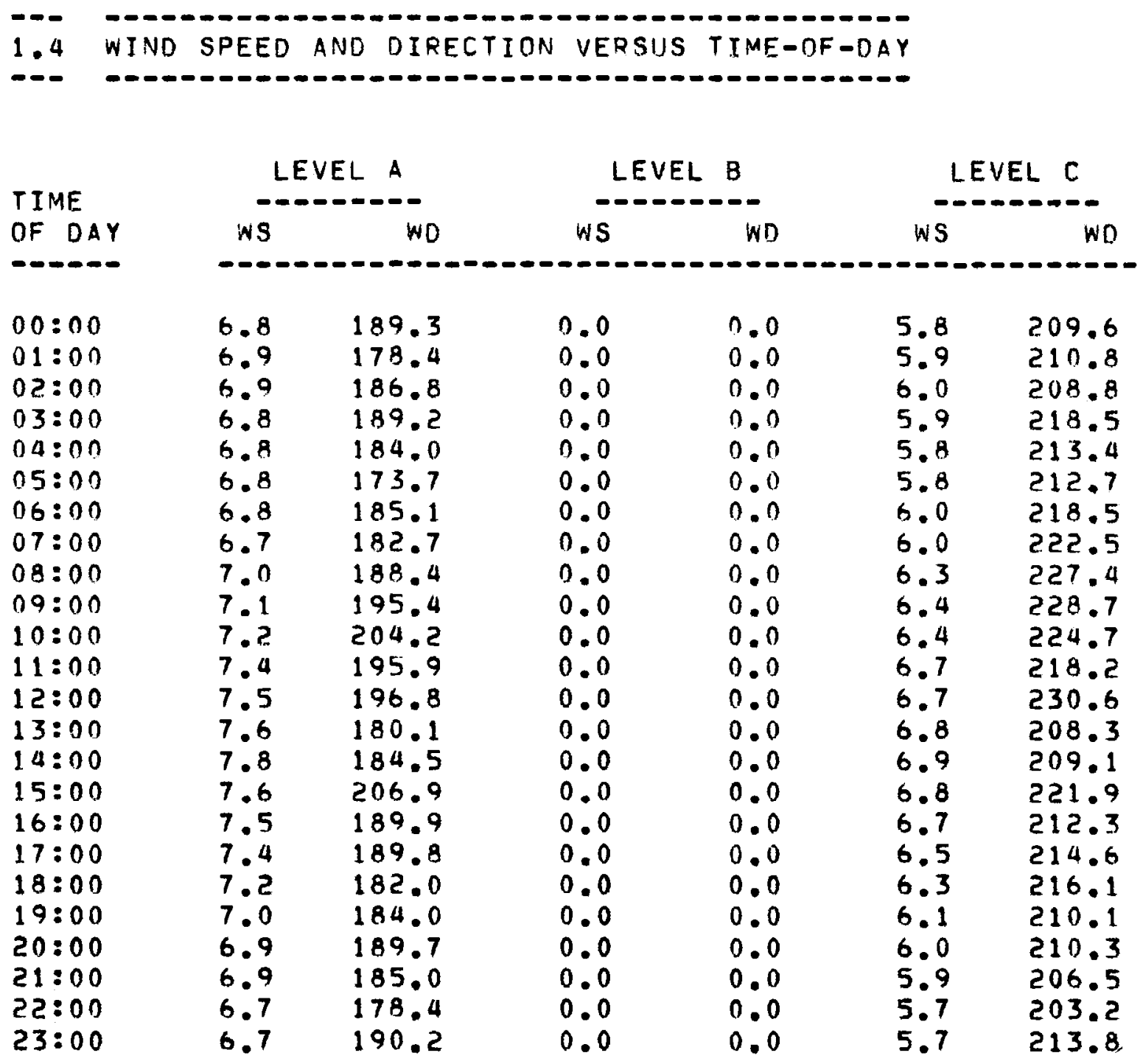


$\begin{array}{ll}\text { SITE ID: } & \text { AK } \\ \text { SITE LOCATION: } & \text { COLD BAY, AK. }\end{array}$

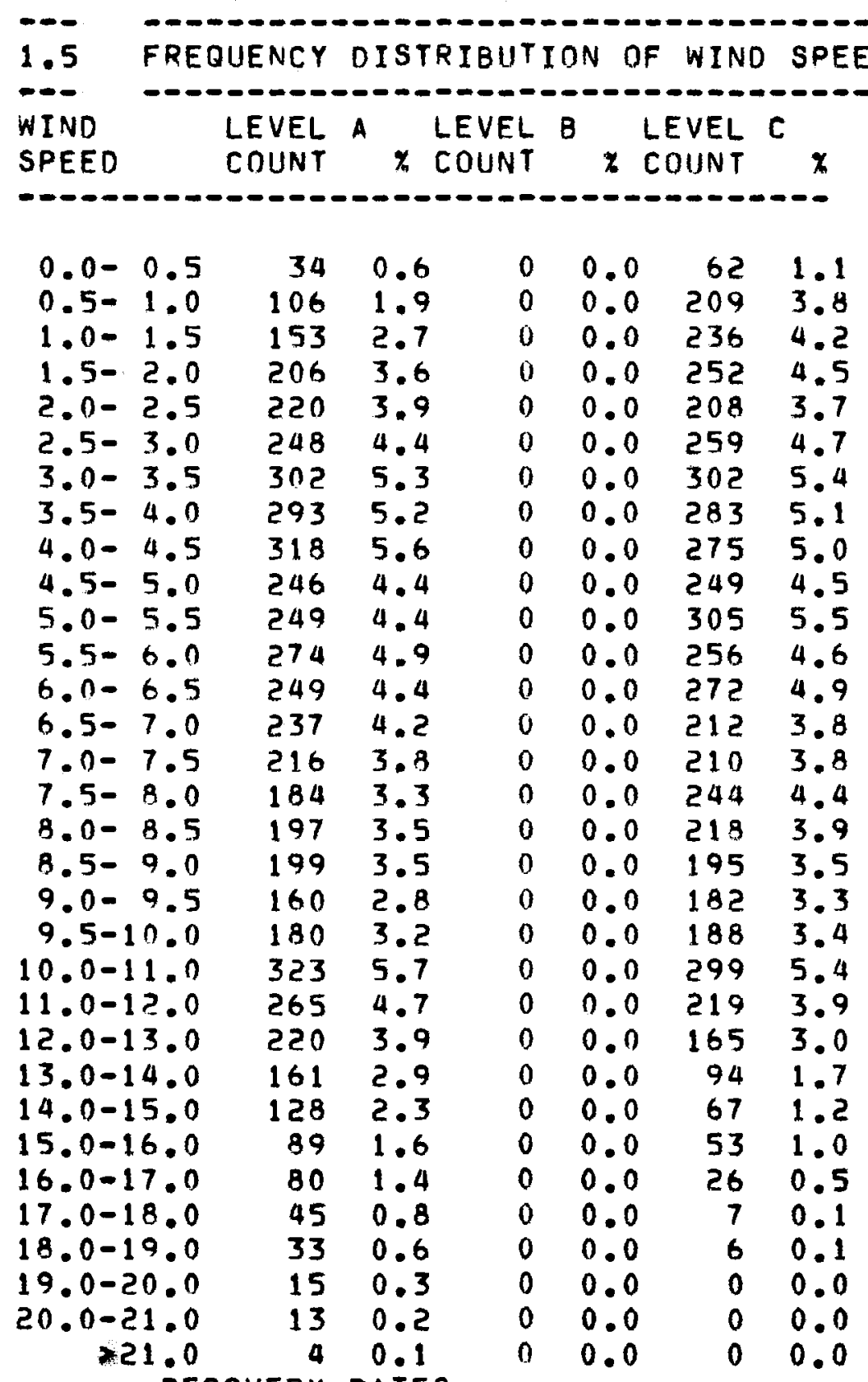

LEVEL A LEVEL B LEVEL C

$$
64.5 \quad 0.0 \quad 63.4
$$


$\begin{array}{lllll}\text { SITE ID: } & \text { AK } & & & \\ \text { SITE LOCATION: } & \text { COLD BAY, AK, } & & \\ \text { DATA : } & \text { JANUARY } 1979 & \text { THROUGH DECEMRER } & 1979\end{array}$

1.6 CUMIJLATIVE FREQUENCY DISTRIBUTION DF WIND SPEFD

LEVEL A

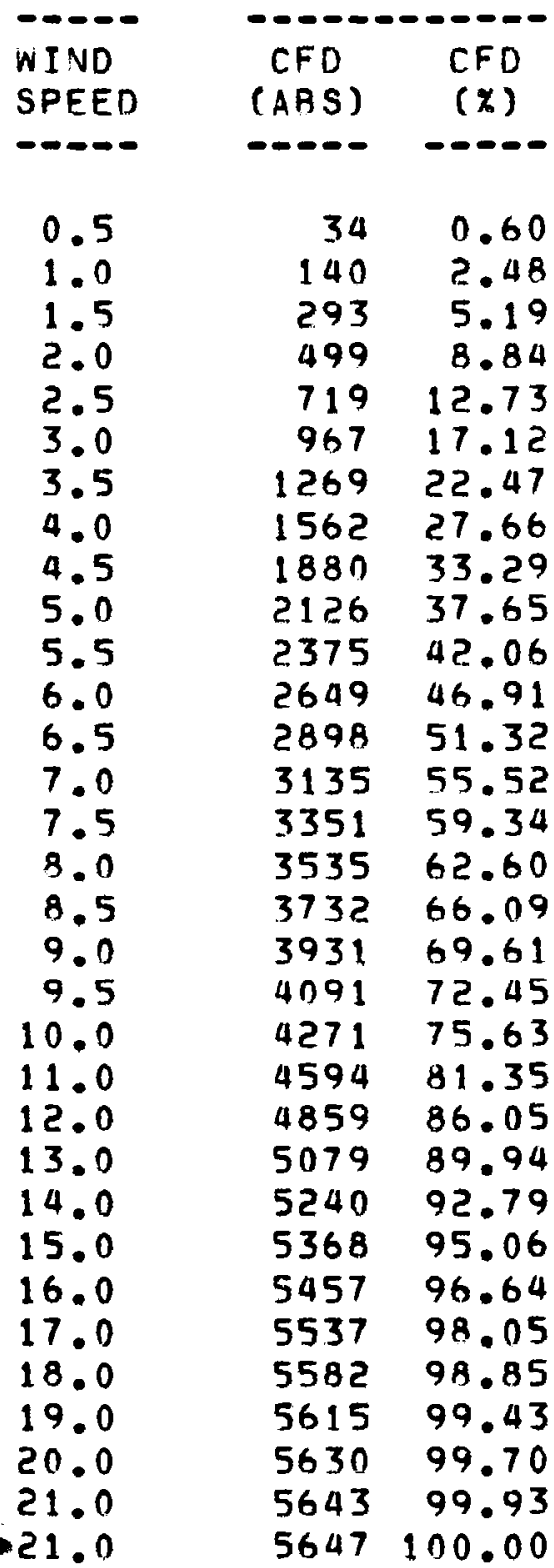

LEVEL 8

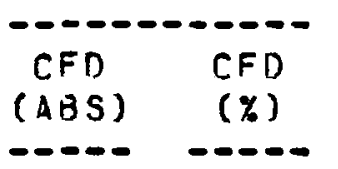

0
0.00

$0 \quad 0.00$

$0 \quad 0.00$

$0 \quad 0.00$

$0 \quad 0.00$

$0 \quad 0.00$

$0 \quad 0.00$

$0 \quad 0.00$

$0 \quad 0.00$

$0 \quad 0.00$

$0 \quad 0.00$

$0 \quad 0.00$

$0 \quad 0.00$

$0 \quad 0.00$

$0 \quad 0.00$

$0 \quad 0.00$

$0 \quad 0.00$

$0 \quad 0.00$

$0 \quad 0.00$

$0 \quad 0.00$

$0 \quad 0.00$

$0 \quad 0.00$

$0 \quad 0.00$

$0 \quad 0.00$

$0 \quad 0.00$

$0 \quad 0.00$

$0 \quad 0.00$

$0 \quad 0.00$

$0 \quad 0.00$

$0 \quad 0.00$

$0 \quad 0.00$

$0 \quad 0.00$
LEVEL C

\begin{tabular}{lc}
$C F D$ & $C F D$ \\
$(A B S)$ & $(\%)$ \\
\hdashline--- & ---- \\
62 & 1.12 \\
271 & 4.88 \\
507 & 9.13 \\
759 & 13.67 \\
967 & 17.41 \\
1226 & 22.08 \\
1528 & 27.52 \\
1811 & 32.61 \\
2086 & 37.57 \\
2335 & 42.05 \\
2640 & 47.54 \\
2896 & 52.15 \\
3168 & 57.05 \\
3380 & 60.87 \\
3590 & 64.65 \\
3834 & 69.04 \\
4052 & 72.97 \\
4247 & 76.48 \\
4429 & 79.76 \\
4617 & 83.14 \\
4916 & 88.53 \\
5135 & 92.47 \\
5300 & 95.44 \\
5394 & 97.14 \\
5461 & 98.34 \\
5514 & 99.30 \\
5540 & 99.77 \\
5547 & 99.89 \\
5553 & 100.00 \\
5553 & 100.00 \\
5553 & 100.00 \\
5553 & 100.00
\end{tabular}


SITE ID: AK

SITE LOCATION: COLO BAY, AK.

DATA: JANUARY

1979 THROUGH DECEMBER 1979

1.7

WIND SPEED PERSISTENCE FREQUENCY

(LEVEL A)

$---$

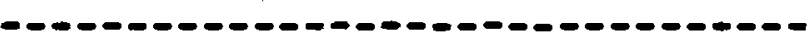

(LEVEL AJ

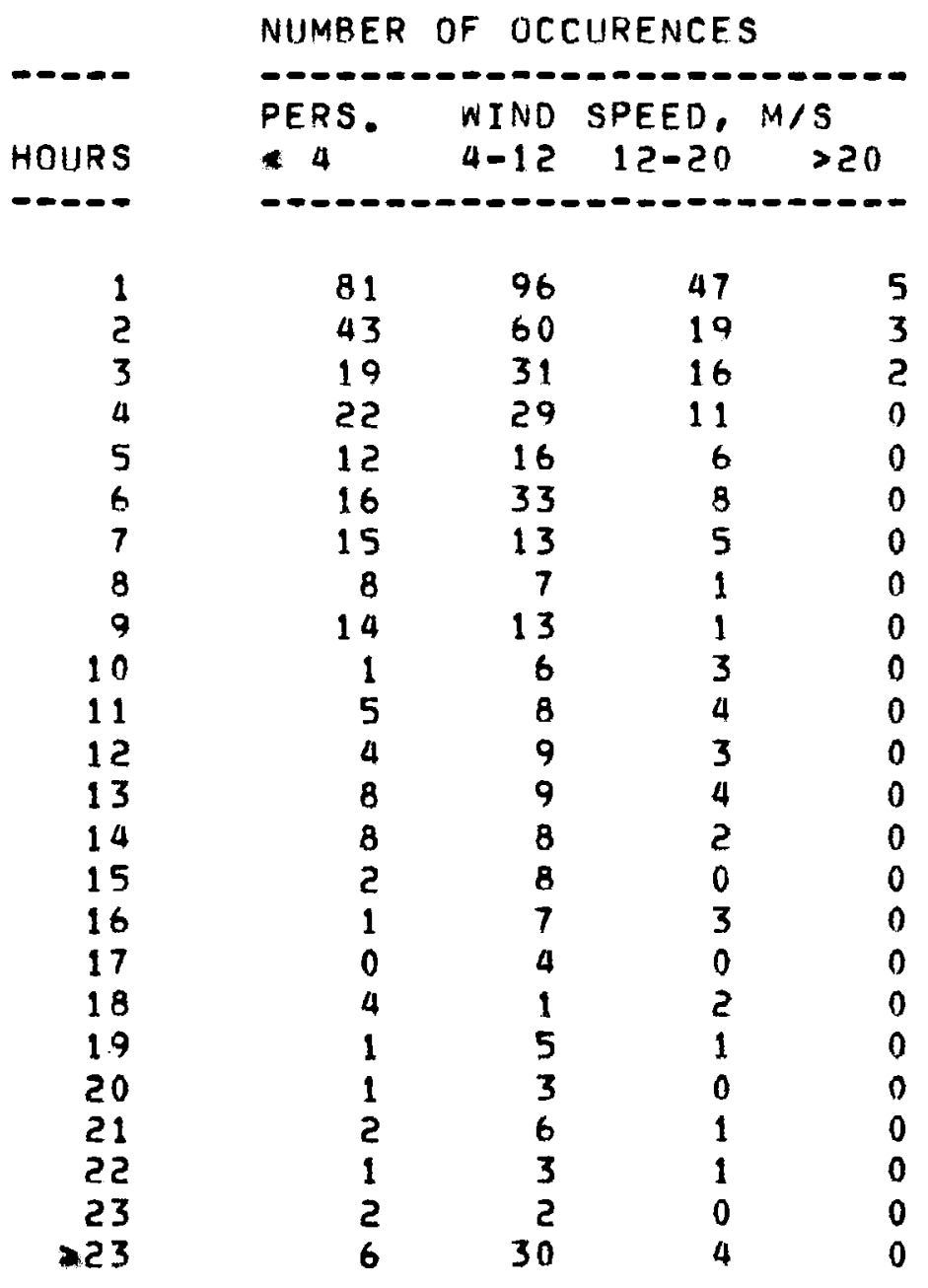


SITE ID: AK

SITE LOCATION: COLD BAY, AK.

JANUARY

1979 THROUGH DECEMBER

1979

1.8 POWER LAW EXPONENT AND W/D DISTRIBUTION

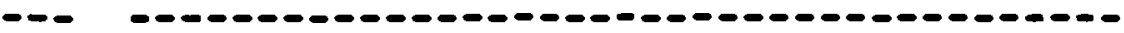

WIND DIR

(ELEV A)

ALPHA ALPHA ALPHA ALPHA

$(A, B)(B, C)(A, C)(A, B, C) \quad \% A \quad \% B \quad \% C$
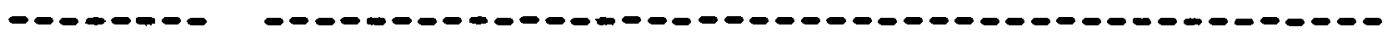

$\begin{array}{lrrrrrrr}\text { N } & 0.00 & 0.00 & 0.00 & 0.00 & 2.16 & 0.00 & 2.63 \\ \text { NNE } & 0.00 & 0.00 & -0.13 & 0.00 & 0.89 & 0.00 & 0.72 \\ \text { NE } & 0.00 & 0.00 & 0.02 & 0.00 & 1.54 & 0.00 & 1.02 \\ \text { ENE } & 0.00 & 0.00 & 0.05 & 0.00 & 2.83 & 0.00 & 2.11 \\ \text { E } & 0.00 & 0.00 & 0.00 & 0.00 & 3.35 & 0.00 & 2.38 \\ \text { ESE } & 0.00 & 0.00 & 0.22 & 0.00 & 13.60 & 0.00 & 8.00 \\ \text { SE } & 0.00 & 0.00 & 0.26 & 0.00 & 19.64 & 0.00 & 15.33 \\ \text { SSE } & 0.00 & 0.00 & -0.10 & 0.00 & 3.93 & 0.00 & 7.65 \\ \text { S } & 0.00 & 0.00 & 0.16 & 0.00 & 1.42 & 0.00 & 1.53 \\ \text { SSW } & 0.00 & 0.00 & -0.22 & 0.00 & 1.86 & 0.00 & 1.69 \\ \text { SW } & 0.00 & 0.00 & -0.57 & 0.00 & 5.31 & 0.00 & 3.91 \\ \text { WSW } & 0.00 & 0.00 & 0.05 & 0.00 & 7.54 & 0.00 & 5.89 \\ \text { W } & 0.00 & 0.00 & 0.13 & 0.00 & 10.01 & 0.00 & 7.94 \\ \text { WNW } & 0.00 & 0.00 & 0.16 & 0.00 & 9.24 & 0.00 & 9.29 \\ \text { NW } & 0.00 & 0.00 & 0.14 & 0.00 & 8.16 & 0.00 & 8.50 \\ \text { NNW } & 0.00 & 0.00 & 0.14 & 0.00 & 5.83 & 0.00 & 5.06\end{array}$

NOTES:

$$
\text { 1. } \frac{\text { WS (UP) }}{W S(L O)}=\frac{Z(U P)^{A L P H A}}{Z(L O)}
$$

2. ALPHA $=$ LOG (WS (UP)/WS $(L O))$

$\operatorname{LOG}(Z(U P) / Z(L O))$

WHERE; $\quad Z=E L E V A T I O N$

WS $=W I N D$ SPEED 
COUNT OF VIND SPEED VS. DIRECTION

ERCH LINE OF CHARACTERS REPRESENTS 40 OBSERVATIONS

SPEED INCREASES WITH DISTANCE FROM CENTER OF CIRCLES

ASSOCIATED RANGES IN MPS ARE $0 \begin{array}{lllllll}4 & -4 & -10 & 10 & -20 & 20-\end{array}$

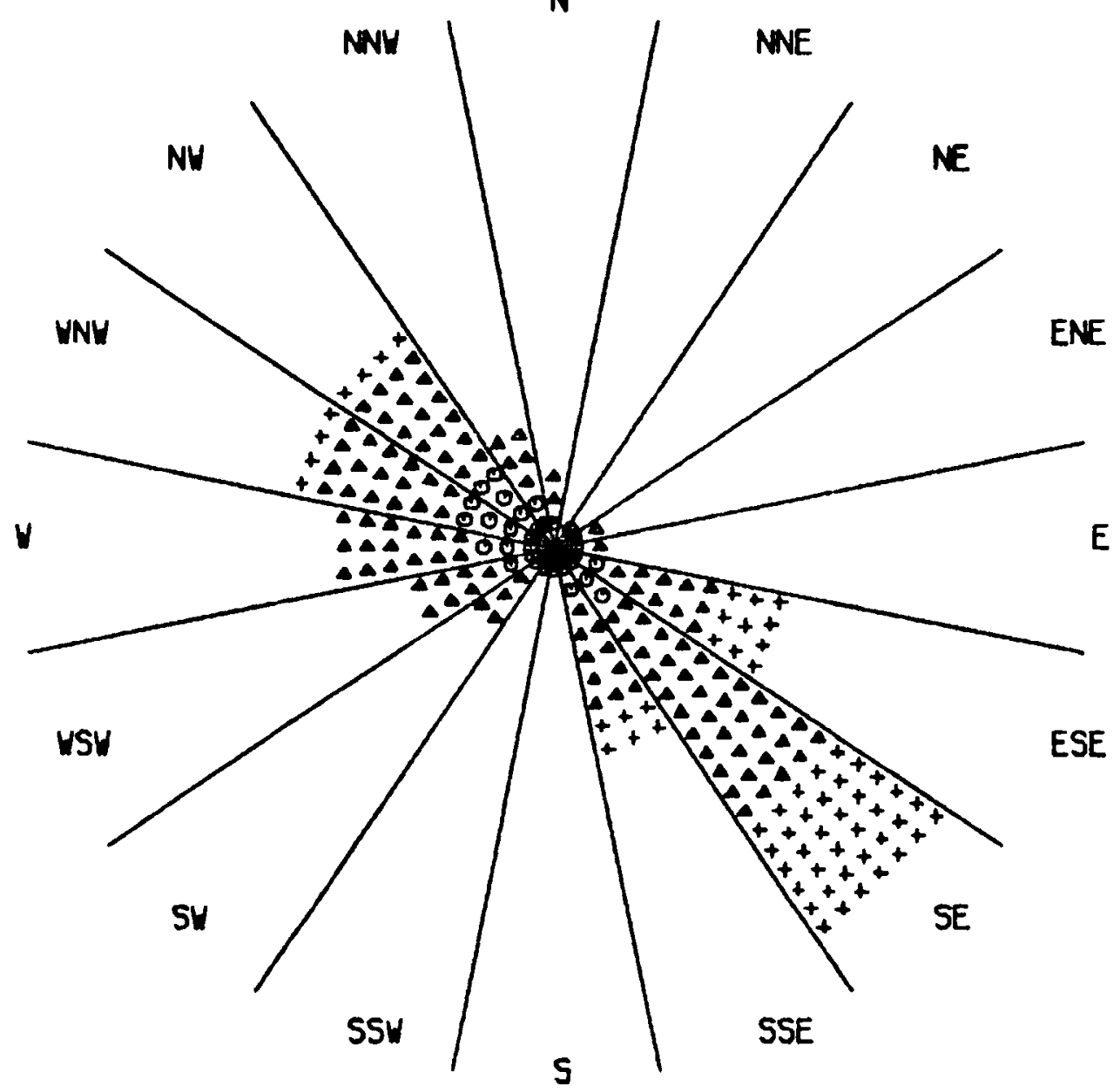

FIGURE 1. (AK) Wind Rose for Lowest Sensor Level 


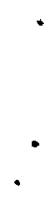


CULEBRA, PUERTO RICO 
$\begin{array}{lllll}\text { SITE IO: } & \text { PR } & & & \\ \text { SITE LOCATION: } & \text { CULEOKA, PR. } & & \\ \text { UATA : } & \text { JANIIARY } 1979 & \text { THROUGH DECEMGER } & 1979\end{array}$

1.1 SENSOR PFRFORMANCE

TOTAL POSSIELE SAMPLES: 8760

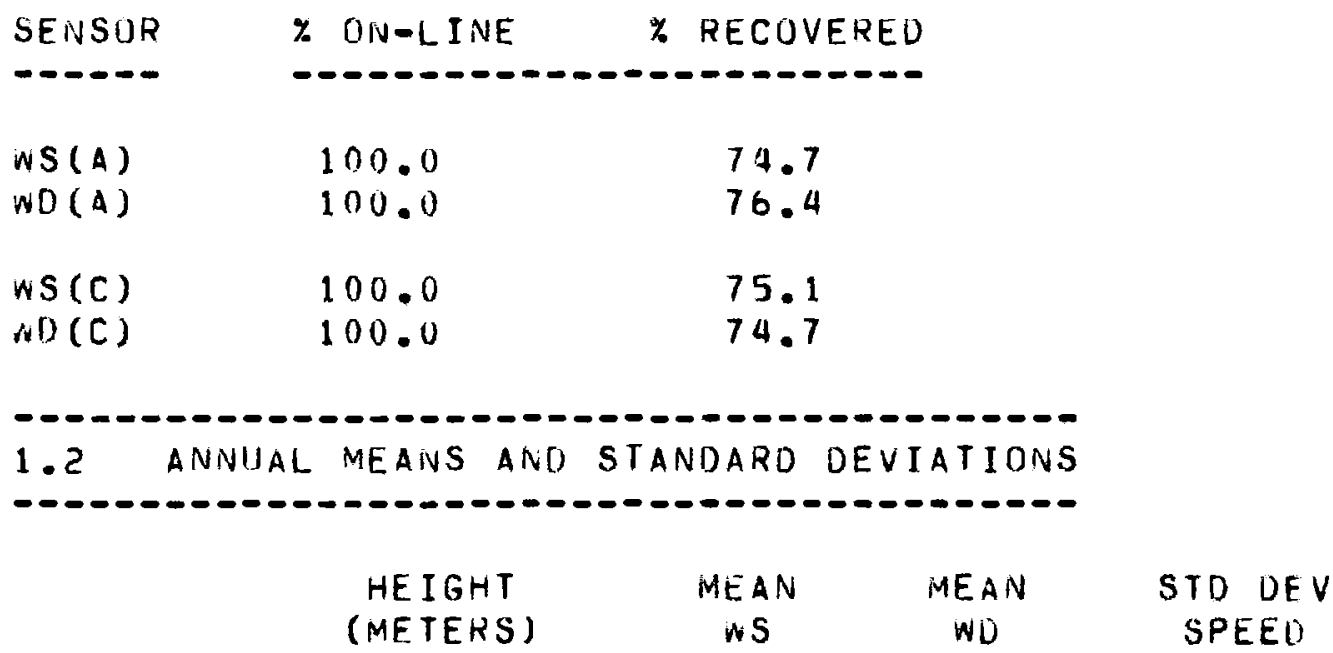

SITE DATA

(A) 45.7

7.4

79.5

81.7

2.6

6.5

2.5

1.3 MAXIMUM NIND SHEEO

$-0-0-0-0-0-0-0-0-0-0-0$

ELEVATION

(METERS)
(4) 45.7
31.7
05.
$09 / 01 / 79$
$02: 00$
(B) -999.9
(C) 9.1
29.1
70.2
$09 / 01 / 79$
$02: 00$
(C) 29.1
(A) 31.7
(B) -999.9

$-0-0-0-0-0-0-0-0-0-0-0-0-0-0-0-0$

NOTES:

1. Site elevation: 80 meters above Sea leVel.

3. SENSUR LEVEL B NOT AVAILABLE AT SITE PR. 
$\begin{array}{lllll}\text { SITE ID: } & \text { PR } & & & \\ \text { SITE LUCATIUN: } & \text { CULEGRA, PR, } & & \\ \text { OATA : } & \text { JANUARY } & \text { i979 THKOUGH DECEMBER } & 1979\end{array}$

1.4 WINU SPEEU ANU UIRFCTION VEKSUS TIME-OF-DAY

\begin{tabular}{|c|c|c|c|c|c|c|}
\hline & & $-A$ & & $B$ & & $-c$ \\
\hline TIME & - & $--\infty$ & & $-\infty$ & & $--\infty$ \\
\hline UF DAY & iv $S$ & WU & wS & WD & wS & WD \\
\hline$-\infty-\infty$ & $--\infty$ & & & & & -- \\
\hline $00: 00$ & 7.4 & 80.9 & 0.0 & 0.0 & 6.3 & 82.9 \\
\hline $01: 00$ & 7.3 & 80.4 & 0.0 & 0.0 & 6.2 & 83.0 \\
\hline $02: 00$ & 7.2 & 81.5 & 0.0 & 0.0 & 6.1 & 83.5 \\
\hline $03: 00$ & 7.2 & 80.4 & 0.0 & 0.0 & 6.1 & 82.9 \\
\hline $04: 00$ & 7.2 & 80.7 & 0.0 & 0.0 & 6.2 & 83.0 \\
\hline $05: 00$ & 7.3 & 81.4 & 0.0 & 0.0 & 6.2 & 83.4 \\
\hline $06: 00$ & 7.2 & 81.5 & 0.0 & 0.0 & 6.1 & 83.0 \\
\hline $07: 00$ & 7.1 & 80.2 & 0.0 & 0.0 & 6.3 & 80.2 \\
\hline $08: 00$ & 7.3 & 79.2 & 0.0 & 0.0 & 6.6 & 78.7 \\
\hline $09: 00$ & 7.6 & 78.2 & 0.0 & 0.0 & 6.9 & 79.2 \\
\hline $10: 00$ & 7.7 & 78.4 & 0.0 & 0.0 & 7.0 & 79.8 \\
\hline $11: 00$ & 7.8 & 70.4 & 0.0 & 0.0 & 7.0 & 80.6 \\
\hline $12: 00$ & 7.8 & 78.6 & 0.0 & 0.0 & 7.0 & 80.6 \\
\hline $13: 00$ & 7.6 & 79.1 & 0.0 & 0.0 & 6.9 & 80.6 \\
\hline $14: 00$ & 7.5 & 79.1 & 0.0 & 0.0 & 6.7 & 80.8 \\
\hline $15: 00$ & 7.4 & 79.9 & 0.0 & 0.0 & 6.5 & 81.0 \\
\hline $16: 00$ & 7.3 & 80.3 & 0.0 & 0.0 & 6.4 & 81.6 \\
\hline $17: 00$ & 7.4 & 80.5 & 0.0 & 0.0 & 6.4 & 82.5 \\
\hline $18: 00$ & 7.5 & 79.2 & 0.0 & 0.0 & 6.4 & 82.8 \\
\hline $19: 00$ & 7.6 & 78.0 & 0.0 & 0.0 & 6.5 & 82.6 \\
\hline $20: 00$ & 7.5 & 77.5 & 0.0 & 0.0 & 6.5 & 82.0 \\
\hline $21: 00$ & 7.5 & 77.6 & 0.0 & 0.0 & 6.5 & 81.9 \\
\hline $22: 00$ & 7.5 & 77.8 & 0.0 & 0.0 & 6.5 & 81.2 \\
\hline $23: 00$ & 7.4 & 79.8 & 0.0 & 0.0 & 6.3 & 82.6 \\
\hline
\end{tabular}


SITE ID: $\quad$ PR

SITE LOCATION: CULEURA, PR.

DATA : JANUARY 1979 THROUGH DECEMBEF 1979

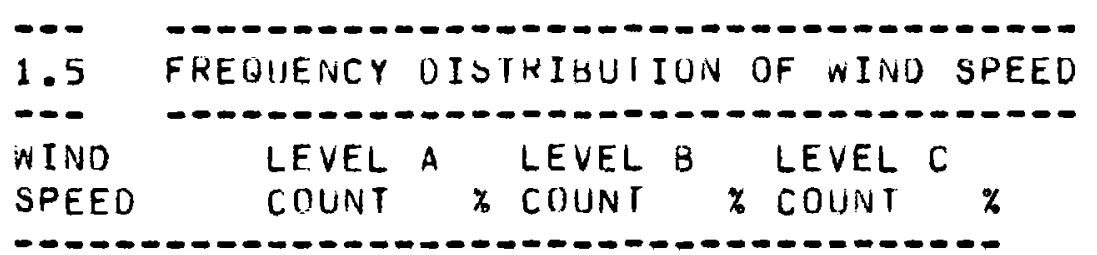

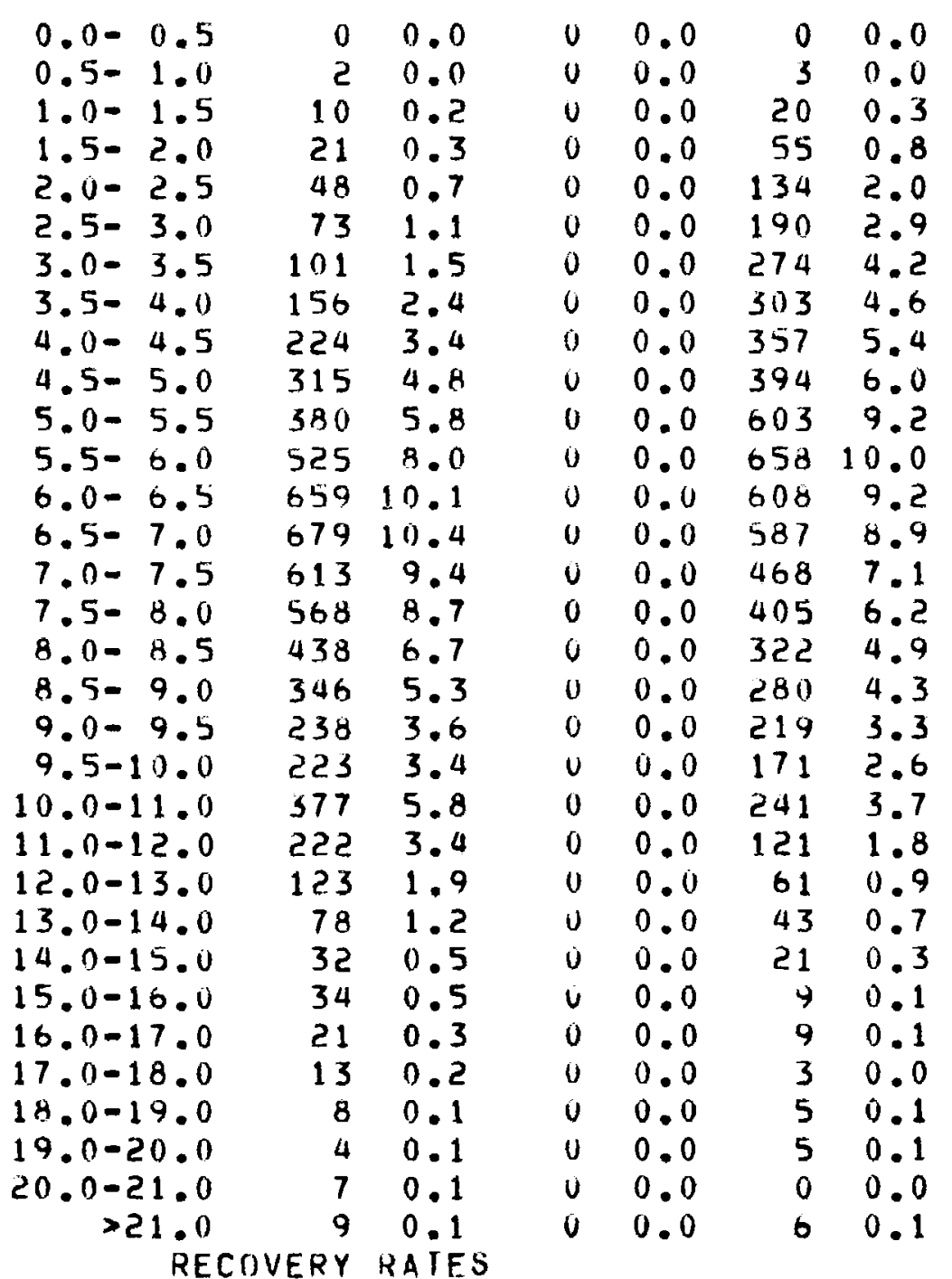

LEVEL A LEVEL B LEVEL C

$\begin{array}{ll}74.7 & 0.0\end{array}$ 
$\begin{array}{lllll}\text { SITE IO: } & \text { PR } & & & \\ \text { SITE LOCATIUN: } & \text { CULEORA, PR, } & & \\ \text { DATA : } & \text { JANUAKY } & \text { i979 THRDUGH DECEMEER } & 1979\end{array}$

1.b CUMULATIVE FREQUERTY DISTRIBUTION OF WIND SPEED

\begin{tabular}{|c|c|c|c|c|c|c|}
\hline----- & $\begin{array}{r}\text { LEV } \\
-\cdots-\end{array}$ & $\begin{array}{l}\text { L A } \\
-----\end{array}$ & $\begin{array}{l}\text { LEV } \\
\ldots-0 .\end{array}$ & $\begin{array}{l}B \\
---.\end{array}$ & $\begin{array}{r}\text { LEV } \\
----\end{array}$ & $\begin{array}{l}\mathrm{L} \\
-\end{array}$ \\
\hline $\begin{array}{l}\text { WI VD } \\
\text { SPEED }\end{array}$ & $\begin{array}{c}C F D \\
(A B S)\end{array}$ & $\begin{array}{l}\text { CFD } \\
(\%)\end{array}$ & $\begin{array}{l}C F O \\
(A B S)\end{array}$ & $\begin{array}{l}\text { CFD } \\
(\%)\end{array}$ & $\begin{array}{c}C F O \\
(A B S)\end{array}$ & $\begin{array}{l}C F D \\
(\%)\end{array}$ \\
\hline----- & ----- & $-\infty-\infty$ & $---\infty$ & $---\infty$ & $----\infty$ & -... \\
\hline 0.5 & 0 & 0.00 & 0 & 0.00 & 0 & 0.00 \\
\hline 1.0 & 2 & 0.03 & 0 & 0.00 & 3 & 0.05 \\
\hline 1.5 & 12 & 0.18 & 0 & 0.00 & 2.3 & 0.35 \\
\hline 2.0 & 33 & 0.50 & 0 & 0.00 & 78 & 1.19 \\
\hline 2.5 & 81 & 1.24 & 0 & 0.00 & 212 & 3.22 \\
\hline 3.0 & 154 & 2.35 & 0 & 0.00 & 402 & 6.11 \\
\hline 3.5 & 255 & 3.89 & 0 & 0.00 & 676 & 10.28 \\
\hline 4.0 & 411 & 6.23 & 0 & 0.00 & 979 & 14.89 \\
\hline 4.5 & 635 & 9.70 & 0 & 0.00 & 1336 & 20.32 \\
\hline 5.0 & 950 & 14.51 & 0 & 0.00 & 1730 & 26.31 \\
\hline 5.5 & 1330 & 20.31 & 0 & 0.00 & 2333 & 35.48 \\
\hline 6.0 & 1855 & 28.35 & 0 & 0.00 & 2991 & 45.49 \\
\hline 6.5 & 2514 & 38.40 & 0 & 0.00 & 3599 & 54.74 \\
\hline 7.0 & 3193 & 48.77 & 0 & $0.0 !$ & 4186 & 63.67 \\
\hline 7.5 & 3806 & 58.13 & 0 & 0.00 & 4654 & 70.78 \\
\hline 8.0 & 4374 & 66.81 & 0 & 0.00 & 5059 & 76.94 \\
\hline 8.5 & $481 ?$ & 73.50 & 0 & 0.00 & 5381 & 81.84 \\
\hline 9.0 & 5158 & 78.78 & 0 & 0.00 & 5661 & 86.10 \\
\hline 9.5 & 5396 & 82.42 & 0 & 0.00 & 5860 & 89.43 \\
\hline 10.0 & 5619 & 85.83 & 0) & 0.00 & 6051 & 92.03 \\
\hline 11.0 & 5996 & 91.58 & 0 & $0.0 u$ & 6242 & 95.70 \\
\hline 12.0 & 6218 & 911.97 & 0 & 0.00 & 6413 & 97.54 \\
\hline 13.0 & 6341 & 96.85 & 0 & 0.00 & 6474 & 98.46 \\
\hline 14.0 & 6419 & 98.04 & 0 & 0.00 & 6517 & 99.12 \\
\hline 15.0 & 6451 & 98.53 & 0 & 0.00 & 6538 & 99.44 \\
\hline 16.0 & 6485 & 99.05 & 0 & 0.00 & 6547 & 99.57 \\
\hline 17.0 & 0506 & 99.37 & 0 & 0.00 & 6556 & 99.71 \\
\hline 18.0 & 6519 & 99.57 & 0 & 0.00 & 6559 & 99.76 \\
\hline 19.0 & 6527 & 99.69 & 0 & 0.00 & 6564 & 99.83 \\
\hline 20.0 & 6531 & 99.76 & 0 & 0.00 & 6569 & 99.91 \\
\hline 21.0 & 6538 & 99.86 & 0 & 0.00 & 6569 & 99.91 \\
\hline 21.0 & 6547 & 100.00 & 0 & 0.00 & 6575 & 100.00 \\
\hline
\end{tabular}


SITE ID:

SITE LOCATION: CULEBRA, PR.
DATA :
$P R$
1979 THROUGH
DECEMAER

1979

1.7

WIND SPEEO PERSISTENCE FREQUENCY

(LEVEL A)

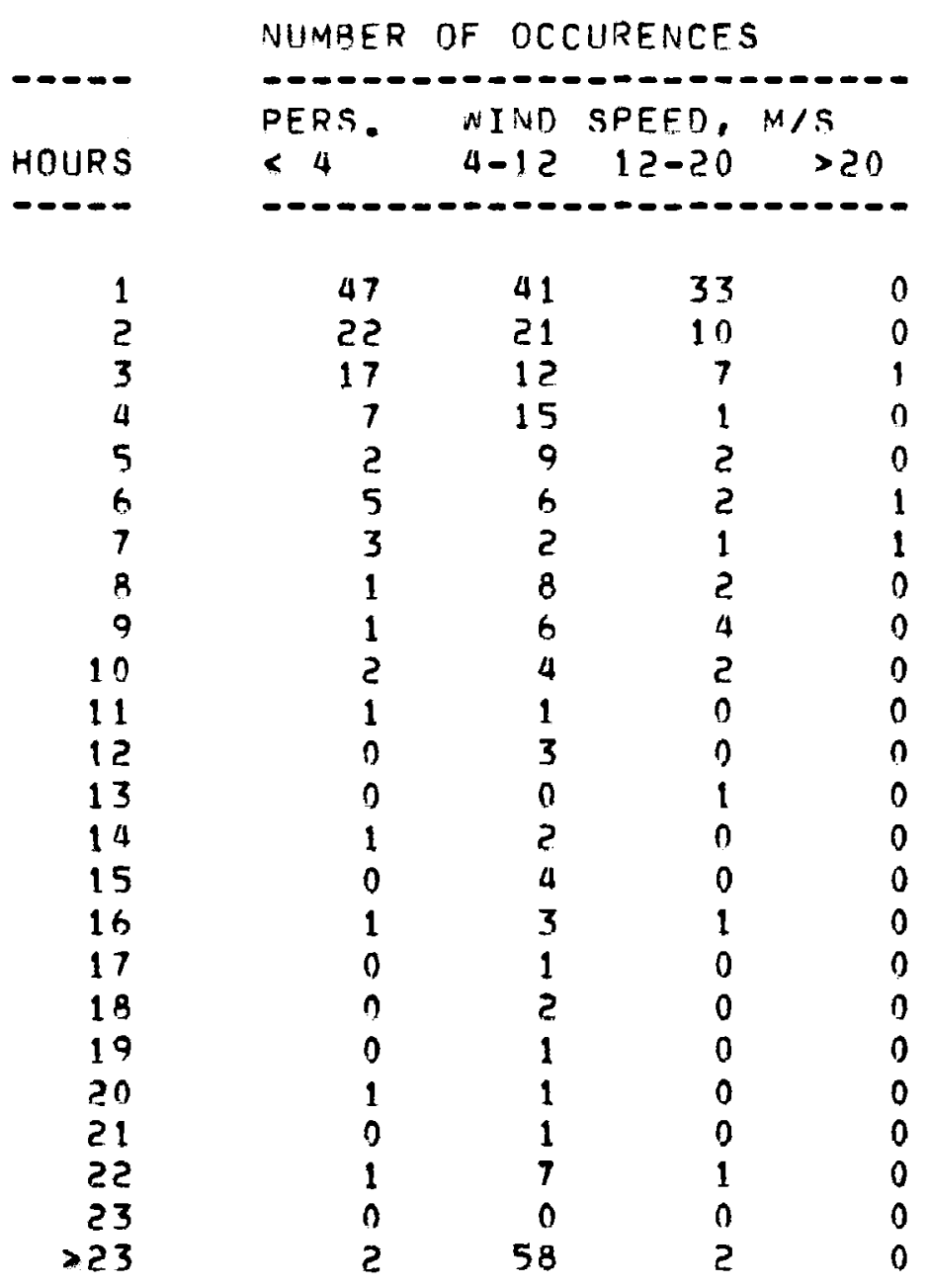

$P R=05$ 


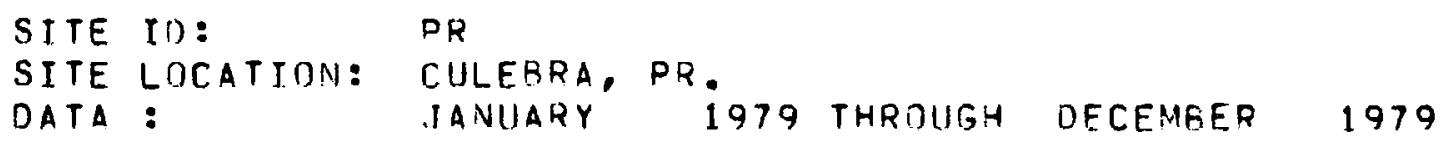

$\begin{array}{llllllll}\text { N } & 0.00 & 0.00 & 0.20 & 0.00 & 1.07 & 0.00 & 0.81 \\ \text { NNE } & 0.00 & 0.00 & 0.22 & 0.00 & 2.86 & 0.00 & 1.43 \\ \text { NE } & 0.00 & 0.00 & 0.15 & 0.00 & 12.07 & 0.00 & 7.32 \\ \text { ENE } & 0.00 & 0.00 & 0.03 & 0.00 & 36.67 & 0.00 & 35.65 \\ \text { E } & 0.00 & 0.00 & 0.06 & 0.00 & 27.48 & 0.00 & 38.16 \\ \text { ESE } & 0.00 & 0.00 & 0.17 & 0.00 & 11.01 & 0.00 & 7.42 \\ \text { SE } & 0.00 & 0.00 & 0.26 & 0.00 & 4.28 & 0.00 & 2.52 \\ \text { SSE } & 0.00 & 0.00 & 0.32 & 0.00 & 1.88 & 0.00 & 1.38 \\ \text { S } & 0.00 & 0.00 & 0.31 & 0.00 & 1.10 & 0.00 & 0.99 \\ \text { SSN } & 0.00 & 0.00 & 0.36 & 0.00 & 0.58 & 0.00 & 0.84 \\ \text { SW } & 0.00 & 0.00 & 0.01 & 0.00 & 0.14 & 0.00 & 0.44 \\ \text { WSW } & 0.00 & 0.00 & 0.21 & 0.00 & 0.06 & 0.00 & 0.17 \\ \text { W } & 0.00 & 0.00 & 0.00 & 0.00 & 0.09 & 0.00 & 0.08 \\ \text { WNW } & 0.00 & 0.00 & 0.22 & 0.00 & 0.08 & 0.00 & 0.09 \\ \text { WW } & 0.00 & 0.00 & 0.12 & 0.00 & 0.15 & 0.00 & 0.09 \\ \text { NNW } & 0.00 & 0.00 & 0.20 & 0.00 & 0.49 & 0.00 & 0.43\end{array}$

NOTES:

$$
\text { 1. } \frac{\text { WS (IJP) }}{\mathrm{NS}(L 0)}=\frac{Z(U P P)}{Z(L O)}
$$

\section{ALPHA}

$\operatorname{LOG}(W S(U P) / W S(L O))$

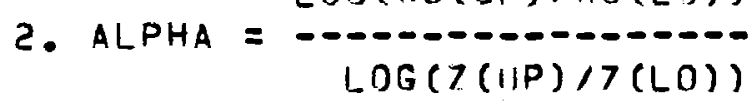

WHERE: $\quad Z=E L E V A T I D N$ WSEWIND SPEED 


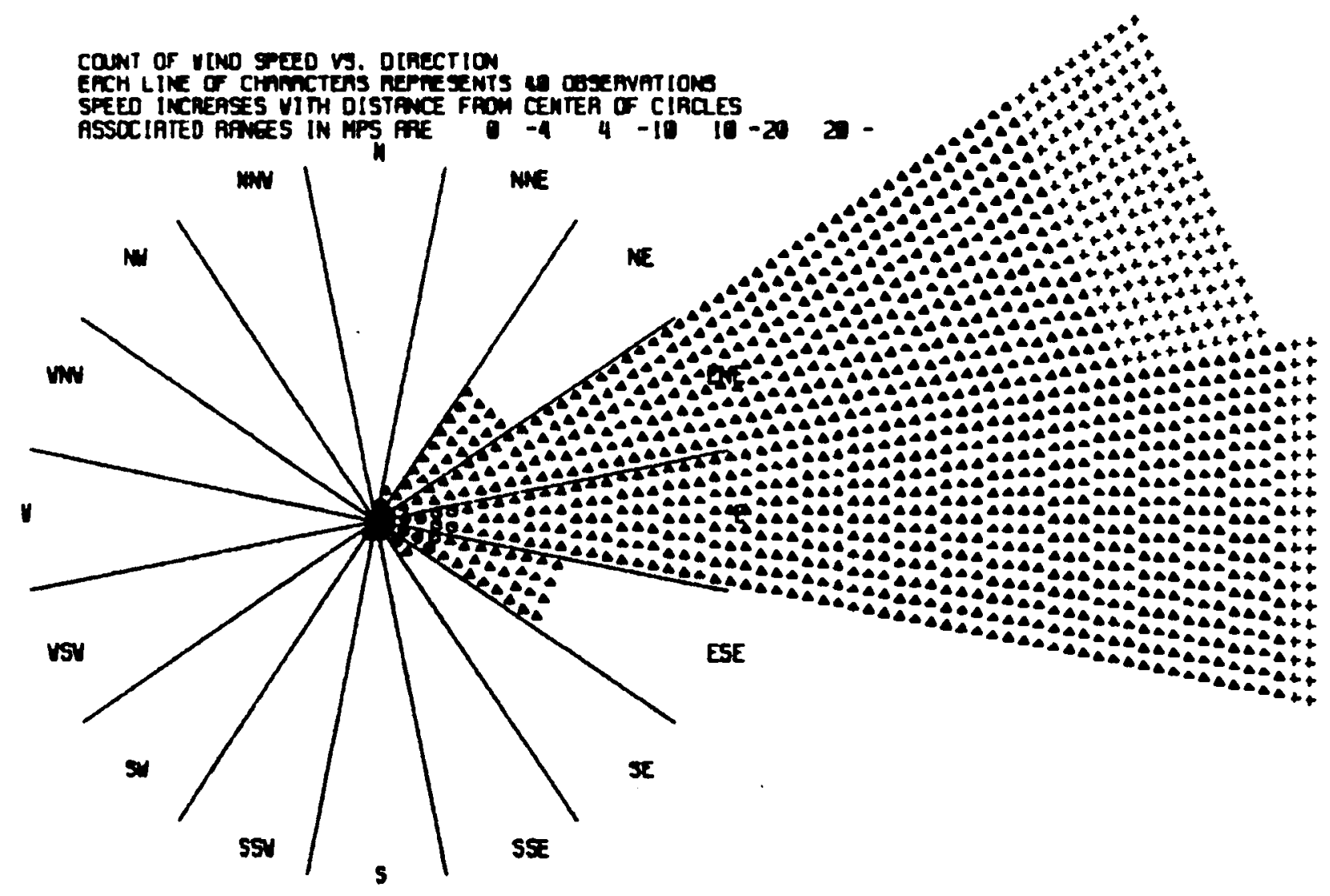

FIGURE 1. (PR) Wind Rose for Lowest Sensor Level 



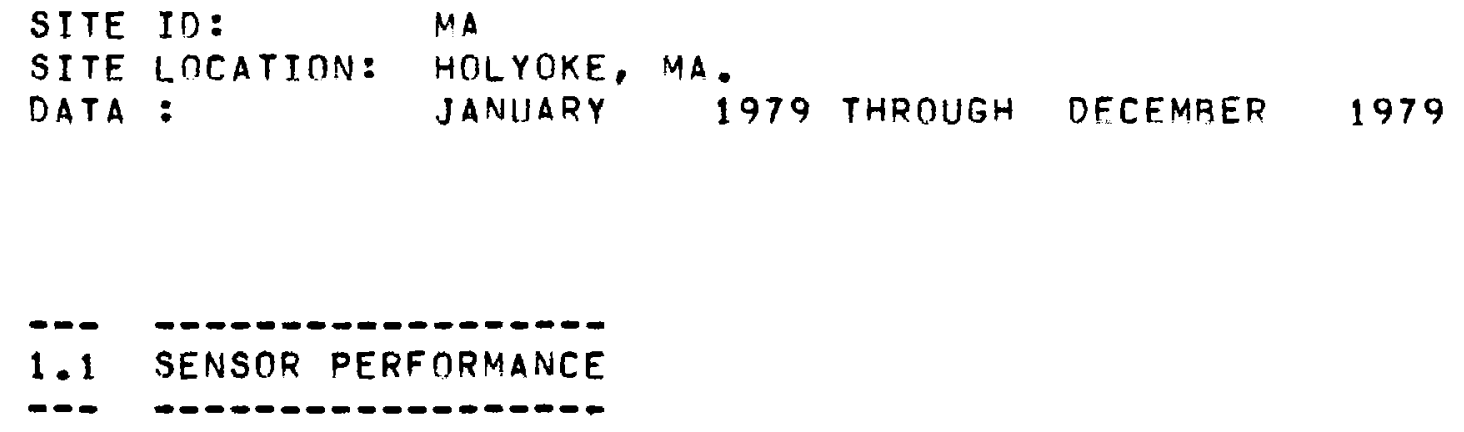

TOTAL POSSIBLE SAMPLES: $\quad 8760$

$\begin{array}{lcc}\text { SENSOR } & \times \text { ON-LINE } & \text { \% RECOVERED } \\ \text { WS(A) } & 100.0 & 93.5 \\ \text { WD(A) } & 100.0 & 93.5 \\ & & \\ \text { WS(C) } & 100.0 & 93.2 \\ \text { WD(C) } & 100.0 & 90.5\end{array}$

1.5

1.2 ANNIIAL MEANS AND STANDARD DEVIATIONS

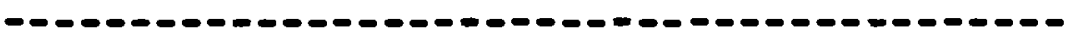

$\begin{array}{cccc}\text { HEIGHT } & \text { MEAN } & \text { MEAN } & \text { STD DEV } \\ \text { (METERS) } & \text { WS } & \text { WD } & \text { SPEED }\end{array}$

SITE DATA

(A) 45.7

$\begin{array}{ll}6.5 & 243.9 \\ 4.3 & 234.0\end{array}$

3.6

(C) 18.2

2.3

1.3 MAXIMUM WIND SPEED

1.

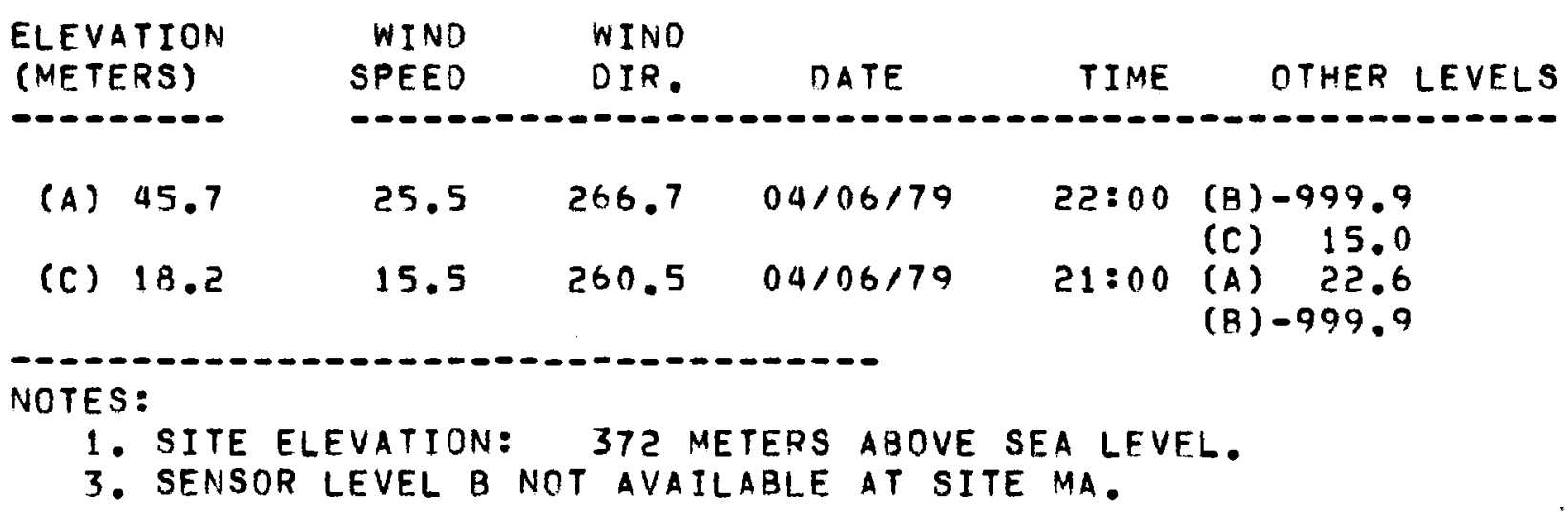


SITE ID:

SITE LOCATION: HOLYOKE, MA.

DATA : JANUARY 1979 THROUGH DECEMRER 1979

1.4 WIND SPEED AND DIRECTION VERSUS TIME-OF-DAY

$-0,--0-0-0---0-0-0-0-0-0-0-0-0-0-0-0-0-0-0-0-$

\begin{tabular}{|c|c|c|c|c|c|c|}
\hline $\begin{array}{l}\text { TIME } \\
\text { OF DAY }\end{array}$ & ws & $\begin{array}{l}E L A \\
\text { WD }\end{array}$ & ws & $\frac{B}{W D}$ & ws & L C $C$ \\
\hline------ & $-\infty$ & - - & & $-\infty$ & & \\
\hline $00: 00$ & 6.6 & $241 \cdot 2$ & 0.0 & 0.0 & 4.5 & 235.0 \\
\hline $02=00$ & $\begin{array}{l}6.5 \\
6.5\end{array}$ & $\begin{array}{l}247.2 \\
253.7\end{array}$ & $\begin{array}{l}0.0 \\
0.0\end{array}$ & $\begin{array}{l}0.0 \\
0.0\end{array}$ & $\begin{array}{l}4.4 \\
4.4\end{array}$ & $\begin{array}{l}241.7 \\
247.6\end{array}$ \\
\hline $03: 00$ & 6.5 & $25 \% .2$ & 0.0 & 0.0 & 4.4 & 252.2 \\
\hline $04: 00$ & 6.4 & 267.2 & 0.0 & 0.0 & 4.4 & 262.4 \\
\hline $05: 00$ & 6.2 & 273.0 & 0.0 & 0.0 & 4.2 & 266.7 \\
\hline $06: 00$ & 6.2 & 273.4 & 0.0 & 0.0 & 4.2 & 267.7 \\
\hline $07: 00$ & 6.1 & 274.4 & 0.0 & 0.0 & 4.0 & 263.9 \\
\hline $08: 00$ & 5.8 & 275.9 & 0.0 & 0.0 & 3.8 & 262.5 \\
\hline $09: 00$ & 5.6 & 270.3 & 0.0 & 0.0 & 3.7 & 257.5 \\
\hline $10: 00$ & 5.6 & 263.1 & 0.0 & 0.0 & 3.7 & 245.3 \\
\hline $11: 00$ & 5.8 & 235.5 & 0.0 & 0.0 & 3.9 & 223.4 \\
\hline $12: 00$ & 6.0 & 229.1 & 0.0 & 0.0 & 4.0 & 217.8 \\
\hline $13: 00$ & 6.3 & 223.7 & 0.0 & 0.0 & 4.1 & 213.5 \\
\hline $14: 00$ & 6.5 & 221.5 & 0.0 & 0.0 & 4.2 & 210.8 \\
\hline $15: 00$ & 6.7 & 222.5 & 0.0 & 0.0 & 4.3 & 210.5 \\
\hline $16: 00$ & 6.9 & 224.9 & 0.0 & 0.0 & 4.4 & 216.5 \\
\hline $17: 00$ & 7.0 & 227.1 & 0.0 & 0.0 & 4.5 & 214.1 \\
\hline $18: 00$ & 7.1 & 227.5 & 0.0 & 0.0 & 4.7 & 214.4 \\
\hline $19: 00$ & 7.2 & 225.1 & 0.0 & 0.0 & 4.8 & 215.1 \\
\hline $20: 00$ & 7.2 & 224.9 & 0.0 & 0.0 & 4.8 & 216.3 \\
\hline $21: 00$ & 7.1 & 227.8 & 0.0 & 0.0 & 4.7 & 218.9 \\
\hline $22: 00$ & 7.0 & $228 \cdot 1$ & 0.0 & 0.0 & 4.7 & 221.6 \\
\hline $23: 00$ & 6.7 & 234.3 & 0.0 & 0.0 & 4.5 & 228.3 \\
\hline
\end{tabular}


SITE ID:

SITE LOCATION: HOLYOKE, MA.

DATA :
JANIIARY I

1979 THROUGH DECEMBER

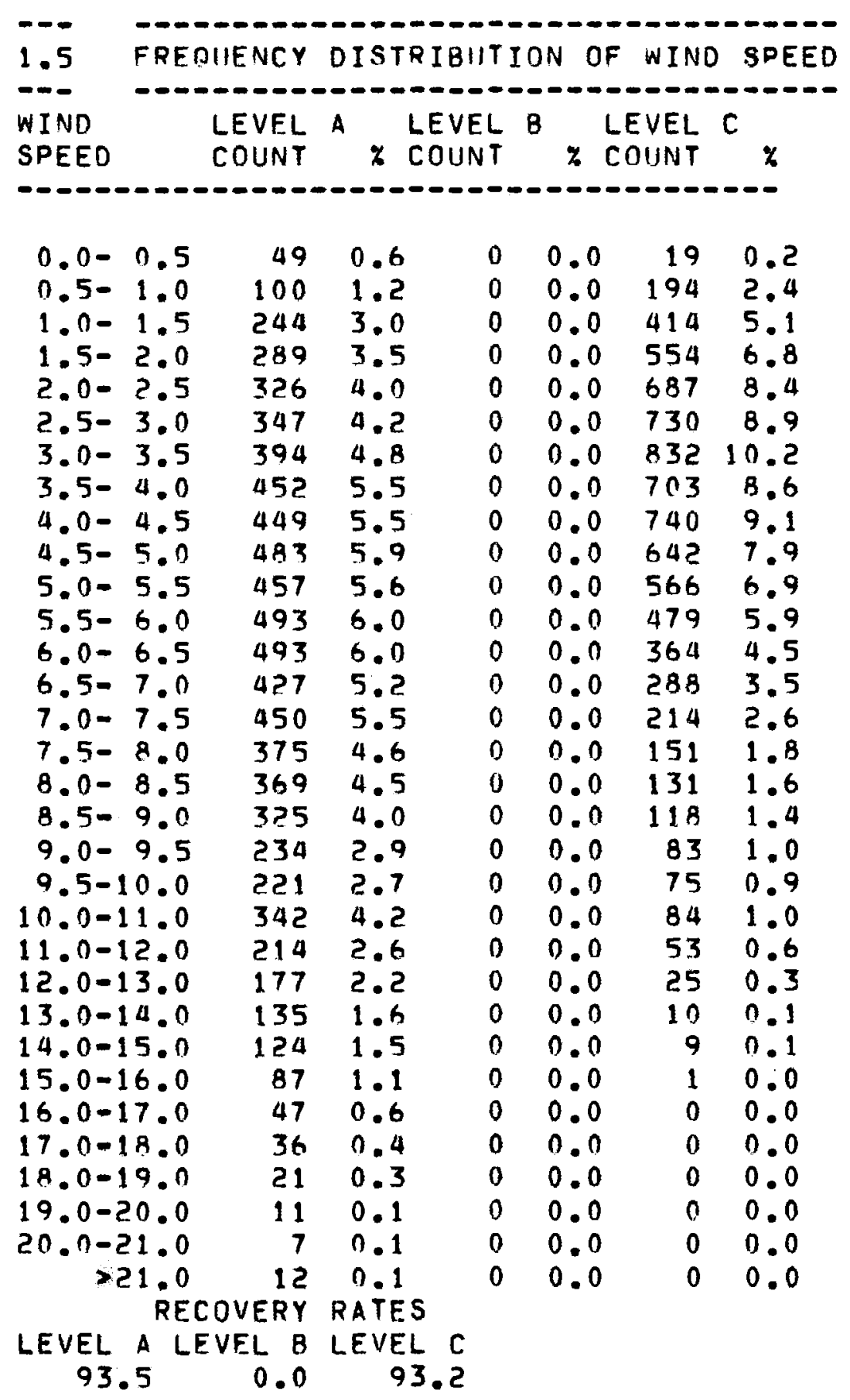


$\begin{array}{lllll}\text { SITE ID: } & \text { MA } & & \\ \text { SITE LOCATION: } & \text { HOLYOKE, MA, } & & \\ \text { DATA : } & \text { JANUARY } & \text { I979 THROUGH DECEMBER } & 1979\end{array}$

1.6 CUMULATIVE FREQUENCY DISTRIBUTION OF WIND SPEED

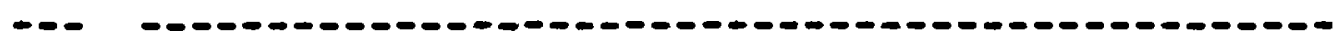

LEVEL A

\begin{tabular}{|c|c|c|}
\hline $\begin{array}{l}\text { WIND } \\
\text { SPEED }\end{array}$ & $\begin{array}{c}C F D \\
(A B S)\end{array}$ & $\begin{array}{l}C F D \\
(x)\end{array}$ \\
\hline$-\infty-\infty$ & $-\infty-\infty$ & $-\infty-\infty$ \\
\hline 0.5 & 49 & 0.6 \\
\hline 1.0 & 149 & 1.82 \\
\hline 1.5 & 393 & \\
\hline & 682 & 9.3 \\
\hline 2.5 & 1008 & 12.3 \\
\hline 3.0 & 1355 & \\
\hline 3.5 & 1749 & 21.36 \\
\hline 4.0 & 2201 & 26.87 \\
\hline 4.5 & 2650 & 32.36 \\
\hline 50 & 3133 & \\
\hline 5.5 & 3590 & 43.83 \\
\hline 6.0 & 4083 & \\
\hline & 4576 & 55.87 \\
\hline 7.0 & 5003 & 61.09 \\
\hline & 5453 & 66 \\
\hline & 5828 & 71.16 \\
\hline B. 5 & 6197 & \\
\hline 9. & 6522 & \\
\hline & 6756 & 8 \\
\hline 10. & 6977 & 19 \\
\hline 11.0 & 7319 & 89.37 \\
\hline 12.0 & 7533 & 91.98 \\
\hline 13 & 7710 & 94.14 \\
\hline 14 & 7845 & 95 \\
\hline 15 & 7969 & 97 \\
\hline 16.0 & 8056 & 98 \\
\hline 17.0 & 8103 & 98 \\
\hline & 8139 & \\
\hline & 8160 & \\
\hline & 8171 & \\
\hline & 8178 & 99 \\
\hline & 8190 & 100 \\
\hline
\end{tabular}

LEVEL 8

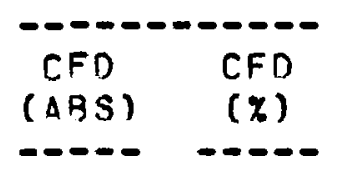

0.00

0.00

0.00

0.00

0.00

0.00

0.00

0.00

0.00

0.00

0.00

0.00

0.00

0.00

0.00

0.00

0.00

0.00

0.00

0.00

0.00

0.00

0.00

0.00

0.00

0.00

0.00

0.00

0.00

0.00

0.00

0.00
LEVEL C

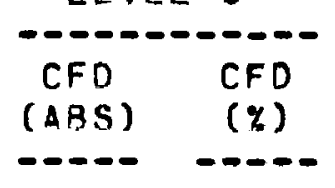

190.23

2132.61

$627 \quad 7.68$

$1181 \quad 14.46$

$1868 \quad 22.88$

$2598 \quad 31.81$

$3430 \quad 42.00$

413350.61

$4873 \quad 59.67$

$5515 \quad 67.54$

608174.47

$6560 \quad 80.33$

$6924 \quad 94.79$

$7212 \quad 88.32$

$7426 \quad 90.94$
7577

$\begin{array}{ll}7577 & 92.79\end{array}$

$\begin{array}{ll}7708 & 94.39\end{array}$

$7926 \quad 95.84$

$7909 \quad 96.85$

$\begin{array}{ll}7984 & 97.77\end{array}$

$8068 \quad 98.80$

812109.45

$8146 \quad 99.76$

$8156 \quad 99.88$

$8165 \quad 99.99$

8166100.00

$8166 \quad 100.00$

$8166 \quad 100.00$

$\$ 166100.00$

8166100.00

8166100.00

8166100.00 


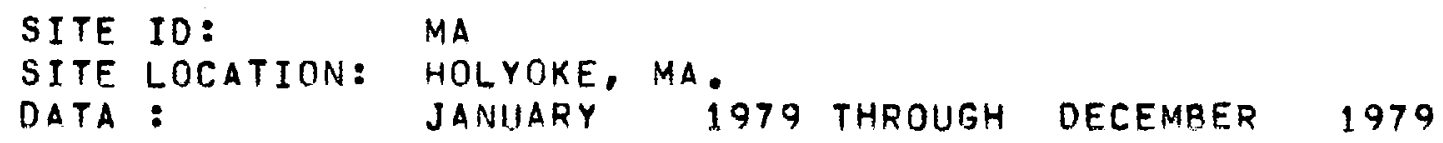

$\begin{array}{rrrrr}1 & 98 & 101 & 53 & 4 \\ ? & 56 & 55 & 32 & 0 \\ 3 & 34 & 35 & 12 & 0 \\ 4 & 24 & 28 & 15 & 1 \\ 5 & 18 & 25 & 3 & 1 \\ 6 & 18 & 26 & 4 & 1 \\ 7 & 11 & 15 & 2 & 0 \\ 8 & 19 & 15 & 5 & 0 \\ 9 & 8 & 18 & 3 & 0 \\ 10 & 16 & 12 & 1 & 0 \\ 11 & 13 & 12 & 5 & 0 \\ 12 & 6 & 10 & 1 & 0 \\ 13 & 7 & 16 & 1 & 0 \\ 14 & 5 & 16 & 1 & 0 \\ 15 & 1 & 8 & 1 & 0 \\ 16 & 6 & 10 & 0 & 0 \\ 17 & 3 & 7 & 1 & 0 \\ 18 & 3 & 5 & 0 & 0 \\ 19 & 4 & 9 & 0 & 0 \\ 20 & 2 & 7 & 1 & 0 \\ 21 & 3 & 9 & 0 & 0 \\ 22 & 3 & 6 & 0 & 0 \\ 23 & 1 & 3 & 1 & 0 \\ 23 & 8 & 57 & 4 & 0\end{array}$




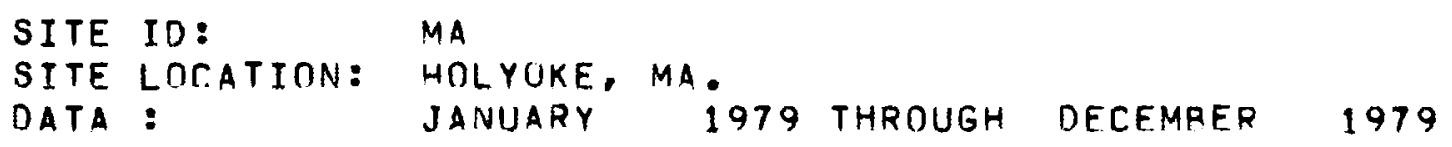


COUNT OF VIND SPEED VS. DIRECTION

ERCH LINE OF CHARACTERS REPAESENTS 40 OBSERVATIONS

SPEED INCREASES YITH DISTANCE FROM CENTER OF CIRCLES

ASSOCIATED RANGES IN MPS ARE $00-4 \quad 4-10 \quad 10-2020$ -

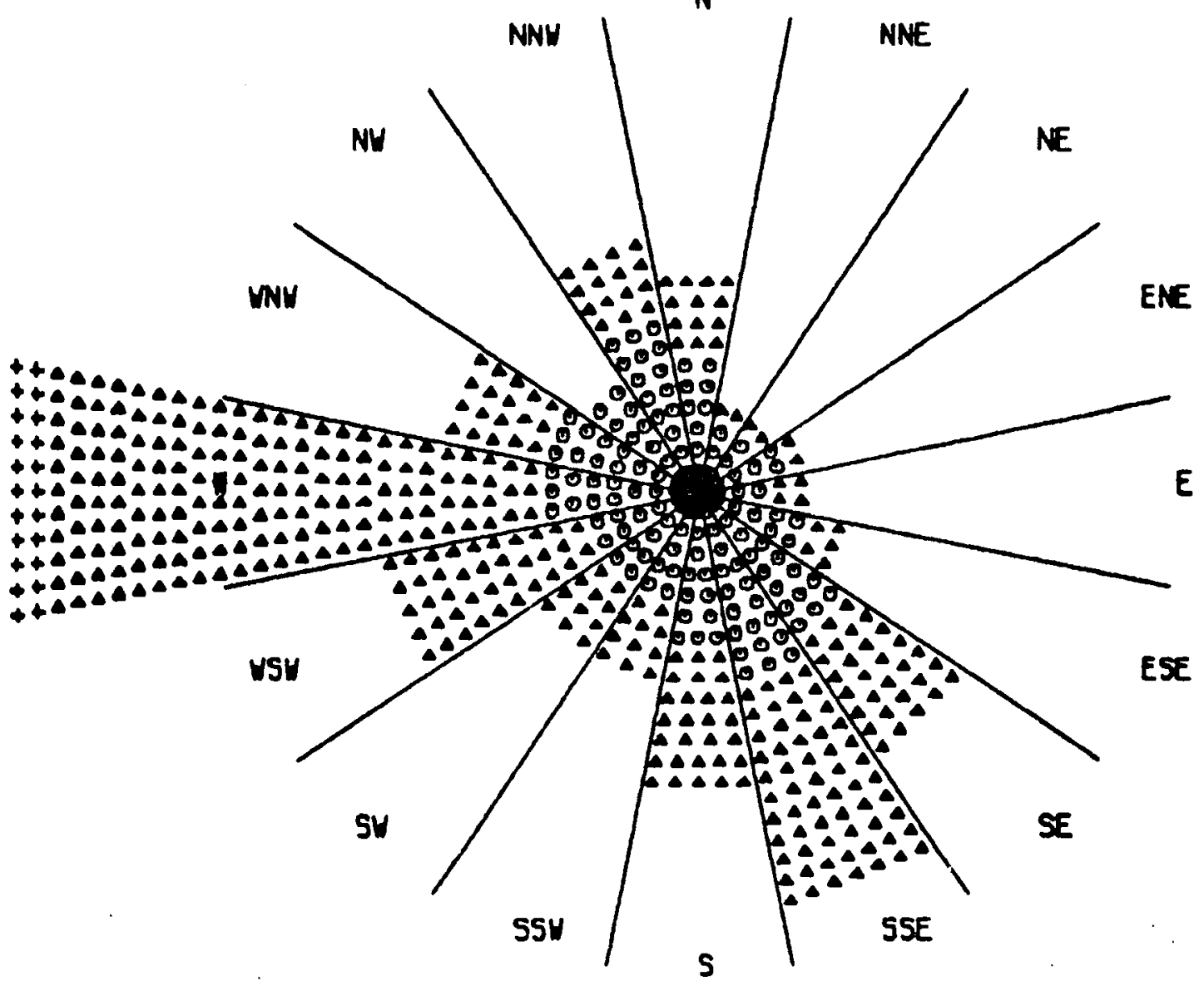

FIGURE 1. (MA) Wind Rose for Lowest Sensor Level 

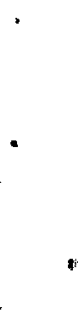

$-$
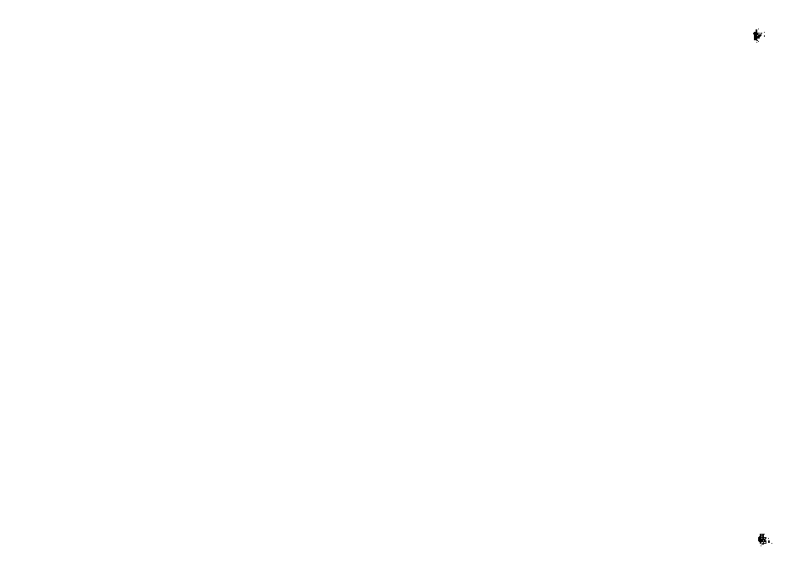
HURON, SOUTH DAKOTA 
$\begin{array}{llll}\text { SITE IO: } & \text { SO } & & \\ \text { SITE LOCATION: } & \text { HURON, SU. } & & \\ \text { DATA : } & \text { JANUARY } 1979 \text { THROUGH DECEMBER } & 1979\end{array}$

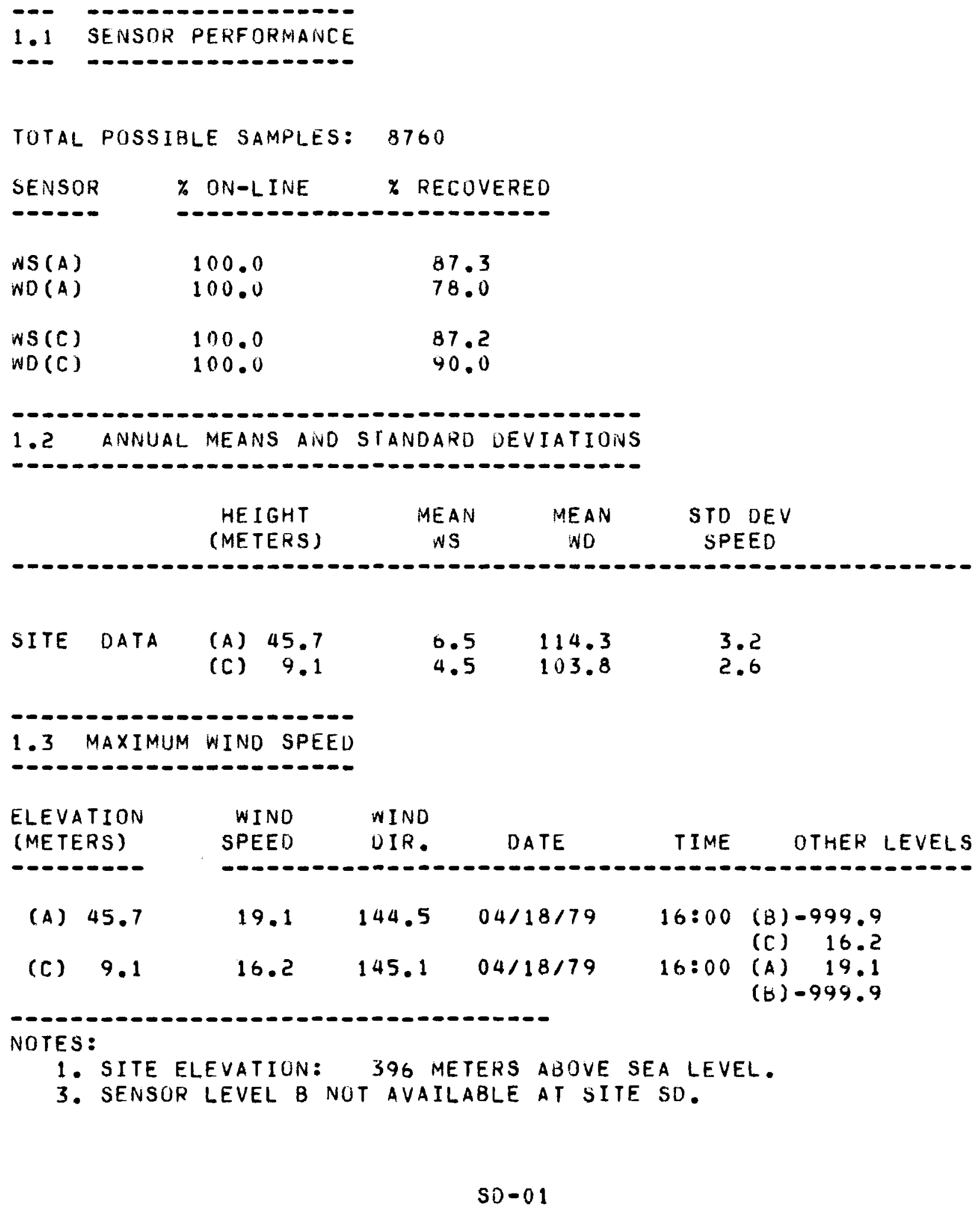


SITE IO: SD

SITE LUCATION: HURON, SU.

DATA:

JANUARY

1979 THROUGH DECEMBER 1979

1.4 NIND SFEED AIVU DIKECTIUN VERSUS PIME-UF-DAY

\begin{tabular}{|c|c|c|c|c|c|c|}
\hline \multirow{2}{*}{$\begin{array}{l}\text { TIME } \\
\text { UF DAY } \\
--0--\infty\end{array}$} & ws & $\begin{array}{rl}E L & A \\
& \text { WD }\end{array}$ & wS & $\frac{B}{\text { WO }}$ & NS & $\begin{array}{l}\text { EL C } \\
\text { WU }\end{array}$ \\
\hline & & & & -. & & $--\infty$ \\
\hline $00: 00$ & 6.6 & 109.8 & 0.0 & 0.0 & 4.1 & 113.4 \\
\hline $01: 00$ & 6.6 & 117.0 & 0.0 & 0.0 & 4.0 & 122.0 \\
\hline $02: 00$ & 6.5 & 120.4 & 0.0 & 0.0 & 4.0 & 115.7 \\
\hline $03: 00$ & 0.5 & 145.8 & 0.0 & 0.0 & 4.0 & 125.5 \\
\hline $04: 00$ & 6.3 & 145.2 & 0.0 & 0.0 & 4.0 & 127.6 \\
\hline $05: 00$ & 6.3 & 137.6 & 0.0 & 0.0 & 3.9 & 117.6 \\
\hline $06: 00$ & 6.3 & 143.8 & 0.0 & 0.0 & 3.9 & 115.4 \\
\hline $07: 00$ & 6.0 & 150.6 & 0.0 & 0.0 & 3.9 & 112.3 \\
\hline $08: 00$ & 5.9 & 132.0 & 0.0 & 0.0 & 4.2 & 121.8 \\
\hline $09: 00$ & 6.0 & 141.0 & 0.0 & 0.0 & 4.6 & 130.3 \\
\hline $10: 00$ & 6.1 & 210.0 & 0.0 & 0.0 & 4.9 & 155.5 \\
\hline $11: 00$ & 6.3 & 192.2 & 0.0 & 0.0 & 5.1 & 153.0 \\
\hline $12: 00$ & 6.5 & 260.3 & 0.0 & 0.0 & 5.3 & 240.9 \\
\hline $13: 00$ & 6.8 & 269.0 & 0.0 & 0.0 & 5.5 & 250.9 \\
\hline $14: 00$ & 6.8 & 301.9 & 0.0 & 0.0 & 5.5 & 270.9 \\
\hline $15: 00$ & 6.8 & 285.2 & 0.0 & 0.0 & 5.4 & 283.1 \\
\hline $16: 00$ & 6.7 & 299.5 & 0.0 & 0.0 & 5.2 & 291.7 \\
\hline $17: 00$ & 6.7 & 320.1 & 0.0 & 0.0 & 5.0 & 348.0 \\
\hline $18: 00$ & 6.7 & 1.7 & 0.0 & 0.0 & 4.7 & 32.0 \\
\hline $19: 00$ & 6.4 & 16.1 & 0.0 & 0.0 & 4.3 & 44.2 \\
\hline $20: 00$ & 6.3 & 47.1 & 0.0 & 0.0 & 4.0 & 70.1 \\
\hline $21: 00$ & 6.4 & 77.1 & 0.0 & 0.0 & 3.9 & 81.1 \\
\hline $22: 00$ & 6.7 & 88.3 & 0.0 & 0.0 & 4.1 & 85.5 \\
\hline $23: 00$ & 6.7 & 97.7 & 0.0 & 0.0 & 4.1 & 103.4 \\
\hline
\end{tabular}


SITE ID: $\quad$ SD

SITE LOCATION: HURON, SO.

JANUARY

1979 THROUGH DECEMBER

1979

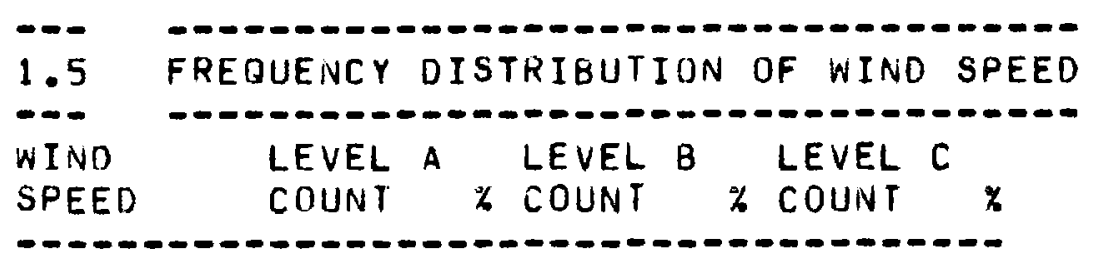

$\begin{array}{rrrrrrr}0.0-0.5 & 30 & 0.4 & 0 & 0.0 & 74 & 1.0 \\ 0.5-1.0 & 91 & 1.2 & 0 & 0.0 & 208 & 2.7 \\ 1.0-1.5 & 161 & 2.1 & 0 & 0.0 & 412 & 5.4 \\ 1.5-2.0 & 217 & 2.8 & 0 & 0.0 & 592 & 7.7 \\ 2.0-2.5 & 273 & 3.6 & 0 & 0.0 & 657 & 8.6 \\ 2.5-3.0 & 297 & 3.9 & 0 & 0.0 & 735 & 9.6 \\ 3.0-3.5 & 349 & 4.6 & 0 & 0.0 & 636 & 8.3 \\ 3.5-4.0 & 426 & 5.6 & 0 & 0.0 & 544 & 7.1 \\ 4.0-4.5 & 454 & 5.9 & 0 & 0.0 & 584 & 7.6 \\ 4.5-5.0 & 440 & 5.8 & 0 & 0.0 & 469 & 6.1 \\ 5.0-5.5 & 466 & 6.1 & 0 & 0.0 & 383 & 5.0 \\ 5.5-6.0 & 445 & 5.8 & 0 & 0.0 & 384 & 5.0 \\ 6.0-6.5 & 527 & 6.9 & 0 & 0.0 & 337 & 4.4 \\ 6.5-7.0 & 450 & 5.9 & 0 & 0.0 & 278 & 3.6 \\ 7.0-7.5 & 385 & 5.0 & 0 & 0.0 & 262 & 3.4 \\ 7.5-8.0 & 381 & 5.0 & 0 & 0.0 & 225 & 2.9 \\ 8.0-8.5 & 359 & 4.7 & 0 & 0.0 & 199 & 2.6 \\ 8.5-9.0 & 292 & 3.8 & 0 & 0.0 & 155 & 2.0 \\ 9.0-9.5 & 309 & 4.0 & 0 & 0.0 & 126 & 1.6 \\ 9.5-10.0 & 265 & 3.5 & 0 & 0.0 & 103 & 1.3 \\ 10.0-11.0 & 379 & 5.0 & 0 & 0.0 & 136 & 1.8 \\ 11.0-12.0 & 241 & 3.2 & 0 & 0.0 & 78 & 1.0 \\ 12.0-13.0 & 151 & 2.0 & 0 & 0.0 & 44 & 0.6 \\ 13.0-14.0 & 104 & 1.4 & 0 & 0.0 & 17 & 0.2 \\ 14.0-15.0 & 69 & 0.9 & 0 & 0.0 & 3 & 0.0 \\ 15.0-16.0 & 41 & 0.5 & 0 & 0.0 & 0 & 0.0 \\ 16.0-17.0 & 29 & 0.4 & 0 & 0.0 & 1 & 0.0 \\ 17.0-18.0 & 15 & 0.2 & 0 & 0.0 & 0 & 0.0 \\ 18.0-19.0 & 2 & 0.0 & 0 & 0.0 & 0 & 0.0 \\ 19.0-20.0 & 1 & 0.0 & 0 & 0.0 & 0 & 0.0 \\ 20.0-21.0 & 0 & 0.0 & 0 & 0.0 & 0 & 0.0 \\ >21.0 & 0 & 0.0 & 0 & 0.0 & 0 & 0.0\end{array}$

LEVEL A LEVEL B LEVEL C
87. 3
0.0
87.2 
SITE ID:

SO

SITE LOCATION: HURON, SU.

DATA : JANUARY

1979 THROUGH DECEMBER

1979

1.6

CUMULATIVE FREQUENCY DISTRIBUTIUN OF NIND SPEED

\begin{tabular}{|c|c|c|}
\hline & LEV & L A \\
\hline $\begin{array}{l}\text { WIND } \\
\text { SPEED }\end{array}$ & $\begin{array}{c}C F U \\
(\triangle B S)\end{array}$ & $\begin{array}{l}C F D \\
(\%)\end{array}$ \\
\hline$---\infty-$ & $--\infty$ & 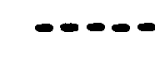 \\
\hline 0.5 & 30 & 0.39 \\
\hline 1.0 & 121 & 1.58 \\
\hline 1.5 & 282 & 3.69 \\
\hline 2.0 & 499 & 6.52 \\
\hline 2.5 & 772 & 10.09 \\
\hline 3.0 & 1069 & 13.98 \\
\hline 3.5 & 1418 & 18.54 \\
\hline 4.0 & 1844 & 24.11 \\
\hline 4.5 & 2298 & 30.04 \\
\hline 5.0 & 2738 & 35.80 \\
\hline 5.5 & 3204 & 41.89 \\
\hline 6.0 & 3649 & 47.71 \\
\hline 6.5 & 4176 & 54.60 \\
\hline 7.0 & 4626 & 60.48 \\
\hline 7.5 & 5011 & 65.51 \\
\hline 8.0 & 5392 & 70.49 \\
\hline 8.5 & 5751 & 75.19 \\
\hline 9.0 & 6043 & 79.00 \\
\hline 9.5 & 6352 & 83.04 \\
\hline 10.0 & 6617 & 86.51 \\
\hline 11.0 & 6996 & 91.46 \\
\hline 12.0 & 7237 & 94.61 \\
\hline 13.0 & 7388 & 96.59 \\
\hline 14.0 & 7492 & 97.95 \\
\hline 15.0 & 7561 & 98.85 \\
\hline 16.0 & 7602 & 99.39 \\
\hline 17.0 & 7631 & 99.76 \\
\hline 18.0 & 7646 & 99.96 \\
\hline 19.0 & 7648 & 99.99 \\
\hline 20.0 & 7649 & 100.00 \\
\hline 21.0 & 7649 & 100.00 \\
\hline-21.0 & 7649 & 100.00 \\
\hline
\end{tabular}

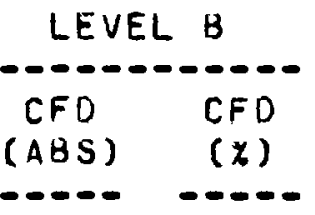

0.00

0.00

0.00

0.00

0.00

0.00

0.00

0.00

0.00

0.00

0.00

0.00

0.00

0.00

0.00

0.00

0.00

0.00

0.00

0.00

0.00

0.00

0.00

0.00

0.00

0.00

0.00

0.00

0.00

0.00

0.00

0.00
LEVEL C

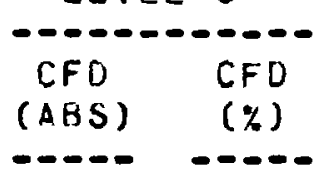

$\begin{array}{ll}74 & 0.97\end{array}$

$282 \quad 3.69$

6949.08

$1286 \quad 16.83$

194325.43

$2678 \quad 35.04$

$\begin{array}{ll}3314 & 43.37\end{array}$

$3858 \quad 50.48$

$4442 \quad 58.13$

491164.26

$5294 \quad 69.28$

$5678 \quad 74.30$

$6015 \quad 78.71$

$6293 \quad 82.35$

$6555 \quad 85.78$

$6780 \quad 8 B .72$

697991.32

713493.35

$7260 \quad 95.00$

736396.35

$7499 \quad 98.13$

$7577 \quad 99.15$

762199.73

763899.95

764199.99

764199.99

7642100.00

7642100.00

7642100.00

7642100.00

7642100.00

7642100.00 
$\begin{array}{ll}\text { SITE ID: } & \text { SO } \\ \text { SITE LOCATION: } & \text { HURON, SO. }\end{array}$

DATA

JAIVUARY

1979 THROIUGH

DECEMRER 1979

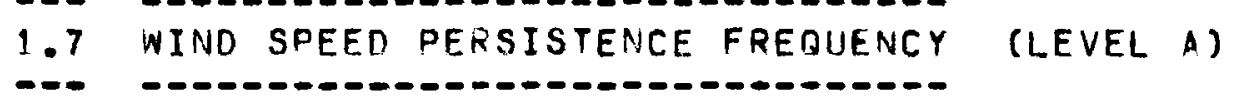

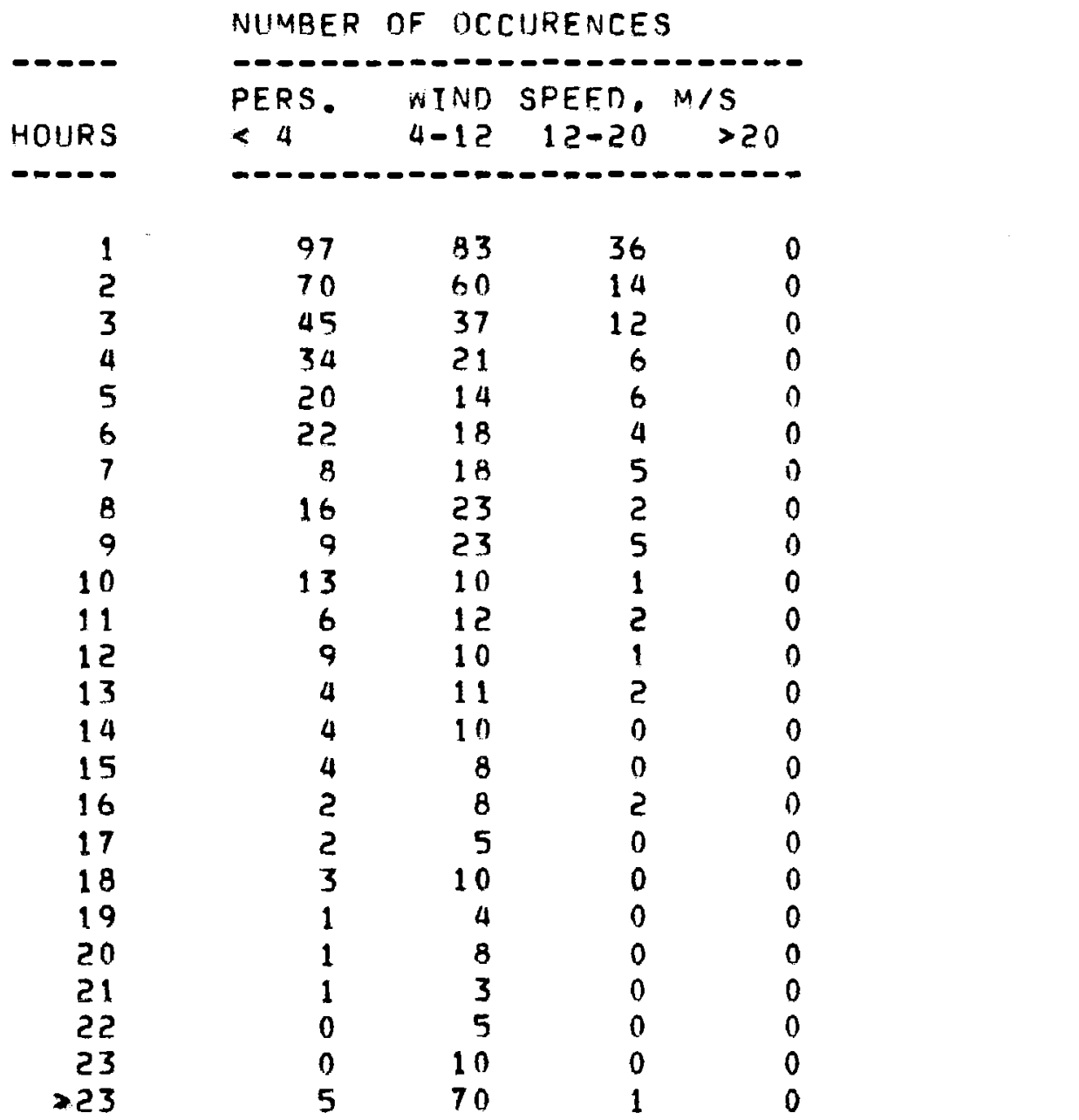


SITE ID:

SITE LOCATION:

DATA :
SD

HIJRON, SD. JANUARY

1.8

POWER LAW EXPONENT AND WID DISTRIBUTION

$--$

POWER LAW EXPONENT AND WUD DISTR

WIND DTR

(ELEV A) ALPHA ALPHA ALPHA ALPHA

$(A, B)(B, C)(A, C)(A, B, C) \quad \nVdash A \quad \neq B \quad \times C$

$-\cdot-\cdot-\cdot-$

$\begin{array}{lllllllr}\text { N } & 0.00 & 0.00 & 0.24 & 0.00 & 3.87 & 0.00 & 4.46 \\ \text { NNE } & 0.00 & 0.00 & 0.22 & 0.00 & 2.73 & 0.00 & 2.89 \\ \text { NE } & 0.00 & 0.00 & 0.25 & 0.00 & 2.42 & 0.00 & 2.41 \\ \text { ENE } & 0.00 & 0.00 & 0.23 & 0.00 & 3.03 & 0.00 & 3.25 \\ \text { E } & 0.00 & 0.00 & 0.22 & 0.00 & 4.48 & 0.00 & 5.19 \\ \text { ESE } & 0.00 & 0.00 & 0.23 & 0.00 & 7.36 & 0.00 & 8.69 \\ \text { SE } & 0.00 & 0.00 & 0.27 & 0.00 & 14.73 & 0.00 & 15.61 \\ \text { SSE } & 0.00 & 0.00 & 0.17 & 0.00 & 8.07 & 0.00 & 8.44 \\ \text { S } & 0.00 & 0.00 & 0.21 & 0.00 & 3.56 & 0.00 & 3.36 \\ \text { SSW } & 0.00 & 0.00 & 0.24 & 0.00 & 1.73 & 0.00 & 1.69 \\ \text { SN } & 0.00 & 0.00 & 0.25 & 0.00 & 2.04 & 0.00 & 2.67 \\ \text { WSW } & 0.00 & 0.00 & 0.30 & 0.00 & 2.88 & 0.00 & 3.27 \\ \text { W } & 0.00 & 0.00 & 0.26 & 0.00 & 5.24 & 0.00 & 4.87 \\ \text { WNW } & 0.00 & 0.00 & 0.26 & 0.00 & 7.92 & 0.00 & 8.09 \\ \text { NW } & 0.00 & 0.00 & 0.20 & 0.00 & 8.93 & 0.00 & 10.32 \\ \text { NNW } & 0.00 & 0.00 & 0.16 & 0.00 & 8.84 & 0.00 & 10.15\end{array}$

NOTES:

$$
\text { 1. } \frac{W S(U P)}{W S(L O)}=\frac{Z(U P)}{Z(L O)}
$$

LOG (WS (UP)/WS (LO))

2. $A L P H A=\ldots$

$\operatorname{LOG}(Z(U P) / Z(L))$
WHERE; $\quad Z=E L E V A T I O N$

WS =WINO SPEED 
COUNT OF VIND SPEED VS. DIRECTION

EACH LINE OF CHAAACTERS REPRESENTS 40 OBSERVATIONS

SPEED INCREASES WITH DISTANCE FROM CENTER OF CIRCLES

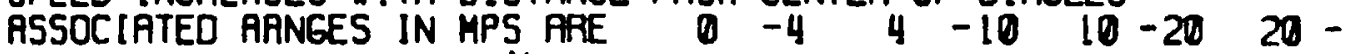

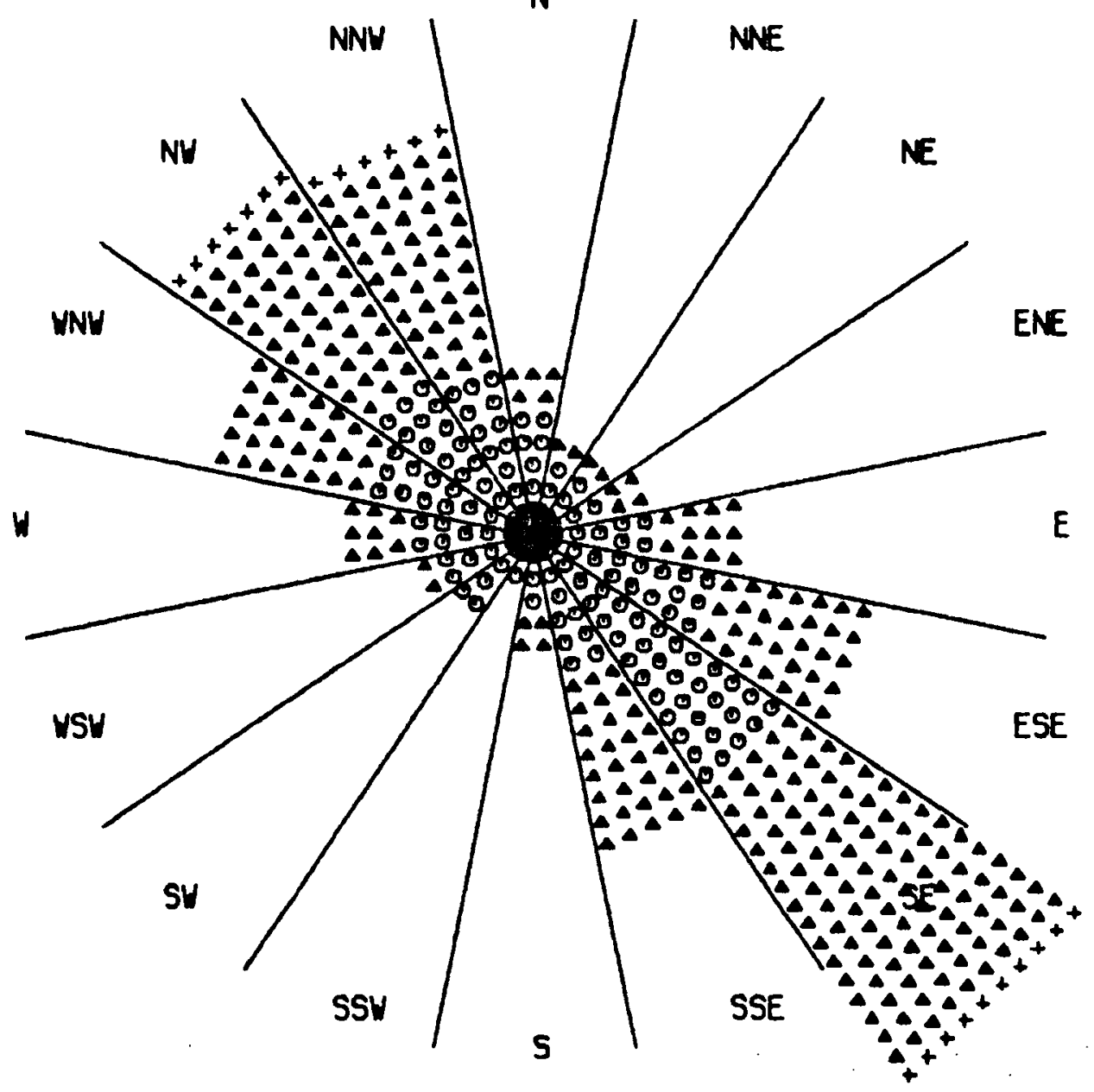

FIGURE 1. (SD) Wind Rose for Lowest Sensor Level 
: 
KINGSLEY DAM, NEBRASKA 


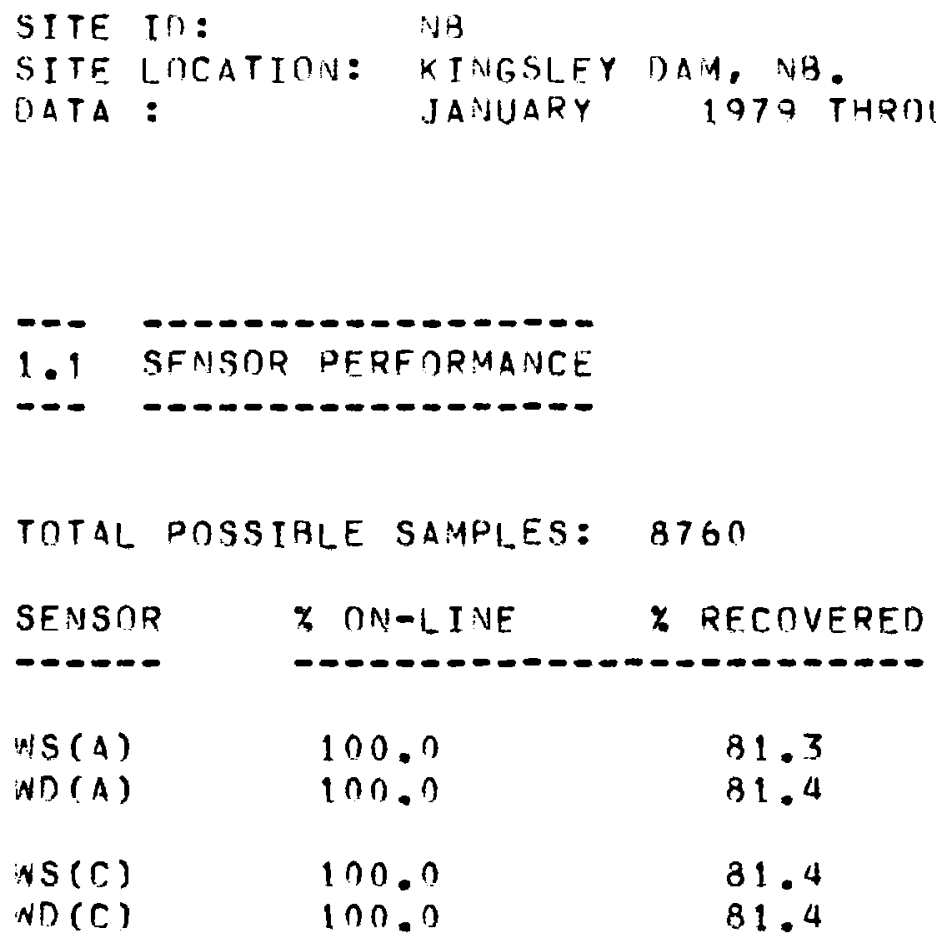

1.2 ANNUAL MFANS AND STAMDART DEVIATIONS

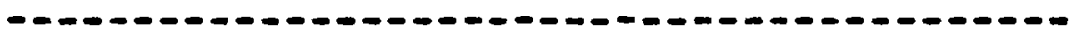

$\begin{array}{cccc}\text { HFIGHT MEAN } & \text { MEAN } & \text { STD DEV } \\ \text { (MFTERS) } & \text { WS } & \text { ND } & \text { SPEEO }\end{array}$

$\begin{array}{rrrrrr}\text { SITE DATA } & \text { (A) } & 45.7 & 6.2 & 274.4 & 3.1 \\ & \text { (C) } & 9.1 & 4.8 & 278.4 & 2.5\end{array}$

\subsection{MAXIMIJM WTNO SPEF.D}

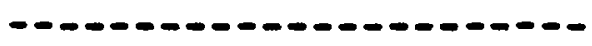

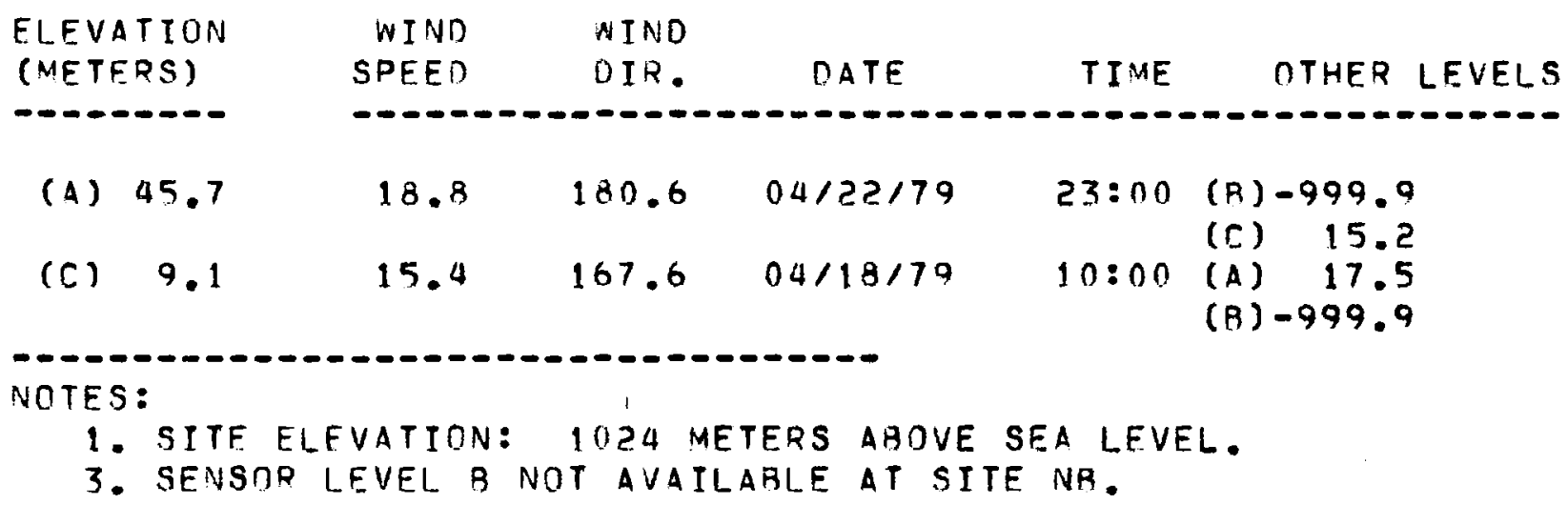


$\begin{array}{ll}\text { SITE ID: } & \text { MA } \\ \text { SITE LICATION: KTMGSLEY DAM, HB. }\end{array}$

DATA : JANUARY 1979 THROUJFH DECFMBER 1979

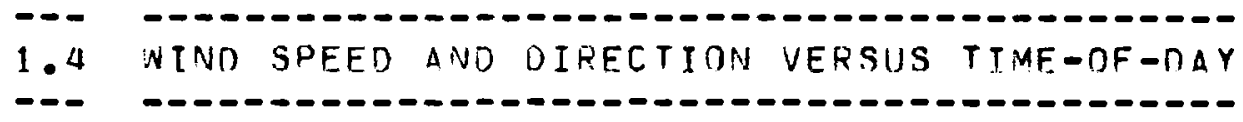

\begin{tabular}{|c|c|c|c|c|c|c|}
\hline TIME & & EL A & & $\begin{array}{l}B \\
---\end{array}$ & & 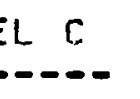 \\
\hline OF חAY & NS & ND & WS & WD & NS & Wo \\
\hline------ & - & $=--$ & $-\infty$ & & & ---- \\
\hline $00: 00$ & 6.6 & 193.8 & 0.0 & 0.0 & 4.7 & 193.8 \\
\hline $01=00$ & 6.5 & 248.4 & 0.0 & 0.0 & 4.7 & 252.6 \\
\hline $02: 00$ & 6.3 & 253.7 & 0.0 & 0.0 & 4.6 & 249.8 \\
\hline $03: 00$ & 6.1 & 266.3 & 0.0 & 0.0 & 4.4 & 270.4 \\
\hline $04: 00$ & 5.9 & 272.1 & 0.0 & 0.0 & 4.3 & 274.2 \\
\hline $05: 00$ & 5.8 & 274.1 & 0.0 & 0.0 & 4.2 & 265.4 \\
\hline $06: 00$ & 5.7 & 268.2 & 0.0 & 0.0 & 4.2 & 259.1 \\
\hline $07: 00$ & 5.7 & 274.1 & 0.0 & 0.0 & 4.4 & 276.2 \\
\hline $08: 0 n$ & 5.6 & 280.5 & 0.0 & 0.0 & 4.7 & 289.3 \\
\hline $09: 00$ & 5.7 & 279.8 & 0.0 & 0.0 & 4.9 & 288.9 \\
\hline $10: 00$ & 5.0 & 288.1 & 0.0 & 0.0 & 5.0 & 299.1 \\
\hline $11: n n$ & 6.0 & 289.1 & 0.0 & 0.0 & 5.1 & 299.4 \\
\hline $1 ?: 00$ & 6.1 & 290.8 & 0.0 & 0.0 & 5.2 & 300.1 \\
\hline $13: 00$ & 6.2 & 288.6 & 0.0 & 0.0 & 5.3 & 296.6 \\
\hline $14: 00$ & 6.2 & 291.6 & 0.0 & 0.0 & 5.3 & 299.3 \\
\hline $15: 00$ & 6.4 & 297.4 & 0.0 & 0.0 & 5.3 & 310.5 \\
\hline $16: 00$ & 6.4 & 330.5 & 0.0 & 0.0 & 5.1 & 347.6 \\
\hline $17: 00$ & h.2 & 27.7 & 0.0 & 0.0 & 4.8 & 44.7 \\
\hline $18: 00$ & 6.2 & 81.4 & 0.0 & 0.0 & 4.5 & 98.1 \\
\hline $19: 00$ & 6.4 & 100.6 & 0.0 & 0.0 & 4.5 & 118.0 \\
\hline $20: 00$ & 6.6 & $1 \cap 6.3$ & 0.0 & 0.0 & 4.6 & 118.6 \\
\hline $21: 00$ & 6.8 & 126.6 & 0.0 & 0.0 & 4.8 & 139.1 \\
\hline $22=00$ & 6.7 & 151.7 & 0.0 & 0.0 & 4.8 & 152.7 \\
\hline $23: 00$ & 6.7 & 168.6 & 0.0 & 0.0 & 4.8 & 162.8 \\
\hline
\end{tabular}


STTE TO: NB

SITE l.OCATION: KIMGSLEY DAM, NB.

DATA : JANUIARY 1979 THROUGH DECEMEER 1979

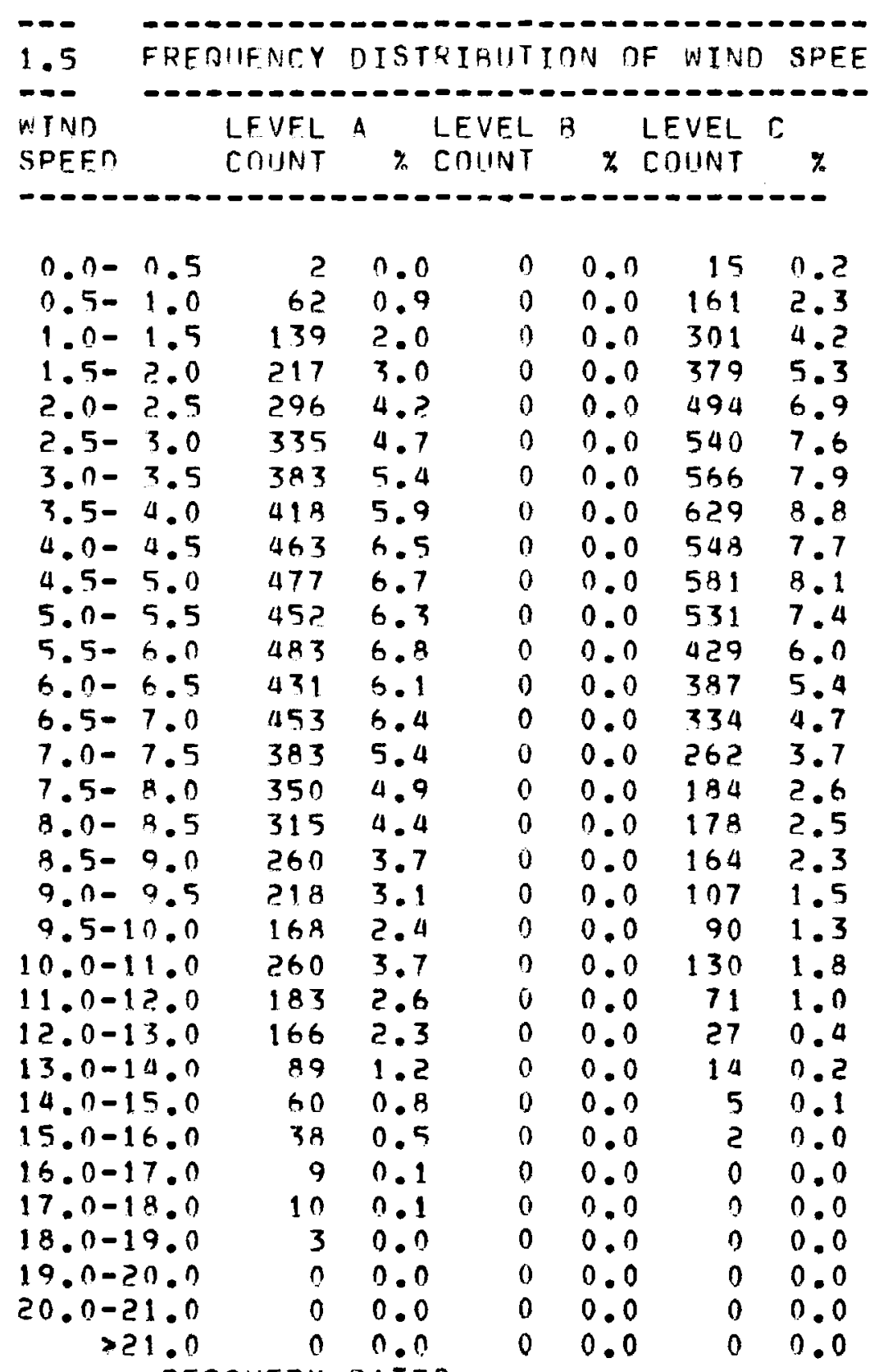

LEVEL A LEVFL B LEVEL C
81.3
0.0
B 1.4 

SITE ID:
NR
SITE LOCATION: KINGSLEY DAM, NB.

- - - -

NIAID

SPEED

-O--

0.5

1.0

1.5

2. 0

2.5

3.0

3.5

4.0

4.5

5.0

5.5

6.0

6.5

7.0

7.5

8.0

8.5

9.0

9.5

10.0

11.0

12.0

13.0

14.0

15.0

16.0

17.0

18.0

19.0

20.0

21.0

$>21.0$
LEVEL A

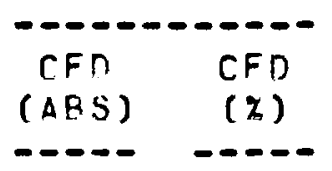

2

64

203

$42 n$

796

$\ln 51$

1434

$185 ?$

2315

279?

3244

$37 ? 7$

4158

4611

4994

5344

5650

5919

6) 37

6305

6565

6748

6914

7003

7063

7101

7110

7120

7123

7123

7123

7123

\subsection{3}

0.90

2.85

5.90

10.05

14.76

20.13

26.00

32.50

$39 . ? 0$

45.54

52.32

58.37

44.73

70.11

75.02

79.45

83.10

86.16

ค月. 52 ?

92.17

94.74

97.07

98.3 ?

99.16

09.69

99.82

99.96

100.00

100.00

100.00

100.00

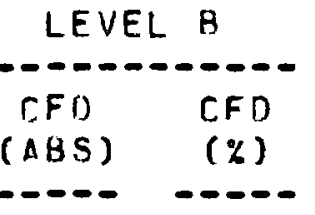

\section{0}

0

0

ก

0

0

0

0

0

0

0.00

0.00

0.00

$0.0 n$

0.00

0.00

0.00

0.00

0.00

0.00

0.00

0.00

0.00

0.00

0.00

0.00

0.00

0.00

0.00

0.00

0.00

0.00

$0.0 n$

0.00

0.00

0.00

0.00

0.00

0.00

0.00

0.00

0.00
LFVEL C

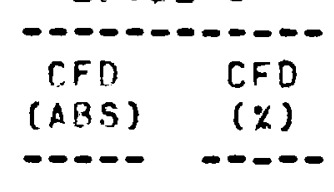

$\begin{array}{rr}15 & 0.21 \\ 176 & 2.47 \\ 477 & 6.69\end{array}$

$856 \quad 12.01$

$\begin{array}{lll}1350 & 18.94\end{array}$

$1890 \quad 26.51$

$2456 \quad 34.45$

$3045 \quad 43.27$

363350.96

$4214 \quad 59.11$

$4745 \quad 66.56$

517472.58

556178.01

$5805 \quad 82.69$

$6157 \quad 86.37$

634188.95

$6519 \quad 91.44$

669303.70

$6790 \quad 95.24$

6820 96.51

$7010 \quad 98.33$

708199.33

$71 \cap 8 \quad 99.71$

$7122 \quad 99.90$

$7127 \quad 99.97$

7129100.00

7129100.00

7129100.00

7129100.00

7129100.00

7129100.00

7129100.00 
SITE. ID:

SITE LOCATION: KINGSLEY DAM, NB.

DATA :
NH

ITANIIARY

1.7

WIND SPEED PERSISTFNCE FREQUENCY

(LEVEL A)

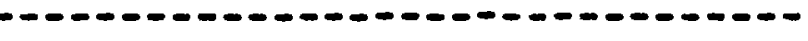

NIIIMBER DF OCCURENCES

\begin{tabular}{|c|c|c|c|c|}
\hline HOIIRS & $\begin{array}{l}\text { PERS. } \\
<4\end{array}$ & $\begin{array}{l}\text { NND } \\
4-12\end{array}$ & $\begin{array}{r}\text { SPEED, } \\
12-20\end{array}$ & $\begin{array}{l}M / S \\
>20\end{array}$ \\
\hline$-\cdots$ & $---\infty$ & 8 & $-\infty$ & - - - \\
\hline 1 & 120 & 120 & 47 & 0 \\
\hline$?$ & 86 & 64 & 22 & 0 \\
\hline 3 & 50 & 49 & 15 & n \\
\hline 4 & 37 & 44 & 7 & 0 \\
\hline 5 & 27 & 35 & 4 & 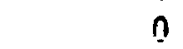 \\
\hline 6 & 36 & 22 & 3 & 0 \\
\hline 7 & 18 & 15 & 2 & 0 \\
\hline 8 & 9 & ? 5 & 4 & 0 \\
\hline 0 & 14 & 18 & 4 & 0 \\
\hline 10 & 13 & 21 & $n$ & 0 \\
\hline 11 & 4 & 14 & 3 & 0 \\
\hline 12 & 6 & 9 & 1 & 0 \\
\hline 13 & 4 & 16 & 0 & 0 \\
\hline 14 & 1 & 9 & 1 & 0 \\
\hline 15 & 4 & 11 & 1 & 0 \\
\hline 16 & 1 & 7 & 0 & 0 \\
\hline 17 & 3 & 9 & 1 & 0 \\
\hline $1 A$ & 0 & 9 & 0 & 0 \\
\hline 19 & 1 & 10 & 0 & 0 \\
\hline 20 & 0 & 5 & 0 & 0 \\
\hline 21 & 1 & 6 & 0 & 0 \\
\hline ?2 & 1 & 7 & 0 & 0 \\
\hline 23 & 1 & 3 & 0 & 0 \\
\hline$>2.3$ & 2 & 42 & 0 & 0 \\
\hline
\end{tabular}




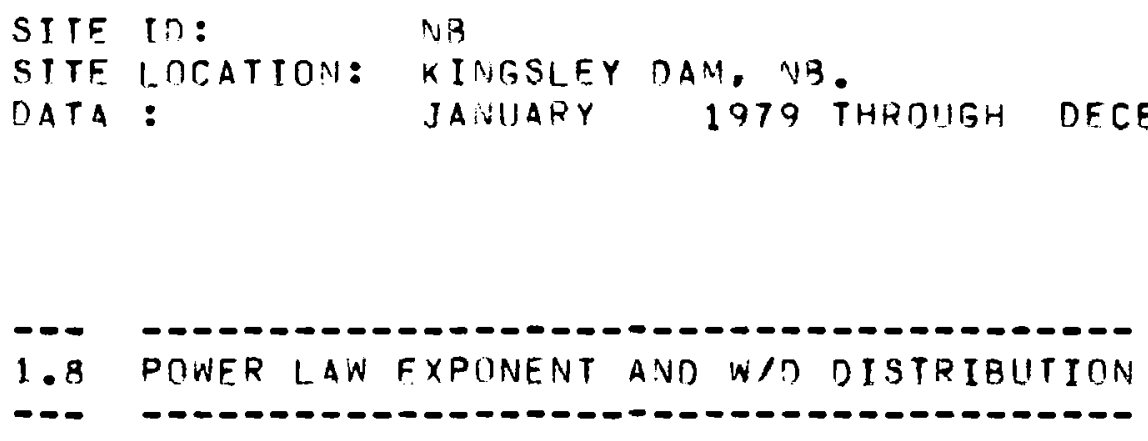

NTYN DIR

\begin{tabular}{|c|c|c|c|c|c|c|c|}
\hline$(E L F V$ A $)$ & $\begin{array}{l}A L P H A \\
(A, B)\end{array}$ & $\begin{array}{l}A L P H A \\
(R, C)\end{array}$ & $\begin{array}{l}A L P H A \\
(A, C)\end{array}$ & $\begin{array}{l}A L P H A \\
(A, B, C)\end{array}$ & $\because A$ & $\% \mathrm{P}$ & $\% \mathrm{C}$ \\
\hline-9 & & & & & & & \\
\hline N & 0.00 & 0.00 & 0.18 & 0.00 & 6.19 & 0.00 & 5.92 \\
\hline NINE. & 0.00 & 0.00 & 0.15 & 0.00 & 4.82 & 0.00 & 5.57 \\
\hline NF & $n .00$ & 0.00 & 0.08 & 0.00 & 4.89 & 0.00 & 5.16 \\
\hline ENE & 0.00 & 0.00 & 0.11 & 0.00 & 5.55 & 0.00 & 5.62 \\
\hline$E$ & ח. & $0.0 n$ & 0.17 & 0.00 & 4.80 & 0.00 & 5.84 \\
\hline ESE & 0.00 & 0.00 & n. 23 & 0.00 & 4.25 & 0.00 & 4.73 \\
\hline SE & 0.00 & 0.00 & 0.21 & 0.00 & 5.53 & 0.00 & 4.97 \\
\hline S.SE & 0.00 & 0.00 & 0.20 & 0.00 & 8.25 & 0.00 & 6.65 \\
\hline$S$ & $n .0 n$ & 0.00 & 0.14 & 0.00 & 8.31 & 0.00 & 7.98 \\
\hline SSW & $n . n n$ & 0.00 & 0.12 & 0.00 & 5.53 & 0.00 & 6.52 \\
\hline SW & 0.00 & 0.00 & 0.08 & 0.00 & 3.80 & 0.00 & 3.62 \\
\hline NSW & 0.00 & 0.00 & 0.19 & 0.00 & 4.31 & 0.00 & 5.29 \\
\hline A & 0.00 & 0.00 & 0.21 & 0.00 & 7.60 & 0.00 & 7.97 \\
\hline$N N N$ & 0.00 & 0.00 & 0.15 & 0.00 & 12.78 & 0.00 & 11.56 \\
\hline$N W$ & 0.00 & 0.00 & 0.16 & 0.00 & 6.94 & 0.00 & 7.45 \\
\hline NNW & 0.00 & 0.00 & 0.18 & 0.00 & 6.44 & 0.00 & 5.15 \\
\hline
\end{tabular}

NOTES:

$$
\begin{aligned}
& \text { WS(UP) ALPHA } \\
& \text { 1. } \frac{\text { WS (UP) }}{S S(L O)}=\frac{Z(U P)}{Z(L O)} \\
& \text { 2. ALPHA }=\frac{\operatorname{LOG}(W S(I P) / W S(L O))}{\operatorname{LOG}(Z(U P) / 2(L 0))}
\end{aligned}
$$


COUNT OF VIND SPEED VS. DIRECTION

EACH LINE OF CHARACTERS REPAESENTS 40 OBSERVATIONS

SPEED INCREASES WITH DISTANCE FROM CENTER OF CIRCLES

ASSOCIATED RANGES IN MPS ARE $0 \begin{array}{llllll}-4 & 4 & -10 & 10 & -20 & 20\end{array}$

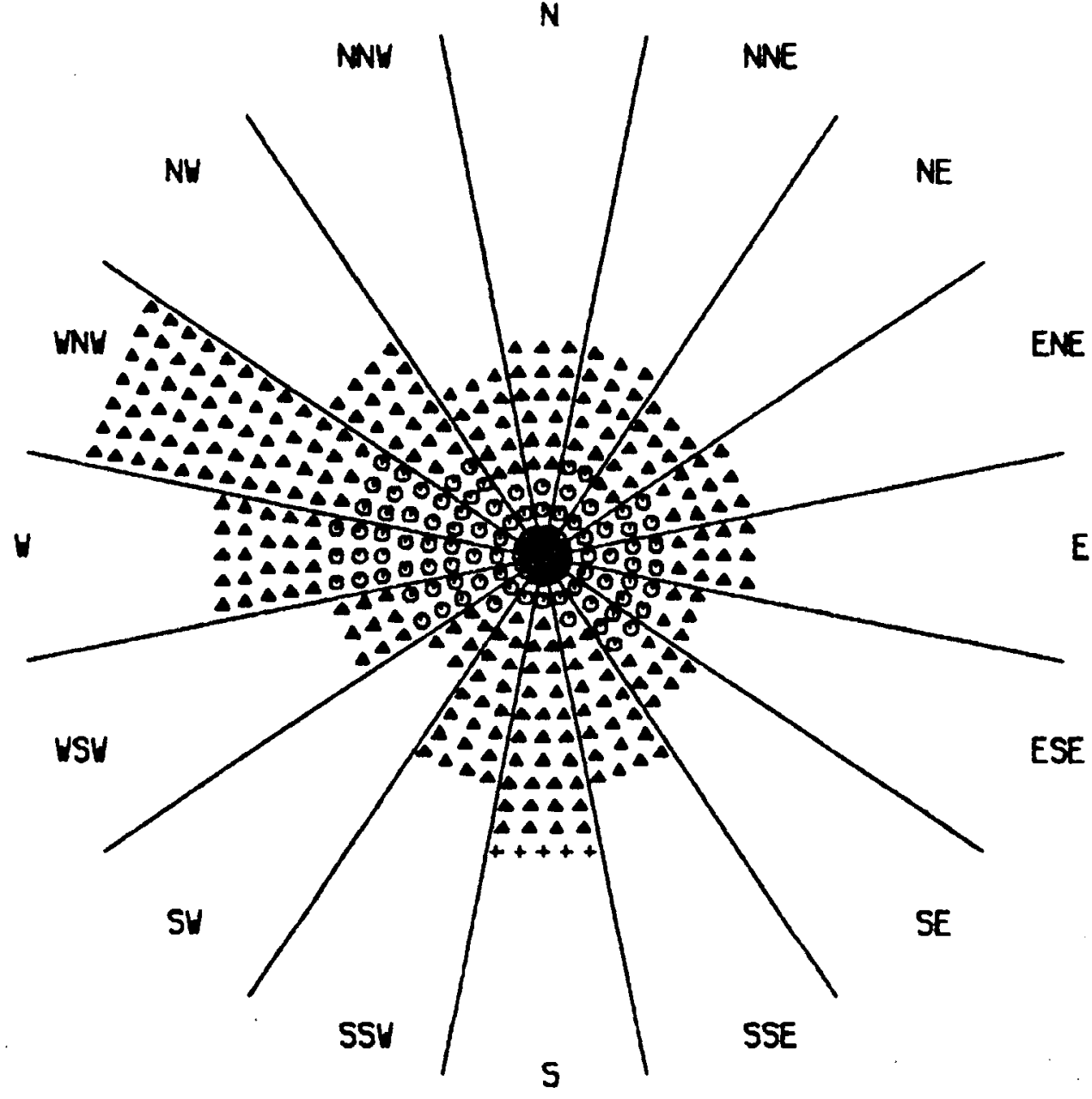

FIGURE 1. (NB) Wind Rose for Lowest Sensor Level 


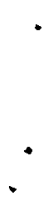


LUDINGTON, MICHIGAN 


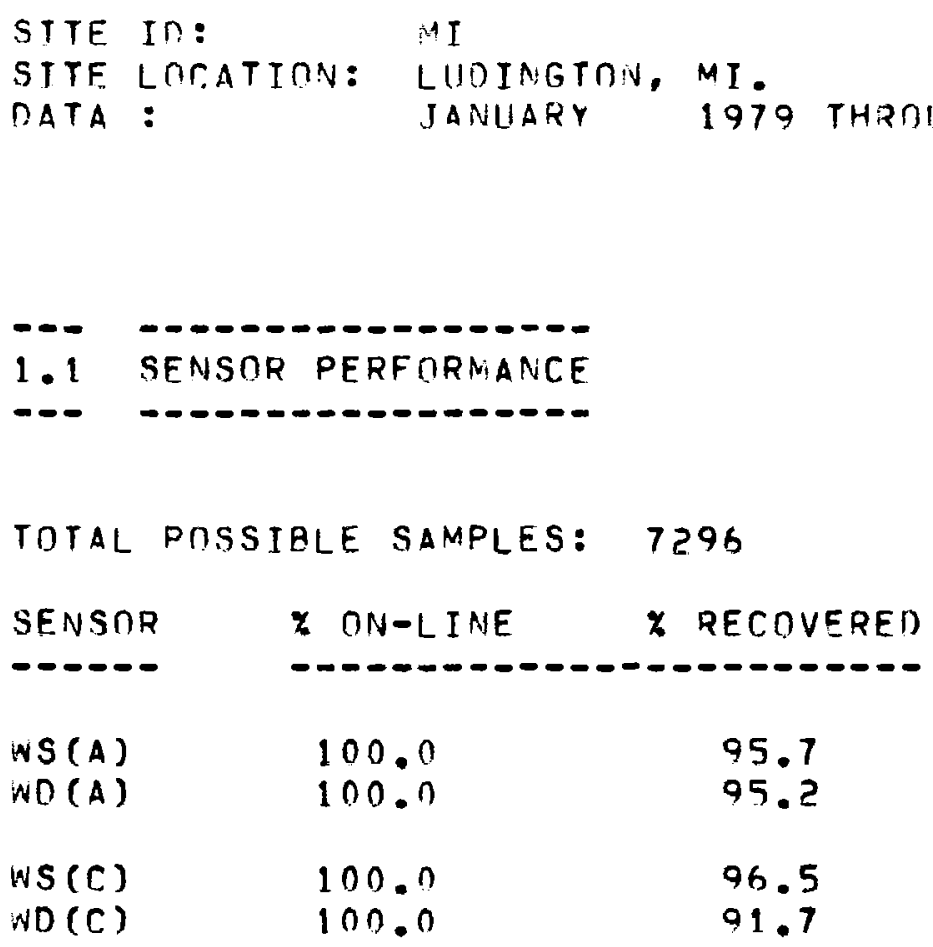

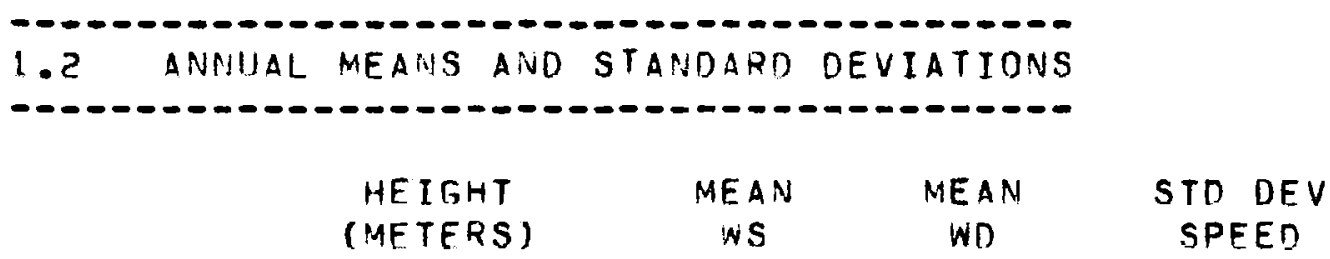

$\begin{array}{llllll}\text { SITE DATA } & \text { (A) } 45.7 & 7.2 & 2.08 .3 & 3.7 \\ & \text { (C) } 18.2 & 5.0 & 165.5 & 2.9\end{array}$

1.3 MAXIMUM WIND SPEED

-

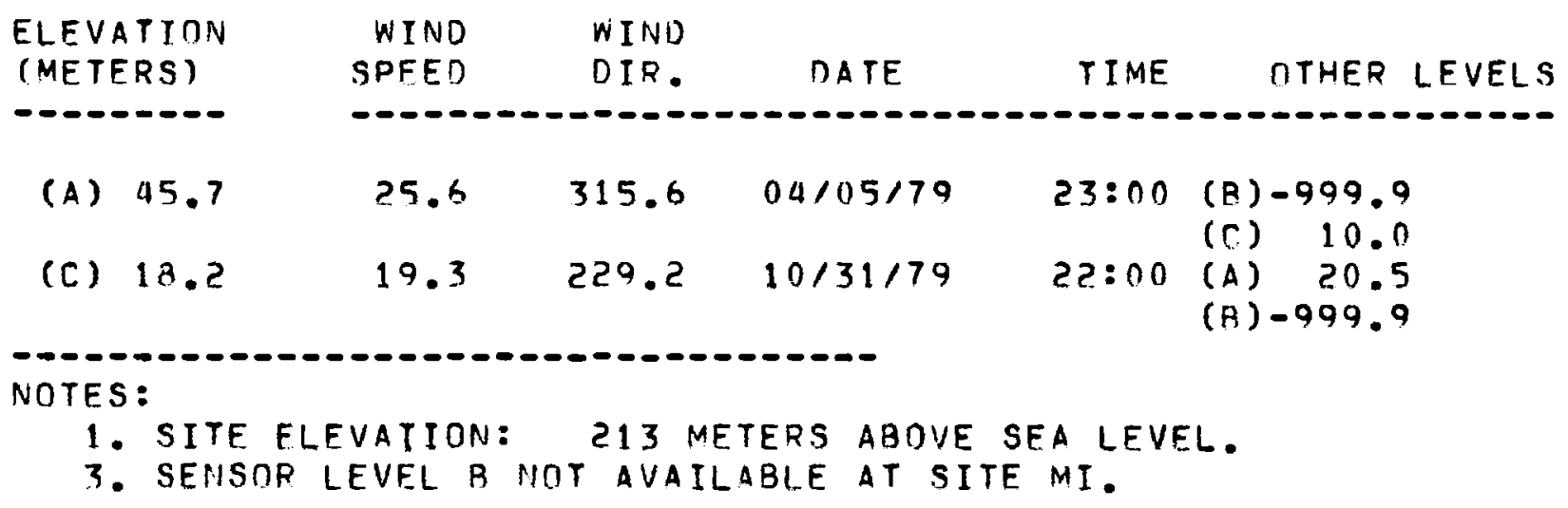


SITE ID: $\quad$ UI

SITE LOCATION: LUDINGTON, MI.

JANIJARY

1979 THROUGH OCTOBER

1979

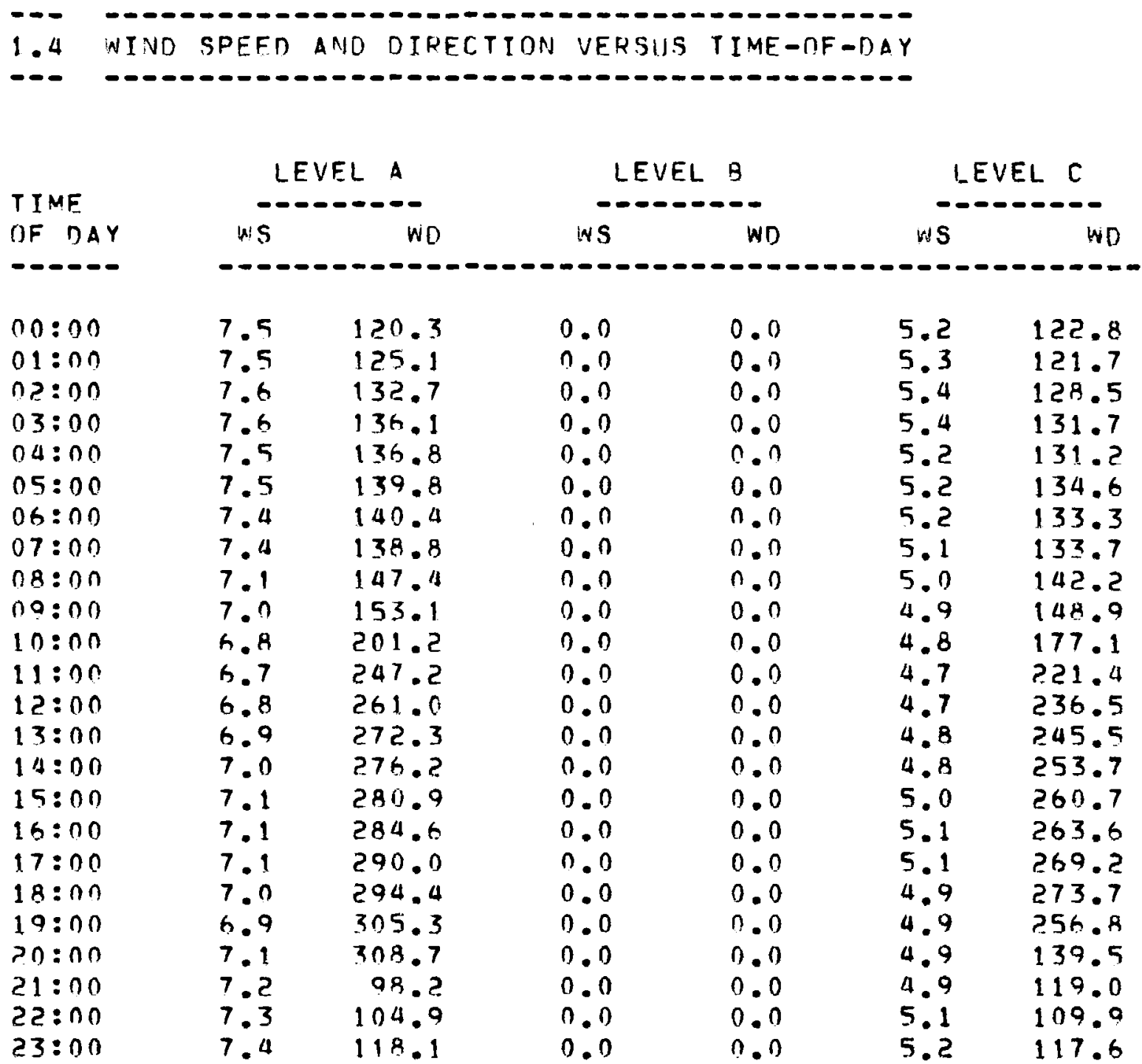


SITE IO:

SITF LOCATION:

DATA:
$4 T$

LUDINGTON, MI. JANUUARY

1979 THQOUGH OCTIBER

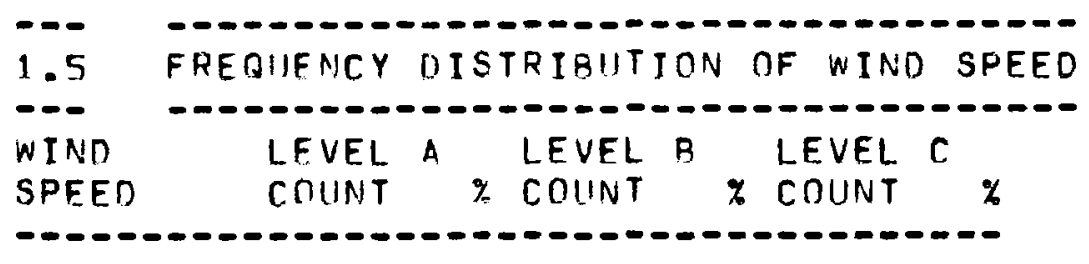

$\begin{array}{rrrrrrr}0.0-0.5 & 0 & 0.0 & 0 & 0.0 & 72 & 1.0 \\ 0.5-1.0 & 48 & 0.7 & 0 & 0.0 & 278 & 3.9 \\ 1.0-1.5 & 105 & 1.5 & 0 & 0.0 & 335 & 4.8 \\ 1.5-2.0 & 188 & 2.7 & 0 & 0.0 & 367 & 5.2 \\ 2.0-2.5 & 256 & 3.7 & 0 & 0.0 & 390 & 5.5 \\ 2.5-3.0 & 280 & 4.0 & 0 & 0.0 & 427 & 6.1 \\ 3.0-3.5 & 282 & 4.0 & 0 & 0.0 & 475 & 6.7 \\ 3.5-4.0 & 319 & 4.6 & 0 & 0.0 & 471 & 6.7 \\ 1.0-4.5 & 322 & 4.6 & 0 & 0.0 & 493 & 7.0 \\ 4.5-5.0 & 348 & 5.0 & 0 & 0.0 & 476 & 6.8 \\ 5.0-5.5 & 348 & 5.0 & 0 & 0.0 & 501 & 7.1 \\ 5.5-6.0 & 326 & 4.7 & 0 & 0.0 & 475 & 6.7 \\ 6.0-6.5 & 382 & 5.5 & 0 & 0.0 & 393 & 5.6 \\ 6.5-7.0 & 388 & 5.6 & 0 & 0.0 & 352 & 5.0 \\ 7.0-7.5 & 384 & 5.5 & 0 & 0.0 & 280 & 4.0 \\ 7.5-8.0 & 354 & 5.1 & 0 & 0.0 & 223 & 3.2 \\ 8.0-8.5 & 398 & 5.7 & 0 & 0.0 & 198 & 2.8 \\ 8.5-9.0 & 300 & 4.3 & 0 & 0.0 & 161 & 2.3 \\ 9.0-9.5 & 257 & 3.7 & 0 & 0.0 & 141 & 2.0 \\ 9.5-10.0 & 248 & 3.6 & 0 & 0.0 & 104 & 1.5 \\ 10.0-11.0 & 432 & 6.2 & 0 & 0.0 & 158 & 2.2 \\ 11.0-12.0 & 308 & 4.4 & 0 & 0.0 & 121 & 1.7 \\ 12.0-13.0 & 244 & 3.5 & 0 & 0.0 & 66 & 0.9 \\ 13.0-14.0 & 156 & 2.2 & 0 & 0.0 & 33 & 0.5 \\ 14.0-15.0 & 117 & 1.7 & 0 & 0.0 & 29 & 0.4 \\ 15.0-16.0 & 60 & 0.9 & 0 & 0.0 & 16 & 0.2 \\ 16.0-17.0 & 40 & 0.6 & 0 & 0.0 & 3 & 0.0 \\ 17.0-13.0 & 37 & 0.5 & 0 & 0.0 & 2 & 0.0 \\ 18.0-19.0 & 25 & 0.4 & 0 & 0.0 & 2 & 0.0 \\ 19.0-20.0 & 11 & 0.2 & 0 & 0.0 & 2 & 0.0 \\ 20.0-21.0 & 99 & 0.1 & 0 & 0.0 & 0 & 0.0 \\ >21.0 & 12 & 0.2 & 0 & 0.0 & 0 & 0.0\end{array}$

LEVEL A LEVEL B LEVFL C

$$
95.7 \quad 0.0 \quad 96.5
$$


SITE IT:

STTE LOCATTON: LHOINGTON, MI.

DATA : JANUARY 1979 THROIJGH OETOBER 1979

1.6 CIIMULATIUF FREQUENCY DISTRIAUTION OF WIND SPEFO

\begin{tabular}{|c|c|c|c|c|c|c|}
\hline & LFV & $A$ & LEV & B & LEV & $\mathrm{L}$ \\
\hline$---\infty$ & $---\infty$ & $x---\infty$ & $-\infty-\infty$ & $---\infty$ & & \\
\hline NIND & CFD & $C F D$ & $C F D$ & $C F D$ & CFO & CFD \\
\hline SPEEN & (ARS) & $(x)$ & (ABS) & $(\%)$ & $(A B S)$ & $(\%)$ \\
\hline---- & $-\cdots-$ & ----0 & $-\cdots$ & $--\infty-\infty$ & 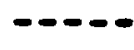 & $\ldots$ \\
\hline 0.5 & 0 & 0.00 & 0 & 0.00 & 72 & 1.02 \\
\hline 1.0 & 48 & 0.69 & 0 & 0.00 & 350 & 4.97 \\
\hline 1.5 & 153 & 2.19 & 0 & 0.00 & 685 & 9 \\
\hline 2. 0 & 341 & 4.88 & $n$ & 0.00 & 1052 & 14. \\
\hline 2.5 & 597 & 8.55 & 0 & 0.00 & 1442 & 20.47 \\
\hline 3.0 & 877 & 12.56 & 0 & 0.00 & 1869 & 26.5 \\
\hline 3.5 & 1159 & 16.60 & 0 & 0.00 & 2304 & 33.2 \\
\hline 4.0 & 1478 & 21.16 & 0 & 0.00 & 2815 & 39 \\
\hline 4.5 & 1800 & 25.77 & 0 & 0.00 & 3308 & $46 .{ }^{\circ}$ \\
\hline 5.0 & ?.148 & 30.76 & 0 & 0.00 & 3784 & 53.72 \\
\hline 5.5 & 2496 & 35.74 & 0 & 0.00 & 4285 & 83 \\
\hline 6.0 & 2822 & 40.41 & 0 & 0.00 & $476 n$ & 67.58 \\
\hline 6.5 & 3204 & 45.88 & 0 & 0.00 & 5153 & .15 \\
\hline 7.0 & $359 ?$ & 51.43 & $n$ & 0.00 & 5505 & 78 \\
\hline 7.5 & 3976 & 56.93 & 0 & 0.00 & 5785 & 82 \\
\hline 8.0 & 4330 & 62.00 & 0 & 0.00 & 6008 & 85 \\
\hline 8.5 & 4728 & 67.70 & 0 & 0.00 & 6206 & 88. \\
\hline 9.0 & 5028 & 71.99 & 0 & 0.00 & 6367 & 90 \\
\hline 9.5 & 5285 & 75.67 & 0 & 0.00 & 6508 & 92 \\
\hline 10.0 & 5533 & 79.22 & 0 & 0.00 & 6612 & 93.87 \\
\hline 11.0 & 5955 & 85.41 & 0 & 0.00 & 6770 & 96.11 \\
\hline 12.0 & 6273 & 89.82 & 0 & 0.00 & 6891 & 97. \\
\hline $13 . n$ & 6517 & 93.31 & 0 & 0.00 & 6957 & 98.76 \\
\hline 14.0 & 6673 & 95.55 & 0 & 0.00 & 6990 & 99.23 \\
\hline 15.0 & 6790 & 97.22 & 0 & 0.00 & 7019 & 99.65 \\
\hline 16.0 & 6850 & 98.08 & 0 & 0.00 & 7035 & 99.8 \\
\hline 17.0 & 6890 & 98.65 & 0 & 0.00 & 7038 & 99.9 \\
\hline 18.0 & 6027 & 99.13 & 0 & 0.00 & 7040 & 99. \\
\hline 19.0 & 6952 & 99.51 & 0 & 0.00 & 7042 & 99.5 \\
\hline ?. 0.0 & 6963 & 99.70 & 0 & 0.00 & 7044 & 100.00 \\
\hline 21.0 & $697 ?$ & 99.83 & 0 & 0.00 & 7044 & 100.0 \\
\hline 21.0 & 6984 & 100.00 & 0 & 0.00 & 7044 & 100 \\
\hline
\end{tabular}


STTE ID:

SITE LOCATTON: LUOINGTON, MI.

DATA : JANUARY 1979 THROUGH OCTOBER 1979

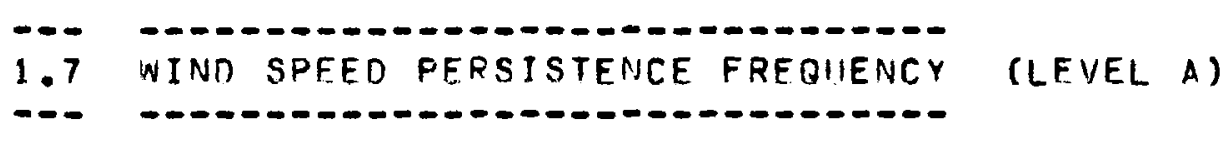

\begin{tabular}{|c|c|c|c|c|}
\hline HOURS & $\begin{array}{l}\text { PFRS } \\
<4\end{array}$ & $\begin{array}{l}\text { NIND } \\
4=12\end{array}$ & $\begin{array}{r}\text { SPEED, } \\
12-20\end{array}$ & $\begin{array}{l}M / S \\
\quad>20\end{array}$ \\
\hline--- & $\cdots-\infty$ & $=-0$ & $-\infty$ & $---\infty$ \\
\hline 1 & 98 & 78 & 38 & 2 \\
\hline 2 & 42 & 48 & 21 & 1 \\
\hline 3 & 31 & 38 & 15 & 0 \\
\hline 4 & 19 & 22 & 8 & 1 \\
\hline 5 & 15 & 26 & 8 & 0 \\
\hline 6 & 24 & 15 & 7 & ? \\
\hline 7 & 9 & 13 & 7 & 0 \\
\hline 8 & 11 & 14 & 7 & 0 \\
\hline 9 & 6 & $1 n$ & 6 & 0 \\
\hline 10 & 4 & 13 & 2 & 0 \\
\hline 11 & 8 & 11 & 3 & 1 \\
\hline $1 ?$ & 8 & 9 & 1 & 0 \\
\hline 13 & 3 & 12 & 2 & 0 \\
\hline 14 & 8 & 9 & 1 & 0 \\
\hline 15 & 4 & 7 & 1 & 0 \\
\hline 16 & 3 & 10 & 1 & 0 \\
\hline 17 & 2 & 9 & 1 & 0 \\
\hline 18 & 1 & 5 & 0 & 0 \\
\hline 19 & 1 & 5 & $n$ & 0 \\
\hline 20 & 3 & 8 & 0 & 0 \\
\hline 21 & 0 & 8 & 0 & $n$ \\
\hline 22 & 1 & 3 & 2 & 0 \\
\hline 23 & 0 & 3 & 1 & 0 \\
\hline$>23$ & 3 & 54 & 2 & \\
\hline
\end{tabular}




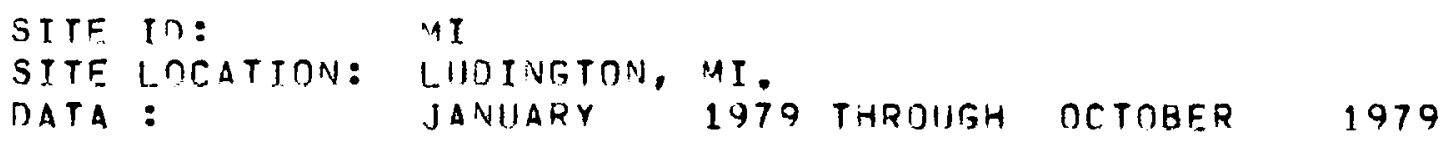

NNE

$$
0.00 \quad 0.00 \quad 0.140 .00
$$

0.00

$0.14 \quad 0.00$

10.07

0.00

8.90

NE.

ENE

0.00

0.00

$0.2^{n}$

0.00

7.46

0.00

6.81

0.00

n. ni)

0.74

0.00

5.00

0.00

4.49

$E$

ESE

$\begin{array}{ll}0.00 & 0.00 \\ 0.00 & 0.00\end{array}$

0.00

0.09

0.00

3.78

0.00

4.37

0.100 .00

3.89

0.00

4.61

$S E$

SSE

0.00

0.00

0.260 .00

5.53

0.00

6.52

$0.00 \quad 0.00$

0.35

0.00

6.87

0.00

6.29

SSW

SW

0.00

0.00

0.440 .00

5.03

0.00

5.12

$0.00 \quad 0.00$

0.310 .00

6.11

0.00

7.51

0.00

0.00

0.3

13.60

$0.0 n$

14.59

0.00

0.00

0.00

8.22

0.00

5.49

0.00

0.00

3.94

0.00

3.62

0.00

0.00

0.00

0.00

0.770 .00

2.91

0.00

2.67

NW

NNW

0.00

0.770 .00

4.60

5.48

5.87

0.00

4.07

$0.43 \quad 0.00$

0.00

4.83

4.90

NDTES:

$$
\text { 1. } \frac{\text { WS (IIP) }}{N S(L O)}=\frac{Z(U P)^{A L P H A}}{Z(L O)}
$$

$$
\text { 2. ALPHA }=\frac{\operatorname{LOG}(\text { WS }(U P) / N S(L O))}{\operatorname{LOG}(Z(U P) / 7(\operatorname{LO}))}
$$




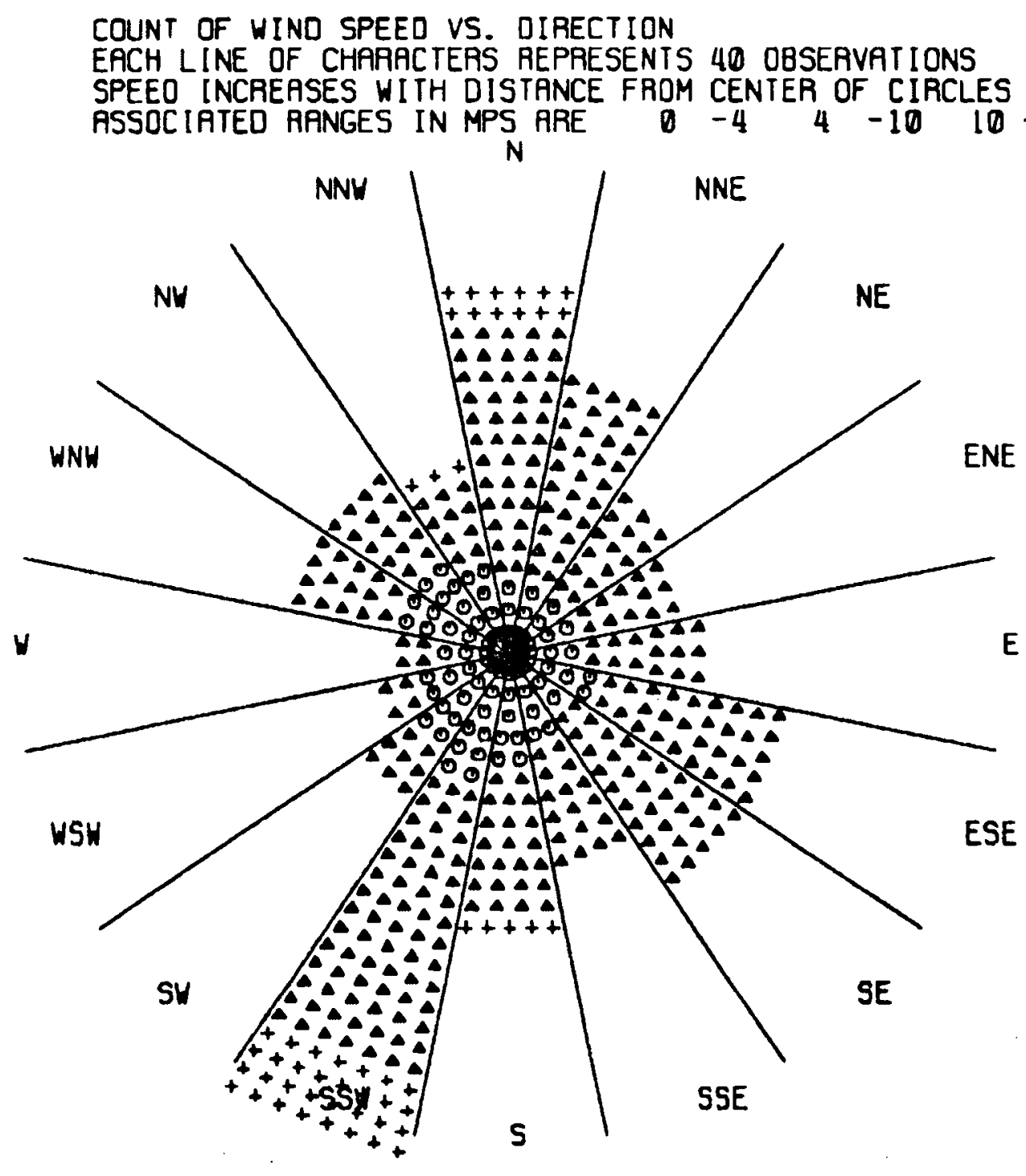

FIGURE 1. (MI) Wind Rose for Lowest Sensor Level 
.

- 
MONTAUK POINT, NEW YORK 
SITE IO: NY

SITE LOCATION: MONTAUK POINT, NY.

DATA : JANIIARY 1979 THRDUGH DECEMBER 1979

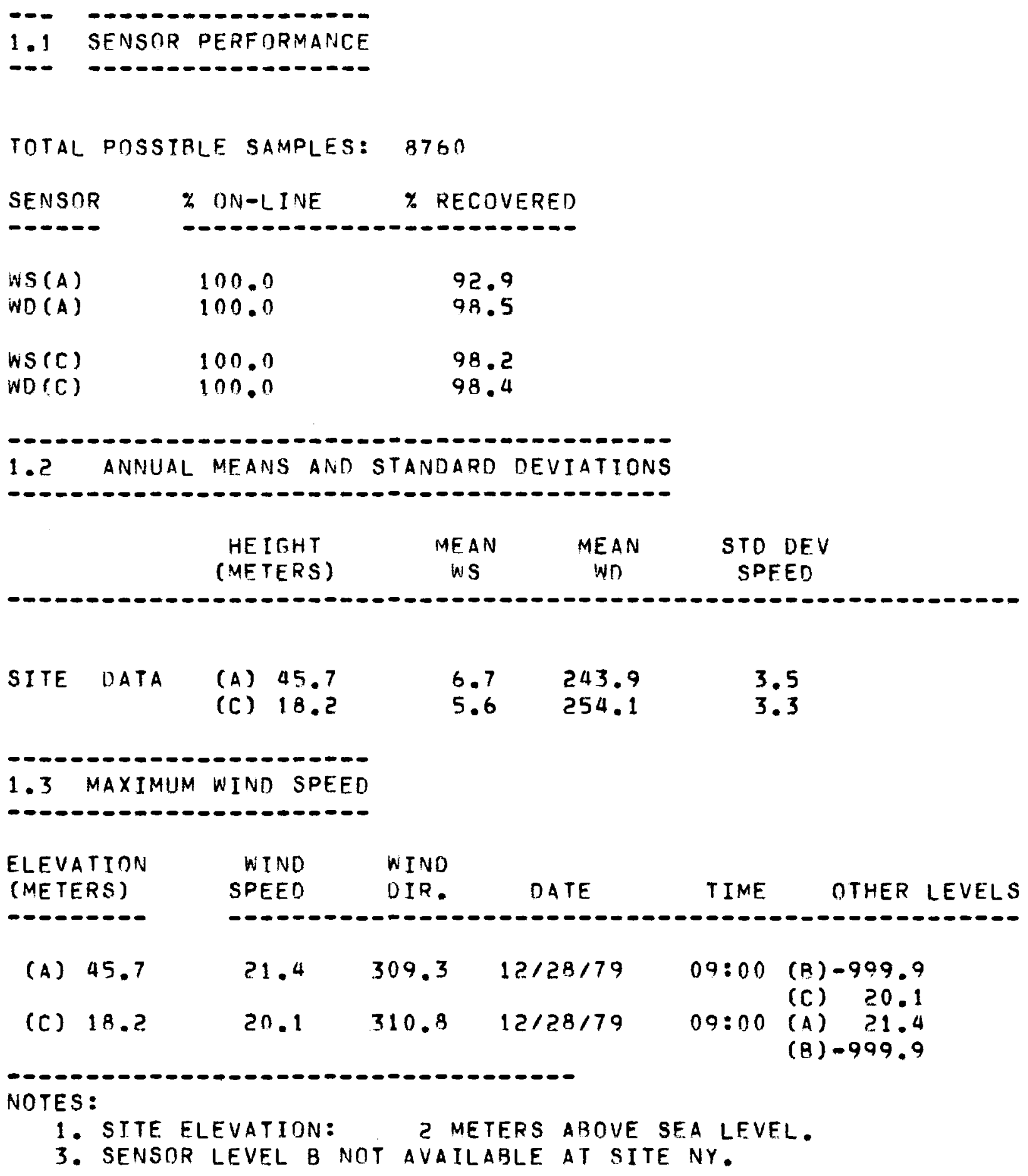


$\begin{array}{llll}\text { STTE ID: } & \text { NY } & & \\ \text { SITE LDCATTON: } & \text { MONTAUK POINT, NY. } & \\ \text { DATA : } & \text { JANUARY } & 1979 \text { THROUGH DECEMBER } & 1979\end{array}$

1.4 WIND SPEED AND UIRECTION VERSIJS TIME-OF-DAY

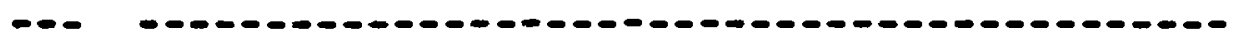

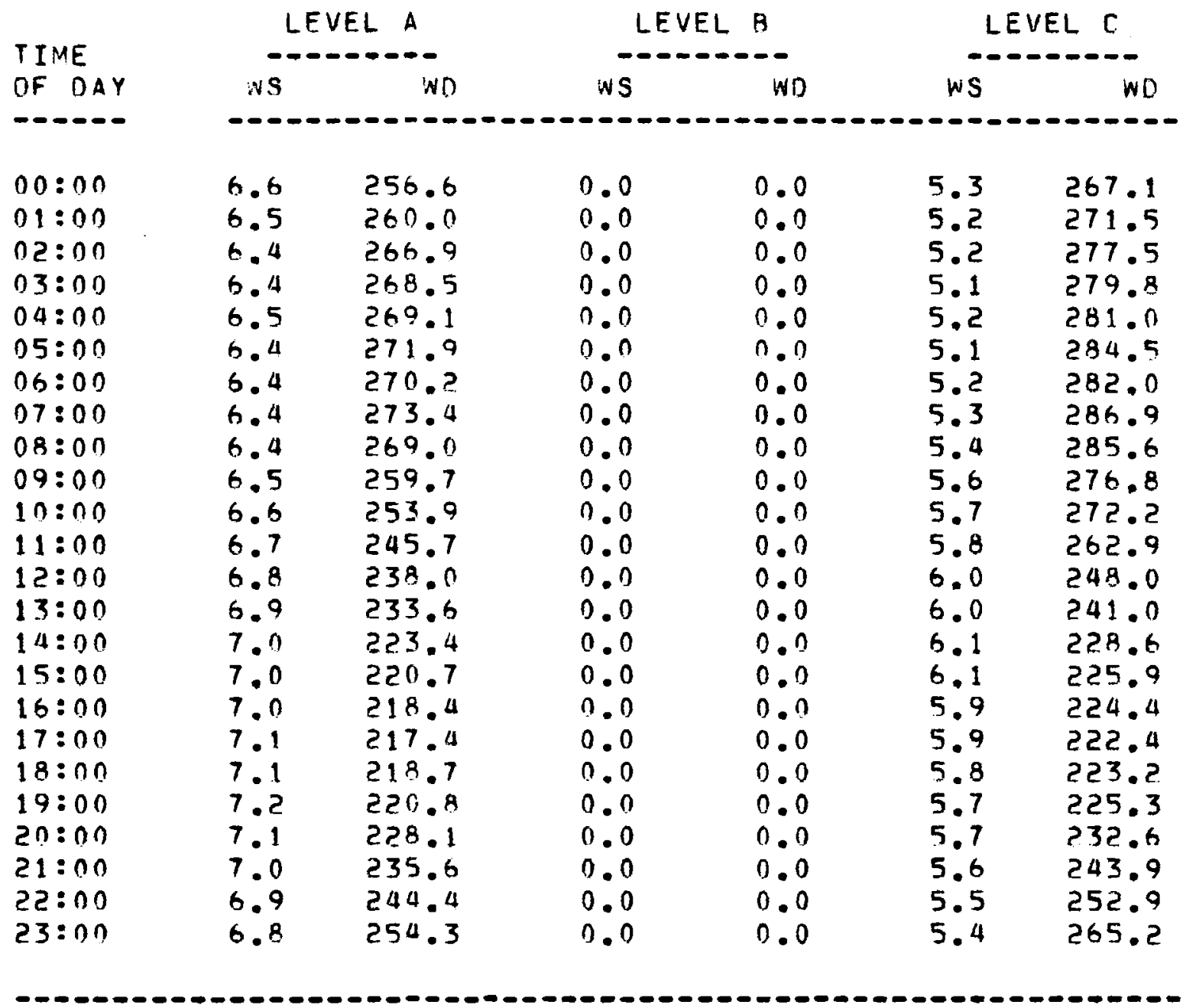


$\begin{array}{ll}\text { SITE ID: } & \text { NY } \\ \text { SITE LOCATION: MONTAUK POINT, NY. }\end{array}$

OATA : JANUARY 1979 THROUGH DECEMRER 1979

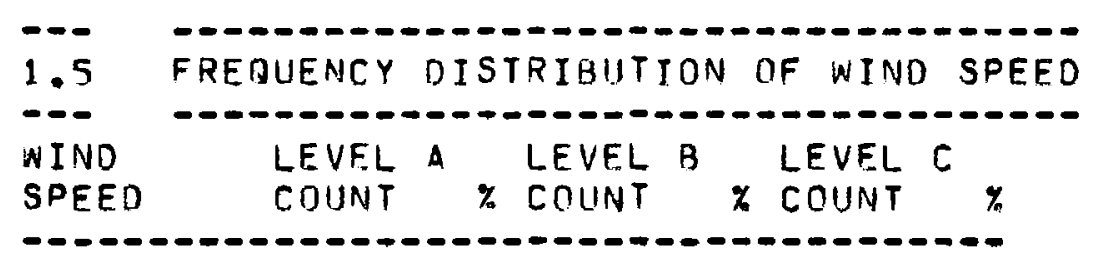

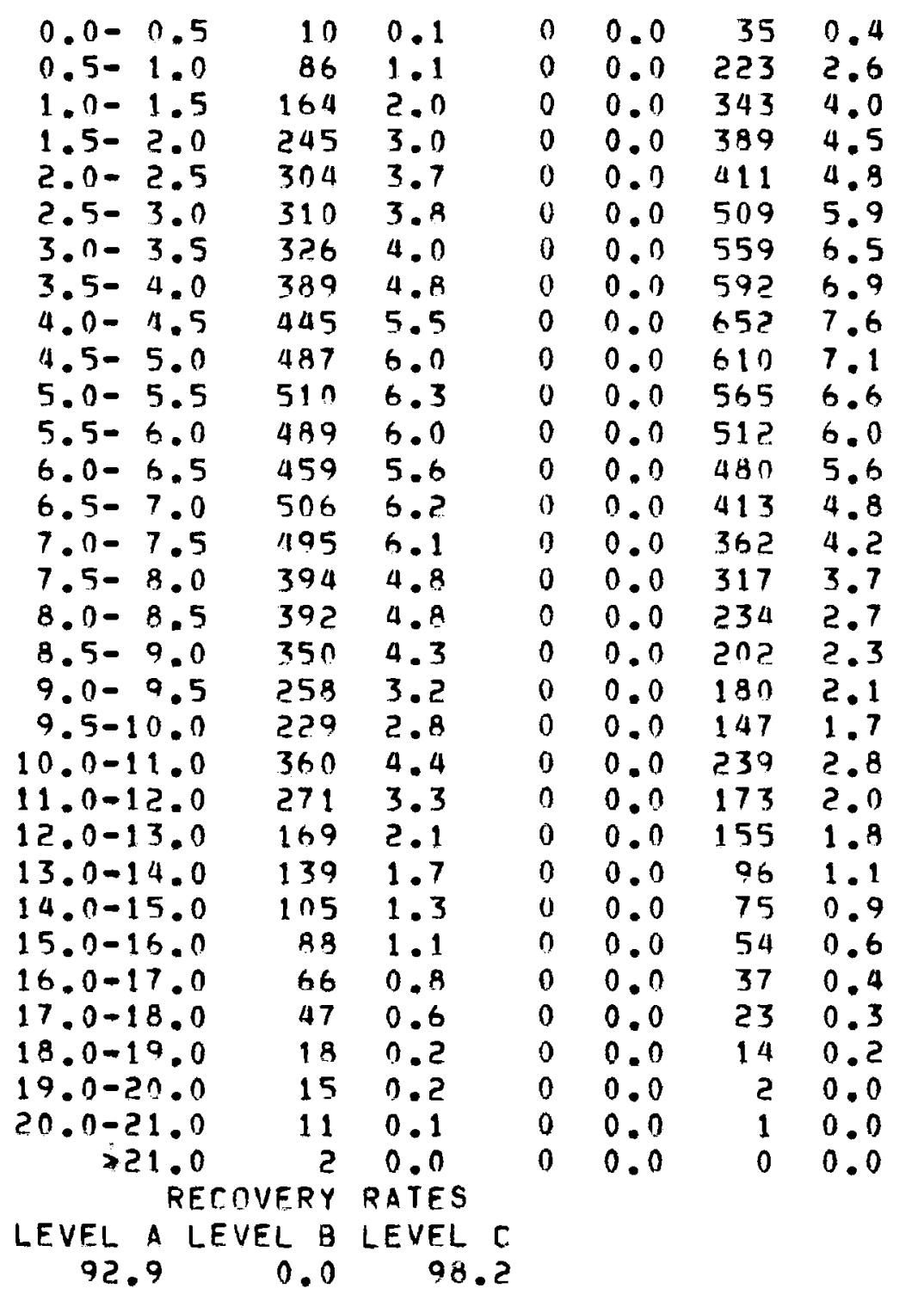


SITE ID:

NY

SITE LICATION: MONTAIIK POINT, NY.

JANUARY

1979 THROUGH DECEMRER

1979

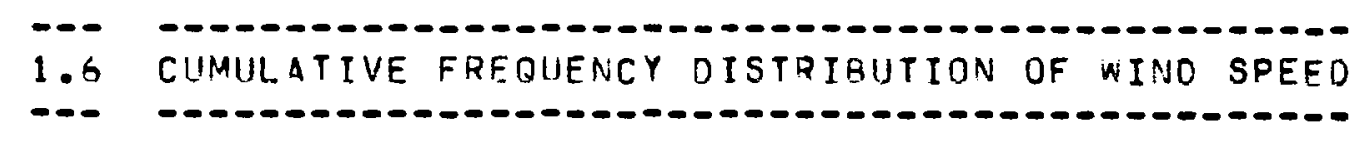

LEVEL A

---

WIND

SPEED

- - - -

0.5

1.0

1.5

2. 0

2. 5

3.0

3.5

4.0

4.5

5.0

5.5

6.0

6.5

7.0

7.5

8.0

8.5

9.0

9.5

10.0

11.0

12.0

13.0

14.0

15.0

16.0

17.0

18.0

19.0

$20 . ?$

21.0

$>21.0$

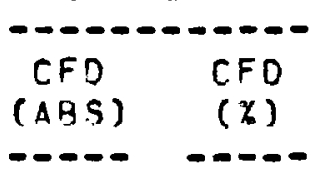

$10 \quad 0.12$

$96 \quad 1.18$

$260 \quad 3.19$

5056.20

$809 \quad 9.94$

$1119 \quad 13.75$

$1445 \quad 17.75$

183422.53

$2279 \quad 28.00$

$2766 \quad 33.98$

$3276 \quad 40.25$

$3765 \quad 46.26$

4224 51.90

$4730 \quad 58.12$

522564.20

$5619 \quad 69.04$

$6011 \quad 73.85$

636178.15

$6619 \quad 81.32$

$6848 \quad 84.14$

$7209 \quad 88.56$

$7479 \quad 91.89$

$7648 \quad 93.97$

$7787 \quad 95.68$

$7892 \quad 96.97$

$7980 \quad 98.05$

$8046 \quad 98.86$

$8093 \quad 99.43$

811199.66

$8126 \quad 99.84$

813799.98

8139100.00
LEVEL B

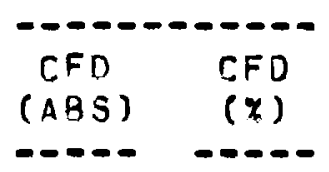

0.00

0.00

0.00

0.00

0.00

0.00

0.00

0.00

0.00

0.00

0.00

0.00

0.00

0.00

0.00

0.00

0.00

0.00

0.00

0.00

0.00

0.00

0.00

0.00

0.00

0.00

0.00

0.00

0.00

0.00

0.00

0.00
LEVEL C

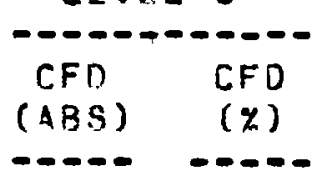

350.41

$258 \quad 3.00$

$601 \quad 6.99$

$990 \quad 11.51$

140116.28

$1910 \quad 22.20$

246928.70

306135.58

$3713 \quad 43.15$

432350.24

$4888 \quad 56.81$

$5400 \quad 62.76$

$5880 \quad 68.34$

629373.14

$6655 \quad 77.35$

$6972 \quad 81.03$

$7206 \quad 83.75$

$7408 \quad 86.10$

$7588 \quad 88.19$

$7735 \quad 89.90$

$7974 \quad 0.63$

814794.69

830296.49

$8398 \quad 97.61$

$8473 \quad 98.48$

$8527 \quad 99.11$

856499.54

858799.80

860199.97

$9603 \quad 99.90$

8604100.00

8604100.00 
$\begin{array}{llll}\text { SITE ID: } & \text { NY } & & \\ \text { SITE LOCATION: } & \text { MONTAUK POINT, NY. } & \\ \text { DATA : } & \text { JANUARY } & 1979 \text { THROUGH OECEMBER } & 1979\end{array}$

1.7 WIND SPEED PERSISTENCE FREAUENCY (LEVEL A)

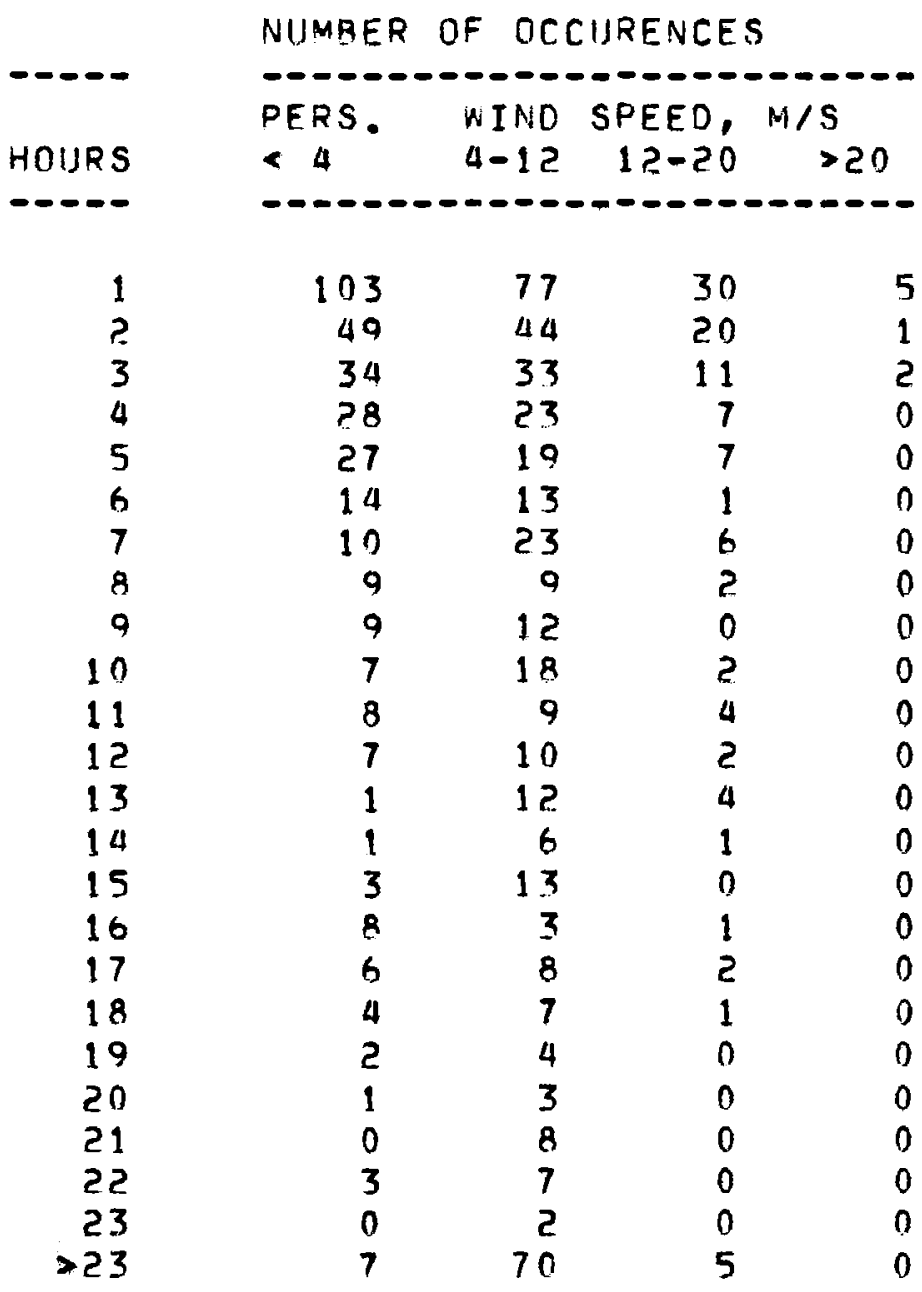


$\begin{array}{llll}\text { SITE ID: } & \text { NY } & & \\ \text { SITE LOCATION: } & \text { MONTAUK POINT, NY. } & \\ \text { DATA : } & \text { JAMUIHAY } & 1979 \text { THROUGH DECFMAER } & 1979\end{array}$

1.8 POWER LAW EXPONENT AND W/D DISTRIEUTION

WIND DIR

(ELEV A) ALPHA ALPHA ALPHA ALPHA

$(A, B)(B, C)(A, C)(A, B, C) \quad \% A \quad \% B \quad \% C$

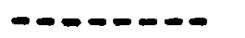

$(A, B)(B, C)(A, C)(A, B, C), \ldots$

$\begin{array}{lrllllll}\text { N } & 0.00 & 0.00 & 0.09 & 0.00 & 2.69 & 0.00 & 2.86 \\ \text { NNE } & 0.00 & 0.00 & 0.21 & 0.00 & 3.58 & 0.00 & 3.14 \\ \text { NE } & 0.00 & 0.00 & 0.19 & 0.00 & 5.46 & 0.00 & 5.25 \\ \text { ENE } & 0.00 & 0.00 & 0.30 & 0.00 & 3.75 & 0.00 & 3.58 \\ \text { E } & 0.00 & 0.00 & 0.13 & 0.00 & 2.86 & 0.00 & 3.05 \\ \text { ESE } & 0.00 & 0.00 & 0.38 & 0.00 & 2.38 & 0.00 & 2.21 \\ \text { SE } & 0.00 & 0.00 & 0.39 & 0.00 & 4.45 & 0.00 & 4.77 \\ \text { SSE } & 0.00 & 0.00 & 0.34 & 0.00 & 6.11 & 0.00 & 5.97 \\ \text { S } & 0.00 & 0.00 & 0.33 & 0.00 & 7.02 & 0.00 & 7.73 \\ \text { SSW } & 0.00 & 0.00 & 0.31 & 0.00 & 8.83 & 0.00 & 9.38 \\ \text { SW } & 0.00 & 0.00 & 0.23 & 0.00 & 9.21 & 0.00 & 9.00 \\ \text { WSW } & 0.00 & 0.00 & 0.24 & 0.00 & 7.59 & 0.00 & 7.50 \\ \text { W } & 0.00 & 0.00 & 0.15 & 0.00 & 10.36 & 0.00 & 8.26 \\ \text { WNW } & 0.00 & 0.00 & 0.23 & 0.00 & 11.17 & 0.00 & 10.47 \\ \text { NW } & 0.00 & 0.00 & 0.01 & 0.00 & 9.58 & 0.0 n & 11.03 \\ \text { NNW } & 0.00 & 0.00 & 0.05 & 0.00 & 4.96 & 0.00 & 5.81\end{array}$

NOTES:

1. $\frac{W S(1 J P)}{W S(L O)}=\frac{Z(U P)^{A L P H A}}{Z(L O)}$

$\operatorname{Lng}($ WS (UP) / WS (L_0))

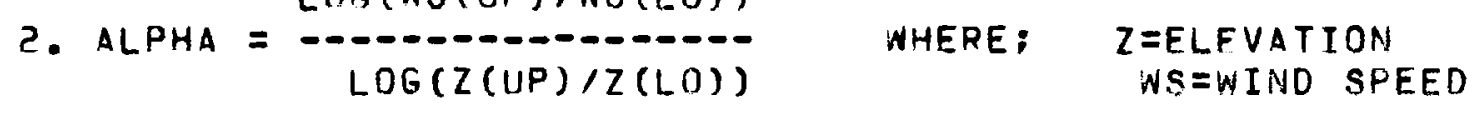




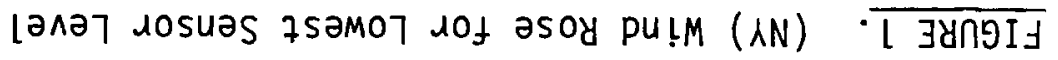

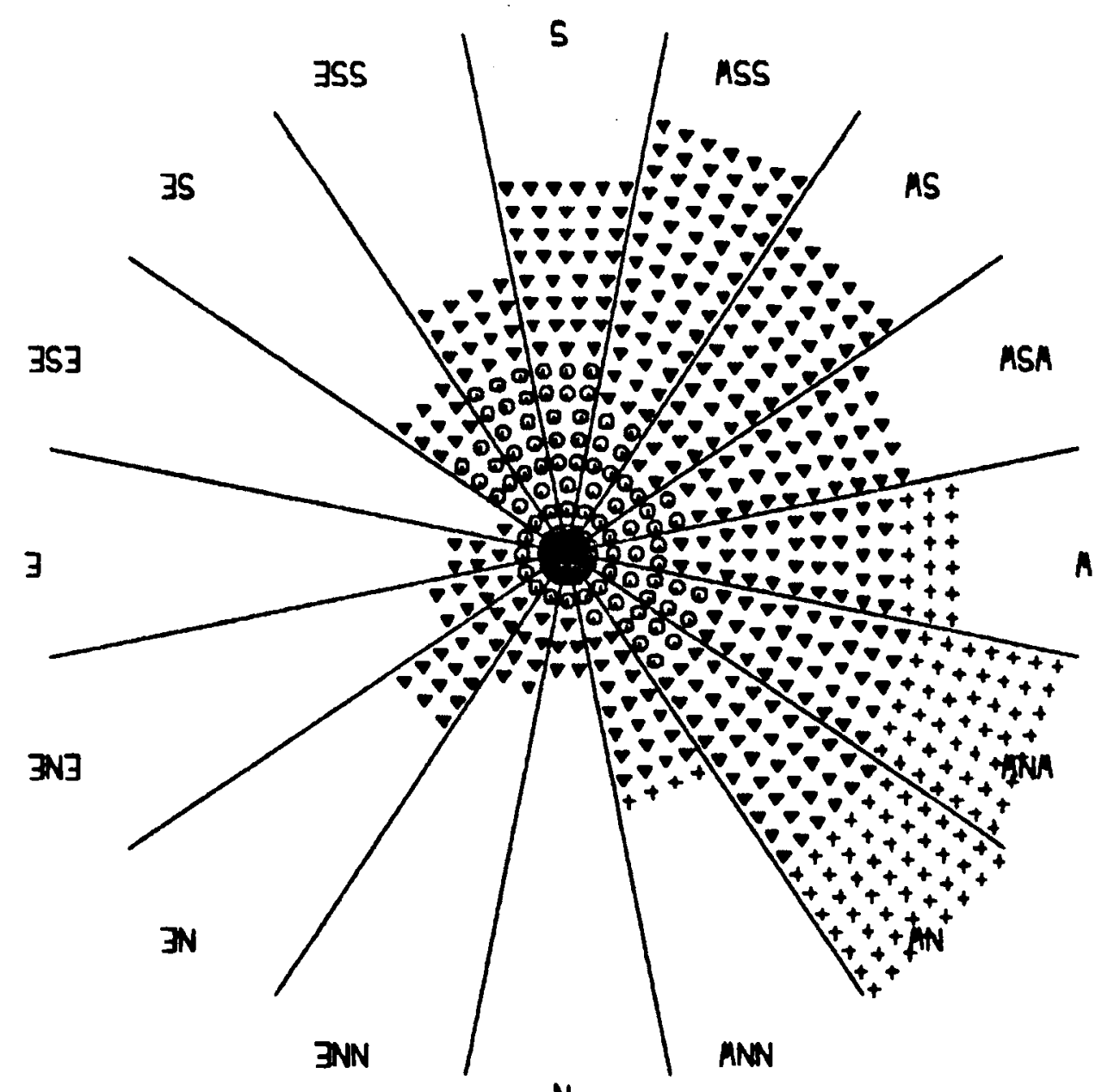

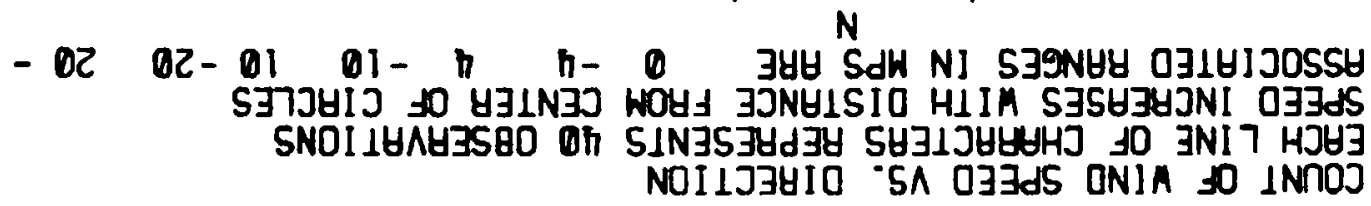




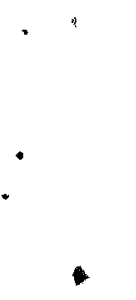

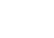


POINT ARENA, CALIFORNIA 
$\begin{array}{llll}\text { SITE IO: } & \text { CA } & \\ \text { SITE LOCATION: } & \text { POINT ARENA, CA. } & \\ \text { OATA : } & \text { JANUARY } & & \\ & & \end{array}$

1.1 SENSOR PERFURMANCE

TOTAL POSSIBLE. SAMPLES: 8760

$\begin{array}{lcc}\text { SENSOR } & \% \text { UN-LINE } & \text { \% RECOVERED } \\ \text { WS(A) } & 100.0 & 86.2 \\ \text { ND(A) } & 100.0 & 94.3 \\ \text { WS(C) } & 100.0 & 93.2 \\ \text { WD(C) } & 100.0 & 83.8\end{array}$

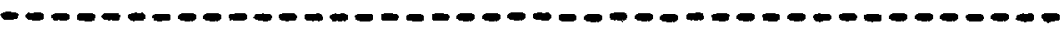

1.2 ANNUAL MEANS AND STANOARD DEVIATIONS

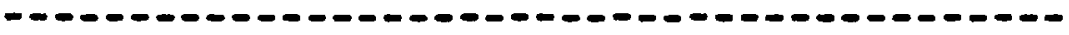

$\begin{array}{cccc}\text { HEIGHT } & \text { MEAN } & \text { MEAN } & \text { STD DEV } \\ \text { (METERS) } & \text { WS } & \text { WD } & \text { SPEED }\end{array}$

$\begin{array}{rrrrrr}\text { SITE DATA } & \text { (A) } & 45.7 & 6.4 & 5.7 & 3.4 \\ & \text { (C) } & 9.1 & 4.7 & 358.3 & 2.5\end{array}$

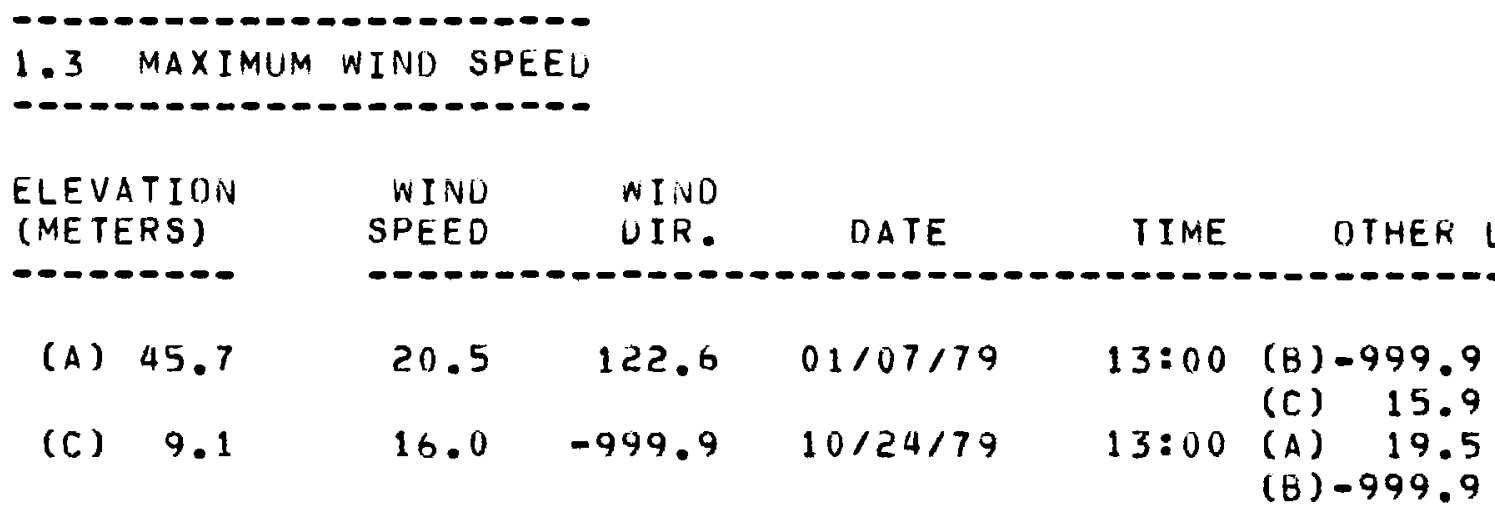

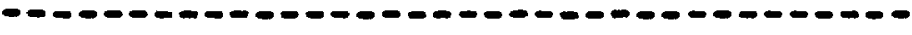

NOTES:

1. Site eleVATION: 21 meters above sea leVEl.

3. SENSOR LEVEL B NOT AVAILABLE AT SITE CA.

$$
C A-01
$$


SITE IU: CA

SITE LOCATION: POINI ARENA, CA.

UATA : JANUARY 1979 THRDUGH DECEMBEF 1979

\begin{tabular}{|c|c|c|c|c|c|c|}
\hline \multirow{3}{*}{$\begin{array}{l}\text { TIME } \\
\text { UF DAY }\end{array}$} & \multicolumn{2}{|c|}{ LEVEL A } & \multicolumn{2}{|c|}{ LEVEL $\quad$ B } & \multicolumn{2}{|c|}{ LEVEL C } \\
\hline & & $---\infty$ & & $-\infty$ & - & ---0 \\
\hline & NS & Wo & WS & ND & ws & WD \\
\hline$-----\infty$ & $-\infty$ & $-\infty-\infty$ & $-\infty$ & $-\infty$ & $-0-0$ & $\cdots-\infty$ \\
\hline $00: 00$ & 6.4 & 19.1 & 0.0 & 0.0 & 4.4 & 15.1 \\
\hline $01: 00$ & 6.3 & 19.5 & 0.0 & 0.0 & 4.4 & 16.9 \\
\hline $02: 00$ & 6.2 & 18.3 & 0.0 & 0.0 & 4.4 & 18.4 \\
\hline ט 3:00 & 6.2 & 22.8 & 0.0 & 0.0 & 4.4 & 20.7 \\
\hline $04: 00$ & 6.1 & 27.2 & 0.0 & 0.0 & 4.3 & 23.3 \\
\hline $05: 00$ & 6.1 & 30.0 & 0.0 & 0.0 & 4.3 & 24.0 \\
\hline U6:00 & h. $?$ & 29.1 & 0.0 & 0.0 & 4.2 & 20.0 \\
\hline $07: 00$ & 5.8 & 28.6 & 0.0 & 0.0 & 4.3 & 14.3 \\
\hline $08: 00$ & 6.0 & 33.1 & 0.0 & 0.0 & 4.4 & 359.9 \\
\hline $09: 00$ & 6.2 & 12.3 & 0.0 & 0.0 & 4.7 & 339.9 \\
\hline $10: 00$ & 6.5 & 333.8 & 0.0 & 0.0 & 5.0 & 326.6 \\
\hline $11: 00$ & 6.7 & 313.1 & 0.0 & 0.0 & 5.2 & 318.2 \\
\hline $12: 00$ & 7.0 & 316.1 & 0.0 & 0.0 & 5.4 & 319.3 \\
\hline $13: 00$ & 7.0 & 319.3 & 0.0 & 0.0 & 5.4 & 321.5 \\
\hline $14: 00$ & 6.9 & $3 ? 4.1$ & 0.0 & 0.0 & 5.3 & 322.3 \\
\hline $15: 00$ & 6.8 & 332.2 & 0.0 & 0.0 & 5.3 & 329.5 \\
\hline $16: 00$ & 6.9 & 341.7 & 0.0 & 0.0 & 5.2 & 337.5 \\
\hline $17: 00$ & 6.8 & 353.9 & 0.0 & 0.0 & 5.0 & 348.5 \\
\hline $18: 00$ & 6.7 & 1.3 & 0.0 & 0.0 & 4.8 & 357.1 \\
\hline $19: 00$ & 6.6 & 6.2 & 0.0 & 0.0 & 4.6 & 2.2 \\
\hline $20: 00$ & 6.5 & 9.7 & 0.0 & 0.0 & 4.5 & 4.9 \\
\hline $21: 00$ & 6.3 & 14.0 & 0.0 & 0.0 & 4.4 & 8.1 \\
\hline $22: 00$ & 6.3 & 15.7 & 0.0 & 0.0 & 4.3 & 11.7 \\
\hline $23: 00$ & 6.4 & 15.0 & 0.0 & 0.0 & 4.4 & 13.8 \\
\hline
\end{tabular}




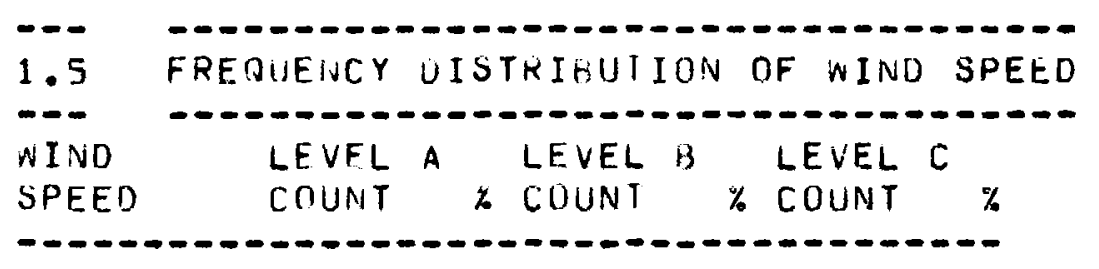

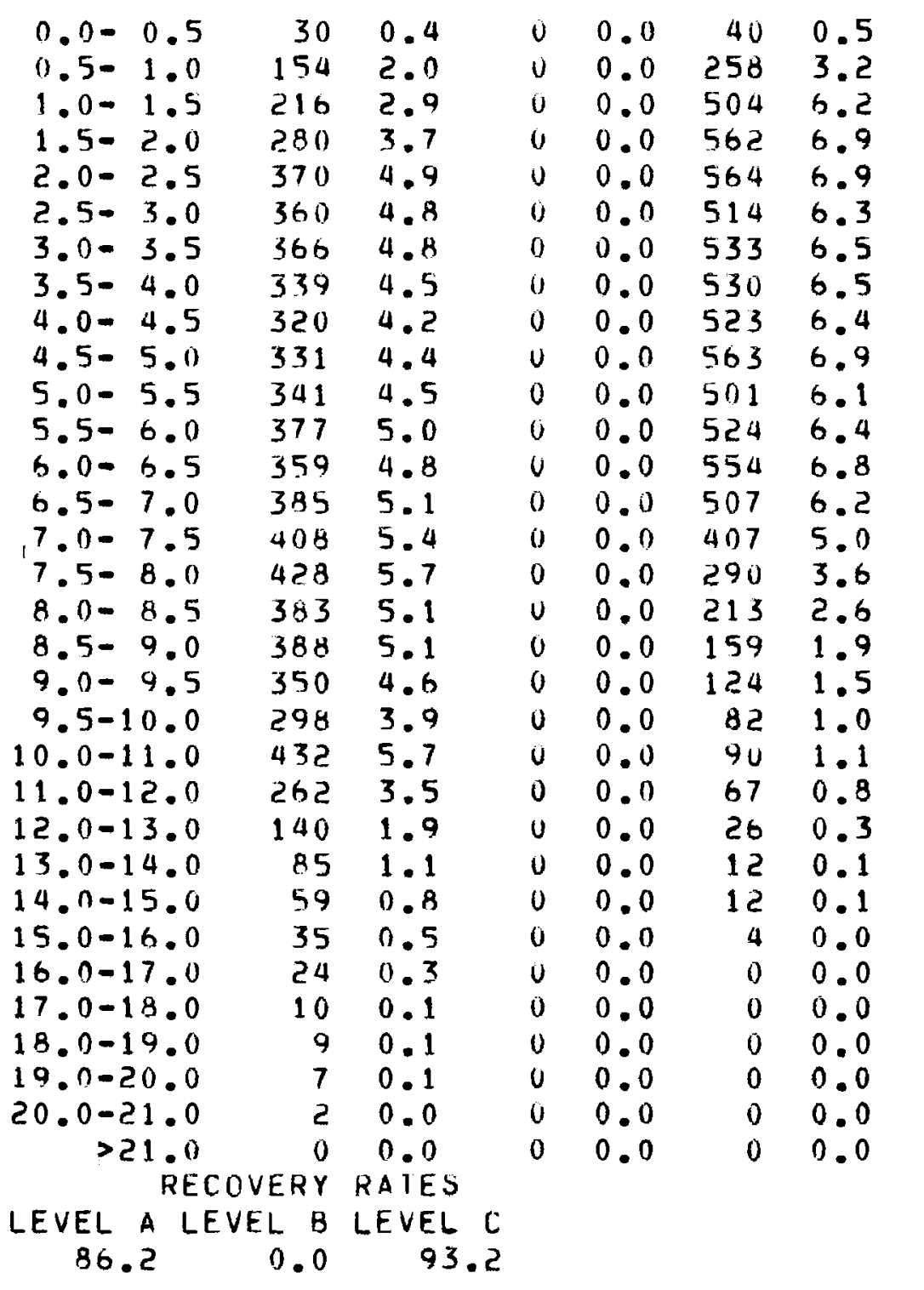


SITE ID: CA

SITE LOCATION: POINI ARENA, CA.

UATA : JANUARY 1974 THROUGH DECFMBEF 1979

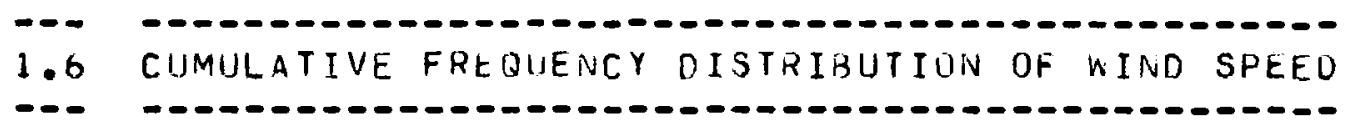

LEVEL A

WIND

SPEED

- - -

0.5

1.0

1.5

2. 0

2.5

3.0

3.5

4.0

4.5

5.0

5.5

6.0

6.5

7.0

7.5

8.0

8. 5

9.0

9.5

10.0

11.0

12.0

13.0

14.0

15.0

16.0

17.0

18.0

19.0

20.0

21.0

$>21.0$

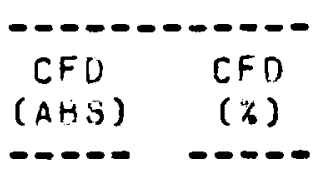

$\begin{array}{rr}30 & 0.40 \\ 184 & 2.44 \\ 400 & 5.30 \\ 680 & 9.01\end{array}$

$1050 \quad 13.91$

$1410 \quad 18.68$

$1776 \quad 23.55$

$2115 \quad 28.02$

243532.20

$2766 \quad 36.65$

$3107 \quad 41.16$

$3484 \quad 46.16$

$3843 \quad 50.91$

$4228 \quad 56.01$

$4636 \quad 61.42$

$5064 \quad 67.09$

$5447 \quad 72.10$

583577.31

018581.94

$6483 \quad 85.89$

$6915 \quad 91.61$

$7177 \quad 95.08$

$7317 \quad 96.94$

$7402 \quad 98.07$

$7461 \quad 98.85$

$7496 \quad 99.31$
$7520 \quad 99.63$

7520

7530

7539

99.63

99.76

99.68

99.97

7548100.00

$7548 \quad 100.00$
LEVEL $B$

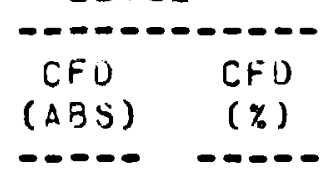

0

0

0

0

0

0

0

0

0

0

0

0

0

0

0

0

0

$0 \quad 0.00$

$0 \quad 0.00$

$0 \quad 0.00$

$0 \quad 0.00$

$0 \quad 0.00$

$0 \quad 0.00$

$0 \quad 0.00$

$0 \quad 0.00$

$0 \quad 0.00$

$0 \quad 0.00$

$0 \quad 0.00$

$0 \quad 0.00$

$0 \quad 0.00$

$0 \quad 0.00$

$0 \quad 0.00$
LEVEL C

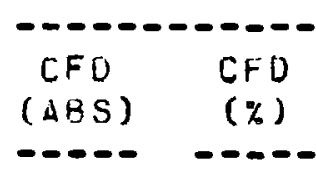

$\begin{array}{rr}40 & 0.49 \\ 298 & 3.65 \\ 802 & 9.82 \\ 1364 & 16.71\end{array}$

$1928 \quad 23.62$

$2442 \quad 29.92$

$2975 \quad 36.44$

$3505 \quad 42.94$

$4028 \quad 49.34$

459156.24

$5092 \quad 62.38$

$5616 \quad 68.80$

$6170 \quad 75.58$

$6677 \quad 81.80$

$7084 \quad 86.78$

7374
750.33

$7587 \quad 92.94$

$7746 \quad 94.89$

$7870 \quad 96.41$

795297.42

804298.52

810999.34

813599.66

$8147 \quad 99.80$

$8159 \quad 99.95$

8163100.00

8163100.00

8163100.00

8163100.00

8163100.00

8163100.00

8163100.00 
SITE ID:

SITE LOCATION: DATA :
$C A$

POINT ARENA, CA. JANUARY

1.7

$---$

WINO SPEED PERSTSTENCE FRENUENCY

(LEVEL A)

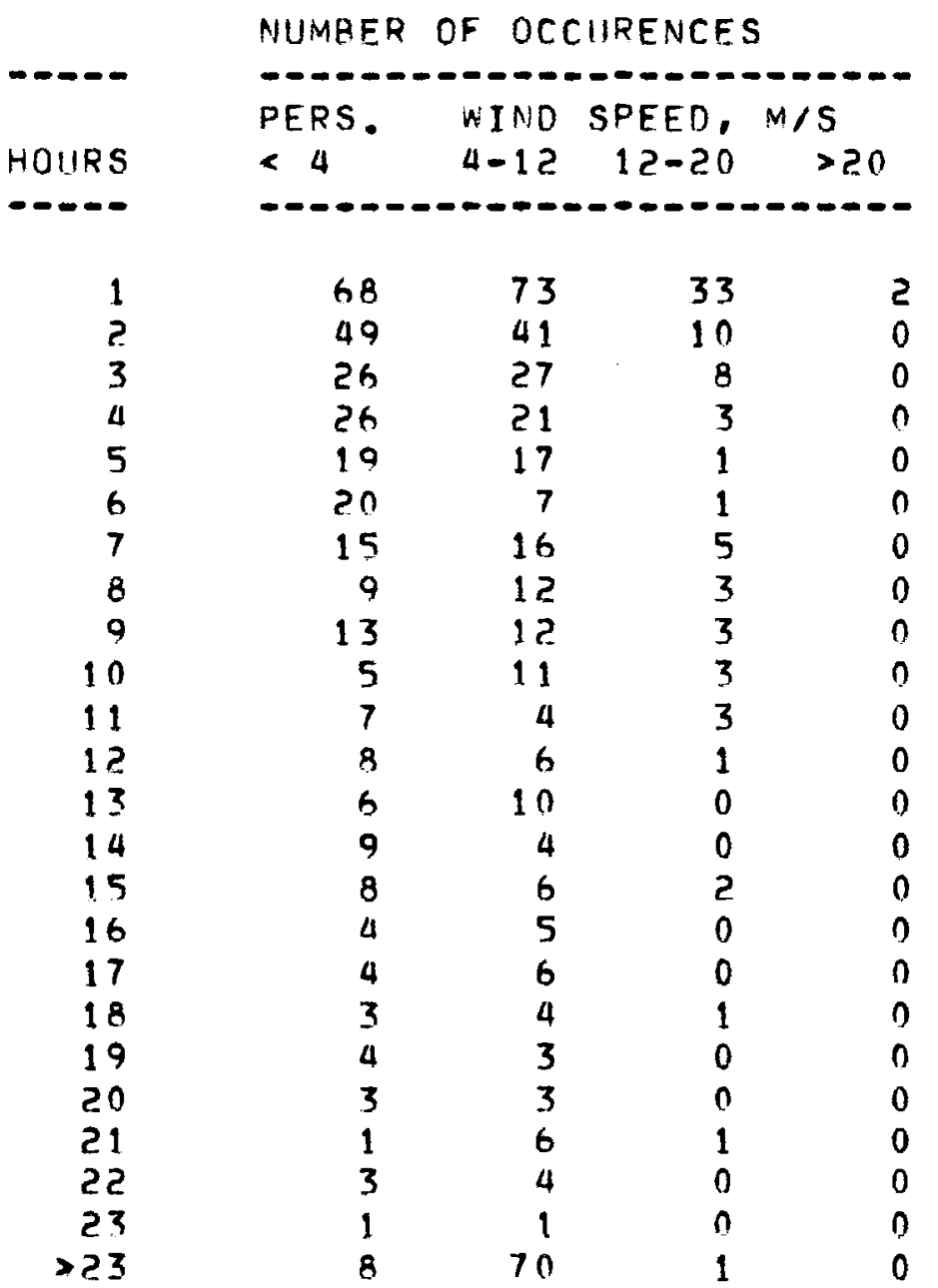


SITE ID: CA

STTE LICATION: POINT ARENA, CA.

DATA : JAMUARY 1979 THROUGH DECEMRER 1979

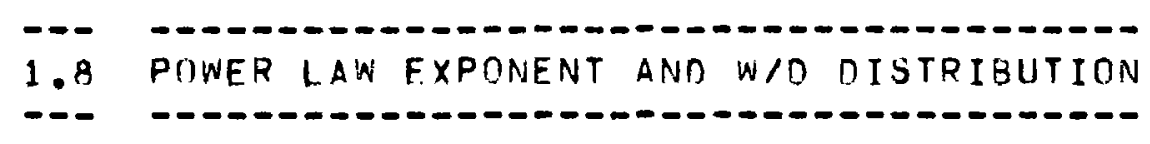

WIND DIR

(ELEV A)

ALPHA ALPHA ALPHA ALPHA

$(A, B)(B, C)(A, C)(A, B, C) \quad \% A \quad \times B \quad \forall C$

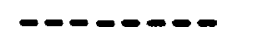

$\begin{array}{lrrrrrrr}\text { N } & 0.00 & 0.00 & 0.14 & 0.00 & 12.52 & 0.00 & 12.95 \\ \text { NNE } & 0.00 & 0.00 & 0.18 & 0.00 & 2.97 & 0.00 & 2.95 \\ \text { NE } & 0.00 & 0.00 & 0.22 & 0.00 & 1.17 & 0.00 & 1.75 \\ \text { ENE } & 0.00 & 0.00 & 0.24 & 0.00 & 0.89 & 0.00 & 1.89 \\ \text { E } & 0.00 & 0.00 & 0.45 & 0.00 & 1.51 & 0.00 & 1.31 \\ \text { ESE } & 0.00 & 0.00 & 0.54 & 0.00 & 3.72 & 0.00 & 1.78 \\ \text { SE } & 0.00 & 0.00 & 0.22 & 0.00 & 15.33 & 0.00 & 10.68 \\ \text { SSE } & 0.00 & 0.00 & 0.19 & 0.00 & 8.80 & 0.00 & 8.22 \\ \text { S } & 0.00 & 0.00 & 0.11 & 0.00 & 2.12 & 0.00 & 1.64 \\ \text { SSW } & 0.00 & 0.00 & 0.21 & 0.00 & 1.17 & 0.00 & 0.32 \\ \text { SW } & 0.00 & 0.00 & 0.08 & 0.00 & 0.90 & 0.00 & 0.78 \\ \text { WSN } & 0.00 & 0.00 & 0.12 & 0.00 & 0.93 & 0.00 & 0.60 \\ \text { W } & 0.00 & 0.00 & 0.24 & 0.00 & 1.23 & 0.00 & 1.15 \\ \text { NNW } & 0.00 & 0.00 & 0.29 & 0.00 & 1.96 & 0.00 & 1.58 \\ \text { NW } & 0.00 & 0.00 & 0.21 & 0.00 & 6.97 & 0.00 & 4.31 \\ \text { NNW } & 0.00 & 0.00 & 0.15 & 0.00 & 37.64 & 0.00 & 34.15\end{array}$

NOTES:

$$
\text { 1. } \frac{\text { WS (UP) }}{W S(L O)}=\frac{Z(U P)}{Z(L O)}
$$

ALPHA

2. $A L P H A=\frac{\operatorname{LOG}(W S(U P) / W S(L O))}{\operatorname{LOG}(Z(U P) / Z(L O))}$

WHERE: $\quad Z=E L E V A T T O N$

WS $=W I N D$ SPEED 


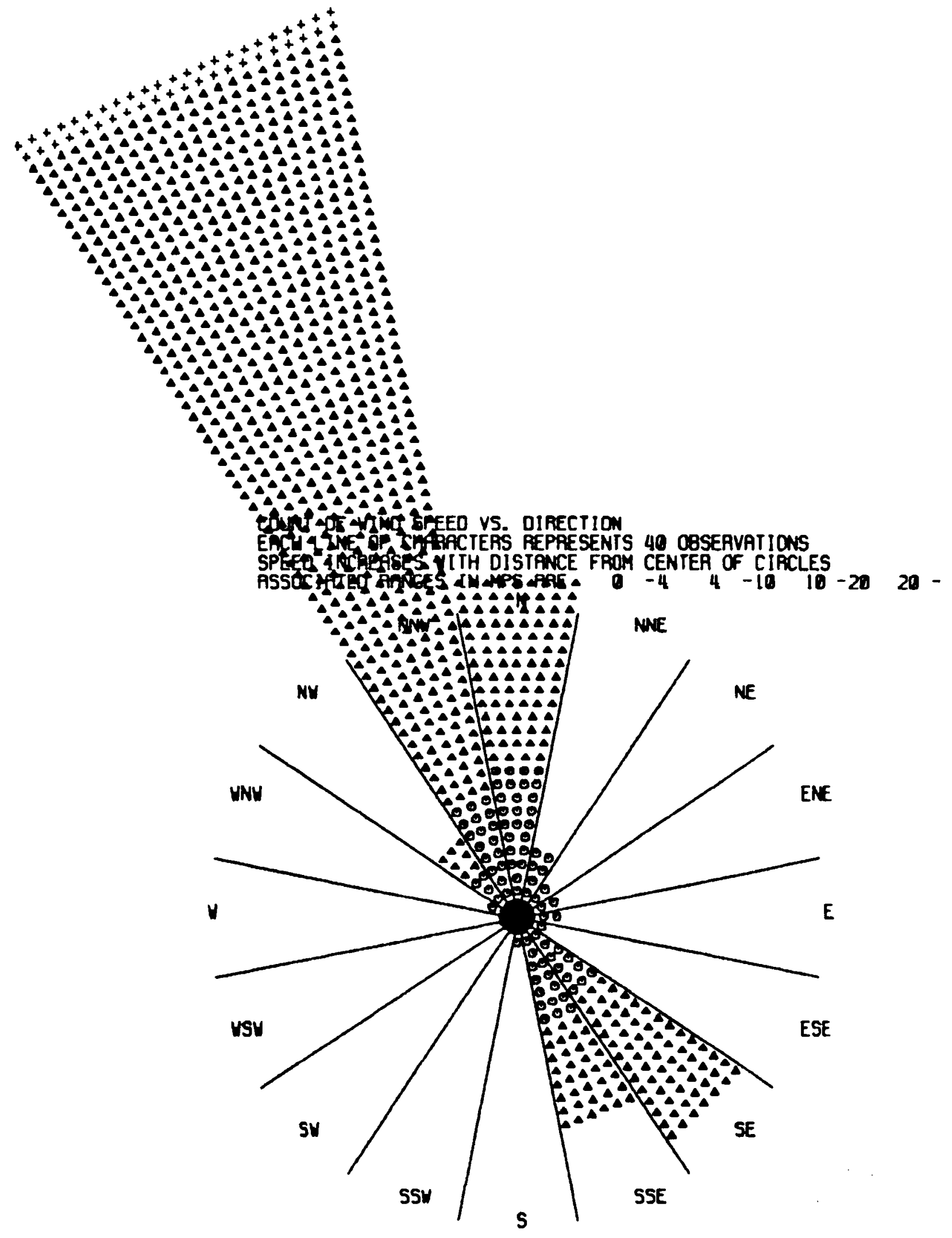

FIGURE 1. (CA) Wind Rose for Lowest Sensor Level 

$+$

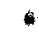

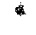


RUSSELL, KANSAS 


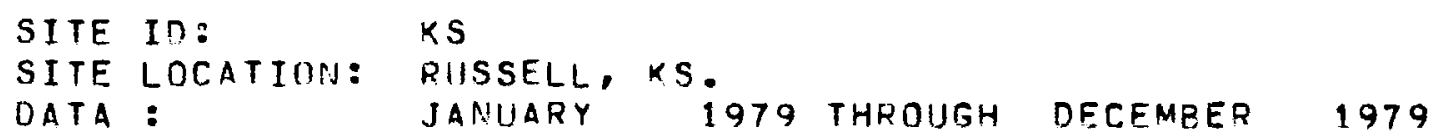

(A) 45.7

$19.1 \quad 345.6 \quad 12 / 11 / 79$

$07: 00$

(B) -999.9

(C) 9.1

$16.4 \quad 336.5 \quad 03 / 23 / 79$

$11: 00$

(C) 14.8

(A) 18.8

(B) -999.9

NOTES:

1. Site eleVATION: 564 METERS AROVE SEA LFVEL.

3. SENSOR LEVEL a NOT AVAILABLE AT SITE KS. 
$\begin{array}{llll}\text { SITE ID: } & \text { KS } & & \\ \text { SITE LOCATION: } & \text { RIJSSELL, KS. } & & \\ \text { DATA : } & \text { JANUARY } & 1979 \text { THROUJH DECEMBER } & 1979\end{array}$

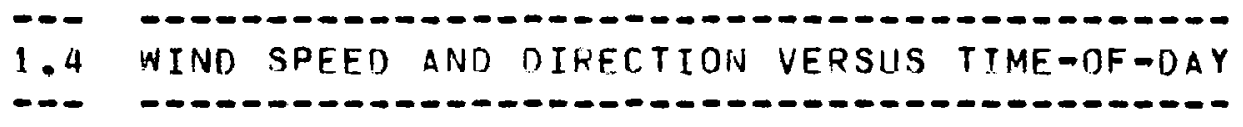

\begin{tabular}{|c|c|c|c|c|c|c|}
\hline \multirow{3}{*}{$\begin{array}{l}\text { TIME } \\
\text { OF DAY }\end{array}$} & \multicolumn{2}{|c|}{ LEVEL A } & & $\begin{array}{l}8 \\
---\end{array}$ & & $\therefore \quad C$ \\
\hline & WS & ND & WS & wo & WS & WD \\
\hline & - & & & & & - \\
\hline $00: 00$ & 7.2 & 124.9 & 0.0 & 0.0 & 4.7 & 145.1 \\
\hline $01: 00$ & 7.0 & 139.6 & 0.0 & $n .0$ & 4.5 & 169.0 \\
\hline $02: 00$ & 7.0 & 143.9 & 0.0 & 0.0 & 4.6 & 182.4 \\
\hline $03: 00$ & 6.9 & 166.0 & 0.0 & 0.0 & 4.5 & 191.0 \\
\hline $04: 00$ & 6.9 & 175.4 & 0.0 & 0.0 & 4.4 & 195.2 \\
\hline $05: 00$ & 6.9 & 190.1 & 0.0 & 0.0 & 4.5 & 200.1 \\
\hline $06: 00$ & 7.0 & 179.0 & 0.0 & 0.0 & 4.5 & 192.6 \\
\hline $07: 00$ & 6.8 & 189.2 & 0.0 & 0.0 & 4.6 & 197.6 \\
\hline $08: 00$ & 6.8 & 193.5 & 0.0 & 0.0 & 5.0 & 201.4 \\
\hline $09: 00$ & 6.9 & 202.4 & 0.0 & 0.0 & 5.5 & 204.9 \\
\hline $10: 00$ & 7.0 & 199.11 & 0.0 & 0.0 & 5.8 & 205.4 \\
\hline $11: 00$ & 7.0 & 205.5 & 0.0 & 0.0 & 5.8 & 208.1 \\
\hline $12: 00$ & 7.1 & 196.6 & 0.0 & 0.0 & 5.9 & 198.8 \\
\hline $13: 00$ & 7.2 & 196.4 & 0.0 & 0.0 & 6.0 & 198.1 \\
\hline $14: 00$ & 7.3 & 182.4 & 0.0 & 0.0 & 6.0 & 185.8 \\
\hline $15: 00$ & 7.1 & 164.0 & 0.0 & 0.0 & 5.9 & 176.5 \\
\hline $16: 00$ & 7.0 & 126.2 & 0.0 & 0.0 & 5.6 & 140.9 \\
\hline $17: 00$ & 7.0 & 103.0 & 0.0 & 0.0 & 5.4 & 112.7 \\
\hline $18: 0 n$ & 6.9 & 98.0 & 0.0 & 0.0 & 5.1 & 107.0 \\
\hline $19: 00$ & 7.0 & 95.9 & 0.0 & 0.0 & 4.8 & 105.2 \\
\hline $20: 0 n$ & 6.9 & 98.1 & 0.0 & 0.0 & 4.5 & 109.1 \\
\hline $21: 00$ & 6.9 & 105.0 & 0.0 & 0.0 & 4.4 & 115.8 \\
\hline $22: 00$ & 7.1 & 115.9 & 0.0 & 0.0 & 4.5 & 130.5 \\
\hline $23: 00$ & 7.2 & 117.6 & 0.0 & 0.0 & 4.6 & 137.6 \\
\hline
\end{tabular}




$\begin{array}{lllll}\text { SITE TD: } & \text { KS } & & & \\ \text { SITE LOCATION: } & \text { RUSSELL, KS } & & \\ \text { DATA : } & \text { JANIIARY } & 1979 & \text { THROUGH DECEMBER } & 1979\end{array}$

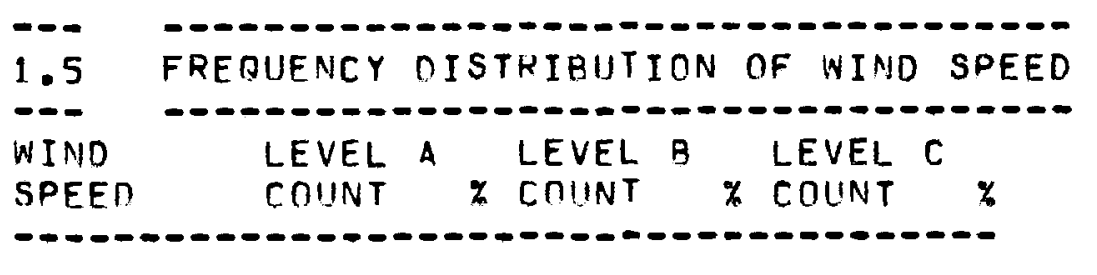

$\begin{array}{rrrrrrr}0.0-0.5 & 4 & 0.1 & 0 & 0.0 & 10 & 0.1 \\ 0.5-1.0 & 43 & 0.6 & 0 & 0.0 & 85 & 1.1 \\ 1.0-1.5 & 91 & 1.2 & 0 & 0.0 & 207 & 2.8 \\ 1.5-2.0 & 124 & 1.7 & 0 & 0.0 & 317 & 4.3 \\ 2.0-2.5 & 190 & 2.6 & 0 & 0.0 & 506 & 6.8 \\ 2.5-3.0 & 240 & 3.2 & 0 & 0.0 & 634 & 8.6 \\ 3.0-3.5 & 278 & 3.7 & 0 & 0.0 & 667 & 9.0 \\ 3.5-4.0 & 308 & 4.1 & 0 & 0.0 & 642 & 8.7 \\ 4.0-4.5 & 433 & 5.8 & 0 & 0.0 & 582 & 7.9 \\ 4.5-5.0 & 386 & 5.2 & 0 & 0.0 & 515 & 7.0 \\ 5.0-5.5 & 449 & 6.0 & 0 & 0.0 & 487 & 6.6 \\ 5.5-6.0 & 484 & 6.5 & 0 & 0.0 & 445 & 6.0 \\ 6.0-6.5 & 486 & 6.5 & 0 & 0.0 & 375 & 5.1 \\ 6.5-7.0 & 486 & 6.5 & 0 & 0.0 & 334 & 4.5 \\ 7.0-7.5 & 412 & 5.5 & 0 & 0.0 & 302 & 4.1 \\ 7.5-8.0 & 399 & 5.4 & 0 & 0.0 & 264 & 3.6 \\ 8.0-8.5 & 401 & 5.4 & 0 & 0.0 & 208 & 2.8 \\ 8.5-9.0 & 349 & 4.7 & 0 & 0.0 & 174 & 2.4 \\ 9.0-9.5 & 328 & 4.4 & 0 & 0.0 & 147 & 2.0 \\ 9.5-10.0 & 289 & 3.9 & 0 & 0.0 & 123 & 1.7 \\ 10.0-11.0 & 455 & 6.1 & 0 & 0.0 & 171 & 2.3 \\ 11.0-12.0 & 272 & 3.7 & 0 & 0.0 & 102 & 1.4 \\ 12.0-13.0 & 194 & 2.6 & 0 & 0.0 & 41 & 0.6 \\ 13.0-14.0 & 156 & 2.1 & 0 & 0.0 & 36 & 0.5 \\ 14.0-15.0 & 73 & 1.0 & 0 & 0.0 & 17 & 0.2 \\ 15.0-16.0 & 43 & 0.6 & 0 & 0.0 & 8 & 0.1 \\ 16.0-17.0 & 23 & 0.3 & 0 & 0.0 & 1 & 0.0 \\ 17.0-18.0 & 21 & 0.3 & 0 & 0.0 & 0 & 0.0 \\ 18.0-19.0 & 8 & 0.1 & 0 & 0.0 & 0 & 0.0 \\ 19.0-20.0 & 1 & 0.0 & 0 & 0.0 & 0 & 0.0 \\ 20.0-21.0 & 0 & 0.0 & 0 & 0.0 & 0 & 0.0 \\ >21.0 & 0 & 0.0 & 0 & 0.0 & 0 & 0.0\end{array}$

LEVEL A LEVEL 8 LEVEL C

$$
\begin{array}{lll}
84.8 & 0.0 & 84.5
\end{array}
$$




$\begin{array}{lllll}\text { SITE ID: } & \text { KS } & & & \\ \text { SITE LOCATION: } & \text { RUSSELL, KS } & & \\ \text { DATA : } & \text { JAMIIARY } & \text { I979 THROUGH DECEMBER } & 1979\end{array}$

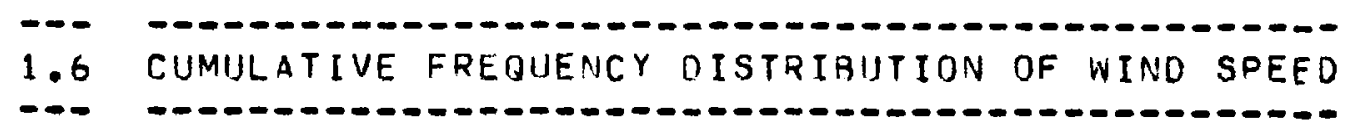

\begin{tabular}{|c|c|c|c|c|c|c|}
\hline & LEV & $\therefore \quad A$ & LEV & B & LEV & L C C \\
\hline$-\infty-\infty$ & 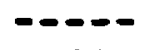 & $-----\infty$ & $\ldots \ldots$ & - - - & 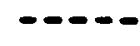 & \\
\hline $\begin{array}{l}\text { WIND } \\
\text { SPEED }\end{array}$ & $\begin{array}{c}\text { CFD } \\
(A B S)\end{array}$ & $\begin{array}{l}\text { CFD } \\
(\%)\end{array}$ & $\begin{array}{c}C F \cap \\
(A B S)\end{array}$ & $\begin{array}{l}C F D \\
(z)\end{array}$ & $\begin{array}{c}C F D \\
(A B S)\end{array}$ & $\begin{array}{l}\text { CFD } \\
(y)\end{array}$ \\
\hline---- & $--\infty$ & ---- & ---- & $-\infty-\infty$ & $\ldots .$. & $\cdots-\infty$ \\
\hline 0.5 & 4 & 0.05 & 0 & 0.00 & 10 & 0.1 \\
\hline 1.0 & 47 & 0.63 & 0 & 0.00 & 95 & 1.28 \\
\hline 1.5 & 138 & 1.86 & 0 & 0.00 & 302 & 4.08 \\
\hline 2. 0 & 262 & 3.53 & 0 & 0.00 & 619 & 8.36 \\
\hline 2.5 & 452 & 6.09 & 0 & 0.00 & 1125 & 15.20 \\
\hline 3.0 & 692 & 9.32 & 0 & 0.00 & 1759 & 23.77 \\
\hline 3.5 & 970 & 13.06 & 0 & 0.00 & 2426 & 32.78 \\
\hline 4.0 & 1278 & 17.21 & 0 & 0.00 & 3068 & 41.46 \\
\hline 4.5 & 1711 & 23.04 & 0 & 0.00 & 3650 & 49.32 \\
\hline 5.0 & 2097 & 28.24 & 0 & 0.00 & 4165 & 56.28 \\
\hline 5.5 & 2546 & 34.28 & 0 & 0.00 & 4652 & 62.86 \\
\hline 6.0 & 3030 & 40.80 & 0 & 0.00 & 5097 & 68.89 \\
\hline 6.5 & 3516 & 47.35 & 0 & 0.00 & 5472 & 73.95 \\
\hline 7.0 & 4002 & 53.89 & 0 & 0.00 & 5806 & 78.46 \\
\hline 7.5 & 4414 & 59.44 & 0 & 0.00 & 6108 & 82.54 \\
\hline 8.0 & 4813 & 64.81 & 0 & 0.00 & 6372 & 86.11 \\
\hline 8.5 & 5214 & 70.21 & 0 & 0.00 & 6580 & 98.92 \\
\hline 9.0 & 5563 & 74.91 & 0 & 0.00 & 6754 & 91.27 \\
\hline 9.5 & 5891 & 79.33 & 0 & 0.00 & 6901 & 93.26 \\
\hline 10.0 & 6180 & 33.22 & 0 & 0.00 & 7024 & 94.92 \\
\hline 11.0 & 6635 & 89.35 & 0 & 0.00 & 7195 & 97.23 \\
\hline 12.0 & 6907 & 93.01 & n & 0.00 & 7297 & 98.61 \\
\hline 13.0 & 7101 & 95.62 & 0 & 0.00 & 7338 & 99.16 \\
\hline 14.0 & 7257 & 97.72 & 0 & 0.00 & 7374 & 99.65 \\
\hline 15.0 & 7330 & 98.71 & 0 & 0.00 & 7391 & 99.88 \\
\hline 16.0 & 7373 & 99.29 & 0 & 0.00 & 7399 & 99.99 \\
\hline 17.0 & 7396 & 99.60 & 0 & 0.00 & 7400 & 100.00 \\
\hline 18.0 & 7417 & 99.88 & 0 & 0.00 & 7400 & 100.00 \\
\hline 19.0 & 7425 & 99.99 & 0 & 0.00 & 7400 & 100.00 \\
\hline 20.0 & 7426 & 100.00 & 0 & 0.00 & 7400 & 100.00 \\
\hline 21.0 & 7426 & 100.00 & 0 & 0.00 & 7400 & 100.00 \\
\hline 21.0 & 7426 & 100.00 & 0 & 0.00 & 7400 & 100.00 \\
\hline
\end{tabular}



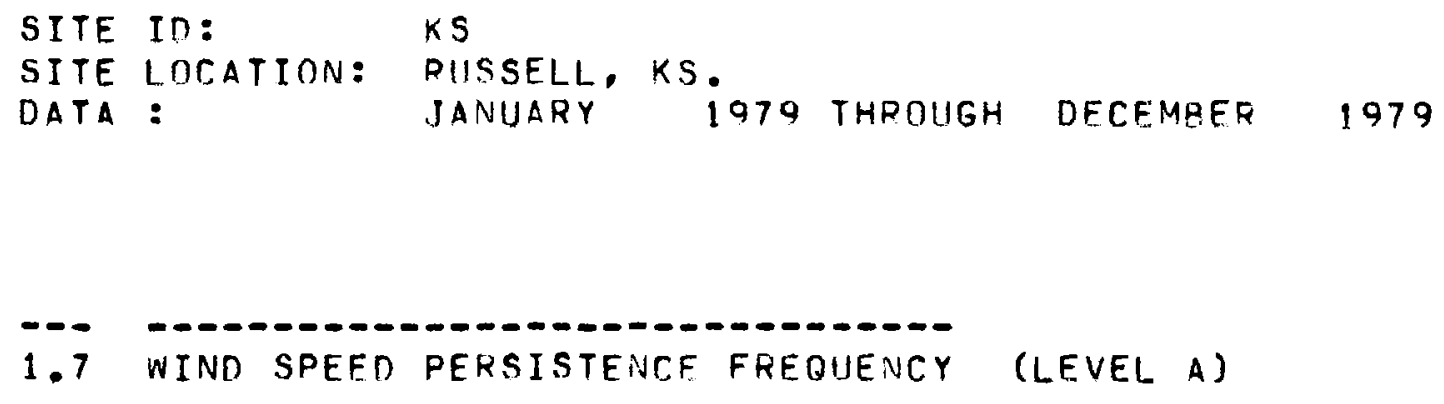

WIND SPEED PERSISTENCF FREQUENCY

$-0$
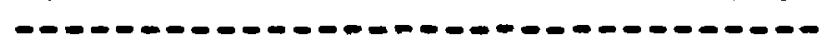

(LEVEL A)

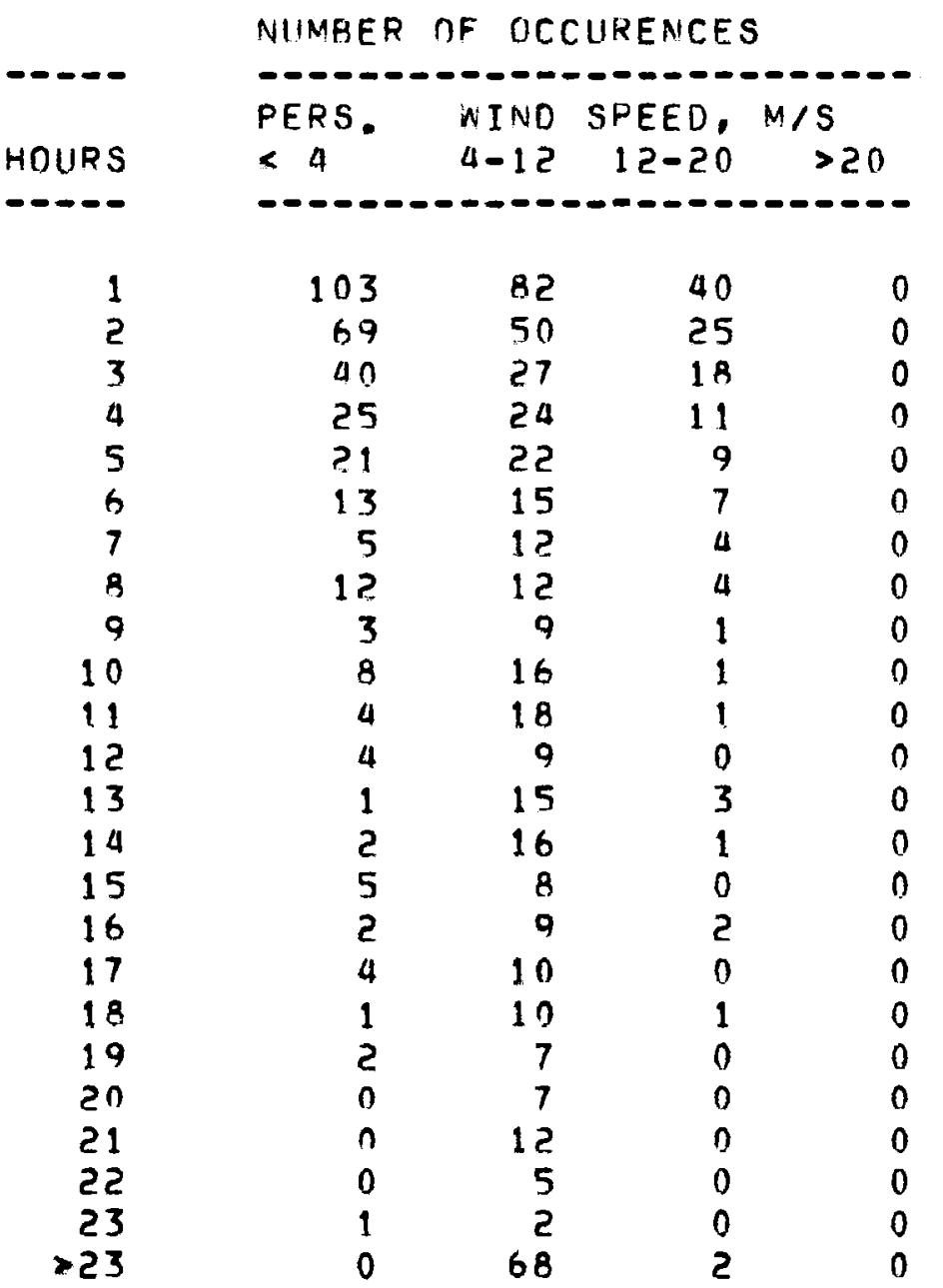




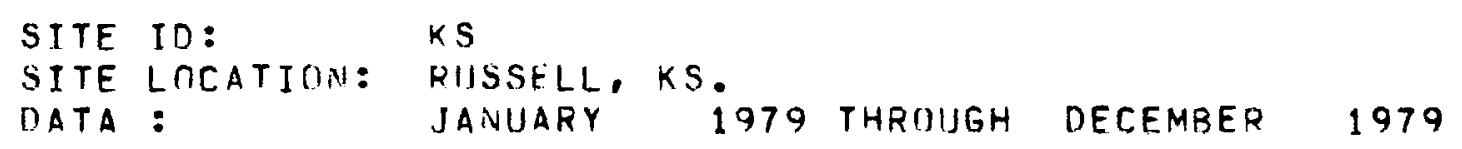

$\begin{array}{llllllll}\text { N } & 0.00 & 0.00 & 0.16 & 0.00 & 7.02 & 0.00 & 6.59 \\ \text { NNE } & 0.00 & 0.00 & 0.15 & 0.00 & 6.25 & 0.00 & 5.78 \\ \text { NE } & 0.00 & 0.00 & 0.16 & 0.00 & 5.40 & 0.00 & 5.19 \\ \text { ENE } & 0.00 & 0.00 & 0.17 & 0.00 & 4.44 & 0.00 & 4.45 \\ \text { E } & 0.00 & 0.00 & 0.24 & 0.00 & 4.24 & 0.00 & 3.81 \\ \text { ESE } & 0.00 & 0.00 & 0.29 & 0.00 & 4.09 & 0.00 & 4.23 \\ \text { SE } & 0.00 & 0.00 & 0.29 & 0.00 & 9.01 & 0.00 & 7.28 \\ \text { SSE } & 0.00 & 0.00 & 0.22 & 0.00 & 14.23 & 0.00 & 12.66 \\ \text { S } & 0.00 & 0.00 & 0.19 & 0.00 & 11.24 & 0.00 & 13.35 \\ \text { SSW } & 0.00 & 0.00 & 0.20 & 0.00 & 5.39 & 0.00 & 6.08 \\ \text { SW } & 0.00 & 0.00 & 0.29 & 0.00 & 3.22 & 0.00 & 4.64 \\ \text { WSW } & 0.00 & 0.00 & 0.31 & 0.00 & 3.02 & 0.00 & 3.47 \\ \text { W } & 0.00 & 0.00 & 0.29 & 0.00 & 3.02 & 0.00 & 3.20 \\ \text { WNW } & 0.00 & 0.00 & 0.25 & 0.00 & 4.26 & 0.00 & 4.34 \\ \text { NW } & 0.00 & 0.00 & 0.20 & 0.00 & 7.43 & 0.00 & 6.74 \\ \text { NNW } & 0.00 & 0.00 & 0.11 & 0.00 & 7.84 & 0.00 & 7.41\end{array}$

NOTES:

$$
\begin{aligned}
& \text { WS (UP) Z (UP) } \\
& \text { 1. } \frac{W S(L O)}{Z(L O)} \\
& \begin{array}{l}
\text { 2. } A L P H A=\frac{\operatorname{LOG}(W S(U P) / W S(L O))}{\text { LOG }(Z(U P) / Z(L O))} \quad \text { WHERE; Z=ELEVATION } \\
\text { WS =WIND SPEED }
\end{array}
\end{aligned}
$$


COUNT OF VIND SPEED VS. DIRECTION

EACH LINE OF CHARACTERS REPRESENTS 40 OBSERVATIONS

SPEED INCREASES WITH DISTANCE FROM CENTER OF CIRCLES

ASSOCIATED RANGES IN MPS ARE $00-4 \quad 4-10 \quad 10-20 \quad 20-$

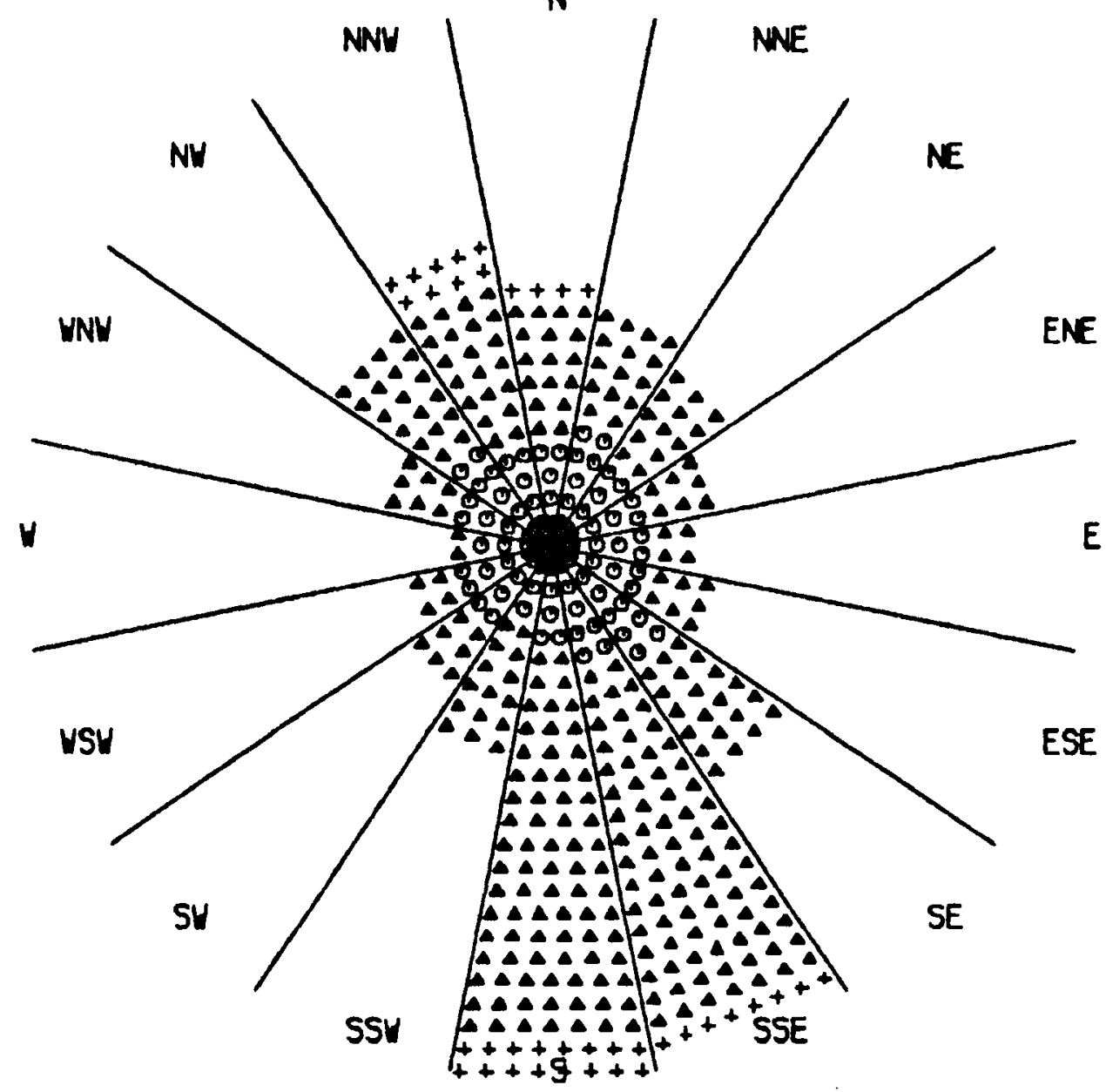

FIGURE 1. (KS) Wind Rose for Lowest Sensor Leve1 


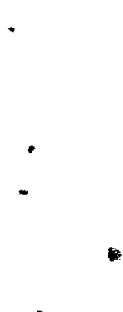


SAN GORGONIO PASS, CALIFORNIA 
SITE ID: $\quad$ SG

SITE LOCATION: SAN GORGUNIO PASS,CA

DATA : JANUARY 1979 THROUGH DECEMBER 1979

1.1 SENSOR PERFORMANCE

TOTAL POSSIBLE SAMPLES: 8760

$\begin{array}{lcc}\text { SENSOR } & \text { \% DN-LINE } & \% \text { RECOVERED } \\ \text { WS (A) } & 100.0 & 90.0 \\ \text { WD(A) } & 100.0 & 89.5 \\ \text { WS (C) } & 100.0 & 90.4 \\ \text { WD (C) } & 100.0 & 90.7\end{array}$

1.2 ANNUAL MEANS AND STANDARD DEVIATIONS

1.-.-.-

$\begin{array}{cccc}\text { HEIGHT } & \text { MEAN } & \text { MEAN } & \text { STD DEV } \\ \text { (METERS) } & \text { WS } & \text { WD } & \text { SPEED }\end{array}$

$\begin{array}{lrrrrr}\text { SITE DATA } & \text { (A) } & 45.7 & 7.2 & 239.1 & 5.2 \\ & \text { (C) } & 9.1 & 5.6 & 251.9 & 3.8\end{array}$

1.3 MAXIMUM WIND SPEED

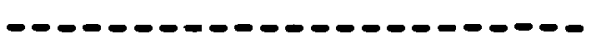

ELEVATION

(METERS)

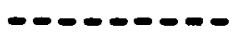
(A) 45.7
24.3
228.6
$05 / 06 / 79$
$234.8 \quad 05 / 06 / 79$
18.7
$18: 00$
(B) -999.9
(C) 18.7
(C) 9.1
$18: 00$
(A) 24.3

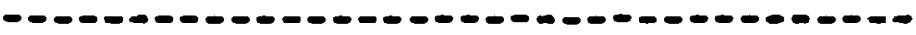

NOTES:

1. SITE ELEVATION: 329 METERS abOVE SEA LEVEL.

3. SENSOR LEVEL B NOT AVAILABLE AT SITE SG. 

SITE ID:
SG
SITE LOCATION:
UATA :
SAN GURGUNIO PASS, CA
JANUARY
1979 THKDUGH
DECEMBER
1979

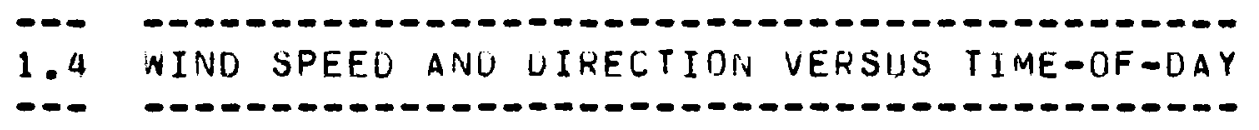

\begin{tabular}{|c|c|c|c|c|c|c|}
\hline \multirow{2}{*}{ TIME } & \multicolumn{2}{|c|}{ LEVEL A } & \multicolumn{2}{|c|}{ LEVEL $B$} & \multicolumn{2}{|c|}{ LEVEL C } \\
\hline & WS & WD & wS & WD & WS & WD \\
\hline$----\infty$ & & & -1 & --- & & $--\infty$ \\
\hline $00: 00$ & 7.4 & 259.1 & 0.0 & 0.0 & 5.7 & 267.9 \\
\hline $01: 00$ & 7.3 & 261.2 & 0.0 & 0.0 & 5.5 & 270.6 \\
\hline $02: 00$ & 7.1 & 265.7 & 0.0 & 0.0 & 5.5 & 275.2 \\
\hline $03: 00$ & 6.8 & 265.4 & 0.0 & 0.0 & 5.2 & 275.5 \\
\hline $04: 00$ & 6.8 & 264.9 & 0.0 & 0.0 & 5.2 & 275.5 \\
\hline $05: 00$ & 6.6 & 267.9 & 0.0 & 0.0 & 5.0 & 277.5 \\
\hline $06: 00$ & 6.2 & 271.9 & 0.0 & 0.0 & 4.7 & 281.7 \\
\hline $07: 00$ & 5.9 & 275.5 & 0.0 & 0.0 & 4.5 & 279.6 \\
\hline $08: 00$ & 5.7 & 251.0 & 0.0 & 0.0 & 4.5 & 247.8 \\
\hline $09: 00$ & 5.9 & 170.4 & 0.0 & 0.0 & 4.7 & 169.9 \\
\hline $10: 00$ & 6.1 & 159.9 & 0.0 & 0.0 & 4.9 & 162.0 \\
\hline $11: 00$ & 6.5 & 157.6 & 0.0 & 0.0 & 5.2 & 158.9 \\
\hline $12: 00$ & 6.8 & 160.5 & 0.0 & 0.0 & 5.5 & 161.1 \\
\hline $13: 00$ & 7.1 & 163.1 & 0.0 & 0.0 & 5.7 & 164.1 \\
\hline $14: 00$ & 7.4 & 172.1 & 0.0 & 0.0 & 5.8 & 173.4 \\
\hline $15: 00$ & 7.6 & 182.9 & 0.0 & 0.0 & 6.0 & 183.7 \\
\hline $16: 00$ & 7.8 & 194.9 & 0.0 & 0.0 & 6.0 & 205.7 \\
\hline $17: 00$ & 8.2 & 222.2 & 0.0 & 0.0 & 6.2 & 244.5 \\
\hline $18: 00$ & 8.5 & 239.9 & 0.0 & 0.0 & 6.4 & 254.6 \\
\hline $19: 00$ & 8.7 & 245.7 & 0.0 & 0.0 & 6.5 & 257.5 \\
\hline $20: 00$ & B. 5 & 250.7 & 0.0 & 0.0 & 6.5 & 258.1 \\
\hline $21: 00$ & 8.4 & 249.0 & 0.0 & 0.0 & 6.3 & 259.9 \\
\hline $22: 00$ & 8.0 & 253.0 & 0.0 & 0.0 & 6.0 & 262.8 \\
\hline $23: 00$ & 7.7 & 256.9 & 0.0 & 0.0 & 5.8 & 265.3 \\
\hline
\end{tabular}


SITE ID: $\quad$ SG

SITE LOCATION: SAN GOKGUNIO PASS,CA

DATA : JANUARY 1979 THRDUGH DECEMBER 1979

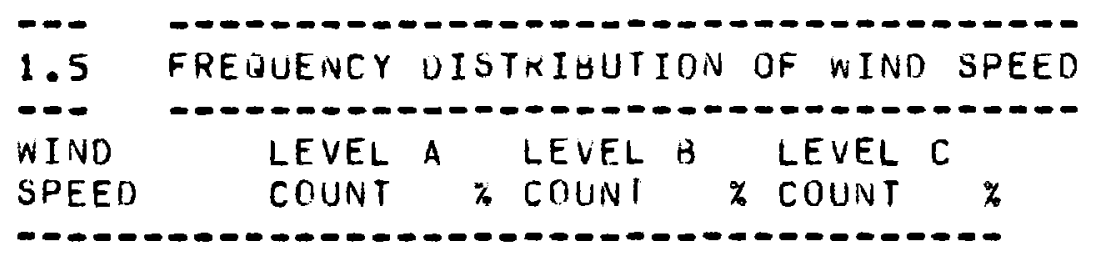

$\begin{array}{rrrrrrr}0.0-0.5 & 89 & 1.1 & 0 & 0.0 & 19 & 0.2 \\ 0.5-1.0 & 370 & 4.7 & 0 & 0.0 & 237 & 3.0 \\ 1.0-1.5 & 493 & 6.3 & 0 & 0.0 & 530 & 6.7 \\ 1.5-2.0 & 496 & 6.3 & 0 & 0.0 & 727 & 9.2 \\ 2.0-2.5 & 497 & 6.3 & 0 & 0.0 & 673 & 0.5 \\ 2.5-3.0 & 429 & 5.4 & 0 & 0.0 & 642 & 8.1 \\ 3.0-3.5 & 372 & 4.7 & 0 & 0.0 & 463 & 5.8 \\ 3.5-4.0 & 332 & 4.2 & 0 & 0.0 & 383 & 4.8 \\ 4.0-4.5 & 236 & 3.0 & 0 & 0.0 & 300 & 3.8 \\ 4.5-5.0 & 228 & 2.9 & 0 & 0.0 & 299 & 3.8 \\ 5.0-5.5 & 220 & 2.8 & 0 & 0.0 & 268 & 3.4 \\ 5.5-0.0 & 176 & 2.2 & 0 & 0.0 & 224 & 2.8 \\ 6.0-6.5 & 186 & 2.4 & 0 & 0.0 & 194 & 2.5 \\ 6.5-7.0 & 151 & 1.9 & 0 & 0.0 & 233 & 2.9 \\ 7.0-7.5 & 176 & 2.2 & 0 & 0.0 & 233 & 2.9 \\ 7.5-8.0 & 188 & 2.4 & 0 & 0.0 & 260 & 3.3 \\ 8.0-0.5 & 164 & 2.1 & 0 & 0.0 & 249 & 3.1 \\ 8.5-9.0 & 181 & 2.3 & 0 & 0.0 & 244 & 3.1 \\ 9.0-9.5 & 184 & 2.3 & 0 & 0.0 & 261 & 3.3 \\ 9.5-10.0 & 179 & 2.3 & 0 & 0.0 & 257 & 3.2 \\ 10.0-11.0 & 378 & 4.8 & 0 & 0.0 & 449 & 5.7 \\ 11.0-12.0 & 435 & 5.5 & 0 & 0.0 & 314 & 4.0 \\ 12.0-13.0 & 362 & 4.6 & 0 & 0.0 & 196 & 2.5 \\ 13.0-14.0 & 386 & 4.9 & 0 & 0.0 & 104 & 1.3 \\ 14.0-15.0 & 310 & 3.9 & 0 & 0.0 & 66 & 0.8 \\ 15.0-16.0 & 225 & 2.9 & 0 & 0.0 & 40 & 0.5 \\ 16.0-17.0 & 141 & 1.8 & 0 & 0.0 & 35 & 0.4 \\ 17.0-18.0 & 107 & 1.4 & 0 & 0.0 & 14 & 0.2 \\ 18.0-19.0 & 62 & 0.8 & 0 & 0.0 & 3 & 0.0 \\ 19.0-20.0 & 42 & 0.5 & 0 & 0.0 & 0 & 0.0 \\ 20.0-21.0 & 28 & 0.4 & 0 & 0.0 & 0 & 0.0 \\ >21.0 & 59 & 0.7 & 0 & 0.0 & 0 & 0.0\end{array}$

LEVEL A LEVEL 8 LEVEL C

$90.0 \quad 0.0 \quad 90.4$ 
$\begin{array}{ll}\text { SITE ID: } & \text { SG } \\ \text { SITE LOCATION: } & \text { SAN GOKGUNIO PASS,CA }\end{array}$

DATA : JANUARY 1979 THRDUGH DECEMBEF 1979

1.6 CUMULATIVE FREQUENCY DISTRIBUTION OF WIMD SPEED

LEVEL A

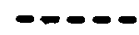

WIND

SPEED

- - -

0.5

1.0

1.5

2.0

2. 5

3.0

3.5

4.0

4.5

5.0

5.5

6.0

6.5

7.0

7.5

8.0

8.5

9.0

9.5

10.0

11.0

12.0

13.0

14.0

15.0

16.0

17.0

18.0

19.0

20.0

21.0

$\times 21.0$
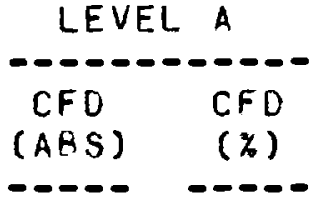

$89 \quad 1.13$

4595.82

$952 \quad 12.08$

$\begin{array}{lll}1448 & 18.37\end{array}$

194524.68

237430.12

$2746 \quad 34.84$

$3078 \quad 39.05$

331442.05

$3542 \quad 44.94$

$3762 \quad 47.73$

$3938 \quad 49.96$

$4124 \quad 52.32$

$4275 \quad 54.24$

$4451 \quad 56.47$

$4639 \quad 58.86$

$4803 \quad 00.94$

$4984 \quad 63.23$

$\begin{array}{ll}5168 & 65.57\end{array}$

$5347 \quad 67.84$

$5725 \quad 72.63$

$6160 \quad 78.15$

$6522 \quad 82.75$

6908

7218

7443

7584

7691

7753

7795

7823

7882

87.64

91.58

94.43

96.22

97.58

98.36

98.90

99.25

100.00
LEVEL $B$

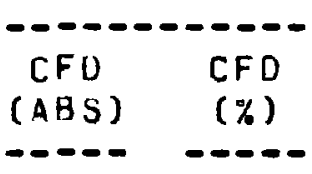

0

$0 \quad 0.00$

$0 \quad 0.00$

$0 \quad 0.00$

$0 \quad 0.00$

$0 \quad 0.00$

$0 \quad 0.00$

$0 \quad 0.00$

$0 \quad 0.00$

$0 \quad 0.00$

$0 \quad 0.00$

$0 \quad 0.00$

$0 \quad 0.00$

$0 \quad 0.00$

$0 \quad 0.00$

$0 \quad 0.00$

$0 \quad 0.00$

$0 \quad 0.00$

$0 \quad 0.00$

$0 \quad 0.00$

$0 \quad 0.00$

$0 \quad 0.00$

$0 \quad 0.00$
0.00

0.00

0.00

0.00

0.00

0.00

0.00

0.00

0.00
LEVEL C

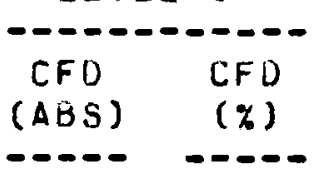

190.24

$256 \quad 3.23$

$\begin{array}{ll}786 & 9.93\end{array}$

$1513 \quad 19.11$

$2186 \quad 27.61$

$2828 \quad 35.72$

329141.57

$3674 \quad 46.41$

397450.20

$4273 \quad 53.97$

$4541 \quad 57.36$

$4765 \quad 60.19$

$4959 \quad 62.64$

$5192 \quad 65.58$

$5425 \quad 68.52$

568571.81

$5934 \quad 74.95$

$6178 \quad 78.03$

$6439 \quad 81.33$

$6696 \quad 84.58$

$7145 \quad 90.25$

$7459 \quad 94.21$

765596.69

775998.00

$7825 \quad 98.84$

$7865 \quad 99.34$

$7900 \quad 99.79$

$7914 \quad 99.96$

7917100.00

7917100.00

7917100.00

7917100.00 
SITE IO: SG

SITE LOCATION: SAN GORGONIO PASS,CA

DATA : JANUARY 1979 THROUGH DECEMBER 1979

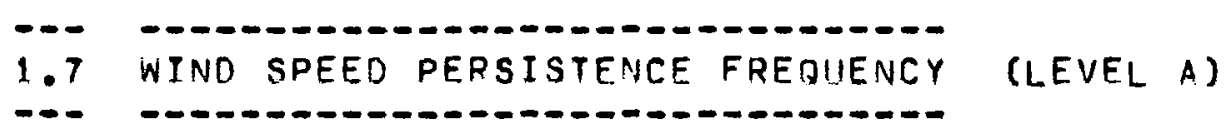

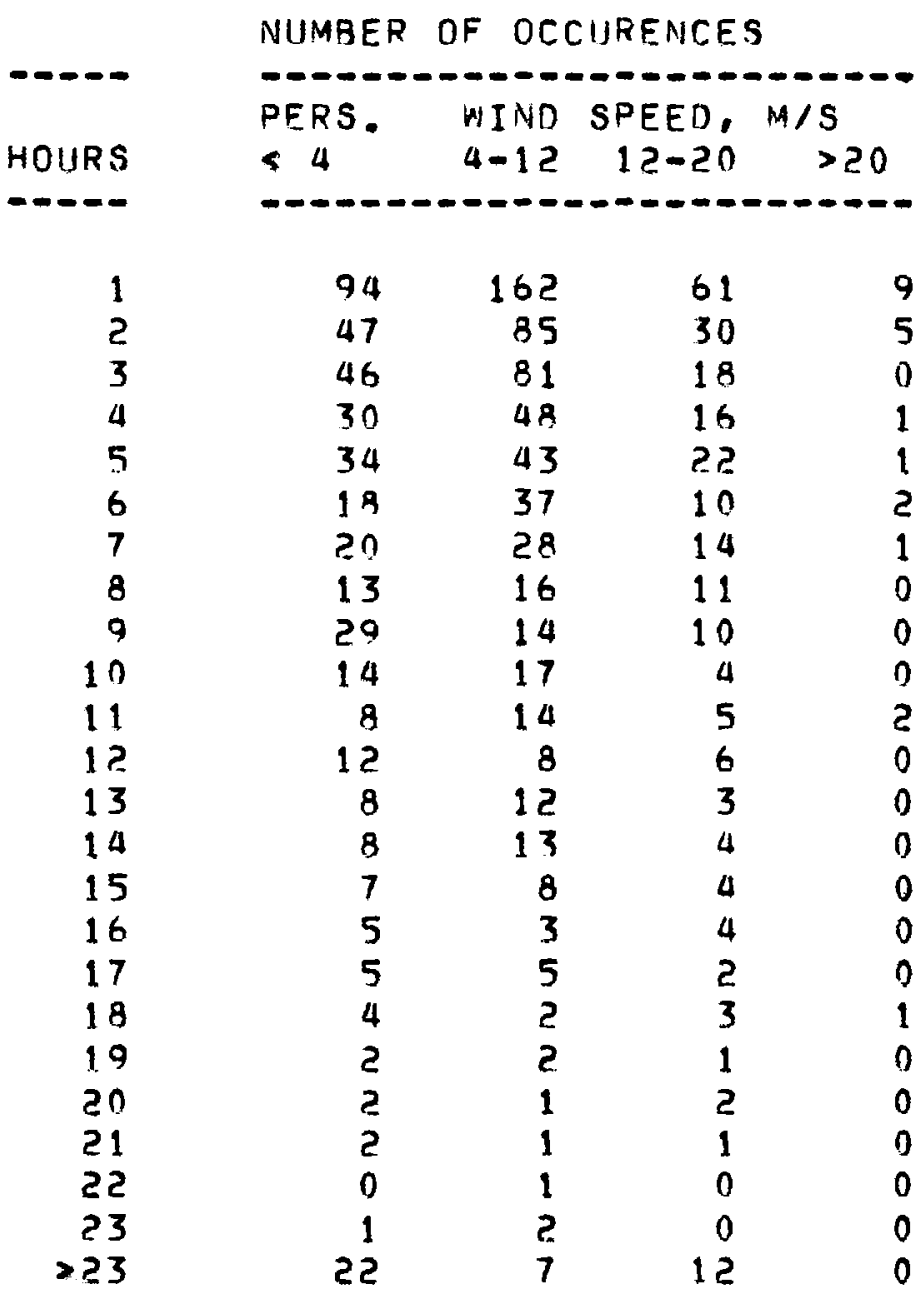


$\begin{array}{ll}\text { SITE IO: } & \text { SG } \\ \text { SITE LOCATION: } & \text { SAN GORGONIO PASS,CA }\end{array}$

DATA : JANUARY 1979 THROUGH DECEMRER 1979

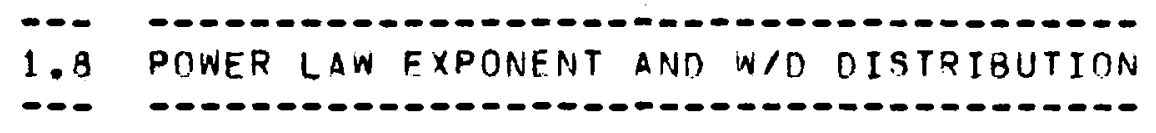

WIND DIR

(ELEV A)

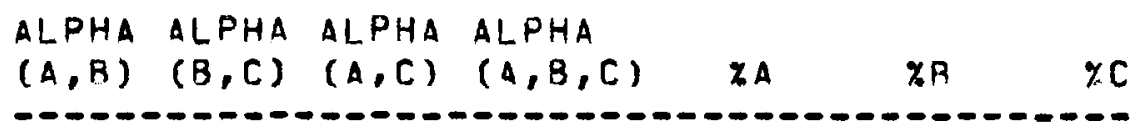

$\begin{array}{lrrrrrrr}\text { N } & 0.00 & 0.00 & 0.14 & 0.00 & 2.36 & 0.00 & 2.06 \\ \text { NNE } & 0.00 & 0.00 & 0.03 & 0.00 & 2.04 & 0.00 & 1.71 \\ \text { NE } & 0.00 & 0.00 & -0.02 & 0.00 & 1.85 & 0.00 & 1.50 \\ \text { ENE } & 0.00 & 0.00 & -0.01 & 0.00 & 2.35 & 0.00 & 1.67 \\ \text { E } & 0.00 & 0.00 & 0.10 & 0.00 & 5.85 & 0.00 & 4.80 \\ \text { ESE } & 0.00 & 0.00 & 0.09 & 0.00 & 7.75 & 0.00 & 7.24 \\ \text { SE } & 0.00 & 0.00 & -0.14 & 0.00 & 2.82 & 0.00 & 2.89 \\ \text { SSE } & 0.00 & 0.00 & -0.30 & 0.00 & 1.69 & 0.00 & 1.52 \\ \text { S } & 0.00 & 0.00 & -0.05 & 0.00 & 1.73 & 0.00 & 1.17 \\ \text { SSN } & 0.00 & 0.00 & 0.07 & 0.00 & 2.80 & 0.00 & 2.34 \\ \text { SW } & 0.00 & 0.00 & 0.19 & 0.00 & 24.47 & 0.00 & 17.89 \\ \text { WSW } & 0.00 & 0.00 & 0.13 & 0.00 & 24.18 & 0.00 & 27.14 \\ \text { N } & 0.00 & 0.00 & 0.06 & 0.00 & 4.31 & 0.00 & 6.16 \\ \text { WNW } & 0.00 & 0.00 & 0.06 & 0.00 & 3.76 & 0.00 & 10.21 \\ \text { NW } & 0.00 & 0.00 & 0.19 & 0.00 & 7.26 & 0.00 & 9.51 \\ \text { NNW } & 0.00 & 0.00 & 0.09 & 0.00 & 3.67 & 0.00 & 2.17\end{array}$

NOTES:

$$
\text { 1. } \frac{W S(U P)}{W S(L O)}=\frac{Z(U P)}{Z(L O)}
$$

$$
\begin{aligned}
& \text { 2. ALPHA }=\frac{\operatorname{LOG}(W S(U P) / W S(L O))}{\operatorname{LOG}(Z(U P) / Z(L O))} \quad \text { WHERE; } \quad \text { Z ELLEVATION } \\
& \text { WS WWIND SPEED }
\end{aligned}
$$




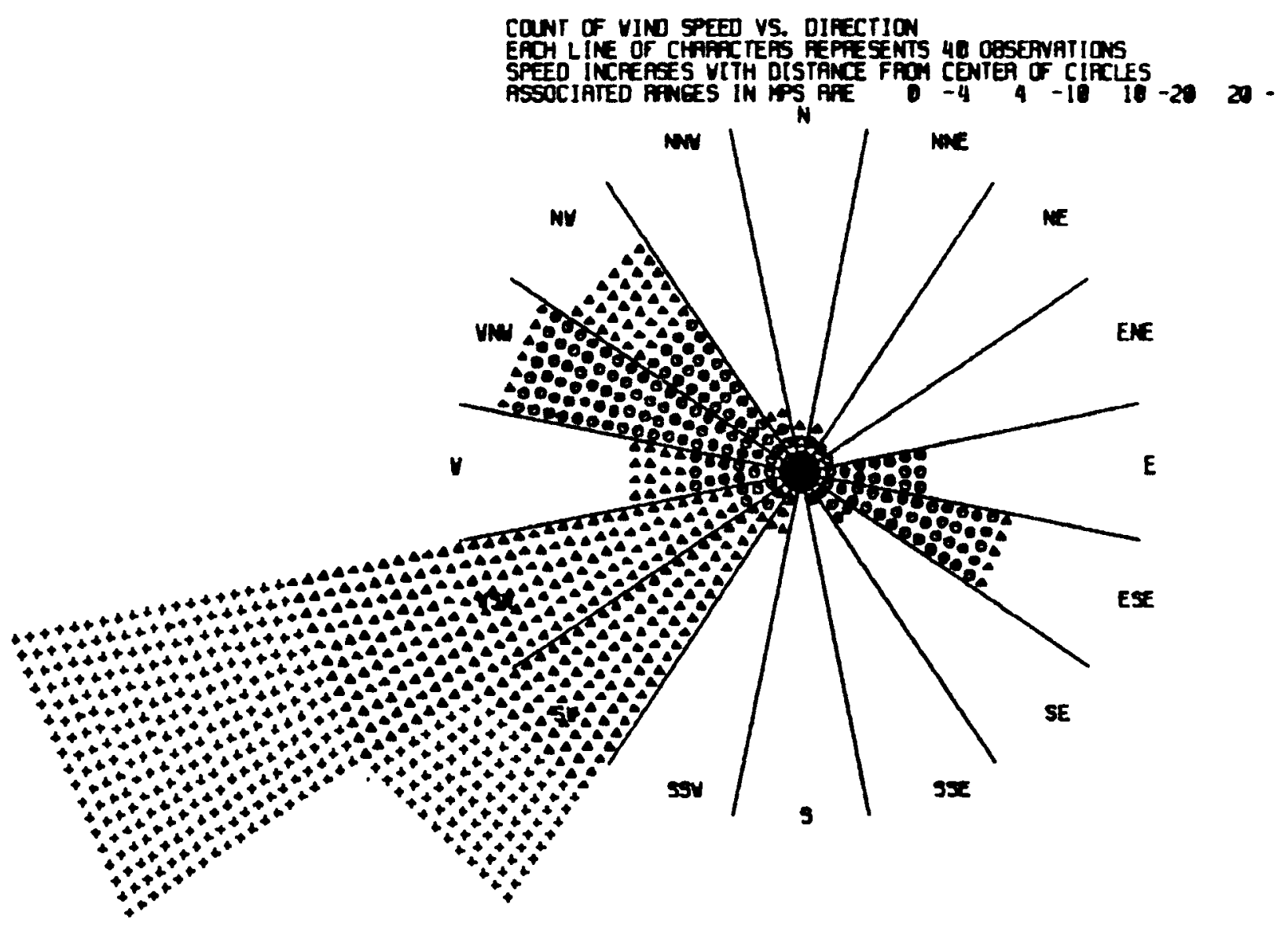

FIGURE 1. (SG) Wind Rose for Lowest Sensor Leve1 


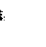




\section{DISTRIBUT ION}

No. of

Copies

\section{OFFSITE}

A. A. Churm

DOE Chicago Patent Group

$9800 \mathrm{~S}$. Cass Avenue

Argonne, IL 60439

Dr. Carl Asliden

Wind Energy Systems Division

600 E. Street N.W.

Washington, DC 20545

George P. Tennyson

Department of Energy

Albuquerque Operations office

P.0. Box 5400

Albuquerque, NM 87115

27 . DOE Technical Information Center

Ronald L. Thomas

NASA-Lewis Research Center

Mail Stop 500-202

21000 Brookpark Road

Cleveland, $\mathrm{OH} 44135$

Mr. Kenneth Ladd

Southwest Public Service Company

P.0. Box 1261

Amarillo, TX 79170

Mr. Merrill E. Slate

Block Island Power Company

P.0. Box 518

Block Island, RI 02807

Mr. Norman Sanesi

Portland General Electric Co.

121 SW Salmon St.

Portland, OR 97204

Mr. Grant Ayers

Blue Ridge Electric Corporation

1216 Blowing Rock Blvd., N.E.

Lenoir, NC 28645
No.. of

Copies

Mr. El i Garcia

City Manager

Clayton, NM 88415

Mr. John Slagle

Alaska Busse11 Electric Company

P.0. Box 4-1325

Anchorage, AK 99509

Mr. Orlando Anglero

Puerto Rico Electric Power Authority

GPO Box 4267

San Juan, PR 00936

Mr. George Leary

City of Holyoke

Gas and Electric Department

70 Suffolk Street

Holyoke, MA 01040

Mr. Jeffery Nelson

East River Electric Power Co-op, Inc.

Madison, SD 57042

Mr. Ed Hamilton

Central Nebraska Publ ic Power and Irrigation District Inc.

P. 0 . Box 356

Holdrege, NB 68949

Mr. Bruce E. Humenik

Senior Research and Development Engineer

Long Island Lighting Company

175 East 0ld Country Road

Hicksville, NY 11801

Mr. Andrew Matura

Long Island Lighting Company

175 East 0ld Company Road

Hicksville, NY 11801 
$\mathrm{Mr}$. Ron Calcaterra Consumers Power Co. 212 W. Michigan Ave. Jackson, MI 49201

Mr. Robert Scheffler Southern California Edison P.0. Box 800

Rosemead, CA 91770

Mr. Earl Homewood

City of Russell

P.0. Box 112

Russe11, KS 67665

Mr. Tom Hillesland

Pacific Gas and Electric Co. Department of Engineering Research 3400 Crow Canyon Road

San Ramon, CA 94583
J. V. Ramsdell

D. S. Renne' (5)

W. F. Sandusky (5)

H. L. Wegley

L. L. Wende11 (15)

Technical Information - Library (5)

Publishing Coordination (2)

\section{ONSITE}

2 DOE Richland Operations Office

P.0. Box 550

Richland, WA 99352

H. E. Ransom

R. K. Stewart

47 Pacific Northwest Laboratory

Battelle Boulevard

Richland, WA 99352

W. R. Barchet

J. W. Buck

J. R. Connel1

J. C. Doran

C. E. Elderkin

D. L. Elliott

R. L. George

D. L. Hadley

T. R. Hiester

A. H. Miller

E. L. Owzarski

W. T. Pennell

D. C. Powell 